\title{
Exciting inhibition: \\ Local regulation of synapse formation and plasticity
}


The studies described in this thesis were performed at the division of Cell Biology at the Faculty of Science of Utrecht University, The Netherlands.

This work is part of the research programme of the Foundation for Fundamental Research on Matter (FOM), which is part of the Dutch Research Council (NWO).

Cover and layout: Dennis L. H. Kruijssen.

Printed by Gildeprint.

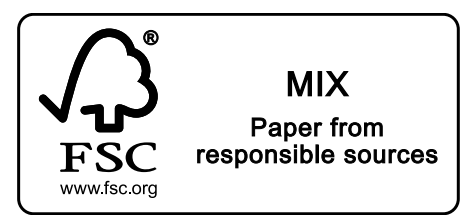

Copyright (c) Dennis L. H. Kruijssen, 2020.

All rights reserved. 


\title{
Exciting inhibition: \\ Local regulation of synapse formation and plasticity
}

Excitatie en inhibitie: lokale regulatie van synapsformatie en plasticiteit

(met een samenvatting in het Nederlands)

\begin{abstract}
Proefschrift
ter verkrijging van de graad van doctor aan de Universiteit Utrecht op gezag van de rector magnificus, prof.dr. H.R.B.M. Kummeling, ingevolge het besluit van het college voor promoties in het openbaar te verdedigen op
\end{abstract}

woensdag 4 maart 2020 des middags te 2.30 uur

door

Dennis Leonardus Hendrik Kruijssen

geboren op I4 juli 1990

te Amsterdam 
Promotor: Prof. dr. C.C. Hoogenraad

Copromotor: Dr. C.J.Wierenga 
Voor mijn moeder die me alles heeft geleerd 


\section{Table of Contents}

$\begin{array}{lll}\text { Chapter I General introduction } & 9\end{array}$

Chapter 2 Single synapse LTP: a matter of context?

Chapter 3 Endocannabinoid signaling mediates local dendritic

coordination between excitatory and inhibitory synapses

Chapter 4 Endocannabinoid signaling in inhibitory synapse dynamics and excitatory synaptic plasticity

Chapter 5 General discussion

Addendum

Curriculum Vitae

List of publications

Lekensamenvatting

148

Lay Summary

152

Acknowledgments 


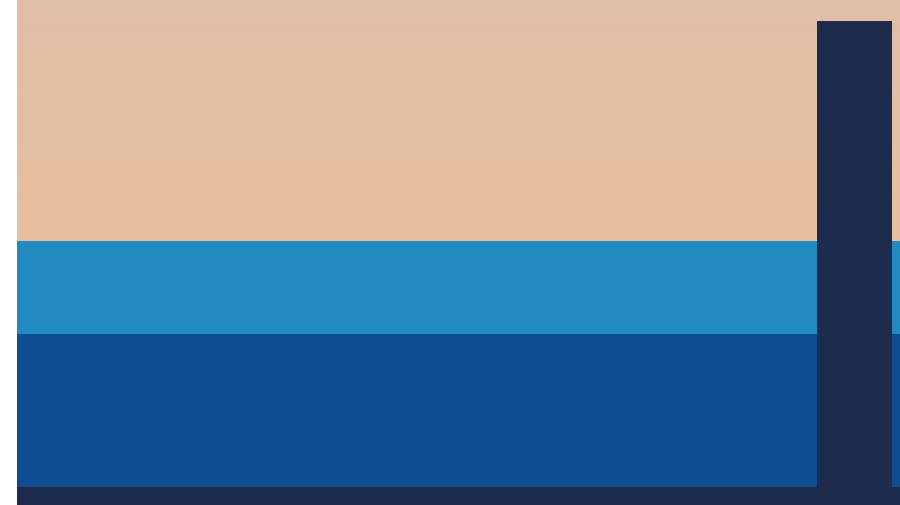




\title{
General introduction
}

\author{
Dennis L. H. Kruijssen
}


The brain is a fascinatingly complex organ. It enables us to process information from the outside world and produce an adequate response. The brain, however, is more than a black box that turns an input into a fixed output; it is capable of generating complex thoughts, ideas and emotions. But perhaps the most remarkable characteristic of the brain is that it is highly flexible: it is constantly changing and adapting to the signals it is processing. This allows us to retain and recall knowledge and to acquire skills; it allows us to learn. Our understanding of the brain is continuously improving, but there are still many mysteries left uncovered. One of the key questions in neuroscience: how are synaptic connections formed, and by what rules do they change? To start unraveling the answer to this question, we need to understand the workings of neurons and synapses - the fundamental building blocks of the brain.

\section{Neurons}

The human brain contains about $10^{11}$ neurons (Azevedo et al., 2009). Each of these neurons is connected to thousands of other neurons. Through these connections, neurons form complex networks. These networks use electrical signals to encode, process and send information. Several ion transporters maintain a high electrochemical gradient across the membrane, causing neurons to have a negative membrane potential in resting conditions. Changes in this membrane potential represent the signals that neurons use to communicate.

From the soma of the neuron, long processes originate that facilitate signal transmission to and from other neurons (Figure I). The axon is a long and thin process that originates from the soma or the proximal apical dendrite. Close to the beginning of the axon, the axon initial segment is located: a specialized area with an extremely high density of voltage-gated ion channels (Leterrier, 2018). When the membrane potential is sufficiently depolarized, these voltage-gated channels open and cause a rapid, transient and regenerative depolarization called the action potential that travels along the axon. The signal is transmitted to dendrites of other cells at synapses. At synapses, an action potential triggers the release of a chemical neurotransmitter that diffuses across the synaptic cleft to the dendrite, where it activates neurotransmitter receptors that translate the chemical signal into a small electrical signal called the postsynaptic potential. Dendrites are slightly thicker processes that are typically highly branched. The dendritic tree processes postsynaptic potentials and conducts them to the soma and the axon initial segment, where their sum could potentially lead to the initiation of an action potential.

Neurons can be categorized as excitatory or inhibitory, based on the neurotransmitter that is used in the axon. Excitatory neurotransmitters promote, while inhibitory neurotransmitters inhibit action potential firing in target neurons. In the cortex, excitatory neurons are typically pyramidal neurons, the name referring to the triangular shape of the soma. Inhibitory neurons are highly diverse. They can be categorized based on their morphology, action potential pattern, developmental lineage or expression of specific markers (Ascoli et al., 2008; Bloss et al., 20I6; Kepecs and Fishell, 20 I4; Lim et al., 20I8; Pelkey et al., 20I7;Wamsley and Fishell, 2017), but these classifications show (partial) overlap so no final consensus on inhibitory neuron types has been reached. Inhibitory neurons can also be categorized based on the area of the pyramidal neuron they target. Perisomatic-targeting inhibitory neurons make many synapses on the soma or axon initial segment. Dendritic-targeting inhibitory neurons come in many forms and make synapses onto different sections of the pyramidal neuron dendritic tree. This categorization is functionally relevant: perisomatic inhibitory synapses have a profound effect on action potential firing, while dendritic inhibitory synapses affect 
signal integration in the dendritic tree (see below) (Kubota, 20I4; Pelkey et al., 2017).

\section{Excitatory and inhibitory synapses}

\section{The presynapse}

The presynaptic side of both excitatory and inhibitory synapses is formed by specializations called boutons (Figure I). Neurotransmitter vesicles are accumulated in the bouton, together with the release machinery required to fuse these vesicles with the axonal membrane (Südhof,

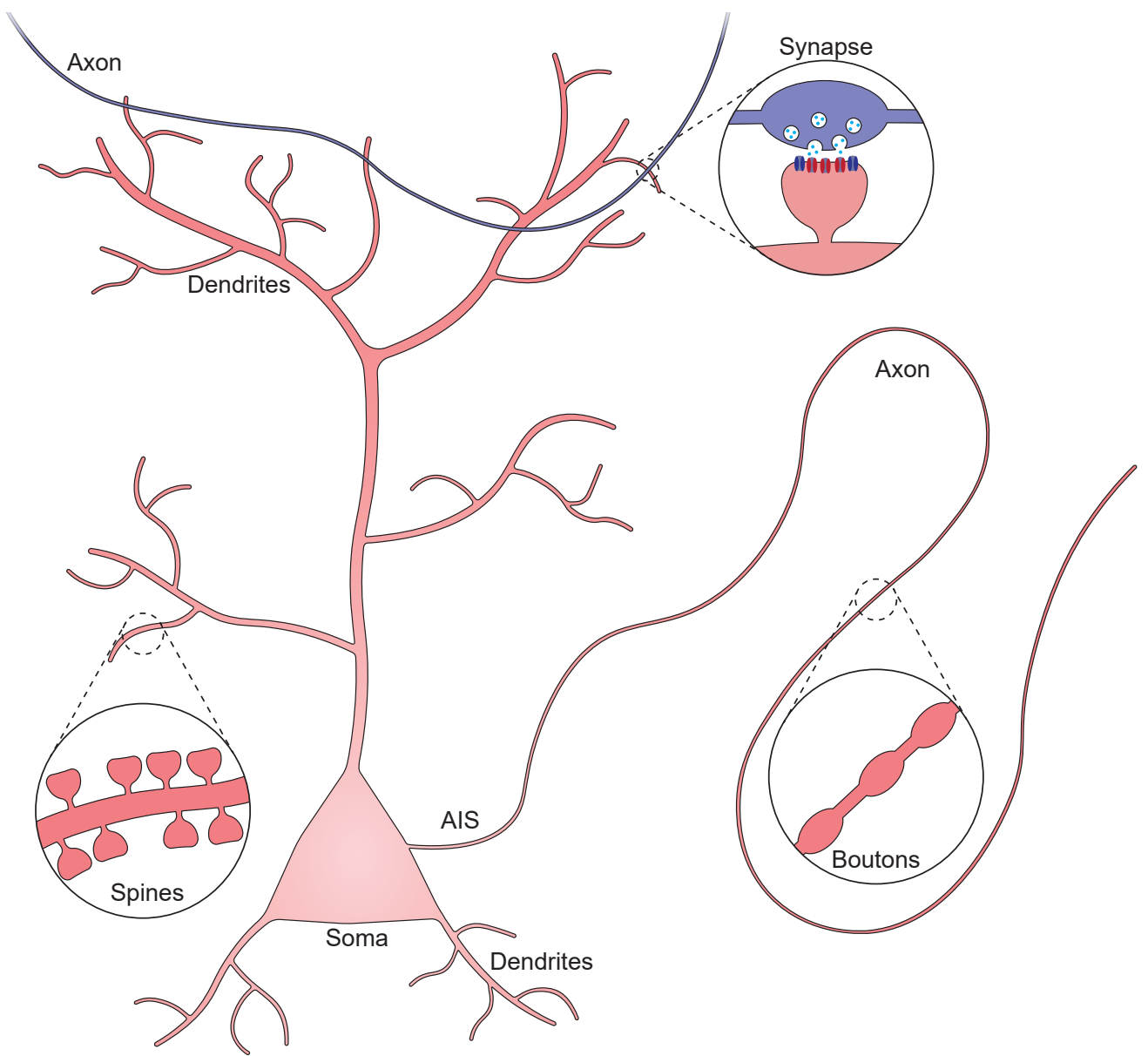

Figure I. Schematic representation of a typical pyramidal neuron.

A pyramidal neuron is schematically represented. The complex dendritic tree originates from the pyramid-shaped soma. When zooming in on the dendrites, one observes that it is covered in many protrusions called dendritic spines (left inset). The other process originating from the soma is the axon, and the initial part of the axon where action potentials are initiated is termed the axon initial segment (AIS). When zooming in on the axon, one observes swellings along the axon called boutons (bottom right inset). On the top, an axon from another neuron (blue) comes into view and makes contact with the dendrite of the red cell. Here, the bouton contacts the spine and together they form a synapse (top right inset). In the bouton, vesicles containing neurotransmitters (light blue) are located. When an action potential arrives at the bouton, these vesicles fuse with the membrane and release their contents into the synaptic cleft. At the membrane of the dendritic spine, the neurotransmitter (in this case glutamate) activates glutamate receptors (AMPA receptors, red, and NMDA receptors, dark blue). 
20I2). Vesicles in excitatory axons contain the main excitatory neurotransmitter glutamate, which is transported into the vesicle by the vesicular glutamate transporter (vGluT). In the case of inhibitory synapses, vesicles typically contain the main inhibitory neurotransmitter $\gamma$-aminobutyric acid (GABA), which is transported into the vesicles by the vesicular GABA transporter (VGAT). The vesicle fusion machinery is activated by an increase in calcium concentration and is triggered by action potentials through the activation of voltage-gated calcium channels (Südhof, 20I2). After vesicle fusion with the membrane, neurotransmitters are released in the synaptic cleft and can diffuse to the postsynapse.

\section{The excitatory postsynapse}

The excitatory postsynapse is typically situated on a protrusion of the dendritic membrane called the dendritic spine (Figure I). Glutamate receptors such as AMPA and NMDA receptors are found here, and these are kept in place by specialized postsynaptic density proteins, such as PSD-95, Homer and Shank (Scheefhals and MacGillavry, 2018; Sheng and Kim, 20 I I). Recently, it has been shown that there is some level of alignment between presynaptic release sites and postsynaptic glutamate receptors, which is thought to be regulated by transsynaptic adhesion molecules and likely increases the efficiency of synaptic transmission (Biederer et al., 20I7; Tang et al., 20I6).

When AMPA receptors are activated, the ion channel opens and allows for the flow of sodium and potassium (and in some cases calcium) ions. The net influx of positive ions through AMPA receptors is almost entirely responsible for the depolarization of the postsynaptic membrane. The ion channel of NMDA receptors is mainly permeable to calcium ions. The NMDA receptor is blocked by a magnesium ion under resting conditions and can only allow calcium inflow when receptor activation is paired with postsynaptic depolarization. NMDA receptors therefore act as coincidence detectors of presynaptic and postsynaptic activation. Recently, it has been suggested that NMDA receptors not only act as ion channels but can also act as metabotropic receptors, as some NMDA receptor-mediated effects can still be triggered after blocking the ion channel pore (Dore et al., 2016; Nabavi et al., 20I 3; Stein et al., 20I5). Finally, the excitatory postsynapse also contains group I metabotropic glutamate receptors. These $G_{q}$-protein coupled receptors activate several signaling pathways through activation of phospholipase $\mathrm{C}$, and can modulate neuronal excitability (Niswender and Conn, 2010).

\section{The inhibitory postsynapse}

Unlike the excitatory postsynapse, the inhibitory postsynapse has no morphological correlate. Inhibitory synapses typically directly contact the dendritic shaft (Megias et al., 200 I).About $20 \%$ of inhibitory synapses can form on dendritic spines, sharing this spine with an excitatory synapse (Bloss et al., 2016; Chen et al., 20I2; Villa et al., 20I6). GABA receptors are associated with the inhibitory postsynapse through the scaffolding protein and inhibitory synapse organizer gephyrin (Choii and Ko, 20I5; Tyagarajan and Fritschy, 2014). While the excitatory postsynapse is well understood, the proteins that compose and regulate the inhibitory postsynapse are only starting to be unraveled (Krueger-Burg et al., 2017; Sheng and Kim, 20I I; Uezu et al., 20I6).

The main inhibitory ionotropic receptor is the $\mathrm{GABA}_{\mathrm{A}}$ receptor. When activated by GABA, the chloride-permeable ion channel of the receptor opens. The negatively charged chloride ions flow into the neuron and usually cause a hyperpolarization of the membrane potential. 
GABA $_{A}$ receptors are enriched at inhibitory postsynapses but are also found extrasynaptically, where they mediate tonic inhibition (Farrant and Nusser, 2005; Sigel and Steinmann, 20I2). The $G_{A B A}$ receptor is a metabotropic G-protein coupled receptor. GABA $A_{B}$ receptors activate potassium channels that lead to a slow hyperpolarization of the neuron, but they can also mediate other processes in the pre- and postsynaptic neuron (Chalifoux and Carter, $20 \mathrm{II}$; Ulrich and Bettler, 2007).

\section{Dendritic integration}

Neurons receive many excitatory and inhibitory inputs: a typical rat CAI pyramidal neuron has $\sim 30000$ excitatory synapses and $\sim 1700$ inhibitory synapses throughout its dendritic tree (Megias et al., 200I). One of the major challenges of neuroscience is to understand how a neuron processes all these incoming signals and translates them into a useful output. The electrical signals generated by all these synapses are collected by the dendritic tree and propagate to the soma and the axon initial segment. There, an all-or-none decision is made: to fire or not to fire? For a long time, it was thought that this decision was the main level of information processing in the brain. It is now understood that the integration of signals that occurs in the dendrites actually forms another important layer of information processing. Individual dendritic branches are able to perform complex (nonlinear) computations with the incoming signals, well before they arrive at the soma (Branco and Häusser, 20 I0; London and Häusser, 2005; Polsky et al., 2004; Stuart and Spruston, 20I5; Ujfalussy et al., 20I 5).To unravel the neuronal code, we therefore need to understand the rules of dendritic integration.

The passive properties of dendrites (capacitance, resistance) heavily affect how synaptic potentials reach the soma. Dendrites act as poorly insulated electrical cables: as signals propagate along the dendrite, they are attenuated. A postsynaptic potential experiences a decrease in amplitude and an increase in width as it propagates towards the soma. When the most distal inputs arrive at the soma, they can potentially be completely abolished. Passive dendritic properties are not the same throughout the dendritic tree, but depend on dendritic morphology such as branch diameter and proximity to the tips of dendrites or to branching points (Gulledge et al., 2005; Rall, 1959, 1962; Spruston, 2009). Dendritic impedance is typically higher in tips and lower close to branching points. As synaptic depolarization is more efficient at high dendritic impedance, the location of the synapse along the proximaldistal axis can also affect the amplitude of the synaptic potential (Branco and Häusser, 20I I; Gulledge et al., 2005; Nevian et al., 2007; Rall and Rinzel, 1973; Spruston, 2009).When multiple synaptic potentials are induced close to each other, passive dendritic properties affect how these signals add up. The change in membrane potential induced by one synapse can reduce the driving force of a neighboring synapse. This results in sublinear summation of postsynaptic potentials (Figure 2). The summed postsynaptic potential can even saturate at large numbers of active synapses (London and Häusser, 2005; Tran-Van-Minh et al., 20I5). It has been shown that dendrites of some inhibitory neurons act as passive electrical cables and exhibit sublinear summation of inputs (Abrahamsson et al., 20I2; Hu and Vervaeke, 20 I8; Vervaeke et al., 2012).

It is however important to consider that most dendrites are not just passive electrical cables: they contain several types of voltage-gated ion channels that heavily influence dendritic integration (Johnston and Narayanan, 2008; Stuart and Spruston, 20 I5; Tran-Van-Minh et al., 2015). CAI pyramidal cells express NMDA channels as well as voltage-gated sodium and calcium channels that open when synaptic activity leads to sufficient depolarization, and 
A

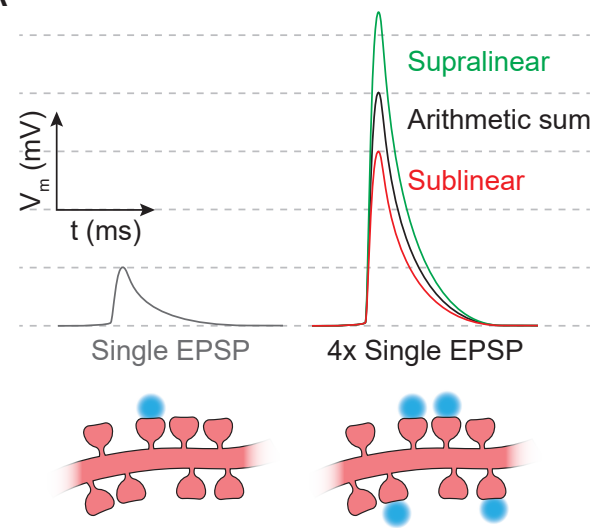

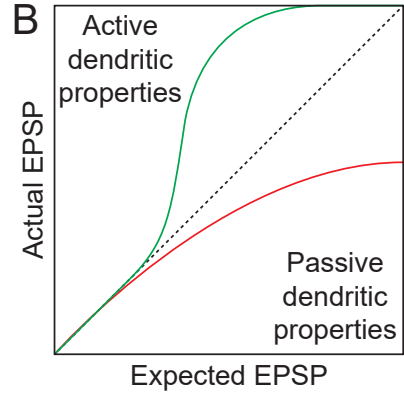

Figure 2. Nonlinear integration of synaptic inputs

A: Activation of a single excitatory synapse leads to an excitatory postsynaptic potential (EPSP, gray) with a certain amplitude. When simultaneously activating four synapses (with identical synaptic strength) on the same dendritic branch, one might expect that these EPSPs sum linearly, resulting in a large EPSP with four times the amplitude of the single synapse EPSP ("arithmetic sum", black). However, dendritic nonlinearities can cause these EPSPs to sum with a amplitude lower than the arithmetic sum ("sublinear", red) or higher than the arithmetic sum ("supralinear", green).

B: Schematic graph of the expected EPSP based on the arithmetic sum of individual inputs, versus the actual EPSP when measured. The dashed black line indicates the situation where synaptic inputs integrate linearly. Sublinear integration (red) is largely governed by passive dendritic properties and the collective EPSP amplitude can even saturate at large numbers of synapses. Due to active dendritic properties (i.e. the activation of voltage-gated ion channels), inputs can integrate supralinearly (green). As the activation of voltage-gated channels occurs after reaching a certain threshold, active dendrites often sum linearly at low numbers of synapses, but supralinearity quickly becomes apparent at larger numbers of synapses.

subsequently increase the magnitude of the depolarization. In some cases, these voltagegated channels are even involved in the generation of regenerative and propagating events called dendritic spikes. (Branco and Häusser, 20II; London and Häusser, 2005; Losonczy et al., 2008; Makara and Magee, 20I3; Nevian et al., 2007; Stuart and Spruston, 20I5;Tran-VanMinh et al., 2015). Through these mechanisms, synapses work together to produce a bigger response than the sum of their individual postsynaptic potentials (Figure 2).

The spatiotemporal pattern of synaptic activity in the dendritic tree crucially affects how signals are integrated. Due to the attenuation of signals as they travel along the dendritic tree, supralinear integration is most efficient when synaptic inputs are activated close to each other in space and time (Losonczy and Magee, 2006; Makara and Magee, 20I3). The gradient of input impedance along a dendritic branch makes it easier for distal inputs to recruit dendritic nonlinearities, while proximal inputs tend to integrate linearly (Branco and Häusser, 20I I). This effect also causes multiple synapses on the same dendritic branch to be integrated more efficiently when activated in distal-to-proximal order. (Branco et al., 20I0). When multiple synaptic inputs are separated in space, for example by being on separate dendritic branches, the attenuation of signals along the dendritic tree causes these inputs to sum linearly (Tran-Van-Minh et al., 2015).

Nonlinear integration is thus most efficient when synapses in close proximity are activated within a short time frame. Such an event might therefore seem unlikely if synapses would be distributed across the dendritic tree randomly. This is however not the case: both inhibitory 
and excitatory synapses are distributed in a structured, non-random way over dendritic branches (Bloss et al., 2016, 2018; Druckmann et al., 2014). Functional clustering has also been demonstrated: neighboring dendritic spines are more likely to be active at the same time (Kleindienst et al., 20I I) and in visual cortex, spines responding to similar visual stimuli tend to cluster together (lacaruso et al., 20I7; Wilson et al., 2016). Due to structural and functional clustering, nonlinear integration is likely to occur frequently in vivo. Indeed, it has been suggested that clustering and nonlinear dendritic integration play an important role in memory storage and recall (Kaifosh and Losonczy, 20I6; Kastellakis et al., 20I5).

\section{The role of inhibition in dendritic integration}

Dendritic inhibitory and excitatory synapses interact during dendritic integration. An inhibitory synapse can produce a hyperpolarization of the membrane, thereby reducing or even abolishing excitatory postsynaptic potentials that are nearby in space and time. However, often the local chloride reversal potential is similar to the membrane potential, such that an active inhibitory synapse does not result in a hyperpolarization. Despite this, these inhibitory synapses still affect excitatory signals through shunting inhibition: when an excitatory postsynaptic potential (EPSP) passes the open GABA $_{A}$ receptor channels, this temporarily results in some driving force, chloride ions flow through the open channels into the dendrite, and the EPSP is inhibited. (Spruston, 2009).

The location of an inhibitory synapse crucially affects its role in signal integration. Some interneuron types form synapses onto the soma or axon initial segment (perisomatic targeting) and these synapses directly interfere with action potential initiation by hyperpolarizing the membrane potential, making it harder for the cell to reach the action potential threshold. On the other hand, dendritically targeted inhibitory synapses affect signal integration in more subtle ways. Due to the strong attenuation of signals in the dendritic tree, inhibitory synapses on the dendrite most effectively interfere with excitatory inputs located on the same dendritic branch (Boivin and Nedivi, 20 I8; Stokes et al., 20 I4). Roughly 20\% of dendritic inhibitory synapses are located next to an excitatory synapse onto a dendritic spine (Bloss et al., 2016; Chen et al., 2012). Due to the high level of electrical compartmentalization of a dendritic spine, it is thought that such an inhibitory synapse can specifically inhibit that single excitatory input (Boivin and Nedivi, 2018; Chiu et al., 2013).

The precise location of the inhibitory synapse with respect to active excitatory synapses is important as well. An inhibitory synapse that is located on the path of an excitatory input towards the soma inhibits the propagation of the excitatory input to the soma more efficiently than an off-path inhibitory synapse (Hao et al., 2009). On the other hand, off-path inhibitory synapses close to the tip of the dendrite interfere with dendritic spike generation more efficiently (Gidon and Segev, 20I2). Indeed, inhibitory synapses also exert local control on the initiation of dendritic nonlinearities. A well-placed inhibitory synapse can efficiently prevent the activation of NMDA receptors (Schulz et al., 2018) and can prevent or suppress a dendritic spike (Doron et al., 20 I7; Jadi et al., 20 I2; Lovett-Barron et al., 20 I2; Müller et al., 2012). Dendritic integration is thus shaped locally by well-placed inhibitory synapses, that act as "traffic controllers" regulating the flow of information. However, to interfere with the initiation of dendritic nonlinearities, proximity of inhibitory synapses to excitatory inputs is required - especially when these excitatory synapses cooperate in structural and functional clusters. 


\section{Coordination of excitation and inhibition}

It is dazzling to imagine how a single neuron receives and processes thousands of excitatory and inhibitory inputs. How does the neuron cope with this cacophony of incoming signals? One aspect that is thought to be important for a neuron to function, is that excitatory and inhibitory inputs are coordinated. Since excitatory activity promotes, and inhibitory activity inhibits action potential initiation, fine-tuning excitation and inhibition to the right levels ensures that the action potential frequency stays within the functional range, where it can encode information. This view is however too simplistic, as inhibition does not only directly affect action potential initiation, but can regulate dendritic integration in intricate ways. Insufficient inhibition or excessive excitation will therefore hamper proper signal integration in dendrites. Disturbances in the coordination of excitation and inhibition are thought to be involved in several neurodevelopmental disorders, such as epilepsy, schizophrenia and autism (Lee et al., 20 I 7; Nelson andValakh, 20 I5;Tatti et al., 20 I7;Wondolowski and Dickman, 20 I3).

The concept of coordination of excitation and inhibition is often termed excitatory/inhibitory balance. While "balance" is an intuitive concept that can be used to think about excitation and inhibition, it is poorly defined and the concept is used in several related contexts. The term "balance" incorrectly implies equal numbers or equal collective strengths of excitatory and inhibitory synapses. Instead, it typically refers to some degree of coordination between excitation and inhibition, such that each are at a sufficient (but not equal) level to maintain neuronal function at all times.

Functionally, E/l coordination can be thought of in terms of the strength and timing of excitatory and inhibitory inputs. This can be challenging to study, as electrophysiology techniques do not allow isolation of excitatory and inhibitory inputs simultaneously. By alternatingly recording the excitatory and inhibitory response to an auditory stimulus, it has been shown that while early in development excitatory and inhibitory inputs are not coordinated, there is a high correlation between excitatory and inhibitory conductance in the auditory cortex of a more mature animal. Auditory stimulation can acutely improve the E/I correlation, suggesting that coordinated changes in synaptic strength are responsible for this maturation process (Dorrn et al., 20 I0). Similarly, in slices of visual cortex, it has been shown that the amplitude of evoked excitatory and inhibitory currents is different between cells but correlated within a cell. This coordination is maintained by somatically targeted inhibitory synapses whose synaptic strength is adapted based on the excitability of the pyramidal cell (Xue et al., 20l4). By recording from neighboring neurons with highly similar synaptic inputs, it has been shown that spontaneous activity or activity induced by sensory stimuli is strongly correlated in terms of strength and timing of excitatory and inhibitory inputs (although inhibition lags slightly behind excitation) (Okun and Lampl, 2008). Thus, excitatory and inhibitory conductances are often highly correlated in vivo. Computational modeling studies have suggested that such a tight balance increases the computational power of the neuron and that temporary imbalances regulate the gating of signal propagation through the neuronal network (Denève and Machens, 2016; Hennequin et al., 2017; Vogels and Abbott, 2009).

Secondly, one can look at E/l coordination in terms of the numbers of excitatory and inhibitory synapses. While inhibitory synapses are less prevalent than excitatory synapses, the ratio between the number of excitatory and inhibitory synapses seem to be preserved within dendritic branches in dissociated neurons (Liu, 2004). More recently it has been shown 
that in vivo, the densities of excitatory and inhibitory synapses differ across the branch types of the dendritic tree (Bloss et al., 2016; lascone et al., 2018). However, when looking at terminal dendritic branches, the $\mathrm{E} / \mathrm{l}$ ratio is preserved even within small dendritic segments (lascone et al., 2018). This suggests that regulatory mechanisms are in place to ensure such a small-scale coordination arises and is maintained. While it is not yet understood what these mechanisms are, they likely regulate the formation and plasticity of excitatory and inhibitory synapses, which are discussed in the following sections.

\section{Synapse formation}

\section{Excitatory synapse formation}

One potential mechanism to ensure coordination between excitation and inhibition arises and is maintained, is by fine-tuning synapse formation. Synapses are continuously being formed and removed. This is most prevalent during development when neuronal networks are being established, but persists into adulthood (Caroni et al., 2012; Holtmaat et al., 2005; Holtmaat and Svoboda, 2009). Excitatory synapse formation is thought to be mediated by dendritic protrusions called filopodia. These filopodia can contact axons or axonal filopodia (Fiala et al., 1998), thereby finding a presynaptic partner. Over time, these filopodia can either be removed, or can successfully develop into a functional and mature spine (Berry and Nedivi, 2017; Knott et al., 2006; Nägerl et al., 2007). Mature and persistent spines typically express the key postsynaptic protein PSD-95 (Cane et al., 20I4; Ehrlich et al., 2007; Villa et al., 20I6), and maturation of dendritic spines and recruitment of PSD-95 is promoted by sensory experience in visual cortex (Subramanian et al., 2019). In young neurons, the formation of individual excitatory synapses can be triggered by local exposure to glutamate (Kwon and Sabatini, 20II). This suggests that the presence of a functional glutamatergic presynapse nearby can stimulate the generation of a postsynapse, so that the synapse is completed. However, functional spines still form normally in absence of presynaptic glutamate release or without postsynaptic glutamate receptors (Lu et al., 20I3; Sigler et al., 20I7). Thus, the neuron likely intrinsically regulates the generation of dendritic spines throughout the dendritic tree, and this process can be fine-tuned by activity-dependent mechanisms.

The rate of spine formation and removal is dependent on brain area. In the hippocampus, spine turnover in vivo is so high that all spines can be replaced within 6 weeks (Attardo et al., 20I5), while turnover is significantly lower in cortex (Holtmaat et al., 2005; Yang et al., 2009). Additionally, changes in activity can crucially affect spine turnover. In vivo, deprivation of sensory input can cause a tremendous increase in the turnover of dendritic spines (Berry and Nedivi, 20I7; Hofer et al., 2009; Holtmaat et al., 2006; Keck et al., 2008), and these changes in connectivity are considered to be an adaptive mechanism in response to a change in presynaptic activity.

\section{Inhibitory synapse formation}

We do not yet fully understand what it takes to form, mature and maintain an inhibitory synapse, but important steps have been made. Inhibitory synapses typically form directly onto the dendritic shaft without the involvement of a dendritic protrusion (Wierenga et al., 2008). It is thought that inhibitory synapses are formed in a pre-before-post manner, as presynaptic marker VGAT typically appears before the postsynaptic marker gephyrin (Dobie and Craig, 20I I; Frias et al., 2019;Wierenga et al., 2008). Adhesion molecules such as 
neuroligin-2 and Sema4D likely play an important role in triggering and regulating synapse formation (Favuzzi and Rico, 2018; Frias and Wierenga, 20I3; Lu et al., 20I7). Work from our lab recently showed that Sema4D triggers the stabilization of inhibitory presynaptic boutons through PlexinBI-MET activation and downstream actin remodeling (Frias et al., 2019). Additionally, excreted factors such as BDNF, FGF7, and GABA itself play a role (Frias and Wierenga, 20I3). FGF7 is excreted from dendrites and specifically promotes inhibitory synapse maturation (Terauchi et al., 20I0). Furthermore, locally exposing the developing dendrite to GABA lead to the rapid formation of an inhibitory postsynapse (Oh et al., 20I6).

Inhibitory synapses can be highly dynamic: they appear, disappear and reappear on time scales ranging from tens of minutes to days (Chen et al., 2012; Dobie and Craig, 201 I; Frias et al., 20 I9; Schuemann et al., 20 I 3; Villa et al., 20 I6; Wierenga et al., 2008). The reappearance of synapses at the same location suggests that inhibitory synapse formation is not random but occurs at specific locations that are indicated by a yet unknown signal or marker (Frias et al., 2019; Villa et al., 2016; Wierenga et al., 2008). Inhibition can rapidly respond to activity perturbations (Chen et al., 20 I ; Keck et al., 20I I; Schuemann et al., 20 I3; van Versendaal et al., 20I2) and responds on shorter time scales than excitation (Keck et al., 20I I). Although we do not yet fully understand the role of this high level of dynamics, it is thought that rapid changes in inhibitory connectivity might represent a dynamic trial-and-error process to fine-tune inhibition at the right location (Wierenga, 2017) and allows quick adaptation to changing local needs for inhibition.

\section{Coordination of excitatory and inhibitory synapse formation}

As discussed above, the local ratio of excitatory and inhibitory synapses seem to be conserved. This suggests that the formation of synapses is somehow coordinated locally. We do not yet understand how such a coordination arises and is maintained. Some recent studies have presented proteins that regulate both excitatory and inhibitory synapse development, and might therefore play a role in the coordination of excitation and inhibition. The protein SRGAP2A is able to bind to both the excitatory postsynaptic density protein Homer and the inhibitory postsynaptic scaffold protein gephyrin. SRGAP2A promotes the maturation of excitatory and inhibitory synapses during development, while simultaneously reducing synaptic density (Fossati et al., 20I6). The protein CFYP and the adhesion protein cadherin- 10 are both enriched at excitatory and inhibitory synapses. These proteins are thought to be important for the coordination of excitatory and inhibitory synapses, as changing the level of these proteins reciprocally affects excitatory and inhibitory synapse numbers and strengths. (Davenport et al., 2019; Smith et al., 2017). Despite these interesting observations, important questions remain. While these studies show that some proteins coordinate the development of both excitatory and inhibitory synapses, we do not yet understand how the formation of inhibitory synapses is dependent on the level of excitation (or the other way around) in mature neurons. Furthermore, it is still unknown how excitatory and inhibitory synapse formation is coordinated on a local level.

\section{Excitatory and inhibitory synaptic plasticity}

\section{Excitatory synaptic plasticity}

A second potential mechanism to coordinate excitation and inhibition is by changes in synaptic strength. Synaptic plasticity - the ability of synapses to change their strength - occurs 
throughout the brain and is thought to be crucial for its capability to store information and to learn (Hayashi-Takagi et al., 20I5; Mayford et al., 20I2; Nicoll, 20I7; Takeuchi et al., 20I4). Long-term potentiation (LTP), arguably the best understood form of synaptic plasticity, involves a prolonged increase of synaptic transmission and can be induced by certain patterns of synaptic activity. Bliss and Lømo were the first to report in 1973 that repetitive stimulation of the perforant path in rabbit hippocampus lead to a significant increase in the amplitude of postsynaptic potentials that lasted for more than 30 minutes (Bliss and Lømo, 1973). Now, we know that high-frequency stimulation or theta burst stimulation patterns are able to robustly potentiate excitatory synapses. LTP is typically dependent on calcium entry through postsynaptic NMDA receptors and subsequent activation of the calcium-sensitive protein CaMKII (Lisman et al., 2012; Nicoll, 20I7). The expression site of LTP has been debated for a long time (Lømo, 20I8; Nicoll, 2017). It has now been shown that the strengthening of synapses mostly occurs through an increase of postsynaptic AMPA receptors (Granger and Nicoll, 20I3; Huganir and Nicoll, 20I3), but changes in the presynapse can also play a role (Bliss and Collingridge, 20I3; Castillo, 20I2). On the level of the single synapse, we are starting to understand the detailed mechanisms behind LTP, and this is discussed in chapter 2 .

Long-term depression (or weakening) of excitatory synapses typically occurs after prolonged low-frequency stimulation. It is mediated through NMDA receptor activation and the phosphatase calcineurin and leads to a decrease in surface AMPA receptors (Citri and Malenka, 2008; Lüscher and Malenka, 20I2). It has been suggested recently that LTD might be regulated through non-ionotropic NMDA-receptor signaling (Dore et al., 20I7; Nabavi et al., 20I3; Stein et al., 20I5). Alternatively, LTD can be regulated presynaptically by suppression of neurotransmitter release through $\mathrm{G}_{\mathrm{i} / \mathrm{o}}$-coupled GPCRs (Atwood et al., 2014).

Voltage-gated channels in the dendritic tree are not only important for dendritic integration (see above), but also allow the backpropagation of action potentials. These backpropagating action potentials (bAPs) are involved in synaptic plasticity, as they interact with incoming synaptic potentials by providing the depolarization required for NMDA channel opening. Calcium influx through NMDA channels is an essential trigger for many plasticity mechanisms. Indeed, the interaction of bAPs and synaptic activity is central to spike timing-dependent plasticity (STDP). STDP is a process where the timing of synaptic activity and a bAP ('spike') determines if a synapse undergoes LTP or LTD. If the bAP repeatedly follows synaptic activation this leads to LTP, while if the bAP repeatedly precedes synaptic activation, this leads to LTD (Caporale and Dan, 2008; Feldman, 2012; Markram et al., 1997; Stuart and Spruston, 2015). This mechanism is in correspondence with the prediction by Donald Hebb, who suggested in 1949 that when a presynaptic neuron can contribute to the spiking of a postsynaptic neuron, this connection is likely to be strengthened ("fire together, wire together") (Hebb, 1949).

Inhibitory synapses are able to locally interfere with calcium signaling, the key signal for LTP (Boivin and Nedivi, 20I8; Higley, 20I4). For example, a bAP can locally be abolished by the well-timed activity of an inhibitory synapse (Müllner et al., 20I5), thereby interfering with NMDA receptor activation and plasticity initiation. Additionally, local activation of GABA receptors during an LTP-inducing protocol has been shown to trigger the morphological correlate of long-term depression (Hayama et al., 20I3). It is therefore thought that inhibitory synapses are not only important for regulating dendritic integration, but are also coordinators of excitatory plasticity (Bar-llan et al., 20 I3; Boivin and Nedivi, 2018). 


\section{Inhibitory synaptic plasticity}

Like excitatory synapses, inhibitory synapses can strengthen and weaken. Inhibitory LTP (iLTP) or inhibitory LTD (iLTD) often occur heterosynaptically: they are dependent on the activation of glutamatergic synapses nearby. This implies a strong interaction between excitatory and inhibitory plasticity. Inhibitory plasticity can be regulated through changes in the postsynapse or the presynapse. Postsynaptically, changes in $\mathrm{GABA}_{\mathrm{A}}$ receptor trafficking or phosphorylation occur (Castillo et al., 20II). In primary hippocampal cultures, it has been shown that the activation of NMDA receptors can lead to an increased surface expression of $\mathrm{GABA}_{\mathrm{A}}$ receptors and an increased amplitude of inhibitory currents. This effect is mediated by the calcium sensor CaMKII (Marsden et al., 2007), which accumulates at inhibitory postsynapses upon weak glutamatergic activation (Marsden et al., 20I0). CaMKII causes the immobilization of $\mathrm{GABA}_{\mathrm{A}}$ receptors at the inhibitory synapse by phosphorylating the $\beta 3$ subunit of the receptor and subsequent recruitment of gephyrin (Petrini et al., 20I4). In acute slices of prefrontal cortex, it was shown that pharmacological activation of NMDA receptors could specifically potentiate dendritic inhibitory synapses of somatostatin-positive interneurons. This is regulated postsynaptically through the $\beta 2$ subunit of the $G_{A B A}$ receptor, which is enriched at these dendritic inhibitory synapses (Chiu et al., 2018).

Presynaptic inhibitory plasticity is often regulated through modulation of neurotransmitter release in the presynapse. This is triggered by glutamatergic activity, and often involves communication from the postsynaptic neuron to the presynaptic neuron.This communication is mediated by retrograde messengers: molecules that are secreted by the soma or dendrite and travel to the axon to activate receptors. Many retrograde messengers have been described throughout the nervous system, acting at excitatory and inhibitory synapses (Castillo et al., 20II; Regehr et al., 2009). The growth factor BDNF can be secreted by the postsynaptic neuron upon depolarization and activates the TrkB receptor in the presynapse, resulting in an increase of release probability (Magby et al., 2006). The gas nitric oxide is synthesized by nitric oxide synthase in the soma of layer 5 pyramidal neurons and can specifically enhance inhibitory synaptic transmission (Lourenço et al., 20I4). Certain lipid-derived molecules can also act as retrograde messengers. The most famous example is endocannabinoid signaling. This class of lipid molecules is known to play an important role in presynaptic LTD and is further discussed in chapter 4.

\section{Plasticity and the coordination of excitation and inhibition}

As synaptic plasticity causes the strength of synapses to continuously change, it is expected that mechanisms are in place to continuously correct these disturbances in E/I coordination. A cellular increase of excitatory or inhibitory synaptic strength can lead to an increase or decrease of action potential activity, respectively. Neurons coordinate their own excitability by mechanisms that are collectively referred to as homeostatic plasticity (Turrigiano, 20I2). Homeostatic synaptic scaling is a process where the strength of all excitatory synapses in a neuron collectively increase or decrease over time (Turrigiano, 2008). Activity deprivation leads to an increase of excitatory synaptic strength (Turrigiano et al., 1998), while simultaneously decreasing inhibitory synaptic strength (Kilman et al., 2002). This mechanism prevents that synaptic potentiation or depression accumulates and thus keeps the firing rate of the neuron stable (Turrigiano, 2008).

On shorter time scales simultaneous plasticity of excitatory and inhibitory synapses could be utilized to coordinate adjustments in synaptic strength. In organotypic hippocampal 
slices, electrical stimulation leads to strengthening of excitatory synapses, in parallel with an increase in size and number of perisynaptic gephyrin clusters. This could be indicative of inhibitory synapse formation and plasticity. (Flores et al., 20I5). An electron microscopy study by Bourne and Harris showed that that theta burst stimulation, known to induce strengthening of excitatory synapses, resulted in an increase of synaptic surface area for both excitatory and inhibitory synapses the CAI area of the hippocampus (Bourne and Harris, 20I I). In layer 5 pyramidal neurons, inhibitory LTP occurs simultaneously with spiketiming dependent LTP or LTD of excitatory synapses, in such a way that E/I ratio is reduced (D'amour and Froemke, 20I5). This is in agreement with predictions from computational studies that suggest that tight coordination of excitation and inhibition can arise from coordinated STDP of excitatory and inhibitory synapses (Hiratani and Fukai, 2017; Vogels et al., 20I I).

These mechanisms, as well as the heterosynaptic inhibitory plasticity mechanisms discussed above, have the potential to coordinate excitation and inhibition. Interestingly, while some mechanisms seem to coordinate excitatory and inhibitory plasticity to balance excitatory and inhibitory synaptic strength (Bourne and Harris, 20I I; Chiu et al., 20I8; D'amour and Froemke, 2015; Flores et al., 2015), others seem to increase the difference (Marsden et al., 2007, 20I0; Petrini et al., 20I4). To understand E/l coordination, we therefore need to understand under what circumstances these processes are triggered, and what are the (local) mechanisms mediating them. 


\section{Scope of this thesis}

Proper neuronal function is absolutely essential for the brain to enable us to remember and learn. Signals are integrated and processed in the dendritic tree and to process information properly a detailed coordination between excitation and inhibition is required. The studies on the coordination of excitatory and inhibitory synapse formation and plasticity that have been performed thus far only assess and manipulate synapse formation and plasticity on a large scale. However, integration of synaptic inputs occurs not only the level of the whole cell, but occurs already within dendrites. Additionally, the placement of inhibitory synapses strongly influences how the synapse can interact with dendritic integration. While these local aspects seem to be important for neuronal function, how the formation and plasticity of excitatory and inhibitory synapses is coordinated locally remains largely unexplored. In this thesis, I try to contribute to the knowledge on the coordination of excitatory and inhibitory synapse formation and plasticity by studying these processes at the level of the dendrite and the individual synapse.

Throughout this thesis, I used the hippocampus as a model system. The hippocampus is responsible for memory formation and is also thought to be involved in spatial navigation (Knierim, 20I5; Lisman et al., 2017). Because of its role in memory formation, as well as its particularly structured connectivity, it's a popular model system in neuroscience. The trisynaptic circuit of the hippocampus is highly structured and runs largely perpendicular to the long axis of the hippocampus (Knierim, 20I5). This is a major practical benefit, as the circuit largely remains intact when the hippocampus is sliced for experimental use. To be able to acquire fluorescence images of axons, dendrites and synapses, I used two-photon microscopy. This allowed me to perform imaging deep into these thick brain slices with minimal photodamage. I used two-photon glutamate uncaging to release glutamate with high temporal and spatial precision. Finally, I used patch clamp electrophysiology to record electrical activity of synapses in individual neurons.

To understand the local effects of excitatory synaptic plasticity, we first need to understand how a single synapse can be potentiated. In chapter 2 of this thesis, I review the literature on the long-term potentiation of single synapses. I consider the induction methods, expression and signaling pathways of LTP. I also discuss how LTP in a single synapse can interact with processes in its dendritic vicinity. I argue that single synapse LTP needs to be considered in the proper context, as the choice of induction method and interactions with the dendritic vicinity can affect LTP expression and signaling.

The central question of this thesis is if and how excitation and inhibition are coordinated locally. In chapter 3, we stimulated a small cluster of excitatory synapses using two-photon glutamate uncaging and showed that this can promote inhibitory presynaptic bouton growth on an axon-dendrite crossing nearby, a process mediated by the endocannabinoid 2-AG.

I further dissect the effect of endocannabinoid signaling on inhibitory synapse dynamics, growth and formation in chapter 4. This collection of preliminary data is in agreement with the hypothesis that endocannabinoid signaling promotes the growth of inhibitory boutons on short time scales and induces new inhibitory synapses on longer time scales.Additionally, endocannabinoids may affect the plasticity of excitatory synapses as well.

I finish this thesis by discussing and interpreting our findings more thoroughly, as well as providing an outlook to potential future experiments, in chapter 5 . 
General introduction 


\section{References}

Abrahamsson, T., Cathala, L., Matsui, K., Shigemoto, R., and DiGregorio, D.A. (20I2). Thin Dendrites of Cerebellar Interneurons Confer Sublinear Synaptic Integration and a Gradient of ShortTerm Plasticity. Neuron 73, II59-II72. doi:10.1016/j.neuron.2012.01.027.

Ascoli, G. A., Alonso-Nanclares, L., Anderson, S. A., Barrionuevo, G., Benavides-Piccione, R., Burkhalter, A., et al. (2008). Petilla terminology: nomenclature of features of GABAergic interneurons of the cerebral cortex. Nat. Rev. Neurosci. 9, 557-568. doi:I0.1038/nrn2402.

Attardo, A., Fitzgerald, J. E., and Schnitzer, M. J. (20I5). Impermanence of dendritic spines in live adult CAI hippocampus. Nature 523, 592-596. doi:10.1038/nature 14467.

Atwood, B. K., Lovinger, D. M., and Mathur, B. N. (20I4). Presynaptic long-term depression mediated by $\mathrm{G}_{\mathrm{i} / 0}$-coupled receptors. Trends Neurosci. 37, 663-673. doi:10.1016/j.tins.2014.07.010.

Azevedo, F.A. C., Carvalho, L. R. B., Grinberg, L.T., Farfel, J. M., Ferretti, R. E. L., Leite, R. E. P., et al. (2009). Equal Numbers of Neuronal and Nonneuronal Cells Make the Human Brain an Isometrically Scaled-Up Primate Brain. J. Comp. Neurol. 5I3, 532-54I. doi: I0.1002/cne.21974.

Bar-llan, L., Gidon, A., and Segev, I. (20I3). The role of dendritic inhibition in shaping the plasticity of excitatory synapses. Front. Neural Circuits 6 I-I3. doi:I0.3389/fncir.20|2.00 I I8.

Berry, K. P., and Nedivi, E. (2017). Spine Dynamics: Are They All the Same? Neuron 96, 43-55. doi:I0.1016/j.neuron.2017.08.008.

Biederer, T., Kaeser, P. S., and Blanpied, T. A. (2017). Transcellular Nanoalignment of Synaptic Function. Neuron 96, 680-696. doi:10.1016/j. neuron.2017.10.006.

Bliss, T.V. P., and Collingridge, G. L. (20I3). Expression of NMDA receptor-dependent LTP in the hippocampus: bridging the divide. Mol. Brain 6, I-I4. doi:I0.I I86/I756-6606-6-5.

Bliss, T. V. P., and Lømo, T. (1973). Long-lasting potentiation of synaptic transmission in the dentate area of the anaesthetized rabbit following stimulation of the perforant path. J. Physiol. 232, 33|-356. doi: 10.1 I I3/jphysiol. 1973. sp0I0273.
Bloss, E. B., Cembrowski, M. S., Karsh, B., Colonell, J., Fetter, R. D., and Spruston, N. (20I6). Structured Dendritic Inhibition Supports Branch-Selective Integration in CAI Pyramidal Cells. Neuron 89, 1016-1030. doi:10.1016/j.neuron.2016.01.029.

Bloss, E. B., Cembrowski, M. S., Karsh, B., Colonell, J., Fetter, R. D., and Spruston, N. (2018). Single excitatory axons form clustered synapses onto CAI pyramidal cell dendrites. Nat. Neurosci. 21, 353-363. doi:I0.1038/s4I593-0I8-0084-6.

Boivin, J. R., and Nedivi, E. (20|8). Functional implications of inhibitory synapse placement on signal processing in pyramidal neuron dendrites. Curr. Opin. Neurobiol. 5I, 16-22. doi:10.1016/j. conb.2018.01.013.

Bourne, J. N., and Harris, K. M. (20I I). Coordination of Size and Number of Excitatory and Inhibitory Synapses Results in a Balanced Structural Plasticity Along Mature Hippocampal CAI Dendrites During LTP. Hippocampus 21, 354373. doi: 10.1002/hipo.20768.

Branco, T., Clark, B. A., and Häusser, M. (2010). Dendritic Discrimination of Temporal Input Sequences in Cortical Neurons. Science 329, |67|-|675. doi:|0.| |26/science. | |89664.

Branco, T., and Häusser, M. (20I0). The single dendritic branch as a fundamental functional unit in the nervous system. Curr. Opin. Neurobiol. 20, 494502. doi:I0.1016/j.conb.2010.07.009.

Branco,T., and Häusser, M. (20I I). Synaptic Integration Gradients in Single Cortical Pyramidal Cell Dendrites. Neuron 69, 885-892. doi:10.1016/j. neuron.20II.02.006

Cane, M., Maco, B., Knott, G., and Holtmaat, A. (20|4). The Relationship between PSD-95 Clustering and Spine Stability In Vivo. J. Neurosci. 34, 20752086. doi:I0.I523/JNEUROSCI.3353-13.20I4

Caporale, N., and Dan, Y. (2008). Spike TimingDependent Plasticity: A Hebbian Learning Rule. Annu. Rev. Neurosci. 31, 25-46. doi:10.1/46/ annurev.neuro.31.060407.125639.

Caroni, P., Donato, F., and Muller, D. (20I2). Structural plasticity upon learning: Regulation and functions. Nat. Rev. Neurosci. 13, 478-490. doi:10.1038/ nrn3258.

Castillo, P. E. (20I2). Presynaptic LTP and LTD of Excitatory and Inhibitory Synapses. Cold Spring Harb. Perspect. Biol. 4, I-23. doi:I0.1I0I/ cshperspect.a005728. 
Castillo, P. E., Chiu, C. Q., and Carroll, R. C. (20II). Long-term plasticity at inhibitory synapses. Curr. Opin. Neurobiol. 21, 328-338. doi:10.1016/j. conb.2011.01.006.

Chalifoux, J. R., and Carter, A. G. (20II). GABA receptor modulation of synaptic function. Curr. Opin. Neurobiol. 21, 339-344. doi:10.1016/j. conb.20II.02.004.

Chen, J. L., Lin, W. C., Cha, J.W., So, P.T., Kubota, Y., and Nedivi, E. (20I I). Structural basis for the role of inhibition in facilitating adult brain plasticity. Nat. Neurosci. 14, 587-596. doi: 10.1038/nn.2799.

Chen, J. L., Villa, K. L., Cha, J. W., So, P. T. C., Kubota, Y., and Nedivi, E. (20I2). Clustered Dynamics of Inhibitory Synapses and Dendritic Spines in the Adult Neocortex. Neuron 74, 36I-373. doi:10.1016/j.neuron.2012.02.030.

Chiu, C. Q., Lur, G., Morse, T. M., Carnevale, N. T., Ellis-Davies, G. C. R., and Higley, M. J. (20I3). Compartmentalization of GABAergic Inhibition by Dendritic Spines. Science 340, 759-762. doi:10.1 I26/science. 1234274 .

Chiu, C. Q., Martenson, J. S., Yamazaki, M., Natsume, R., Sakimura, K., Tomita, S., et al. (20I8). InputSpecific NMDAR-Dependent Potentiation of Dendritic GABAergic Inhibition. Neuron 97, 368-377. doi:10.1016/j.neuron.2017.12.032.

Choii, G., and Ko, J. (20I5). Gephyrin: a central GABAergic synapse organizer. Exp. Mol. Med. 47, I-I0. doi:I0.1038/emm.20I5.5.

Citri, A., and Malenka, R. C. (2008). Synaptic Plasticity: Multiple Forms, Functions, and Mechanisms. Neuropsychopharmacology 33, 18-41. doi:10.1038/sj.npp. I301559.

D'amour,J.A., and Froemke, R. C. (20I5). Inhibitory and Excitatory Spike-Timing-Dependent Plasticity in the Auditory Cortex. Neuron 86, 5I4-528. doi:I0.1016/j.neuron.2015.03.014.

Davenport, E. C., Szulc, B. R., Drew, J., Taylor, J., Morgan, T., Higgs, N. F., et al. (2019). Autism and Schizophrenia-Associated CYFIPI Regulates the Balance of Synaptic Excitation and Inhibition. Cell Rep. 26, 2037-205I. doi:10.1016/j. celrep.2019.01.092.

Denève, S., and Machens, C. K. (20I6). Efficient codes and balanced networks. Nat. Neurosci. 19, 375382. doi: 10.1038/nn.4243.

Dobie, F. A., and Craig, A. M. (20II). Inhibitory
Synapse Dynamics: Coordinated Presynaptic and Postsynaptic Mobility and the Major Contribution of Recycled Vesicles to New Synapse Formation. J. Neurosci. 31, 1048I-10493. doi:10.1523/ jneurosci.6023-10.2011.

Dore, K., Aow, J., and Malinow, R. (2016). The Emergence of NMDA Receptor Metabotropic Function: Insights from Imaging. Front. Synaptic Neurosci. 8, I-9. doi:10.3389/fnsyn.2016.00020.

Dore, K., Stein, I. S., Brock, J. A., Castillo, P. E., Zito, K., and Sjöström, P.J. (2017). Unconventional NMDA Receptor Signaling. J. Neurosci. 37, 10800-10807. doi:I0.1523/JNEUROSCI.1825-17.20I7.

Doron, M., Chindemi, G., Muller, E., Markram, H., and Segev, I. (2017). Timed Synaptic Inhibition Shapes NMDA Spikes, Influencing Local Dendritic Processing and Global I/O Properties of Cortical Neurons. Cell Rep. 21, 1550-1561. doi:10.1016/j. celrep.2017.10.035.

Dorrn, A. L., Yuan, K., Barker, A. J., Schreiner, C. E., and Froemke, R. C. (20I0). Developmental sensory experience balances cortical excitation and inhibition. Nature 465, 932-936. doi:10.1038/ nature09II9.

Druckmann, S., Feng, L., Lee, B., Yook, C., Zhao, T., Magee, J. C., et al. (20|4). Structured Synaptic Connectivity between Hippocampal Regions. Neuron 8I, 629-640. doi:10.1016/j. neuron.2013.11.026.

Ehrlich, I., Klein, M., Rumpel, S., and Malinow, R. (2007). PSD-95 is required for activity-driven synapse stabilization. Proc. Natl. Acad. Sci. 104, 4I764I8I. doi: 10.1073/pnas.0609307I04.

Farrant, M., and Nusser, Z. (2005). Variations on an inhibitory theme: Phasic and tonic activation of GABA A receptors. Nat. Rev. Neurosci. 6, 215229. doi: $10.1038 / \mathrm{nrn} 1625$.

Favuzzi, E., and Rico, B. (20I8). Molecular diversity underlying cortical excitatory and inhibitory synapse development. Curr. Opin. Neurobiol. 53, 8-I5. doi:10.1016/j.conb.2018.03.0II.

Feldman, D. E. (20I2). The Spike-Timing Dependence of Plasticity. Neuron 75, 556-57I. doi:10.1016/j. neuron.20I2.08.00I

Fiala, J. C., Feinberg, M., Popov, V., and Harris, K. M. (1998). Synaptogenesis Via Dendritic Filopodia in Developing Hippocampal Area CAI. J. Neurosci. 18, 8900-891I. doi:10.1523/ JNEUROSCI.18-21-08900.1998. 
Flores, C. E., Nikonenko, I., Mendez, P., Fritschy, J.-M., Tyagarajan, S. K., and Muller, D. (20I5). Activitydependent inhibitory synapse remodeling through gephyrin phosphorylation. Proc. Natl. Acad. Sci. II2, E65-72. doi:10.1073/ pnas. I4III70II 2.

Fossati, M., Pizzarelli, R., Schmidt, E. R., Kupferman, J. V, Stroebel, D., Polleux, F., et al. (20I6). SRGAP2 and Its Human-Specific Paralog Co-Regulate the Development of Excitatory and Inhibitory Synapses. Neuron 91, 356-369. doi:10.1016/j. neuron.2016.06.013.

Frias, C. P., Liang, J., Bresser, T., Scheefhals, L., van Kesteren, M., van Dorland, R., et al. (2019). Semaphorin4D Induces Inhibitory Synapse Formation by Rapid Stabilization of Presynaptic Boutons via MET Coactivation. J. Neurosci. 39, 422 I-4237. doi:I0.1523/jneurosci.0215-19.2019.

Frias, C. P., and Wierenga, C. J. (20/3). Activitydependent adaptations in inhibitory axons. Front. Cell. Neurosci. 7, I-16. doi:10.3389/ fncel.2013.00219.

Gidon, A., and Segev, I. (20I2). Principles Governing the Operation of Synaptic Inhibition in Dendrites. Neuron 75, 330-34I. doi:10.1016/j. neuron.20I2.05.015

Granger, A. J., and Nicoll, R. A. (2013). Expression mechanisms underlying long-term potentiation: a postsynaptic view, 10 years on. Philos. Trans. R. Soc. B Biol. Sci. 369, I-6. doi:10.1098/ rstb.2013.0136.

Gulledge, A. T., Kampa, B. M., and Stuart, G. J. (2005) Synaptic Integration in Dendritic Trees. J. Neurobiol. 64, 75-90. doi:I0.I002/neu.20I44.

Hao, J.,Wang, X., Dan, Y., Poo, M., and Zhang, X. (2009). An arithmetic rule for spatial summation of excitatory and inhibitory inputs in pyramidal neurons. Proc. Natl.Acad. Sci. 106, 21906-21911. doi:I0.1073/pnas.0912022106.

Hayama, T., Noguchi, J., Watanabe, S., Takahashi, N., Hayashi-Takagi, A., Ellis-Davies, G. C. R., et al. (20I3). GABA promotes the competitive selection of dendritic spines by controlling local $\mathrm{Ca}^{2+}$ signaling. Nat. Neurosci. 16, 1409-1416. doi:10.1038/nn.3496.

Hayashi-Takagi, A., Yagishita, S., Nakamura, M., Shirai, F., Wu, Y. I., Loshbaugh, A. L., et al. (20I5). Labelling and optical erasure of synaptic memory traces in the motor cortex. Nature 525, 333-338. doi:10.1038/nature 15257
Hebb, D. O. (1949). The organization of behavior; a neuropsychological theory. Oxford, England: Wiley.

Hennequin, G., Agnes, E. J., and Vogels, T. P. (2017). Inhibitory Plasticity: Balance, Control, and Codependence. Annu. Rev. Neurosci. 40, 557579. doi: I0. I | 46/annurev-neuro-072 I |6-03 I005.

Higley, M. J. (20I4). Localized GABAergic inhibition of dendritic $\mathrm{Ca}^{2+}$ signalling. Nat. Rev. Neurosci. I5, 567-572. doi: 10.1038/nrn3803.

Hiratani, N., and Fukai, T. (20I7). Detailed Dendritic Excitatory/Inhibitory Balance through Heterosynaptic Spike-Timing-Dependent Plasticity. J. Neurosci. 37, 12106-12122. doi:I0.I523/JNEUROSCI.0027-I7.20I7.

Hofer, S. B., Mrsic-Flogel, T. D., Bonhoeffer, T., and Hübener, M. (2009). Experience leaves a lasting structural trace in cortical circuits. Nature 457 , 3|3-3|7. doi:10.1038/nature07487.

Holtmaat, A. J. G. D., Trachtenberg, J. T., Wilbrecht, L., Shepherd, G. M., Zhang, X., Knott, G. W., et al. (2005). Transient and persistent dendritic spines in the neocortex in vivo. Neuron 45, 279-291. doi:10.1016/j.neuron.2005.01.003.

Holtmaat, A., and Svoboda, K. (2009). Experiencedependent structural synaptic plasticity in the mammalian brain. Nat. Rev. Neurosci. 10, 647658. doi:10.1038/nrn2699.

Holtmaat,A.,Wilbrecht, L., Knott, G.W.,Welker, E., and Svoboda, K. (2006). Experience-dependent and cell-type-specific spine growth in the neocortex. Nature 44I, 979-983. doi:I0. 1038/nature04783.

$\mathrm{Hu}, \mathrm{H}$. , and Vervaeke, K. (20I8). Synaptic integration in cortical inhibitory neuron dendrites. Neuroscience 368, ||15-13|. doi:10.1016/j. neuroscience.2017.06.065.

Huganir, R. L., and Nicoll, R. A. (20I3). AMPARs and Synaptic Plasticity:The Last 25 Years. Neuron 80 , 704-7|7. doi:10.1016/j.neuron.2013.10.025.

lacaruso, M. F., Gasler, I. T., and Hofer, S. B. (20I7). Synaptic organization of visual space in primary visual cortex. Nature 547, 449-452. doi:10.1038/ nature23019.

lascone, D. M., Li, Y., Sümbül, U., Doron, M., Chen, H., Sumbul, U., et al. (20/8). Whole-neuron synaptic mapping reveals local balance between excitatory and inhibitory synapse organization. bioRxiv, I-42. doi: I0.I I0I/395384. 
Jadi, M., Polsky, A., Schiller, J., and Mel, B. W. (20I2) Location-dependent effects of inhibition on local spiking in pyramidal neuron dendrites. PLoS Comput. Biol. 8. doi:I0.137//journal. pcbi. 1002550.

Johnston, D., and Narayanan, R. (2008). Active dendrites: colorful wings of the mysterious butterflies. Trends Neurosci. 31, 309-316. doi:10.1016/j.tins.2008.03.004.

Kaifosh, P., and Losonczy, A. (2016). Mnemonic Functions for Nonlinear Dendritic Integration in Hippocampal Pyramidal Circuits. Neuron 90, 622-634. doi:10.1016/j.neuron.2016.03.019.

Kastellakis, G., Cai, D. J., Mednick, S. C., Silva, A. J., and Poirazi, P. (20I5). Synaptic clustering within dendrites: An emerging theory of memory formation. Prog. Neurobiol. 126, 19-35. doi:I0.1016/j.pneurobio.2014.12.002.

Keck, T., Mrsic-Flogel, T. D., Vaz Afonso, M., Eysel, U. T., Bonhoeffer, T., and Hübener, M. (2008). Massive restructuring of neuronal circuits during functional reorganization of adult visual cortex. Nat. Neurosci. II, II62-II67. doi:10.1038/ nn.2I8I.

Keck, T., Scheuss, V., Jacobsen, R. I., Wierenga, C. J., Eysel, U. T., Bonhoeffer, T., et al. (20II). Loss of Sensory Input Causes Rapid Structural Changes of Inhibitory Neurons in Adult Mouse Visual Cortex. Neuron 7I, 869-882. doi:10.1016/j. neuron.20II.06.034.

Kepecs, A., and Fishell, G. (20I4). Interneuron cell types are fit to function. Nature 505, 318-326. doi: I0.1038/nature 12983.

Kilman, V., van Rossum, M. C.W., and Turrigiano, G. G. (2002). Activity Deprivation Reduces Miniature IPSC Amplitude by Decreasing the Number of

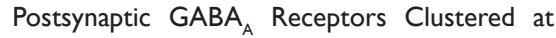
Neocortical Synapses. J. Neurosci. 22, 1328|337. doi: | 0.1523/jneurosci.22-04-0| 328.2002.

Kleindienst, T., Winnubst, J., Roth-Alpermann, C., Bonhoeffer, T., and Lohmann, C. (20II). Activity-Dependent Clustering of Functional Synaptic Inputs on Developing Hippocampal Dendrites. Neuron 72, 1012-1024. doi:10.1016/j. neuron.2011.10.015.

Knierim, J. J. (20I5). The hippocampus. Curr. Biol. 25, RIII6-RII2I. doi:I0.I0I6/j.cub.20I5.10.049.

Knott, G. W., Holtmaat, A., Wilbrecht, L., Welker, E., and Svoboda, K. (2006). Spine growth precedes synapse formation in the adult neocortex in vivo. Nat. Neurosci. 9, I I 17-I I24. doi: 10.1038/ nnI747.

Krueger-Burg, D., Papadopoulos, T., and Brose, N. (2017). Organizers of inhibitory synapses come of age. Curr. Opin. Neurobiol. 45, 66-77. doi:I0.1016/j.conb.2017.04.003.

Kubota,Y. (20I4). Untangling GABAergic wiring in the cortical microcircuit. Curr. Opin. Neurobiol. 26, 7-I4. doi:10.1016/j.conb.2013.10.003.

Kwon, H.-B., and Sabatini, B. L. (20II). Glutamate induces de novo growth of functional spines in developing cortex. Nature 474, 100-104. doi:10.1038/nature09986.

Lee, E., Lee, J., and Kim, E. (20I7). Excitation/Inhibition Imbalance in Animal Models of Autism Spectrum Disorders. Biol. Psychiatry 8I, 838-847. doi:I0.10I6/j.biopsych.2016.05.0II.

Leterrier, C. (2018). The Axon Initial Segment: An Updated Viewpoint. J. Neurosci. 38, 2135-2145. doi:10.1523/JNEUROSCI.1922-17.2018.

Lim, L., Mi, D., Llorca, A., and Marín, O. (20I8). Development and Functional Diversification of Cortical Interneurons. Neuron 100, 294-313. doi:10.1016/j.neuron.2018.10.009.

Lisman, J., Buzsáki, G., Eichenbaum, H., Nadel, L., Ranganath, C., and Redish, A. D. (2017). Viewpoints: how the hippocampus contributes to memory, navigation and cognition. Nat. Neurosci. 20, 1434-I447. doi: I0.1038/nn.466I.

Lisman, J., Yasuda, R., and Raghavachari, S. (20I2). Mechanisms of CaMKII action in long-term potentiation. Nat. Rev. Neurosci. 13, 169-182. doi:10.1038/nrn3192.

Liu, G. (2004). Local structural balance and functional interaction of excitatory and inhibitory synapses in hippocampal dendrites. Nat. Neurosci. 7, 373379. doi: I0.1038/nn I 206.

Lømo, T. (20I8). Discovering long-term potentiation (LTP) - recollections and reflections on what came after. Acta Physiol. 222, I-22. doi: I0.1 I I I/ apha. I292I.

London, M., and Häusser, M. (2005). Dendritic computation. Annu. Rev. Neurosci. 28, 503-532. doi:I0.I |46/annurev.neuro.28.06|604.I35703.

Losonczy, A., and Magee, J. C. (2006). Integrative 
Properties of Radial Oblique Dendrites in Hippocampal CAI Pyramidal Neurons. Neuron 50, 29I-307. doi:I0.1016/j.neuron.2006.03.016.

Losonczy, A., Makara, J. K., and Magee, J. C. (2008). Compartmentalized dendritic plasticity and input feature storage in neurons. Nature 452, 436-44I. doi: I0. I038/nature06725.

Lourenço, J., Pacioni, S., Rebola, N., van Woerden, G. M., Marinelli, S., DiGregorio, D., et al. (20I4). Nonassociative Potentiation of Perisomatic Inhibition Alters the Temporal Coding of Neocortical Layer 5 Pyramidal Neurons. PLoS Biol. 12, I-19. doi: 10.1371/journal.pbio.1001903.

Lovett-Barron, M., Turi, G. F., Kaifosh, P., Lee, P. H., Bolze, F., Sun, X.-H., et al. (20I2). Regulation of neuronal input transformations by tunable dendritic inhibition. Nat. Neurosci. 15, 423-430. doi: $10.1038 / \mathrm{nn} .3024$

Lu, W., Bromley-Coolidge, S., and Li, J. (2017). Regulation of GABAergic synapse development by postsynaptic membrane proteins. Brain Res. Bull. 129, 30-42. doi:10.1016/j. brainresbull.2016.07.004.

Lu, W., Bushong, E. A., Shih, T. P., Ellisman, M. H., and Nicoll, R. A. (20/3). The Cell-Autonomous Role of Excitatory Synaptic Transmission in the Regulation of Neuronal Structure and Function. Neuron 78, 433-439. doi:10.1016/j. neuron.2013.02.030.

Lüscher, C., and Malenka, R. C. (20I2). NMDA Receptor-Dependent Long-Term Potentiation and Long-Term Depression (LTP/LTD). Cold Spring Harb. Perspect. Biol. 4, I-16. doi:10.1 I0I/ cshperspect.a0057I0.

Magby, J. P., Bi, C., Chen, Z.-Y., Lee, F. S., and Plummer, M. R. (2006). Single-cell Characterization of Retrograde Signaling by Brain-Derived Neurotrophic Factor. J. Neurosci. 26, I3531I3536. doi:I0.1523/JNEUROSCI.4576-06.2006.

Makara,J. K., and Magee,J.C.(20I3).Variable Dendritic Integration in Hippocampal CA3 Pyramidal Neurons. Neuron 80, I438-1450. doi:10.1016/j. neuron.2013.10.033

Markram, H., Lübke, J., Frotscher, M., and Sakmann, B. (1997). Regulation of Synaptic Efficacy by Coincidence of Postsynaptic APs and EPSPs. Science 275, 213-215. doi:10.1126/ science. 275.5297 .213 .

Marsden, K. C., Beattie, J. B., Friedenthal, J., and
Carroll, R. C. (2007). NMDA Receptor Activation Potentiates Inhibitory Transmission through GABA Receptor-Associated ProteinDependent Exocytosis of $\mathrm{GABA}_{\mathrm{A}}$ Receptors. J. Neurosci. 27, 14326-14337. doi:10.1523/ JNEUROSCI.4433-07.2007.

Marsden, K. C., Shemesh, A., Bayer, K. U., and Carroll, R. C. (20I0). Selective translocation of $\mathrm{Ca}^{2+} /$ calmodulin protein kinase Il $\alpha$ (CaMKIl $\alpha)$ to inhibitory synapses. Proc. Natl. Acad. Sci. 107, 20559-20564. doi:I0.1073/pnas. I0I0346I 07.

Mayford, M., Siegelbaum, S.A., and Kandel, E. R. (20I2). Synapses and memory storage. Cold Spring Harb. Perspect. Biol. 4, I-18. doi:I0.1 I0I/cshperspect. a00575I.

Megías, M., Emri, Z., Freund,T. F., and Gulyás,A. I. (200I). Total number and distribution of inhibitory and excitatory synapses on hippocampal CAI pyramidal cells. Neuroscience 102, 527-540. doi: I0.1016/S0306-4522(00)00496-6.

Müller, C., Beck, H., Coulter, D., and Remy, S. (20I2). Inhibitory Control of Linear and Supralinear Dendritic Excitation in CAI Pyramidal Neurons. Neuron 75, 85I-864. doi:10.1016/j. neuron.2012.06.025.

Müllner, F. E.,Wierenga, C. J., and Bonhoeffer,T. (20I5). Precision of Inhibition: Dendritic Inhibition by Individual GABAergic Synapses on Hippocampal Pyramidal Cells Is Confined in Space and Time. Neuron 87, 576-589. doi:10.1016/j. neuron.2015.07.003.

Nabavi, S., Kessels, H. W., Alfonso, S., Aow, J., Fox, R., and Malinow, R. (20I3). Metabotropic NMDA receptor function is required for NMDA receptor-dependent long-term depression. Proc. Natl. Acad. Sci. II0, 4027-4032. doi:10.1073/ pnas. I 2 I9454IIO.

Nägerl, U. V., Köstinger, G., Anderson, J. C., Martin, K. A. C., and Bonhoeffer, T. (2007). Protracted Synaptogenesis after Activity-Dependent Spinogenesis in Hippocampal Neurons. J. Neurosci. 27, 8I49-8156. doi:10.1523/ JNEUROSCI.05। I-07.2007.

Nelson, S. B., and Valakh, V. (20I5). Excitatory/ Inhibitory Balance and Circuit Homeostasis in Autism Spectrum Disorders. Neuron 87, 684698. doi:10.1016/j.neuron.2015.07.033.

Nevian,T., Larkum, M. E., Polsky,A., and Schiller,J. (2007). Properties of basal dendrites of layer 5 pyramidal neurons: a direct patch-clamp recording study. 
Nat. Neurosci. 10, 206-2 |4. doi:| 0. I038/nn |826.

Nicoll, R. A. (2017). A Brief History of Long-Term Potentiation. Neuron 93, 28I-290. doi:10.1016/j. neuron.2016.12.015.

Niswender, C. M., and Conn, P.J. (2010). Metabotropic Glutamate Receptors: Physiology, Pharmacology and Disease. Annu. Rev. Pharmacol. Toxicol. 50, 295-322. doi:I0.I 146/annurev. pharmtox.01 1008.145533.

Oh, W. C., Lutzu, S., Castillo, P. E., and Kwon, H.-B. (2016). De novo synaptogenesis induced by GABA in the developing mouse cortex. Science 353, 1037-1040. doi:10.1 126/science.aaf5206.

Okun, M., and Lampl, I. (2008). Instantaneous correlation of excitation and inhibition during ongoing and sensory-evoked activities. Nat. Neurosci. II, 535-537. doi: 10.1038/nn.2105.

Pelkey, K. A., Chittajallu, R., Craig, M. T., Tricoire, L., Wester,J.C., and McBain,C.J.(2017). Hippocampal GABAergic inhibitory interneurons. Physiol. Rev. 97, 1619-1747. doi:10.1152/physrev.00007.2017.

Petrini, E. M., Ravasenga, T., Hausrat, T. J., lurilli, G., Olcese, U., Racine, V., et al. (20l4). Synaptic recruitment of gephyrin regulates surface $\mathrm{GABA}_{\mathrm{A}}$ receptor dynamics for the expression of inhibitory LTP. Nat. Commun. 5, I-19. doi:10.1038/ncomms492I.

Polsky, A., Mel, B. W., and Schiller, J. (2004). Computational subunits in thin dendrites of pyramidal cells. Nat. Neurosci. 7, 62I-627. doi: I0.1038/nnI253.

Rall, W. (1959). Branching Dendritic Trees and Motoneuron Membrane Resistivity. Exp. Neurol. I, 49|-527. doi: I0.1016/00|4-4886(59)90046-9.

Rall,W. (1962). Theory of Physiological Properties of Dendrites. Ann. N. Y. Acad. Sci. 96, I07I-1092. doi:I0.I I II/j.1749-6632.1962.tb54I20.x.

Rall,W., and Rinzel, J. (1973). Branch Input Resistance and Steady Attenuation for Input to One Branch of a Dendritic Neuron Model. Biophys. J. I3, 648688. doi: $10.1016 / 50006-3495(73) 86014-X$.

Regehr, W. G., Carey, M. R., and Best, A. R. (2009). Activity-Dependent Regulation of Synapses by Retrograde Messengers. Neuron 63, 154-170. doi:10.1016/j.neuron.2009.06.021.

Scheefhals, N., and MacGillavry, H.D. (20I8). Functional organization of postsynaptic glutamate receptors. Mol. Cell. Neurosci. 91, 82-94. doi:10.1016/j. mcn.20l8.05.002.

Schuemann, A., Klawiter, A., Bonhoeffer, T., and Wierenga, C. J. (20/3). Structural plasticity of GABAergic axons is regulated by network activity and $\mathrm{GABA}_{A}$ receptor activation. Front. Neural Circuits 7, II3. doi:10.3389/ fncir.2013.00113.

Schulz, J. M., Knoflach, F., Hernandez, M.-C., and Bischofberger, J. (2018). Dendrite-targeting interneurons control synaptic NMDA-receptor activation via nonlinear $\alpha 5-G A B A_{A}$ receptors. Nat. Commun. 9, I-I6. doi:I0.1038/s4|467-0I806004-8.

Sheng, M., and Kim, E. (20II). The Postsynaptic Organization of Synapses. Cold Spring Harb. Perspect. Biol. 3, I-20. doi:I0.1 I0I/cshperspect. a005678.

Sigel, E., and Steinmann, M. E. (20I2). Structure, function, and modulation of $\mathrm{GABA}_{\mathrm{A}}$ receptors. J. Biol. Chem. 287, 40224-4023I. doi: I0. 1074/jbc. RII 2.386664.

Sigler, A., Oh, W. C., Imig, C., Altas, B., Kawabe, H., Cooper, B. H., et al. (2017). Formation and Maintenance of Functional Spines in the Absence of Presynaptic Glutamate Release. Neuron 94, 304-31 I. doi:I0.1016/j.neuron.2017.03.029.

Smith, K. R., Jones, K.A., Kopeikina, K. J., Burette, A. C., Copits, B. A., Yoon, S., et al. (2017). Cadherin-I0 Maintains Excitatory/Inhibitory Ratio Through Interactions with Synaptic Proteins. J. Neurosci. 37, III27-III39. doi:10.1523/ JNEUROSCI. I I53-17.20I7.

Spruston, N. (2009). “Dendritic Signal Integration,” in Encyclopedia of Neuroscience, ed. L. R. Squire (Academic Press), 445-452. doi:I0.1016/B978008045046-9.01648-X.

Stein, I. S., Gray, J. A., and Zito, K. (2015). Nonlonotropic NMDA Receptor Signaling Drives Activity-Induced Dendritic Spine Shrinkage. J. Neurosci. 35, 12303-12308. doi:10.1523/ JNEUROSCI.4289-|4.20I5.

Stokes, C. C.A., Teeter, C. M., and Isaacson, J. S. (20I4). Single dendrite-targeting interneurons generate branch-specific inhibition. Front. Neural Circuits 8, I-9. doi: I0.3389/fncir.20I4.00I39.

Stuart, G. J., and Spruston, N. (20/5). Dendritic integration: 60 years of progress. Nat. Neurosci. 


\section{|8, |7|3-172| doi:|0.1038/nn.4157.}

Subramanian, J., Michel, K., Benoit, M., and Nedivi, E. (2019). CPGI5/Neuritin Mimics Experience in Selecting Excitatory Synapses for Stabilization by Facilitating PSD95 Recruitment. Cell Rep. 28, I584-I595. doi:I0.1016/j.celrep.2019.07.012.

Südhof, T. C. (20/2). The Presynaptic Active Zone. Neuron 75, II-25. doi:10.1016/j. neuron.2012.06.012

Takeuchi, T., Duszkiewicz, A. J., and Morris, R. G. M. (20l4). The synaptic plasticity and memory hypothesis: Encoding, storage and persistence. Philos. Trans. R. Soc. B Biol. Sci. 369, I-14. doi:I0.1098/rstb.2013.0288.

Tang,A.H.,Chen,H.,Li,T.P.,Metzbower,S.R.,MacGillavry, H. D., and Blanpied, T. A. (20I6). A trans-synaptic nanocolumn aligns neurotransmitter release to receptors. Nature 536, 210-214. doi:10.1038/ nature 19058

Tatti, R., Haley, M. S., Swanson, O. K., Tselha, T., and Maffei,A.(20I7). Neurophysiology and Regulation of the Balance Between Excitation and Inhibition in Neocortical Circuits. Biol. Psychiatry 8I, 82I83I. doi:I0.1016/j.biopsych.2016.09.017.

Terauchi, A., Johnson-Venkatesh, E. M., Toth, A. B., Javed, D., Sutton, M. A., and Umemori, H. (20I0). Distinct FGFs promote differentiation of excitatory and inhibitory synapses. Nature 465, 783-787. doi:10.1038/nature0904I.

Tran-Van-Minh, A., Cazé, R. D., Abrahamsson, T., Cathala, L., Gutkin, B. S., and DiGregorio, D. A. (20I5). Contribution of sublinear and supralinear dendritic integration to neuronal computations. Front. Cell. Neurosci. 9, I-15. doi:10.3389/ fncel.20I5.00067.

Turrigiano, G. (20/2). Homeostatic Synaptic Plasticity: Local and Global Mechanisms for Stabilizing Neuronal Function. Cold Spring Harb. Perspect. Biol. 4, I-I8. doi: I0.1 I0 I/cshperspect.a005736.

Turrigiano, G. G. (2008). The Self-Tuning Neuron: Synaptic Scaling of Excitatory Synapses. Cell I35, 422-435. doi:10.1016/j.cell.2008.10.008.

Turrigiano, G. G., Leslie, K. R., Desai, N. S., Rutherford, L. C., and Nelson, S. B. (1998). Activitydependent scaling of quantal amplitude in neocortical neurons. Nature 39I, 892-896. doi: $10.1038 / 36103$

Tyagarajan, S. K., and Fritschy, J.-M. (20I4). Gephyrin:A master regulator of neuronal function? Nat. Rev. Neurosci. I5, I4I-I56. doi:10.1038/nrn3670.

Uezu, A., Kanak, D. J., Bradshaw, T. W. A., Soderblom, E. J., Catavero, C. M., Burette, A. C., et al. (20I6). Identification of an elaborate complex mediating postsynaptic inhibition. Science 353, II $23-$ I I 29. doi:I0.I I26/science.aag082I.

Ujfalussy, B. B., Makara, J. K., Branco, T., and Lengyel, M. (20I5). Dendritic nonlinearities are tuned for efficient spike-based computations in cortical circuits. Elife 4, I-5I. doi: I0.7554/eLife. 10056.

Ulrich, D., and Bettler, B. (2007). GABA ${ }_{B}$ receptors: synaptic functions and mechanisms of diversity. Curr. Opin. Neurobiol. 17,298-303. doi: 10.1016/j. conb.2007.04.00I.

van Versendaal, D., Rajendran, R., Saiepour, M. H., Klooster, J., Smit-Rigter, L., Sommeijer, J.-P., et al. (20I2). Elimination of Inhibitory Synapses Is a Major Component of Adult Ocular Dominance Plasticity. Neuron 74, 374-383. doi:10.1016/j. neuron.2012.03.0I5

Vervaeke, K., Lörincz, A., Nusser, Z., and Silver, R. A. (20/2). Gap Junctions Compensate for Sublinear Dendritic Integration in an Inhibitory Network. Science 335, 1624-1629. doi:10.1126/ science.1215101.

Villa, K. L., Berry, K. P., Subramanian, J., Cha, J.W., Oh,W. C., Kwon, H.-B., et al. (2016). Inhibitory Synapses Are Repeatedly Assembled and Removed at Persistent Sites In Vivo. Neuron 89, 756-769. doi:10.1016/j.neuron.2016.01.010.

Vogels, T. P., and Abbott, L. F. (2009). Gating multiple signals through detailed balance of excitation and inhibition in spiking networks. Nat. Neurosci. 12, 483-49I. doi: I0.1038/nn.2276.

Vogels, T. P., Sprekeler, H., Zenke, F., Clopath, C., and Gerstner,W. (20I I). Inhibitory Plasticity Balances Excitation and Inhibition in Sensory Pathways and Memory Networks. Science 334, I569-1 573. doi:I0.1 | 26/science. I2II095.

Wamsley, B., and Fishell, G. (20I7). Genetic and activity-dependent mechanisms underlying interneuron diversity. Nat. Rev. Neurosci. 18, 299-309. doi:I0.1038/nrn.2017.30.

Wierenga, C. J. (2017). Live imaging of inhibitory axons: Synapse formation as a dynamic trialand-error process. Brain Res. Bull. 129, 43-49. doi:10.1016/j.brainresbull.2016.09.018. 
Wierenga, C. J., Becker, N., and Bonhoeffer, T. (2008). GABAergic synapses are formed without the involvement of dendritic protrusions. Nat. Neurosci. II, I044-1052. doi: 10.1038/nn.2180.

Wilson, D. E., Whitney, D. E., Scholl, B., and Fitzpatrick, D. (2016). Orientation selectivity and the functional clustering of synaptic inputs in primary visual cortex. Nat. Neurosci. 19, 1003-1009. doi: $10.1038 / n n .4323$.

Wondolowski, J., and Dickman, D. (20I3). Emerging links between homeostatic synaptic plasticity and neurological disease. Front. Cell. Neurosci. 7, I-9. doi:I0.3389/fncel.20|3.00223.

Xue, M., Atallah, B. V., and Scanziani, M. (20|4). Equalizing excitation-inhibition ratios across visual cortical neurons. Nature 51 I, 596-600. doi:10.1038/nature 13321 .

Yang, G., Pan, F., and Gan, W.-B. (2009). Stably maintained dendritic spines are associated with lifelong memories. Nature 462, 920-924. doi:I0.1038/nature08577. 


\title{
Single synapse LTP: a matter of context?
}

\author{
Dennis L. H. Kruijssen and Corette J.Wierenga
}

Department of Biology, Science for Life, Utrecht University, Utrecht, The Netherlands 


\section{Abstract}

The most commonly studied form of synaptic plasticity is long-term potentiation (LTP). Since 15 years, it is possible to induce structural and functional LTP in dendritic spines using two-photon glutamate uncaging, allowing for studying the signaling mechanisms of LTP with single synapse resolution. In this review, we compare different stimulation methods to induce single synapse LTP and discuss how LTP is expressed.We summarize the underlying signaling mechanisms that have been studied with high spatiotemporal resolution. Finally, we discuss how LTP in a single synapse can be affected by excitatory and inhibitory synapses nearby. We argue that single synapse LTP is highly dependent on context: the choice of induction method, the history of the dendritic spine and the dendritic vicinity crucially affect signaling pathways and expression of single synapse LTP. 


\section{Introduction}

Synaptic plasticity is the fundamental cellular correlate of learning. By the strengthening and weakening of specific connections, information processing in the brain is changed and memories are formed. The most studied form of plasticity is long-term potentiation (LTP). As first identified in the rabbit brain by Bliss and Lømo (Bliss and Lømo, 1973), repeatedly stimulating synapses can lead to long lasting enhancement of synaptic strength. This phenomenon has been extensively studied and characterized in a variety of brain regions and species. The majority of studies use electrical stimulation of axon bundles to induce and measure LTP in brain slices. LTP can also be induced pharmacologically by applying for example an NMDA receptor agonist. These approaches induce LTP in bulk: many synapses on dendritic branches of multiple neurons are potentiated at the same time. Electrophysiological recordings and biochemical analysis of the underlying signaling pathways have provided significant insights into the mechanisms of LTP (Bliss and Collingridge, 2013; Citri and Malenka, 2008; Diering and Huganir, 2018; Herring and Nicoll, 20I6; Malenka and Bear, 2004; Mayford et al., 2012; Nicoll, 2017; Sjöström et al., 2008). However, this way of inducing LTP does not reflect the physiological situation very well. Under physiological conditions, synaptic inputs are usually not synchronously active in such large numbers and synaptic plasticity presumably takes place at a scale of individual or small groups of synapses.

The development of two-photon glutamate uncaging almost 20 years ago (Ellis-Davies, 2019; Matsuzaki et al., 200I, 2004) made it possible to activate and potentiate individual synapses. Using a caged compound of the main excitatory neurotransmitter, individual excitatory synapses on spines can be activated with focused laser light at a near-physiological spatial and temporal scale (Matsuzaki et al., 200I) and plasticity can be induced by repetitive stimulation (Matsuzaki et al., 2004). Since then, many studies have used two-photon glutamate uncaging to study the induction, expression and signaling pathways of LTP in single synapses. These studies significantly have improved our understanding of the mechanisms underlying LTP at the single synapse level. However, differences and disagreements between studies also reveal the limitations of our current understanding of single synapse LTP.

The goal of this review is to summarize and compare studies that used two-photon glutamate uncaging to gain insight into single synapse LTP signaling pathways. We will compare different methods to induce LTP in single synapses and discuss how the choice of LTP induction protocol may affect LTP expression and signaling pathways. We will summarize the signaling pathways that are triggered in a single spine during LTP induction using two-photon uncaging and discuss the possibility that multiple LTP pathways may exist, which can be differentially activated depending on the experimental conditions. Finally, we discuss how LTP at a single synapse can affect plasticity at other excitatory and inhibitory synapses on the same dendrite, suggesting that potentiation of an individual synapse should always be considered in the context of its direct dendritic vicinity. 


\section{Induction of single synapse LTP}

Two-photon microscopy (Denk et al., 1990; Masters and So, 2004) utilizes the physical principle of two-photon excitation: fluorescent proteins are excited only in a femtolitersized volume inside the laser beam focus, where the laser light intensity is high enough for excitation by two coincident photons (Svoboda and Yasuda, 2006; Zipfel et al., 2003). Individual long wavelength photons have low energy, which means that out-of-focus laser light causes minimal photodamage. In addition, long wavelength light can penetrate deep into tissue without scattering, allowing to perform live two-photon imaging of small structures such as dendritic spines up to I mm deep into living brain tissue (Denk and Svoboda, 1997; Helmchen and Denk, 2005). With the same precision, the two-photon principle allows for precise photolysis of "caged compounds" - biologically active molecules that are inert until exposed to the right wavelength of light (Soeller and Cannell, 1999). The development of MNI-glutamate, a caged compound of the main excitatory neurotransmitter which has a high two-photon cross section, allowed stimulation of single excitatory synapses (Matsuzaki et al., $200 \mathrm{I}$ ) and induction of plasticity at individual spines (Matsuzaki et al., 2004). The development of several Förster Resonance Energy Transfer (FRET) probes that can detect the activity of signaling molecules on the level of the single spine allowed studying the underlying pathways of LTP with greater detail than ever before (Nakahata and Yasuda, 20I8; Ueda et al., 20I3; Yasuda, 2012). With these technological advancements, it is now possible to elucidate the mechanisms that are involved in LTP on the level of single excitatory synapses.

The first study to report single synapse LTP was performed by Matsuzaki and colleagues (Matsuzaki et al., 2004). Upon performing repeated glutamate uncaging on single dendritic spines, the stimulated spines rapidly grew and remained enlarged for up to 100 minutes, while unstimulated spines on the same dendrites were unaffected. The authors furthermore showed that spine growth crucially depended on NMDA receptor activation and was similar to spine growth after electrical stimulation. Spine growth was accompanied by a corresponding increase in AMPA receptor-mediated postsynaptic currents, linking growth of the spine head with functional plasticity of the excitatory synapse. Since the pioneering work by Matsuzaki and colleagues, the two-photon glutamate uncaging technique was quickly adopted by the LTP field and multiple labs have performed single synapse LTP experiments since then. A major benefit of using glutamate uncaging to study LTP is the high spatial and temporal precision of the stimulus. As presynaptic stimulation is no longer required, it allows for isolating the postsynaptic component of LTP.

Single synapse LTP is generally induced by repeated uncaging pulses. The repeated activation of postsynaptic glutamate receptors results in calcium influx, most prominently via NMDA receptors, which triggers plasticity at the stimulated spine. Induction protocols for LTP differ in several aspects, which may significantly influence downstream signaling and LTP expression. The number of uncaging pulses typically ranges from 30 to 60 , and the stimulation frequency usually lies between 0.5 and $2 \mathrm{~Hz}$. Both these parameters will likely affect the total amount of calcium entering the postsynaptic cell and the level of activation of downstream calcium sensing proteins (Fujii et al., 2013). The duration of a single uncaging pulse typically lies between 0.5 and $6 \mathrm{~ms}$. The pulse duration determines the time receptors are exposed to glutamate, as well as the total amount of glutamate that is uncaged, affecting the duration and level of activation of glutamate receptors (AMPA receptors and NMDA receptors) in the postsynapse. The uncaging beam is typically aimed $0.5 \mu \mathrm{m}$ from the spine head to prevent photodamage to the spine. The distance between the location of glutamate release and the 
spine will impact the diffusion time of glutamate to the receptors. While glutamate uncaging is highly local, especially during strong stimulation glutamate spillover to extrasynaptic receptors and presynaptic receptors (such as metabotropic glutamate receptors) is likely to occur (Chalifoux and Carter, 20II; Rusakov and Kullmann, 1998).
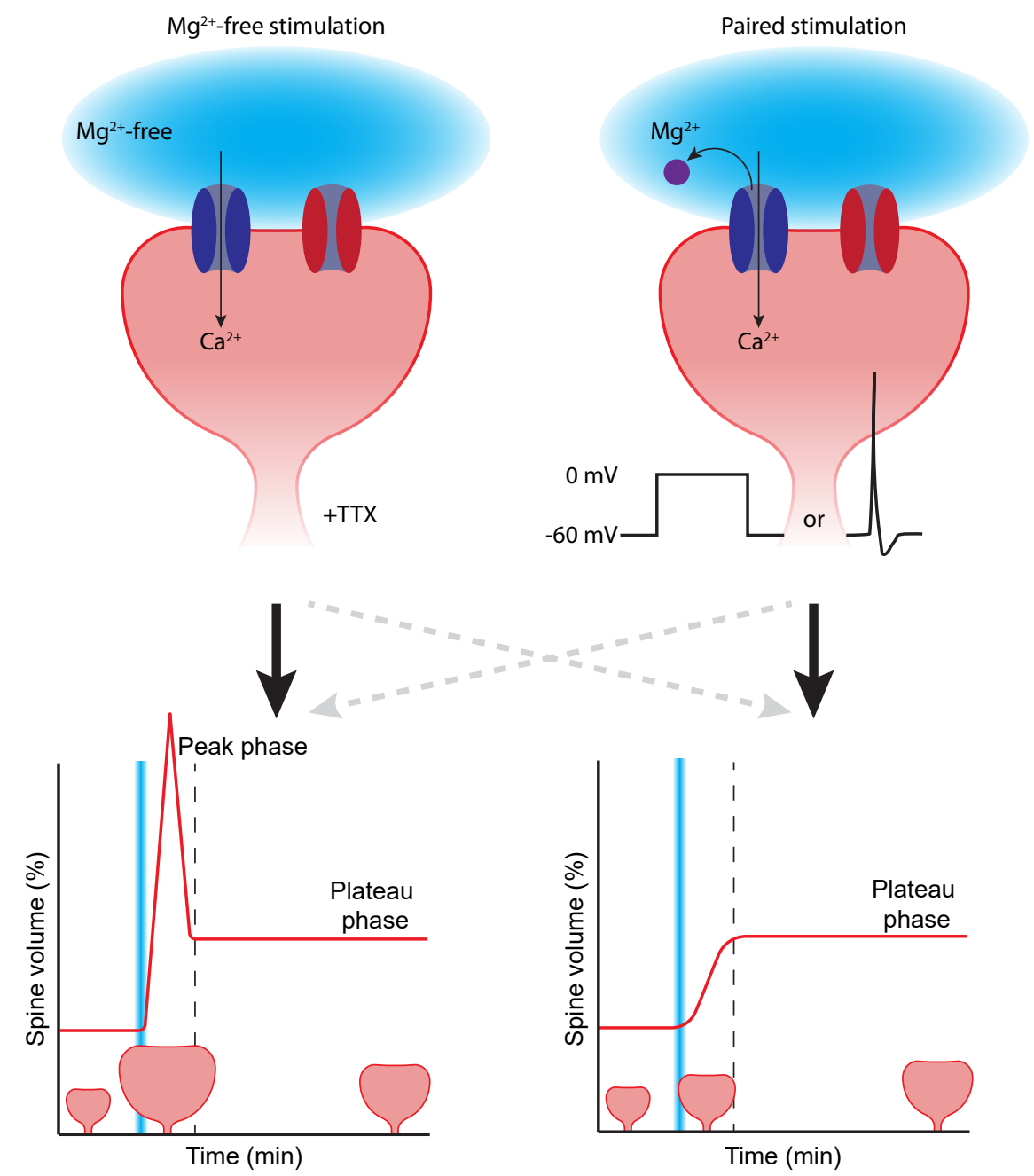

Figure 1. The choice of LTP induction method can affect spine growth

To induce spine growth and functional LTP in single synapses, activation of NMDA receptors (dark blue) is required. Removing the magnesium block (purple) from the NMDA channel pore can be achieved in two ways:

Left: glutamate uncaging is performed in the absence of extracellular magnesium $\left(\mathrm{Mg}^{2+}-\right.$ free). In this case, tetrodotoxin (TTX) is added to prevent aberrant plasticity due to spontaneous activity. This type of stimulation typically induces rapid, strong initial growth (peak phase), after which the spine volume stabilizes at a lower level (plateau phase).

Right: In paired protocols, two-photon glutamate uncaging (blue) is paired with depolarization (in voltage clamp by increasing the holding potential, or in current clamp by inducing a backpropagating action potential). Paired stimulation typically leads to a gradual growth of the dendritic spine over time.

AMPA receptors in the spine head are depicted in red. The dashed gray lines reflect that the correlation between stimulation protocol and temporal profile of spine growth is not absolute. 
NMDA receptor activation is one of the crucial events for LTP to occur, and different methods are used to ensure NMDA receptor activation during glutamate uncaging at spines (Figure I). Here, we roughly divide these protocols in two categories. The first category is based on the protocol by Matsuzaki and colleagues. To achieve NMDA receptor activation, glutamate uncaging is performed in absence of extracellular magnesium ions to remove blockage of the channel pore (Harward et al., 20 I 6; Lee et al., 2009; Oh et al., 20 I 5; Patterson et al., 20l0; Tanaka et al., 2008; Tønnesen et al., 20l4). Caged compounds are known to

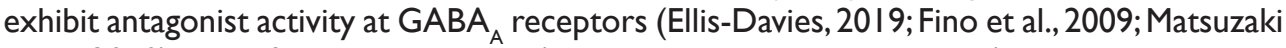
et al., 20I0). Therefore, tetrodotoxin (TTX, a sodium channel blocker) is usually added to the bath solution under magnesium-free conditions to prevent epileptiform-like activity and unwanted plasticity. The second category of protocols pairs glutamate uncaging with postsynaptic depolarization or postsynaptic action potentials to relieve the magnesium block from the NMDA receptors. This type of protocol typically requires electrical access to the postsynaptic cell via a patch clamp electrode. In voltage clamp experiments, the cell is depolarized (typically to $0 \mathrm{mV}$ ) while glutamate is uncaged at a spine (Harvey and Svoboda, 2007; Lee et al., 2009; Matsuzaki et al., 2004). In current clamp experiments, current is injected to induce action potential firing while glutamate is uncaged at a spine (Hayama et al., 20 I3; Tanaka et al., 2008). Alternatively, all-optical uncaging LTP experiments can be performed by pairing optogenetically induced postsynaptic depolarization with glutamate uncaging (Zhang et al., 2008).

The majority of studies have used magnesium-free protocols, which has the great advantage that electrical access to the postsynaptic cell is not required and the studied neuron can be left unperturbed. However, performing experiments in magnesium-free extracellular solution is far from physiological: NMDA receptors are constantly "primed" for activation and addition of TTX is required to block all spontaneous electrical activity. Furthermore, the absence of magnesium could affect several other cellular processes that require magnesium (de Baaij et al., 20I5). Paired protocols mimic physiological conditions more accurately. Under physiological conditions, the magnesium block will be relieved by depolarization of the postsynaptic membrane (Gambino et al., 20l4). An additional advantage is that the use of patch clamp electrophysiology allows recording of the uncaging-induced excitatory postsynaptic current (uEPSC). This way, the laser power can be tuned to induce uEPSCs with amplitudes that are similar to single synapse EPSCs (typically $\sim 10-20 \mathrm{pA}$ ) to mimic synaptic glutamate levels (Harvey and Svoboda, 2007; Hill and Zito, 20I3; Lee et al., 2009; Matsuzaki et al., 200I, 2004; Steiner et al., 2008). However, the use of electrophysiology makes paired protocols more invasive. Signaling molecules that are required for LTP may "wash out" while perfusing the cell with internal solution from the patch pipette, thereby reducing or abolishing the ability to induce LTP (Malinow and Tsien, 1990; Matsuzaki et al., 2004; Tanaka et al., 2008).

In conclusion, the choice of protocol involves several practical and biological considerations, such as the need for patch clamp electrophysiology, washout of signaling molecules, and resemblance of the physiological situation. It is important to realize that these protocols are not completely interchangeable: in the next section, we will discuss how the induction protocol may affect the magnitude and temporal profile of LTP expression. 


\section{Expression of single synapse LTP}

Inducing LTP in a synapse has two major effects: the number of postsynaptic AMPA receptors is increased and the spine volume is enlarged. After LTP, a presynaptic stimulus will induce a postsynaptic current with larger amplitude than before. This is largely due to an increase of AMPA receptors in the postsynaptic membrane (Huganir and Nicoll, 20I3; Kessels and Malinow, 2009; Moretto and Passafaro, 2018). Matsuzaki and colleagues showed that also in single potentiated spines, the AMPA receptor-mediated currents increase within minutes after stimulation (Matsuzaki et al., 2004). Many LTP induction paradigms, such as highfrequency stimulation, theta burst stimulation and optical stimulation of afferents lead to persistent spine growth, which was shown by fluorescence imaging (De Roo et al., 2008; Lang et al., 2004; Okamoto et al., 2004;Wiegert et al., 2018) and electron microscopy (Bourne and Harris, 20I I; Buchs and Muller, 1996;Van Harreveld and Fifkova, 1975). In vivo, spine volumes fluctuate and spines are continuously formed and removed (Berry and Nedivi, 2017; Caroni et al., 20/2). Spine dynamics are enhanced after experience and are thought to support long-lasting changes in neural circuits during experience-dependent plasticity (Hofer et al., 2009; Holtmaat et al., 2006; Roberts et al., 2010). For instance, specific spines grow during a motor learning task, and inducing shrinkage of these spines disrupts the acquired motor skill (Hayashi-Takagi et al., 20I5). Spine growth is largely attributed to remodeling of actin, which is highly enriched in spines. When a spine is potentiated, polymerization of actin in the spine head leads to more filamentous actin and a bigger spine (Bosch and Hayashi, 2012; Harvey et al., 2008; Matsuzaki et al., 2004; Nakahata and Yasuda, 20I8; Okamoto et al., 2004). These morphological changes (actin polymerization and spine growth) and functional changes (increase in AMPA receptors) are often correlated, but might be regulated independently.

To monitor the expression of LTP in individual synapses, the increase in amplitude of the uncaging-induced excitatory postsynaptic current (uEPSC) can be quantified. The uEPSC at a spine can go up 40-120\% within minutes after LTP induction (Harvey and Svoboda, 2007; Matsuzaki et al., 2004; Steiner et al., 2008; Tønnesen et al., 20I4). While quantifying uEPSC increase is a useful method to assess functional LTP, it can be technically challenging. Other than on the strength of the synapse, the uEPSC amplitude also depends on the laser power at the uncaging location, the local caged glutamate concentration, and the distance of the uncaging spot to the postsynaptic density, all of which are challenging to keep stable at growing spines during the experiment. Furthermore, electrical access to the postsynaptic cell is required. Quantification of the morphological changes of the spine head is therefore often used as an alternative measure.

Spine growth can be quantified using two-photon microscopy images of the stimulated spine over time. Depending on the initial size and the protocol used, spine heads show (transient) growth up to 200-400\% (Bosch et al., 2014; Matsuzaki et al., 2004; Murakoshi et al., 2017; Tønnesen et al., 2014). Spine size correlates strongly with synapse strength under resting conditions in vitro (Matsuzaki et al., 200I; Noguchi et al., 2005; Zito et al., 2009) and in vivo (Noguchi et al., 20II). Because of this strong correlation, as well as the technical challenges of quantifying uEPSC amplitude over time, spine growth is often taken as a proxy for functional LTP.The correlation between size and function is however not absolute: morphological and functional changes might not match perfectly in the first hour after LTP induction (Bosch et al., 20I4; Meyer et al., 20I4) and functional LTP can also occur in the absence of spine head growth (Araya et al., 2014). It is important to mention that the high laser power used for glutamate uncaging can induce photodamage and swelling of the spine head when the laser 
beam is aimed too close to the spine head. Swelling due to photodamage could potentially confound actual spine growth due to LTP, but can be prevented by aiming the laser beam $\sim 05 .-\mathrm{I} \mu \mathrm{m}$ away from the spine head.

\section{Other morphological changes}

The increase in spine head size is not the only morphological change upon LTP. Several studies have reported shorter and/or thicker spine necks after LTP induction (Araya et al., 20I4; Bosch et al., 20I4; Tanaka et al., 2008; Tønnesen et al., 20I4). These changes in spine neck geometry seem to be consistent with an increase in electrical coupling (Araya et al., 2006, 20I4; Tønnesen et al., 2014) and may provide a mechanism for synaptic strengthening independent of AMPA receptor regulation. In addition, glutamate uncaging may induce remodeling of the extracellular space, possibly via glial responses (Tønnesen et al., 2018).

Glutamate uncaging bypasses the need of activating glutamate release of the presynaptic terminal and allows isolation of the postsynaptic component of LTP. However, the presynaptic bouton is probably also affected by glutamate uncaging. After a putative LTP-inducing uncaging protocol, boutons increase their size by $\sim 50 \%$ gradually over the course of I-3 hours, maintaining the correlation between bouton size and spine size (Meyer et al., 20I4).

It has also been reported that repeated glutamate uncaging on the dendrite can induce the formation of a new dendritic spine at the uncaging location within seconds, which can become functional within 30 minutes (Hamilton et al., 20 I2; Kwon and Sabatini, 20 I I). In a different study it was shown that new spines rapidly mature and become functional (Zito et al., 2009). New spines have the capacity to grow upon glutamate uncaging, which significantly increases their persistence (Hill and Zito, 20I3).

\section{Variability in spine growth}

There is a remarkable level of variability in the reported time course and magnitude of spine growth between studies, even within the same brain region and cell type (Bosch et al., 2014; Tanaka et al., 2008). Many studies report an initial peak (or transient phase) of a few minutes in which the spine grows drastically. This peak growth can range from $100-400 \%$. This phase is then followed by a plateau (or sustained phase) where the spine growth declines and stabilizes, typically at 50-100\% (Bosch et al., 20I4; Harvey and Svoboda, 2007; Harward et al., 2016; Oh et al., 20I5; Patterson et al., 2010; Tanaka et al., 2008; Tønnesen et al., 20I4). Other studies report a gradual spine growth over the course of $5-10$ minutes, which then stabilizes at a plateau, without a significant peak (Harvey and Svoboda, 2007; Hayama et al., 20 I 3; Hu et al., 2019; Tanaka et al., 2008). Even when comparing studies that show a similar temporal pattern of spine growth, peak and plateau magnitudes often vary significantly. One could wonder to what extent extreme peak spine growth resembles the physiological situation.

Technical differences, such as differences in quantification methods and model systems could partially explain this remarkable variability, but other factors may be more vital. First of all, the initial size of the spine before induction of LTP matters: small spines have a larger growing capacity than spines that are already larger to begin with (Matsuzaki et al., 2004; Tanaka et al., 2008). It has even been suggested that the large spines cannot grow upon stimulation at all (Matsuzaki et al., 2004; Tanaka et al., 2008). Although it is difficult to compare initial spine size between studies, a difference in initial spine size may explain some of the observed differences in spine growth magnitude. 
More importantly, the choice of LTP induction protocol will crucially affect the magnitude and time course of spine growth. This was first observed by Tanaka and colleagues. When they paired glutamate uncaging with backpropagating action potentials, it lead to a gradual growth of the spine, reaching close to $150 \%$ growth. However, when they performed glutamate uncaging in absence of extracellular magnesium, spine growth showed an initial peak in which spine volume reached 2 -fold growth (100\%), after which spine growth declined to reach a plateau phase at $50 \%$ (Tanaka et al., 2008). Similarly, Harvey \& Svoboda report a gradual spine growth of $80 \%$ when using a paired protocol. A similar magnesium-free protocol results in $175 \%$ peak growth, declining to a plateau at $75 \%$ growth (Harvey and Svoboda, 2007). These studies clearly suggest that different induction protocol activate different intracellular signaling pathways, resulting in differences in spine growth. Typically, magnesium-free induction protocols lead to peak-plateau growth, while paired protocols often induce gradual spine growth (Figure I; although this correlation is not absolute (Lee et al., 2009; Matsuzaki et al., 2004; Zhang et al., 2008)).

Why do different induction protocols lead to such remarkable differences in spine growth? The choice of induction protocol likely affects how downstream signaling pathways are activated. This already occurs at the level of calcium concentration elevation, the key signal for LTP. While calcium influx is typically restricted to the spine head in magnesium-free stimulation protocols, paired protocols also cause an increase of calcium concentration in the dendritic shaft (see below). This differential spatial calcium profile may also lead to differential activation of downstream signaling molecules and it is interesting to speculate how these could be linked to the peak and plateau phases of spine growth. For instance, the study by Tanaka and colleagues showed that the paired protocol involved BDNF signaling and protein synthesis to induce spine growth, while spine growth was independent of BDNF in the magnesium-free protocol (Tanaka et al., 2008). However, a more recent study observed that BDNF also affects spine growth after a magnesium-free protocol (Harward et al., 2016). These data suggest that there is not a single universal mechanism for the expression LTP in spines. Multiple modes of LTP may exist, and different protocols may activate different signaling mechanisms. We will discuss these signaling pathways in the next sections. We will first describe which pathways are activated when LTP is induced in single spines, followed by a discussion on how signaling pathways between nearby synapses can interact. 


\section{Single synapse LTP signaling pathways}

In this section, we discuss the signaling pathways that are activated when a single spine is potentiated. Expression of LTP has been extensively examined using chemical or electrical LTP induction, in which multiple synapses are stimulated in many neurons are activated simultaneously and signaling pathways are triggered in a large part of the neuron. These studies have established that calcium influx through NMDA receptors and subsequent activation of CaMKII are essential for LTP. Downstream signaling pathways eventually lead to actin remodeling and the insertion of AMPA receptors, resulting in a stronger synapse. Here we limit our discussion to studies using two-photon glutamate uncaging to induce LTP in a single synapse. By inducing potentiation in a single synapse, it is possible to study the activation of molecules in LTP signaling pathways with the highest temporal and spatial detail.

\section{Glutamate receptors}

Glutamate uncaging on a dendritic spine activates AMPA receptors and NMDA receptors in the postsynaptic density (although glutamate receptors can also be found extrasynaptically and presynaptically (Bouvier et al., 2018; Parsons and Raymond, 20I4)). AMPA receptors mainly conduct sodium and potassium ions and are largely responsible for synaptic membrane depolarization in the spine. Binding of glutamate to NMDA receptors is usually not sufficient to open the channel, as they are blocked by magnesium. Only when the postsynaptic membrane is sufficiently depolarized, during AMPA receptor activation, a backpropagating action potential or a dendritic spike, the magnesium block is relieved and NMDA channels open. When NMDA receptors are activated, it leads to the rapid influx of calcium ions through the channel pore into the dendritic spine. Many studies have demonstrated that NMDA receptor activation is required for the growth of single spines (Harvey and Svoboda, 2007; Matsuzaki et al., 2004; Tang and Yasuda, 20I 7; Zhai et al., 20I3).

Not all spines contain both AMPA receptors and NMDA receptors. AMPA receptor content is correlated to spine size, and the smallest spines can be silent, meaning that they contain no AMPA receptors and therefore no current can be measured when the spine is exposed to glutamate. These silent spines however do contain NMDA receptors (Béïque et al., 2006; Busetto et al., 2008). This allows these spines to undergo LTP by growing and recruiting AMPA receptors.

Besides ionotropic glutamate receptors, dendritic spines also contain group I metabotropic glutamate receptors (mGluRs). These mGluRs are enriched immediately next to the postsynaptic density (Scheefhals and MacGillavry, 2018). When glutamate uncaging is performed at a dendritic spine, it is likely that mGluRs will also be activated, especially when long uncaging pulses or many repetitions are used. When the metabotropic glutamate receptors are blocked during the induction of single synapse LTP, spine growth typically remains intact (Bosch et al., 20 I4; Colgan et al., 20I8; Matsuzaki et al., 2004; Zhai et al., 20I3), suggesting they do not play a major role in LTP induction.

\section{Calcium}

Calcium entering the spine via NMDA receptor activation is considered the key signal to trigger LTP. During a single synapse LTP induction protocol, each uncaging stimulus leads to a brief influx of calcium into the dendritic spine (Colgan et al., 20 I8; Lee et al., 2009; Zhai et 
al., 20I3). There is a tight inverse correlation between spine head volume and calcium levels: uncaging on a smaller spine leads to a higher calcium concentration (Noguchi et al., 2005; Sobczyk et al., 2005). This can partly be explained by geometric differences, but different subunit composition of NMDA receptors in smaller spines may also play a role (Sobczyk et al., 2005). Depending on the geometry of the spine neck (length and width), some calcium will diffuse from the spine head into the dendritic shaft (Noguchi et al., 2005; Zhai et al., 20I3).

While calcium influx through NMDA receptors is crucial for LTP induction, other sources of calcium can be involved as well. When glutamate uncaging is paired with postsynaptic depolarization, voltage-gated calcium channels in the dendrite and spine get activated (Lee et al., 2009; Müllner et al., 20I5) and this will lead to additional calcium influx. An experiment by Zhai and colleagues suggests that voltage-gated calcium channels (VGCCs) do not play a role in the induction of LTP under magnesium-free conditions, but may affect the plateau level of spine growth (Zhai et al., 20I3).

\section{Calcium-sensing proteins}

The increase of calcium concentration upon NMDA receptor activation is sensed by Calcium/ calmodulin kinase II (CaMKII) and activation of CaMKII is essential for the induction of LTP. CaMKII can associate with several structures in the spine head, such as filamentous actin and several proteins in the postsynaptic density (Hell, 20 I4; Kim et al., 20 I5; Okamoto et al., 2004). Changes in local CaMKII levels may occur after single synapse LTP induction. CaMKII concentration in the spine has been reported to temporarily drop for 5 minutes (Bosch et al., 20I4), or slightly but persistently increase after LTP induction (Zhang et al., 2008). As changes in the concentration of CaMKII are also dependent on changes in spine volume, it is important to mention that these studies use different induction protocols (magnesiumfree versus paired) and observe a different temporal pattern and amplitude of spine growth. Both studies agree that the total amount of bound (as opposed to freely diffusing) CaMKII in the spine head increases after LTP induction (Bosch et al., 20 I4; Zhang et al., 2008). It was previously shown that the amount of bound CaMKII in the spine correlates strongly with spine size and uEPSC amplitude under baseline conditions (Asrican et al., 2007), suggesting that the trapping of CaMKII in the spine head is directly related to strengthening of the spine during LTP. On longer timescales, the fraction of bound CaMKII returns to baseline (Asrican et al., 2007; Zhang et al., 2008), indicating that unbound CaMKII slowly diffuses to the spine to restore the ratio of bound/unbound CaMKII.

CaMKII is activated by calcium and the calcium-binding protein calmodulin. Calmodulin associates with and dissociates from CaMKII within seconds. The association of calmodulin and CaMKII does not accumulate during a single synapse LTP induction protocol (Chang et al., 2019). CaMKII activation however does increase with every uncaging pulse, thereby integrating multiple calcium signals. CaMKII even stays active for up to I minute after the end of the induction protocol (Chang et al., 2017, 2019; Lee et al., 2009). This accumulation and persistence of the signal can be explained by autophosphorylation (at the threonine 286 residue), allowing CaMKII to remain active after calcium/calmodulin unbinds. Autophosphorylation of CaMKII is important for LTP induction: the slower inactivation rate permits signal integration at relatively low frequency stimulation. Only at extremely high frequencies $(>8 \mathrm{~Hz}$ ), repeated stimulation can sustain CaMKII activation without autophosphorylation (Chang et al., 2017). 
CaMKII plays an important role in spine growth. Multiple studies show that pharmacological inhibition or genetic knockout of CaMKII strongly reduces the plateau phase of spine growth, while peak growth is maintained (Hedrick et al., 2016; Incontro et al., 2018; Lee et al., 2009; Matsuzaki et al., 2004; Murakoshi et al., 20II; Saneyoshi et al., 2019). Using a photoactivatable CaMKII inhibitor, Murakoshi and colleagues demonstrated that CaMKII activation is required for only one minute during LTP induction. Interestingly, both the peak and plateau of spine growth was strongly reduced when the inhibitor was activated during the entire LTP induction protocol. When the inhibitor was activated 30 seconds after the start of the induction protocol, only plateau growth was reduced while peak growth remained (Murakoshi et al., 2017). These data suggest that the peak and plateau growth require different durations of $\mathrm{CaMKII}$ activation, but are in disagreement with experiments using pharmacological inhibition of CaMKII (discussed above).

The spatial extent of CaMKII activation depends on the LTP induction protocol. In a typical magnesium-free induction protocol, CaMKII activation is mostly restricted to the spine head (Lee et al., 2009), although a small amount of active CaMKII might be found in the dendritic shaft (Chang et al., 2017). However, when glutamate uncaging is paired with postsynaptic depolarization, dendritic voltage-gated calcium channels are activated and as a result CaMKII is also strongly activated in the dendritic shaft (Lee et al., 2009).

In addition to CaMKII, the phosphatase calcineurin $(\mathrm{CaN})$ is also activated in the spine head and dendritic shaft when calcium levels increase. While CaMKII is sensitive to both the frequency and number of uncaging stimuli, $\mathrm{CaN}$ is less sensitive to stimulation frequency and mainly responds to the number of stimuli (Fujii et al., 20I3). Calcineurin activity is typically associated with spine shrinkage and synaptic depression (Hayama et al., 20 I3; Nabavi et al., 20I3; Oh et al., 20 I5; Zhou et al., 2004).

\section{GTPases: Ras, RhoA, Cdc42, Racl}

During and after LTP induction, several small GTPases are activated in the dendritic spine, via both CaMKII-dependent and -independent pathways. Small GTPases are enzymes that often function as "molecular switches" in biological signaling pathways, and play an important role in regulating the synaptic actin cytoskeleton and plasticity (Hotulainen and Hoogenraad, 20I0; Patterson and Yasuda, 20I I). Harvey and colleagues used a FRET-sensor to show that the small GTPase Ras is activated in the dendritic spine within I minute after glutamate uncaging. Activity decays substantially in 5 minutes but some Ras stays activated for at least I 5 minutes. Ras activation is partly dependent on CaMKII (Harvey et al., 2008), likely through phosphorylation of the Ras GTPase activating protein SynGAP (Araki et al., 2015), but Ras activation also depends on PI3K and PKC activity (Harvey et al., 2008). Ras presumably acts via the extracellular signal-regulated kinase ERK via the Ras-MEK pathway. ERK activation in the spine peaks within 5 minutes after LTP induction and lasts for 20 minutes (Tang and Yasuda, 2017). Ras-ERK signaling plays an important role in spine growth: interfering with Ras activation or with its downstream Raf-MEK-ERK pathway reduces the magnitude of the plateau, but not of the peak spine growth (Harvey et al., 2008; Zhai et al., 2013). When both CaMKII and the Ras-Raf-MEK-ERK pathway are inhibited, plateau spine growth is almost completely abolished, suggesting that these pathways together are responsible for the majority of spine growth in the plateau phase (Harvey et al., 2008).

RhoA, a member of the Rho subfamily of GTPases is also activated in the stimulated spine within 30 seconds upon LTP induction. While the level of activation largely decays within 
5 minutes, some activation remains for 30 minutes. Another Rho GTPase family member, Cdc42, shows similar activation kinetics. RhoA and Cdc42 activation is partially dependent on CaMKII signaling. Functional LTP is completely abolished when RhoA or Cdc42 are inhibited. Inhibition of RhoA or its downstream effector Rock reduces both the peak phase and the plateau phase of spine growth, while interfering with Cdc42 or its downstream effector Pak affects plateau phase spine growth only (Murakoshi et al., 20I I). Experiments by Hedrick and colleagues suggest that $\mathrm{Cdc} 42$ activation can be downstream from autocrine BDNF signaling (see below), while RhoA is activated independently (Hedrick et al., 2016).

A third Rho GTPase family member Racl is also activated rapidly in the dendritic spine upon LTP induction, and also partly in a CaMKII and BDNF-dependent manner. Racl shows stronger sustained activation than RhoA and Cdc42. Interfering with Racl signaling significantly reduces both peak spine growth and the plateau phase of spine growth (Hedrick et al., 2016). Recently, it was shown that sustained activation of Racl is regulated by the guanine nucleotide exchange factor Tiam I. Tiam I forms a complex with activated CaMKII and both proteins reciprocally keep each other active. Interfering with Tiam I or the complex formation between CaMKII and Tiam I significantly affects spine growth (Saneyoshi et al., 2019).

Together, the picture emerges that glutamate uncaging induces spine growth and functional LTP via multiple, and partially overlapping, GTPase pathways (Nakahata and Yasuda, 2018).

\section{PKC and PKA signaling}

Classical protein kinase C (PKC) family proteins are typically activated in the presence of calcium and the lipid diacylglycerol (DAG) (Lipp and Reither, 20I I). It has been shown that specifically PKC $\alpha$ mediates the plateau phase of spine growth (Colgan et al., 2018). PKC activation occurs in the dendritic spine and is extremely rapid: PKC is activated after every uncaging pulse, but has already decayed by the time of the next uncaging pulse (at $0.5 \mathrm{~Hz}$ ). Blocking calcium influx through NMDA receptors completely abolishes PKC activation, and PKC activation and spine growth are reduced when the production of DAG by Phospholipase C (PLC) is inhibited. During LTP induction, PLC is activated by autocrine BDNF-TrkB signaling (see below), and not by mGluR activation (Colgan et al., 20 I8).

Another important kinase, protein kinase A (PKA), seems to play a modulatory role in LTP (Blitzer et al., 1998; Esteban et al., 2003; Man et al., 2007). PKA activity depends on cyclic AMP levels and is downstream of a variety of G-protein coupled receptors. Single synapse LTP induction leads to rapid activation of PKA in the spine, which decays back to baseline in 5 minutes. Interestingly, PKA activation was found to be downstream of NMDA receptor activation (Tang and Yasuda, 20 I7). LTP does not require PKA activation, but PKA activation can boost single synapse LTP (Govindarajan et al., 20I I; Yagishita et al., 20I4). However, PKA activation originating from a single stimulated spine may not be sufficient for this boosting effect and more global PKA activation, for instance via dopaminergic neuromodulatory signals (Yagishita et al., 20I4), may be required.

\section{Actin}

Actin is the major structural component of the dendritic spine and spine growth requires actin remodeling. Matsuzaki and colleagues already showed that single spine growth is prevented when actin polymerization is prevented by the actin monomer sequestering drug 
Latrunculin A (Matsuzaki et al., 2004). In resting conditions, two pools of actin can be found in the dendritic spine: a highly dynamic pool located at the tip of the spine head, and a very stable pool at the base of the spine. After LTP induction, a third "enlargement" pool appears, and this pool seems to be responsible for spine growth (Honkura et al., 2008).

Upon LTP induction, the amount of actin in the spine and several actin-interacting proteins (Arp2/3, profilin, Aipl, drebrin, $\alpha$-actinin, cofilin) increases in parallel with spine growth (Bosch et al., 2014). Some of these proteins (Arp2/3, Aipl, actin, cofilin) increase rapidly during peak growth and the concentration of cofilin in the spine head remains elevated for at least 30 minutes. Upon LTP induction, cofilin is phosphorylated by LIM kinase, which is downstream from the Cdc42-Pak and RhoA-Rock pathways discussed above (Bosch et al., 2014). Phosphorylation of cofilin is required for the peak and plateau phases of spine growth (Noguchi et al., 2016). In the first few minutes, phosphorylated cofilin presumably severs actin filaments and thereby boosts the nucleation of new actin filaments and branching by $\operatorname{arp} 2 / 3$ resulting in spine growth. After this initial phase, cofilin is dephosphorylated again and can decorate actin filaments, thereby stabilizing them. In absence of cofilin, the plateau phase of spine growth is abolished (Bosch et al., 2014).

Interestingly, during baseline conditions CaMKII associates with actin filaments in the spine head.When calcium flows into the spine head and activates CaMKII, autophosphorylation of CaMKII causes it to dissociate from filamentous actin, allowing binding of cofilin and other actin regulators to remodel the actin cytoskeleton. After dephosphorylation, CaMKII quickly binds and thereby stabilizes actin filaments. It has been suggested that the rapid and transient ( I minute time window) dissociation of CaMKII from filamentous actin allows the rapid and transient peak spine growth observed in some studies (Kim et al., 20I5). Preventing CaMKII F-actin dissociation strongly reduces functional LTP in slices and strongly reduces fear learning in vivo (Kim et al., 2015, 20I9).

\section{Postsynaptic density and AMPA receptors}

Within minutes after single synapse LTP induction, synaptic strengthening is expressed as an increase in the amount of AMPA receptors on the spine surface (Bosch et al., 20 I4; Chiu et al., 20I7; Makino and Malinow, 2009; Patterson et al., 20I0; Soares et al., 20I7) and can be measured by an increase in AMPA receptor-mediated currents (Harvey and Svoboda, 2007; Matsuzaki et al., 2004; Steiner et al., 2008; Tønnesen et al., 2014). The increase of AMPA receptors in the postsynaptic density involves receptor phosphorylation (Boehm et al., 2006) and mainly occurs via lateral diffusion in the membrane, but exocytosis of AMPA receptor-containing vesicles also contributes (Chiu et al., 2017; Choquet, 20I8; Makino and Malinow, 2009; Patterson et al., 2010). A local increase of exocytosis rate occurs during LTP induction, which seems partially dependent on Ras-ERK-mediated, but CaMKII-independent, pathways. CaMKII signaling is likely involved in anchoring of AMPA receptors to spines (Patterson et al., 20l0).

The postsynaptic density (PSD) consists of a cluster of proteins close to the postsynaptic membrane. Important PSD proteins such as PSD95, Homer and Shank act as a scaffold to position and anchor ionotropic and metabotropic glutamate receptors (Scheefhals and MacGillavry, 2018). Remodeling of the PSD during LTP is a complex, multi-step process. Under basal conditions, the size of the PSD strongly correlates with the size of the spine head. After LTP induction, the postsynaptic density increases in size, but components arrive in the spine with a delay compared with the rapid AMPA receptor insertion (Bosch et al., 
20I4; Meyer et al., 20I4; Steiner et al., 2008). In some spines, transient spine growth can be observed after glutamate uncaging, which returns to baseline after $\sim 2$ hours without any changes to the PSD (Meyer et al., 20I4). After successful single synapse LTP, it takes at least one hour for the correlation between PSD and spine size to restore (Bosch et al., 2014; Meyer et al., 2014) .

\section{Protein synthesis}

Spine growth can occur in the absence of protein synthesis (Harvey and Svoboda, 2007; Harward et al., 2016), but some single synapse LTP induction protocols require synthesis of new proteins. Tanaka and colleagues showed that when single synapse LTP is induced in low extracellular magnesium, spine growth is independent of protein synthesis. However, when a similar induction protocol is paired with postsynaptic spiking in physiological levels of magnesium spine growth is strongly dependent of protein synthesis (Tanaka et al., 2008). A more recent study showed that spine growth induced under magnesium-free conditions actually does require protein synthesis, but only more than 30 minutes after LTP induction. This study also shows that the gradual recruitment of the postsynaptic scaffolding protein Homer Ib was abolished when protein synthesis was inhibited (Bosch et al., 20l4). Another study also showed that protein synthesis is involved in the maintenance of enlarged spines after LTP induction. Govindarajan and colleagues showed that spine growth returns to baseline after 2 hours, but spine growth could be maintained by pharmacological activation of PKA in the entire slice. This maintenance depended on protein synthesis. When glutamate uncaging was paired with PKA activation in the absence of protein synthesis, spine growth was entirely prevented (Govindarajan et al., 20ll). These studies illustrate that protein synthesis may be important for spine growth and functional LTP at the single synapse level under certain circumstances, but it is not clear how exactly it is triggered and when it is required.

\section{Brain Derived Neurotrophic Factor (BDNF) signaling}

The neurotrophic factor BDNF has been shown to affect single synapse LTP. Tanaka and colleagues suggested that BDNF is released after pairing glutamate uncaging with postsynaptic spiking, but not after glutamate uncaging in magnesium-free conditions (Tanaka et al., 2008). However, Harward and colleagues observed that a similar uncaging protocol in magnesiumfree conditions does lead to rapid release of BDNF from the stimulated spine, and that this is partially dependent on CaMKII activation. BDNF release resulted in rapid and sustained activation of the BDNF receptor TrkB in the stimulated spine, the dendrite and neighboring spines (Harward et al., 20I6). BDNF, via TrkB activation, may promote small GTPase and PKC activation (Colgan et al., 2018; Hedrick et al., 2016). In the Tanaka study, LTP was shown to require protein synthesis, while in the Harward study spine growth was independent of protein synthesis. These studies and others (Bosch et al., 2014) suggest that (autocrine) BDNF signaling can facilitate, but is not absolutely required for, single synapse LTP.They also show that subtle differences in stimulation protocol may lead to remarkable differences and illustrate our limited understanding of under which conditions BDNF is released from dendrites and spines.

\section{Spine shrinkage}

While we focus here on potentiation of spines, glutamate uncaging has also been used to 
induce shrinkage of spines and depression of synaptic transmission. Low frequency uncaging at a single spine ( 90 pulses at $0.1 \mathrm{~Hz}$, paired with depolarization) can induce spine shrinkage, which is accompanied by a decrease in uEPSC amplitude. The shrunken spines can undergo LTP and grow again when exposed to an LTP stimulus. There is an interesting difference between small and large spines: while large spines require metabotropic glutamate receptor (mGluR) and IP3 receptor activation to shrink, the small spines do not (Oh et al., 20I3). Spine shrinkage is dependent on non-ionotropic signaling of NMDA receptors, as it can occur without calcium flux through NMDA receptor channels. Surprisingly, a stimulation protocol that normally induces single synapse LTP leads to spine shrinkage when NMDA receptor-dependent calcium flow is inhibited, revealing that NMDA receptors may activate both pathways in parallel (Stein et al., 20I5). We refer interested readers to a more elaborate discussion of the molecular mechanisms involved in spine shrinkage and elimination (Stein and Zito, 2018).

\section{Multiple parallel pathways}

Studies on the induction of LTP in individual dendritic spines have revealed the temporal and spatial activation patterns of signaling molecules and pathways during LTP induction and expression. Single synapse LTP involves several, partially overlapping, intracellular signaling pathways, and the time course and magnitude of single synapse LTP is critically shaped by the molecular pathways involved. It will be important to gain a better understanding into the stimuli that trigger the different signaling pathways, and how multiple pathways interact within single spines and their direct vicinity.

The majority of studies use a magnesium-free protocol to assure NMDA receptor activation during the stimulation protocol and signaling pathways with this protocol have been described in great detail (Nakahata and Yasuda, 20I8; Nishiyama and Yasuda, 20I5). Under physiological conditions, glutamate receptor activation coincides with postsynaptic depolarization during LTP induction, which likely affects the spatial and temporal dynamics of signaling molecules in the stimulated spine and adjacent dendrite. Indeed, in a direct comparison, very different patterns of CaMKII activation were observed in magnesium-free and paired protocols (Lee et al., 2009). In addition, the requirements for protein synthesis and the contribution of BDNF signaling were found to be highly protocol-dependent (Govindarajan et al., 20 I ; Tanaka et al., 2008). This supports the idea that the spatiotemporal activation patterns of downstream signaling pathways are inevitably shaped by the induction protocol. This is important to realize, as experimental conditions are never fully representative of the in vivo physiological conditions. To interpret the intricate signaling pathways in the proper context, it is key to improve our understanding of how and when they are evoked at the single synapse level in vivo. 


\section{Interactions between synapses}

In the previous section we discussed the signaling pathways that can be activated when a single synapse undergoes LTP. Dendrites are tightly packed with hundreds of dendritic spines, and neighboring spines may influence each other. Under physiological conditions, single synapse activation may be rare and multiple synapses are receiving inputs simultaneously. It is therefore important to consider how adjacent synapses can influence each other's plasticity.

\section{Crosstalk}

Harvey \& Svoboda were the first to use glutamate uncaging to show crosstalk can occur between single spines during LTP induction: spines that received a weak ("subthreshold", I ms uncaging pulse) stimulus did not undergo LTP, but they only showed LTP when a nearby spine was stimulated with a strong (4 ms uncaging pulse) LTP-inducing stimulus. It is not clear whether the difference in pulse duration reflects a difference in the level and/or duration of NMDA receptor activation, or a difference in the type of glutamate receptors that are activated. The spine that received the weak stimulus showed the same level of spine growth and functional LTP as the spine that received the strong stimulus (Figure 2A). This crosstalk occurs over a timescale of several minutes and a length scale of 5-10 $\mu \mathrm{m}$, both in magnesiumfree and paired protocols (Harvey and Svoboda, 2007).

Several signaling molecules that are activated during LTP induction can diffuse out of the stimulated spine and affect signaling in neighboring spines. While calcium influx and CaMKII activation are brief and mostly restricted to the dendritic spine (when using a magnesiumfree induction protocol) (Harvey et al., 2008; Lee et al., 2009; Otmakhov et al., 20I5), their downstream effectors are often active for longer time scales and spread over longer distances. This has been studied mostly for the GTPases. After single synapse LTP induction, the GTPases Ras and Racl diffuse freely over approximately 10 micrometer within the dendrite and neighboring spines, while RhoA reaches $\sim 5 \mu$ (Harvey et al., 2008; Hedrick et al., 20 I6; Murakoshi et al., 20I I). Although Cdc42 is equally mobile as its family members, Cdc42 activation is contained within the spine head (Murakoshi et al., 20I I).

Diffusion of these signaling molecules can reduce the threshold for LTP in neighboring spines and thereby mediate synaptic crosstalk. When Ras signaling is pharmacologically inhibited, crosstalk is reduced (Harvey et al., 2008). Similarly, interfering with the spread of Racl and RhoA activity out of suprathreshold spine significantly reduces crosstalk without affecting the growth of the suprathreshold spine (Hedrick et al., 2016).A subthreshold stimulus (using shorter glutamate pulses) does not activate Ras, and only weakly activates Racl and RhoA.A suprathreshold stimulus on a spine nearby can elevate Ras, Racl and RhoA activation levels in the subthreshold spine above threshold. Cdc42 activation is similar after subthreshold and suprathreshold stimuli (Harvey et al., 2008; Hedrick et al., 2016).

During single synapse LTP, activation of PKC is almost completely restricted to the stimulated dendritic spine. However, when a nearby spine receives a subthreshold stimulus at the same time, PKC also gets activated in the subthreshold spine. PKC activation is triggered by fast and local calcium influx through NMDA receptors but is also sensitive to DAG production through TrkB-PLC signaling (Colgan et al., 2018). Because TrkB activation slowly spreads over a stretch of $10 \mu \mathrm{m}$ (Harward et al., 2016), PKC may integrate the activation history of nearby spines (Colgan et al., 2018). 


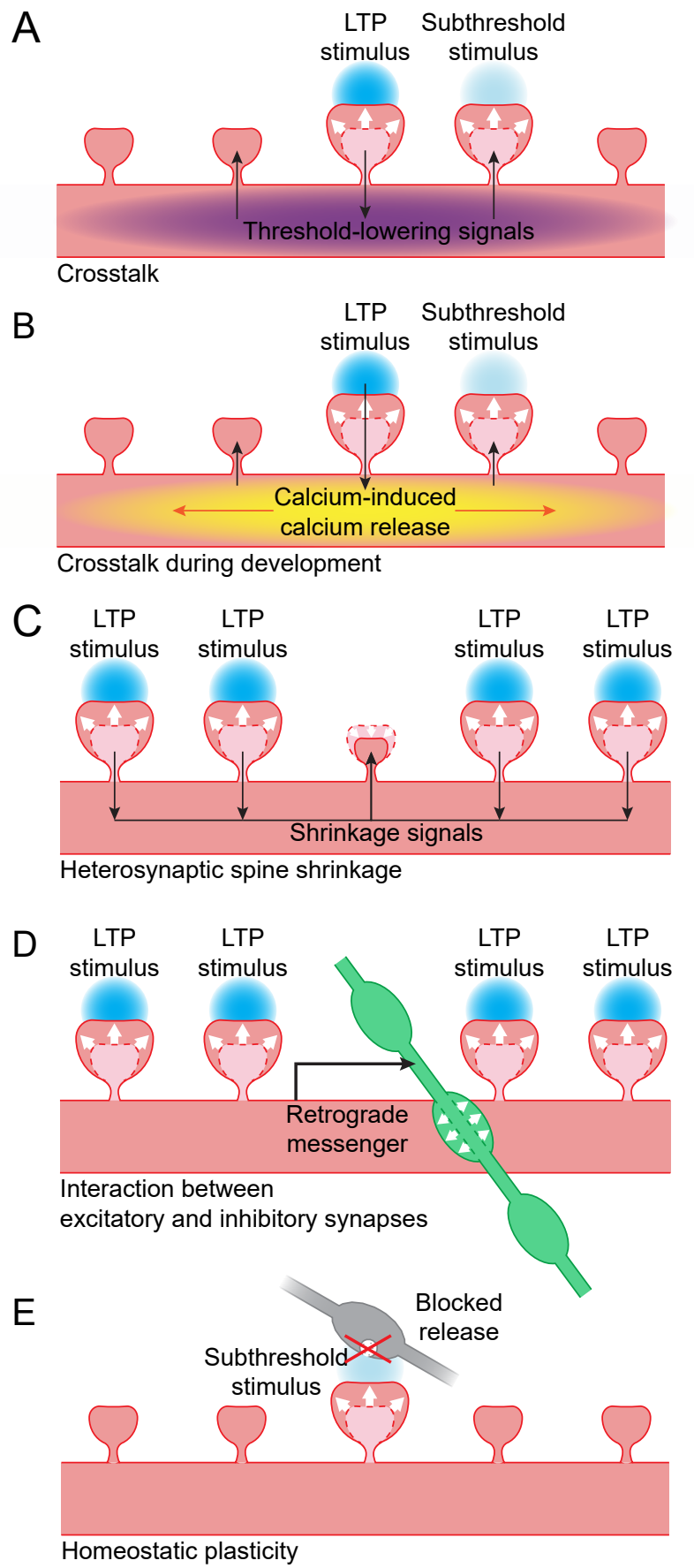

Figure 2. Interactions between synapses

A: When LTP is induced in a single spine using glutamate uncaging (blue), this leads to the spread of thresholdlowering signals (purple) in the dendrite. When a nearby spine receives a stimulus that is normally subthreshold, spine growth will occur.Threshold-lowering signals include the small GTPases Ras, Racl and RhoA (Harvey et al., 2008; Hedrick et al., 20I6; Murakoshi et al., 20II) and BDNF-TrkB signaling (Colgan et al., 20I8; Harward et al., 
2016). PKA and ERK activity also spreads over the dendrite but it is unclear if these kinases are able to lower the LTP threshold (Zhai et al., 20I3).

B: During development, the calcium influx in a single spine during glutamate uncaging can trigger calcium-induced calcium release (yellow). This leads to propagating calcium waves in the dendrite, and a nearby spine receiving a stimulus that is normally subthreshold will now show spine growth (Lee et al., 20I6).

C: When a cluster of spines undergo LTP, this can lead to the activation of shrinkage signals. These signals can induce shrinkage of a unstimulated dendritic spine nearby (Oh et al., 20I5).

D: When a cluster of spines undergo LTP, this can lead to the production of a retrograde messenger by the postsynaptic neuron. This messenger can trigger the growth of a presynaptic inhibitory bouton (green) nearby (Hu et al., 2019).

E: When vesicle fusion in the presynapse (gray) has been blocked for a prolonged period of time, this can lead to a lowering of the LTP threshold: when a spine receives a stimulus that is normally subthreshold, it will show spine growth (Lee et al., 20I0).

Both PKA and ERK activation spreads over more than $10 \mu \mathrm{m}$ of dendrite and invades nearby spines, with PKA showing a sharper spatial gradient and a more rapid decay than ERK. (Tang and Yasuda, 2017). ERK can stay active for a long time and diffuse over long distances within the dendritic tree. LTP induction on at least 3 spines on two different branches within 30 minutes leads to sustained nuclear ERK activation that is likely mediated by diffusion of activated ERK from the stimulated spines. Nuclear ERK activation is dependent on mGluR activation and may require PKC to maintain ERK activation. In the nucleus, ERK likely activates transcription factors that are responsible for the late phase of LTP (Zhai et al., 2013).

While the crosstalk described above typically works on a time scale of a few minutes (Harvey and Svoboda, 2007), another form of crosstalk has been observed on longer time scales. In the study by Govindarajan and colleagues, glutamate uncaging alone leads to spine growth that returns to baseline after 2 hours, while combining glutamate uncaging with pharmacological PKA activation leads to protein synthesis-dependent LTP that lasts for at least 4 hours. Interestingly, when a spine is exposed to glutamate uncaging alone before or after a neighboring spine is exposed to glutamate uncaging paired with PKA activation, both spines grow persistently for up to 4 hours. This crosstalk works over a time range of tens of minutes (both pre and post) and tens of micrometers on the same dendritic branch, and depends on protein synthesis (Govindarajan et al., 20l I).

Besides biochemical interactions, nearby spines will also interact electrically. Their postsynaptic potentials summate, often in nonlinear ways (London and Häusser, 2005; Losonczy and Magee, 2006; Tran-Van-Minh et al., 2015). For instance, it was shown that when four spines on a distal dendritic segment are stimulated, calcium levels in individual spines are higher than when the spines are activated individually and this is mediated by NMDA receptors. Simultaneous subthreshold stimulation at these spines (in the presence of magnesium and without depolarization), can overcome the LTP threshold and can induce functional LTP in these spines (Weber et al., 20I6).

Together, these studies show that single synapse LTP is affected by the recent activity of nearby spines and mediated by many factors, such as local kinase activity and dendritic exchange of GTPases (Nishiyama and Yasuda, 20I5; Yasuda, 20I7). Crosstalk of LTP between neighboring spines along the same dendrite is particularly relevant in vivo, where synapses with similar properties or activity patterns often clusters together (Bloss et al., 2016, 2018; lacaruso et al., 20 I7; Kleindienst et al., 20I I; Makino and Malinow, 20 I I;Wilson et al., 20 I6). 


\section{Plasticity and crosstalk during development}

During development, the rules for synaptic plasticity and crosstalk are not the same as in mature neurons (Lohmann and Kessels, 20l4). When uncaging at a single spine in young, developing neurons, calcium is less restricted in the spine head than in mature neurons, and calcium influx through NMDA channels can be boosted by calcium-induced calcium release (CICR) (Lee et al., 20I6). Activating individual spines often leads to propagating calcium waves in the dendrite that are mediated by CICR from intracellular stores. However, propagating calcium waves after LTP induction have not been observed in more mature neurons, suggesting that the coupling between NMDA receptors and internal calcium stores is developmentally regulated. In young neurons, all spine growth depends on calcium-induced calcium release, suggesting that calcium influx through NMDA receptors is not sufficient to induce LTP in young neurons. When a strong stimulus on one spine is paired with a weaker stimulus on a neighboring spine, this leads to sustained spine growth in both spines and this crosstalk is also dependent on CICR (Figure 2B). In general, the high level of local crosstalk in young neurons suggests the clustered maturation of spines. Indeed, it was shown that mature synapses, which have high AMPA/NMDA ratios, tend to cluster together on dendrites of young neurons (Lee et al., 20I6).

\section{Heterosynaptic spine shrinkage}

When a small cluster of spines (at least four) is potentiated using glutamate uncaging, it can induce shrinkage and loss of AMPA receptors at an unstimulated spine close to that cluster (Figure 2C) (Oh et al., 20I5). This heterosynaptic shrinkage is dependent on the calcium sensing protein calcineurin, mGluR and IP3 receptor signaling, but it is independent of the classical LTP protein CaMKII. When CaMKII is inhibited, spine growth at the stimulated spines is prevented, but the unstimulated spine still shrinks. When calcineurin is inhibited, only growth of the stimulated spines remains. This shows that the spine is not shrinking because of competition for resources, but because it is actively being regulated (Oh et al., 2015).

Spine shrinkage can also be induced by combining single spine glutamate uncaging with activation of dendritic $\mathrm{GABA}_{\mathrm{A}}$ receptors (Hayama et al., 2013). Neighboring spines within I5 $\mu \mathrm{m}$ also undergo shrinkage and synaptic transmission is weakened. This type of spine shrinkage depends on NMDA receptor and calcineurin signaling, but is independent of $m$ GluR signaling. While shrinkage spreads over the dendrite, a neighboring spine receiving a potentiating stimulus can still overcome the shrinkage signals and grow (Hayama et al., 20 I3). Together, these studies show that parallel signaling pathways for spine growth and shrinkage exist within the dendrite.

\section{Interaction between excitatory and inhibitory synapses}

Inhibitory synapses are important regulators of dendritic signals. They interact with excitatory synaptic inputs electrically, and they play an important role in regulating calcium dynamics in the dendrite (Higley, 20I4). An individual inhibitory synapse can reduce the influx of calcium during a backpropagating action potential locally within the dendrite (Müllner et al., 20I5) or even within a single spine (Chiu et al., 20/3). Additionally, activation of metabotropic $\mathrm{GABA}_{\mathrm{B}}$-receptors reduces NMDA receptor-mediated calcium influx in single activated spines (Chalifoux and Carter, 20I0). Inhibitory synapses are therefore likely able to interfere 
with nearby single synapse LTP induction. It needs to be noted that most studies discussed in this review use $\mathrm{MNI}$-glutamate as their caged compound, which has been shown to have strong antagonistic effects on GABA $_{A}$ receptors (Ellis-Davies, 2019; Fino et al., 2009; Matsuzaki et al., 2010). In addition, the presence of TTX in experiments using magnesiumfree induction protocols also abolishes spontaneous activity in inhibitory neurons. Inhibitory synaptic signaling might therefore be largely blocked in these studies, which may affect the induction and/or expression of single synapse LTP.

Vice versa, LTP at spines also affects nearby inhibitory synapses. Chemical and electrical LTP studies have shown that NMDA receptor activation affects gephyrin clusters and the surface expression of $\mathrm{GABA}_{\mathrm{A}}$ receptors (Flores et al., 20I5; Marsden et al., 2007; Petrini et al., 20I4) and leads to strengthening of inhibitory inputs (Bourne and Harris, 20I I; Chiu et al., 20I8). Using glutamate uncaging, our lab has recently shown that activation of a cluster of excitatory synapses can trigger the growth of a new inhibitory presynaptic bouton onto the stimulated dendrite via NMDA receptors and a retrograde endocannabinoid signal (Figure 2D) (Hu et al., 2019). Such a local coordination mechanism between excitatory and inhibitory plasticity will be important in regulating a balance between excitatory and inhibitory synapses within a dendritic branch, and ensuring local inhibitory control over an active excitatory cluster.

\section{Interaction with homeostatic plasticity}

Homeostatic plasticity operates over long time scales to maintain neuronal network function (Turrigiano, 2012). Neurons can regulate their own excitability by different mechanisms, including synaptic scaling of AMPA receptors (Turrigiano et al., 1998). Although the intracellular signaling pathways underlying synaptic scaling are not entirely clear, it is not unlikely that they partially overlap, or even interfere, with single synapse LTP. Lee and colleagues performed single synapse LTP at spines with silent (e.g. tetanus toxin expressing) presynaptic terminals, which had undergone synaptic scaling (Lee et al., 2010). They showed that presynaptic silencing leads to a decrease in LTP threshold, such that a stimulus protocol that is normally subthreshold can induce spine growth and functional LTP at presynaptically silenced spines (Figure 2E). They did not observe a difference in LTP when a suprathreshold stimulus was used (Lee et al., 2010). This suggests that homeostatic plasticity at individual synapses can affect the threshold for inducing spine growth and LTP.

Similarly, a recent study by Hobbiss and colleagues shows that when action potentials are blocked in a hippocampal slice for 48 hours using TTX, spines become bigger and stronger, indicative of synaptic scaling (Hobbiss et al., 2018). Using glutamate uncaging, the authors showed that small spines that were exposed to TTX treatment grow more after an LTP stimulus than untreated spines of the same size. In addition, a weak stimulus that does not induce sustained spine growth under control conditions, induces significant spine growth in the TTX condition. This suggests that homeostatic scaling enhances the capacity to undergo LTP (Hobbiss et al., 2018). However, in an earlier study by Soares and colleagues, no differences were observed in uncaging-induced spine growth between control and TTXtreated conditions (Soares et al., 2017). 


\section{Concluding remarks}

Since the first study reported LTP of a single dendritic spine using glutamate uncaging (Matsuzaki et al., 2004), several protocols have been used to induce single synapse LTP: magnesium-free protocols that do not require electrical access to the postsynaptic neuron or paired protocols attempting to resemble physiological activation of the postsynaptic neuron. The expression of LTP in a single synapse is measured by quantifying the increase in uEPSC amplitude and/or in spine size, which are highly correlated with one another. Thanks to tremendous technological advances, signaling pathways involved in single synapse LTP are studied with spectacularly high spatial and temporal resolution. Remarkably, these studies at the single synapse level revealed that synapses do not necessarily operate individually. Specific signaling proteins leave the spine head and penetrate into the dendritic shaft and nearby spines, where they can reduce the threshold for LTP.This implies that the activation and plasticity history of the synapse itself, as well as the history of synapses in its direct dendritic vicinity, strongly influences its capacity to undergo plasticity.

While we gained significantly more insight into the mechanisms of LTP at the single synapse level over the past 15 years, several questions remain and new questions emerge. There is sufficient evidence to conclude that different induction protocols trigger different signaling pathways and lead to different "modes" or levels of LTP expression. Morphological changes (peak and plateau spine growth) and functional LTP (receptor insertion) are not always perfectly aligned and may be evoked via different molecular routes with different experimental induction protocols. It will be the next challenge to understand if these parallel LTP pathways matter under physiological circumstances.

Another major challenge for the field is to understand the systems that are in place to coordinate the multitude of synaptic inputs within the neuron. Synapses with similar properties tend to cluster together on the same dendritic branch (Bloss et al., 2016, 2018; Druckmann et al., 20I4; lacaruso et al., 20I7; Kleindienst et al., 20I I; Wilson et al., 2016). One could therefore argue that in vivo, LTP rarely happens at isolated synapses but perhaps more often at small clusters of co-active synapses. It is therefore important to understand how spines undergoing LTP can interact within dendrites. Several studies have now started to address the mechanisms behind different forms of crosstalk. Expanding these studies to larger clusters of synapses, and including excitatory as well as inhibitory synapses, will allow us to examine under which circumstances synapses cooperate and when they compete for resources.

Research has focused on LTP in single spines, but the current understanding of synaptic depression and shrinkage of dendritic spines is much more limited. Only two uncaging protocols are known to induce LTD in the stimulated spine and one of those also requires GABA uncaging (Hayama et al., 20I3; Oh et al., 20I3). Spine shrinkage and synaptic depression are not regulated by the inverse of LTP pathways, but involve specific signaling. It will be important for future research to further unravel the spatial and temporal profile of LTDassociated signals and to examine overlap and interaction with LTP pathways.

In recent years, caged GABA compounds became available for two-photon uncaging. While uncaging GABA has been used to identify and quantify the presence of GABA receptors (Chiu et al., 20I3; Kanemoto et al., 20II; Kantevari et al., 20I0; Kwon et al., 20I8; Villa et al., 2016) and to induce nascent excitatory or inhibitory synapses in young neurons (Oh et 
al., 2016), two-photon GABA uncaging has yet to enter the realm of synaptic plasticity. It would be interesting to use GABA uncaging to assess changes in the strength of individual inhibitory synapses. Coordination between excitation and inhibition, which is crucial for the proper functioning of neurons, is regulated at the synaptic level (Bloss et al., 2016; Chen et al., 2012, 20I5; Hu et al., 2019; Liu, 2004). We therefore expect that improving our understanding of the interaction of excitatory and inhibitory plasticity at the level of single synapses and dendrites, for example by combining two-photon uncaging of glutamate and GABA (Kantevari et al., 2010), will provide us with exciting new insights.

The dendritic branch can be considered the fundamental electrical and biochemical functional unit of the nervous system (Branco and Häusser, 2010; Govindarajan et al., 20I I; LovettBarron et al., 2012). Single synapse LTP studies are revealing that the molecular signaling pathways underlying single synapse LTP are not limited to the stimulated spine, but kinases, GTPases and other regulators can travel and interact with proteins in the dendrite and neighboring synapses. The precise effect of synaptic activation depends therefore on the activation and plasticity history of the involved synapse, as well as excitatory and inhibitory synapses in its direct vicinity. Therefore, synaptic plasticity should always be considered within the context of the local dendritic homeostasis.

\section{Acknowledgements}

The authors would like to thank Helmut Kessels for critically reading the manuscript. This research was supported by the Netherlands Organization for Scientific Research, as part of the research programme of the Foundation for Fundamental Research on Matter (FOM) (\#I5PR3178-I). 


\section{References}

Araki,Y., Zeng, M., Zhang, M., and Huganir, R. L. (20I5). Rapid Dispersion of SynGAP from Synaptic Spines Triggers AMPA Receptor Insertion and Spine Enlargement during LTP. Neuron 85, 173 I89. doi:I0.1016/j.neuron.2014.12.023.

Araya, R., Jiang, J., Eisenthal, K. B., and Yuste, R. (2006). The spine neck filters membrane potentials. Proc. Natl. Acad. Sci. 103, 1796I-I7966. doi:10.1073/ pnas.0608755I 03.

Araya, R., Vogels, T. P., and Yuste, R. (20/4). Activitydependent dendritic spine neck changes are correlated with synaptic strength. Proc. Natl. Acad. Sci. III, E2895-2904. doi:10.1073/ pnas. I321869|II.

Asrican, B., Lisman, J., and Otmakhov, N. (2007) Synaptic Strength of Individual Spines Correlates with Bound $\mathrm{Ca}^{2+}$-Calmodulin-Dependent Kinase II. J. Neurosci. 27, I4007-|40|I. doi:I0.1523/ jneurosci.3587-07.2007.

Béïque, J.-C., Lin, D., Kang, M.-G., Aizawa, H., Takamiya, K., and Huganir, R. L. (2006). Synapse-specific regulation of AMPA receptor function by PSD95. Proc. Natl. Acad. Sci. 103, 19535-19540. doi: I0.1073/pnas.0608492103.

Berry, K. P., and Nedivi, E. (20I7). Spine Dynamics: Are They All the Same? Neuron 96, 43-55. doi:10.1016/j.neuron.2017.08.008.

Bliss, T.V. P., and Collingridge, G. L. (20I3). Expression of NMDA receptor-dependent LTP in the hippocampus: bridging the divide. Mol. Brain 6 , I-I4. doi:I0.I I86/I756-6606-6-5.

Bliss, T. V. P., and Lømo, T. (1973). Long-lasting potentiation of synaptic transmission in the dentate area of the anaesthetized rabbit following stimulation of the perforant path. J. Physiol. 232, 331-356. doi:10.1 I I3/jphysiol. 1973. sp010273.

Blitzer, R. D., Connor, J. H., Brown, G. P., Wong, T., Shenolikar, S., lyengar, R., et al. (1998). Gating of CaMKII by cAMP-regulated protein phosphatase activity during LTP. Science 280, 1940-1942. doi:10.1 126/science.280.5371.1940.

Bloss, E. B., Cembrowski, M. S., Karsh, B., Colonell, J., Fetter, R. D., and Spruston, N. (20I6). Structured Dendritic Inhibition Supports Branch-Selective Integration in CAI Pyramidal Cells. Neuron 89, 1016-1030. doi:10.1016/j.neuron.2016.01.029.
Bloss, E. B., Cembrowski, M. S., Karsh, B., Colonell, J., Fetter, R. D., and Spruston, N. (2018). Single excitatory axons form clustered synapses onto CAI pyramidal cell dendrites. Nat. Neurosci. 21, 353-363. doi:I0.1038/s4|593-0|8-0084-6.

Boehm, J., Kang, M. G., Johnson, R. C., Esteban, J., Huganir, R. L., and Malinow, R. (2006). Synaptic Incorporation of AMPA Receptors during LTP Is Controlled by a PKC Phosphorylation Site on GluRI. Neuron 5I, 213-225. doi:I0.1016/j. neuron.2006.06.013.

Bosch, M., Castro, J., Saneyoshi, T., Matsuno, H., Sur, M., and Hayashi, Y. (20I4). Structural and Molecular Remodeling of Dendritic Spine Substructures during Long-Term Potentiation. Neuron 82, 444459. doi:10.1016/j.neuron.2014.03.021.

Bosch, M., and Hayashi, Y. (20I2). Structural plasticity of dendritic spines. Curr. Opin. Neurobiol. 22, 383388. doi:10.1016/j.conb.20II.09.002.

Bourne, J. N., and Harris, K. M. (20I I). Coordination of Size and Number of Excitatory and Inhibitory Synapses Results in a Balanced Structural Plasticity Along Mature Hippocampal CAI Dendrites During LTP. Hippocampus 21, 354-373. doi:10.1002/hipo.20768.

Bouvier, G., Larsen, R.S., Rodríguez-Moreno,A.,Paulsen, O., and Sjöström, P. J. (20I8). Towards resolving the presynaptic NMDA receptor debate. Curr. Opin. Neurobiol. 5I, I-7. doi:10.1016/j. conb.2017.12.020

Branco, T., and Häusser, M. (20I0). The single dendritic branch as a fundamental functional unit in the nervous system. Curr. Opin. Neurobiol. 20, 494502. doi:10.1016/j.conb.2010.07.009.

Buchs, P. A., and Muller, D. (1996). Induction of longterm potentiation is associated with major ultrastructural changes of activated synapses. Proc. Natl. Acad. Sci. 93, 8040-8045. doi: 10.1073/ pnas.93.15.8040.

Busetto, G., Higley, M. J., and Sabatini, B. L. (2008). Developmental presence and disappearance of postsynaptically silent synapses on dendritic spines of rat layer $2 / 3$ pyramidal neurons. J. Physiol. 586, I519-1527. doi:10.1II3/ jphysiol.2007.I49336.

Caroni, P., Donato, F., and Muller, D. (20/2). Structural plasticity upon learning: Regulation and functions. Nat. Rev. Neurosci. 13, 478-490. doi:10.1038/ nrn3258. 
Chalifoux, J. R., and Carter, A. G. (2010). GABA Receptors Modulate NMDA Receptor Calcium Signals in Dendritic Spines. Neuron 66, I0I-I I3. doi:10.1016/j.neuron.2010.03.012.

Chalifoux, J. R., and Carter, A. G. (20II). Glutamate spillover promotes the generation of NMDA spikes. J. Neurosci. 31, 16435-16446. doi: I0.1523/ JNEUROSCI.2777-I I.20I I.

Chang, J.-Y., Nakahata, Y., Hayano, Y., and Yasuda, R. (20/9). Mechanisms of $\mathrm{Ca}^{2+} /$ calmodulindependent kinase II activation in single dendritic spines. Nat. Commun. 10, I-12. doi:10.1038/ s4|467-019-10694-z.

Chang, J.-Y., Parra-Bueno, P., Laviv, T., Szatmari, E. M., Lee, S.-J. R., and Yasuda, R. (2017). CaMKII Autophosphorylation Is Necessary for Optimal Integration of $\mathrm{Ca}^{2+}$ Signals during LTP Induction, but Not Maintenance. Neuron 94, 800-808. doi:I0.1016/j.neuron.2017.04.04I.

Chen, J. L., Villa, K. L., Cha, J. W., So, P. T. C., Kubota, Y., and Nedivi, E. (20/2). Clustered Dynamics of Inhibitory Synapses and Dendritic Spines in the Adult Neocortex. Neuron 74, 36I-373. doi:I0.1016/j.neuron.20I2.02.030.

Chen, S. X., Kim, A. N., Peters, A. J., and Komiyama, T. (20I5). Subtype-specific plasticity of inhibitory circuits in motor cortex during motor learning. Nat. Neurosci. 18, II09-III5. doi:10.1038/ nn.4049.

Chiu, C. Q., Lur, G., Morse, T. M., Carnevale, N. T., Ellis-Davies, G. C. R., and Higley, M. J. (20I3). Compartmentalization of GABAergic Inhibition by Dendritic Spines. Science 340, 759-762. doi: I0.I | 26/science. I 234274 .

Chiu, C. Q., Martenson, J. S., Yamazaki, M., Natsume, R., Sakimura, K., Tomita, S., et al. (20I8). InputSpecific NMDAR-Dependent Potentiation of Dendritic GABAergic Inhibition. Neuron 97, 368377. doi:10.1016/j.neuron.2017.12.032.

Chiu, S. L., Diering, G. H.,Ye, B.,Takamiya, K., Chen, C. M., Jiang,Y., et al. (2017). GRASPI Regulates Synaptic Plasticity and Learning through Endosomal Recycling of AMPA Receptors. Neuron 93, I405I4|9. doi:10.1016/j.neuron.2017.02.031.

Choquet, D. (2018). Linking Nanoscale Dynamics of AMPA Receptor Organization to Plasticity of Excitatory Synapses and Learning. J. Neurosci. 38, 93|8-9329. doi:10.1523/jneurosci.21 |9-18.2018.

Citri, A., and Malenka, R. C. (2008). Synaptic Plasticity:
Multiple Forms, Functions, and Mechanisms. Neuropsychopharmacology 33, I8-41. doi:10.1038/ sj.npp. I30I559.

Colgan, L.A., Hu, M., Misler, J.A., Parra-Bueno, P., Moran, C. M., Leitges, M., et al. (20I8). PKC $\alpha$ integrates spatiotemporally distinct $\mathrm{Ca}^{2+}$ and autocrine BDNF signaling to facilitate synaptic plasticity. Nat. Neurosci. 21, 1027-1037. doi:10.1038/ s41593-018-0184-3.

de Baaij, J. H. F., Hoenderop, J. G. J., and Bindels, R. J. M. (20/5). Magnesium in Man: Implications for Health and Disease. Physiol. Rev. 95, I-46. doi:10.1152/physrev.00012.2014.

De Roo, M., Klauser, P., and Muller, D. (2008). LTP promotes a selective long-term stabilization and clustering of dendritic spines. PLoS Biol. 6, I850|860. doi:I0.|37|/journal.pbio.0060219.

Denk, W., Strickler, J. H., and Webb, W.W. (1990). Twophoton laser scanning fluorescence microscopy. Science 248, 73-76. doi: I0.I I 26/science.2321 027.

Denk, W., and Svoboda, K. (1997). Photon Upmanship: Why Multiphoton Imaging is More than a Gimmick. Neuron 18, 35I-357. doi:10.1016/ s0896-6273(00)8I237-4.

Diering, G. H., and Huganir, R. L. (20/8). The AMPA Receptor Code of Synaptic Plasticity. Neuron I00, 314-329. doi:10.1016/j.neuron.2018.10.018.

Druckmann, S., Feng, L., Lee, B., Yook, C., Zhao, T., Magee, J. C., et al. (20|4). Structured Synaptic Connectivity between Hippocampal Regions. Neuron 81, 629-640. doi:10.1016/j. neuron.2013.11.026.

Ellis-Davies, G. C. R. (2019). Two-Photon Uncaging of Glutamate. Front. Synaptic Neurosci. 10, 1-13. doi:I0.3389/fnsyn.20I8.00048.

Esteban,J.A., Shi, S.-H.,Wilson, C., Nuriya, M., Huganir, R. L., and Malinow, R. (2003). PKA phosphorylation of AMPA receptor subunits controls synaptic trafficking underlying plasticity. Nat. Neurosci. 6, |36-|43. doi:10.1038/nn997.

Fino, E.,Araya, R., Peterka, D. S., Salierno, M., Etchenique, R., and Yuste, R. (2009). RuBi-Glutamate: Twophoton and visible-light photoactivation of neurons and dendritic spines. Front. Neural Circuits 3, I-9. doi:10.3389/neuro.04.002.2009.

Flores, C. E., Nikonenko, I., Mendez, P., Fritschy, J.-M., Tyagarajan, S. K., and Muller, D. (20I5). Activitydependent inhibitory synapse remodeling 
through gephyrin phosphorylation. Proc. Natl.Acad. Sci. I I2, E65-72. doi: 10.1073/pnas. I4 I I I70 I I2.

Fujii, H., Inoue, M., Okuno, H., Sano, Y., TakemotoKimura, S., Kitamura, K., et al. (20I3). Nonlinear Decoding and Asymmetric Representation of Neuronal Input Information by CaMKIll $\alpha$ and Calcineurin. Cell Rep. 3, 978-987. doi:10.1016/j. celrep.2013.03.033.

Gambino, F., Pagès, S., Kehayas, V., Baptista, D., Tatti, R., Carleton, A., et al. (20I4). Sensory-evoked LTP driven by dendritic plateau potentials in vivo. Nature 515, I I6-I 19. doi:I0.1038/nature I 3664.

Govindarajan, A., Israely, I., Huang, S.Y., and Tonegawa, S. (20II). The Dendritic Branch Is the Preferred Integrative Unit for Protein Synthesis-Dependent LTP. Neuron 69, 132-146. doi:10.1016/j. neuron.2010.12.008.

Hamilton, A. M., Oh, W. C., Vega-Ramirez, H., Stein, I. S., Hell, J.W., Patrick, G. N., et al. (20I2). ActivityDependent Growth of New Dendritic Spines Is Regulated by the Proteasome. Neuron 74, 1023 1030. doi:10.1016/j.neuron.2012.04.031.

Harvey, C. D., and Svoboda, K. (2007). Locally dynamic synaptic learning rules in pyramidal neuron dendrites. Nature 450, I 195-1200. doi:10.1038/ nature 06416 .

Harvey, C. D., Yasuda, R., Zhong, H., and Svoboda, K. (2008). The Spread of Ras Activity Triggered by Activation of a Single Dendritic Spine. Science 32 I, |36-|40. doi:I0.I |26/science. I I59675.

Harward, S. C., Hedrick, N. G., Hall, C. E., Parrabueno, P., Milner, T. A., Pan, E., et al. (20I6). Autocrine BDNF-TrkB signalling within a single dendritic spine. Nature 538,99-103. doi:10.1038/ naturel 9766.

Hayama, T., Noguchi, J., Watanabe, S., Takahashi, N., Hayashi-Takagi, A., Ellis-Davies, G. C. R., et al. (20I3). GABA promotes the competitive selection of dendritic spines by controlling local $\mathrm{Ca}^{2+}$ signaling. Nat. Neurosci. 16, 1409-1416. doi: 10.1038/nn.3496.

Hayashi-Takagi, A., Yagishita, S., Nakamura, M., Shirai, F., Wu, Y. I., Loshbaugh, A. L., et al. (20I5). Labelling and optical erasure of synaptic memory traces in the motor cortex. Nature 525, 333-338. doi:10.1038/nature 15257 .

Hedrick, N. G., Harward, S. C., Hall, C. E., Murakoshi, H., McNamara, J. O., and Yasuda, R. (20I6). Rho GTPase complementation underlies BDNF- dependent homo- and heterosynaptic plasticity. Nature 538, 104-108. doi:10.1038/nature 9784.

Hell, J.W. (20|4). CaMKII: Claiming Center Stage in Postsynaptic Function and Organization. Neuron 8I, 249-265. doi:10.1016/j.neuron.2013.12.024.

Helmchen, F., and Denk, W. (2005). Deep tissue twophoton microscopy. Nat. Methods 2, 932-940. doi:I0.1038/nmeth8I8.

Herring, B. E., and Nicoll, R. A. (2016). Long-Term Potentiation: From CaMKII to AMPA Receptor Trafficking. Annu. Rev. Physiol. 78, 35I-365. doi:I0.I I46/annurev-physiol-02 I0I4-07I753.

Higley, M. J. (20I4). Localized GABAergic inhibition of dendritic $\mathrm{Ca}^{2+}$ signalling. Nat. Rev. Neurosci. I5, 567-572. doi: 10.1038/nrn3803.

Hill, T. C., and Zito, K. (20I3). LTP-Induced Long-Term Stabilization of Individual Nascent Dendritic Spines. J. Neurosci. 33, 678-686. doi:10.1523/ JNEUROSCI. I404-12.20I3.

Hobbiss, A. F., Ramiro-Cortés, Y., and Israely, I. (20I8). Homeostatic Plasticity Scales Dendritic Spine Volumes and Changes the Threshold and Specificity of Hebbian Plasticity. iScience 8, 16I174. doi:10.1016/j.isci.2018.09.015.

Hofer, S. B., Mrsic-Flogel, T. D., Bonhoeffer, T., and Hübener, M. (2009). Experience leaves a lasting structural trace in cortical circuits. Nature 457, 3|3-3|7. doi:10.1038/nature07487.

Holtmaat,A.,Wilbrecht, L., Knott, G.W.,Welker, E., and Svoboda, K. (2006). Experience-dependent and cell-type-specific spine growth in the neocortex. Nature 44I, 979-983. doi:I0.1038/nature04783.

Honkura, N., Matsuzaki, M., Noguchi, J., Ellis-Davies, G. C. R., and Kasai, H. (2008). The Subspine Organization of Actin Fibers Regulates the Structure and Plasticity of Dendritic Spines. Neuron 57, 719-729. doi:10.1016/j. neuron.2008.01.013.

Hotulainen, P., and Hoogenraad, C. C. (20I0). Actin in dendritic spines: Connecting dynamics to function. J. Cell Biol. 189, 619-629. doi:10.1083/ jcb.201003008.

Hu, H. Y., Kruijssen, D. L. H., Frias, C. P., Rózsa, B., Hoogenraad, C. C., and Wierenga, C. J. (2019). Endocannabinoid Signaling Mediates Local Dendritic Coordination between Excitatory and Inhibitory Synapses. Cell Rep. 27, 666-675. doi:10.1016/j.celrep.2019.03.078. 
Huganir, R. L., and Nicoll, R. A. (2013). AMPARs and Synaptic Plasticity: The Last 25 Years. Neuron 80, 704-717. doi:10.1016/j.neuron.2013.10.025.

lacaruso, M. F., Gasler, I. T., and Hofer, S. B. (20I7). Synaptic organization of visual space in primary visual cortex. Nature 547, 449-452. doi: 10.1038/ nature23019.

Incontro, S., Díaz-Alonso, J., lafrati, J.,Vieira, M.,Asensio, C. S., Sohal, V. S., et al. (20I8). The CaMKII/ NMDA receptor complex controls hippocampal synaptic transmission by kinase-dependent and independent mechanisms. Nat. Commun. 9, I-2I. doi:10.1038/s4|467-018-04439-7.

Kanemoto, Y., Matsuzaki, M., Morita, S., Hayama, T., Noguchi, J., Senda, N., et al. (20II). Spatial Distributions of GABA Receptors and Local Inhibition of $\mathrm{Ca}^{2+}$ Transients Studied with GABA Uncaging in the Dendrites of CAI Pyramidal Neurons. PLoS One 6, I-6. doi:I0.137//journal. pone.0022652.

Kantevari, S., Matsuzaki, M., Kanemoto, Y., Kasai, H., and Ellis-Davies, G. C. R. (20I0). Two-color, twophoton uncaging of glutamate and GABA. Nat. Methods 7, I23-125. doi:10.1038/nmeth.14I3.

Kessels, H.W., and Malinow, R. (2009). Synaptic AMPA Receptor Plasticity and Behavior. Neuron 6I, 340-350. doi:10.1016/j.neuron.2009.01.015.

Kim, K., Lakhanpal, G., Lu, H. E., Khan, M., Suzuki, A., Hayashi, M. K., et al. (20I5). A Temporary Gating of Actin Remodeling during Synaptic Plasticity Consists of the Interplay between the Kinase and Structural Functions of CaMKII. Neuron 87, 813-826. doi:10.1016/j.neuron.2015.07.023.

Kim, K., Suzuki, A., Kojima, H., Kawamura, M., Miya, K., Abe, M., et al. (2019). Autophosphorylation of $\mathrm{F}$-actin binding domain of $\mathrm{CaMKII} \beta$ is required for fear learning. Neurobiol. Learn. Mem. 157, 8695. doi:10.1016/j.nIm.2018.12.003.

Kleindienst, T., Winnubst, J., Roth-Alpermann, C., Bonhoeffer, T., and Lohmann, C. (20II). Activity-Dependent Clustering of Functional Synaptic Inputs on Developing Hippocampal Dendrites. Neuron 72, 10I2-1024. doi:10.1016/j. neuron.20II.I0.0I5.

Kwon, H.-B., and Sabatini, B. L. (20II). Glutamate induces de novo growth of functional spines in developing cortex. Nature 474, 100-104. doi: $10.1038 /$ nature09986.

Kwon, T., Merchán-Pérez, A., Rial Verde, E. M.,
Rodríguez, J.-R., DeFelipe, J., and Yuste, R. (20I8). Ultrastructural, Molecular and Functional Mapping of GABAergic Synapses on Dendritic Spines and Shafts of Neocortical Pyramidal Neurons. Cereb. Cortex, I-II. doi:10.1093/ cercor/bhyl 43 .

Lang, C., Barco,A., Zablow, L., Kandel, E. R., Siegelbaum, S. A., and Zakharenko, S. S. (2004). Transient expansion of synaptically connected dendritic spines upon induction of hippocampal long-term potentiation. Proc. Natl. Acad. Sci. I0I, I6665|6670. doi: 10.1073/pnas.040758I I0I.

Lee, K. F. H., Soares, C., Thivierge, J.-P., and Béïque, J.-C. (2016). Correlated Synaptic Inputs Drive Dendritic Calcium Amplification and Cooperative Plasticity during Clustered Synapse Development. Neuron 89, I-16. doi:10.1016/j. neuron.2016.01.012.

Lee, M.-C., Yasuda, R., and Ehlers, M. D. (20I0). Metaplasticity at Single Glutamatergic Synapses. Neuron 66, 859-870. doi:10.1016/j. neuron.2010.05.015.

Lee, S.-J. R., Escobedo-Lozoya, Y., Szatmari, E. M., and Yasuda, R. (2009). Activation of CaMKII in single dendritic spines during long-term potentiation. Nature 458, 299-304. doi:I0.1038/nature07842.

Lipp, P., and Reither, G. (20I I). Protein Kinase C: the "Masters" of Calcium and Lipid. Cold Spring Harb. Perspect. Biol. 3, I-I7. doi:I0.1 I0I/cshperspect. a004556.

Liu, G. (2004). Local structural balance and functional interaction of excitatory and inhibitory synapses in hippocampal dendrites. Nat. Neurosci. 7, 373379. doi: $10.1038 / \mathrm{nn} \mid 206$.

Lohmann, C., and Kessels, H. W. (20l4). The developmental stages of synaptic plasticity. J. Physiol. 592, 13-31. doi:I0.1II3/ jphysiol.2012.235119.

London, M., and Häusser, M. (2005). Dendritic computation. Annu. Rev. Neurosci. 28, 503-532. doi:I0.1 |46/annurev.neuro.28.06|604.135703.

Losonczy, A., and Magee, J. C. (2006). Integrative Properties of Radial Oblique Dendrites in Hippocampal CAI Pyramidal Neurons. Neuron 50, 29I-307. doi:10.1016/j.neuron.2006.03.016.

Lovett-Barron, M., Turi, G. F., Kaifosh, P., Lee, P. H., Bolze, F., Sun, X.-H., et al. (20I2). Regulation of neuronal input transformations by tunable dendritic inhibition. Nat. Neurosci. 15, 423-430. 
doi: $10.1038 / \mathrm{nn} .3024$.

Makino, H., and Malinow, R. (2009). AMPA Receptor Incorporation into Synapses during LTP:The Role of Lateral Movement and Exocytosis. Neuron 64, 38I-390. doi:I0.1016/j.neuron.2009.08.035.

Makino, H., and Malinow, R.(20I I). Compartmentalized versus Global Synaptic Plasticity on Dendrites Controlled by Experience. Neuron 72, 100II0II. doi:10.1016/j.neuron.20II.09.036.

Malenka, R. C., and Bear, M. F. (2004). LTP and LTD: An Embarrassment of Riches. Neuron 44, 5-2I. doi:I0.1016/j.neuron.2004.09.012.

Malinow, R., and Tsien, R. W. (1990). Presynaptic enhancement shown by whole-cell recordings of long-term potentiation in hippocampal slices. Nature 346, I77-I80. doi:I0.1038/346I77a0.

Man, H.-Y., Sekine-Aizawa, Y., and Huganir, R. L. (2007). Regulation of $\alpha$-amino-3-hydroxy-5-methyl4-isoxazolepropionic acid receptor trafficking through PKA phosphorylation of the Glu receptor I subunit. Proc. Natl.Acad. Sci. I04, 3579_ 3584. doi:10.1073/pnas.06II698I04.

Marsden, K. C., Beattie, J. B., Friedenthal, J., and Carroll, R. C. (2007). NMDA Receptor Activation Potentiates Inhibitory Transmission through GABA Receptor-Associated ProteinDependent Exocytosis of $\mathrm{GABA}_{\mathrm{A}}$ Receptors. J. Neurosci. 27, 14326-|4337. doi:10.1523/ JNEUROSCI.4433-07.2007.

Masters, B. R., and So, P. T. C. (2004). Antecedents of Two-Photon Excitation Laser Scanning Microscopy. Microsc. Res. Tech. 63, 3-II. doi:I0.1002/jemt. 10418.

Matsuzaki, M., Ellis-Davies, G. C. R., Nemoto, T., Miyashita, Y., lino, M., and Kasai, H. (200I). Dendritic spine geometry is critical for AMPA receptor expression in hippocampal CAI pyramidal neurons. Nat. Neurosci. 4, I086-1092. doi: $10.1038 / n n 736$.

Matsuzaki, M., Hayama, T., Kasai, H., and EllisDavies, G. C. R. (20I0). Two-photon uncaging of $\gamma$-aminobutyric acid in intact brain tissue. Nat. Chem. Biol. 6, 255-257. doi:10.1038/ nchembio.32I.

Matsuzaki, M., Honkura, N., Ellis-Davies, G. C. R., and Kasai, H. (2004). Structural basis of long-term potentiation in single dendritic spines. Nature 429, 76I-766. doi:10.1038/nature026I7.
Mayford, M., Siegelbaum, S.A., and Kandel, E. R. (20I2). Synapses and memory storage. Cold Spring Harb. Perspect. Biol. 4, I-I8. doi:I0.1 I01/cshperspect. a00575I.

Meyer, D., Bonhoeffer, T., and Scheuss, V. (20I4). Balance and Stability of Synaptic Structures during Synaptic Plasticity. Neuron 82, 430-443. doi:I0.1016/j.neuron.2014.02.03I.

Moretto, E., and Passafaro, M. (2018). Recent Findings on AMPA Receptor Recycling. Front. Cell. Neurosci. I2, I-I2. doi:I0.3389/fncel.2018.00286.

Müllner, F. E.,Wierenga, C. J., and Bonhoeffer, T. (20I5). Precision of Inhibition: Dendritic Inhibition by Individual GABAergic Synapses on Hippocampal Pyramidal Cells Is Confined in Space and Time. Neuron 87, 576-589. doi:10.1016/j. neuron.2015.07.003.

Murakoshi, H., Shin, M. E., Parra-Bueno, P., Szatmari, E. M., Shibata, A. C. E., and Yasuda, R. (2017). Kinetics of Endogenous CaMKII Required for Synaptic Plasticity Revealed by Optogenetic Kinase Inhibitor. Neuron 94, I-II. doi: 10.1016/j. neuron.2017.02.036.

Murakoshi, H., Wang, H., and Yasuda, R. (20II). Local, persistent activation of Rho GTPases during plasticity of single dendritic spines. Nature 472 , 100-106. doi:10.1038/nature09823.

Nabavi, S., Kessels, H. W., Alfonso, S., Aow, J., Fox, R., and Malinow, R. (20I3). Metabotropic NMDA receptor function is required for NMDA receptor-dependent long-term depression. Proc. Natl. Acad. Sci. II0, 4027-4032. doi:10.1073/ pnas. I219454II0.

Nakahata, Y., and Yasuda, R. (2018). Plasticity of Spine Structure: Local Signaling, Translation and Cytoskeletal Reorganization. Front. Synaptic Neurosci. 10, I-13. doi:10.3389/fnsyn.2018.00029.

Nicoll, R. A. (2017). A Brief History of Long-Term Potentiation. Neuron 93, 28I-290. doi: 10.1016/j. neuron.2016.12.015.

Nishiyama, J., and Yasuda, R. (20I5). Biochemical Computation for Spine Structural Plasticity. Neuron 87, 63-75. doi:10.1016/j. neuron.2015.05.043.

Noguchi, J., Hayama, T.,Watanabe, S., Ucar, H., Yagishita, S., Takahashi, N., et al. (20I6). State-dependent diffusion of actin-depolymerizing factor/cofilin underlies the enlargement and shrinkage of dendritic spines. Sci. Rep. 6, I-9. doi:I0.1038/ 
srep32897.

Noguchi, J., Matsuzaki, M., Ellis-Davies, G. C. R., and Kasai, H. (2005). Spine-Neck Geometry Determines NMDA Receptor-Dependent $\mathrm{Ca}^{2+}$ Signaling in Dendrites. Neuron 46, 609-622. doi:10.1016/j.neuron.2005.03.015.

Noguchi, J., Nagaoka, A., Watanabe, S., Ellis-Davies, G. C. R., Kitamura, K., Kano, M., et al. (20II). In vivo two-photon uncaging of glutamate revealing the structure-function relationships of dendritic spines in the neocortex of adult mice. J. Physiol. 589, 2447-2457. doi:I0.1II3/ jphysiol.2011.207100.

Oh, W. C., Hill, T. C., and Zito, K. (20I3). Synapsespecific and size-dependent mechanisms of spine structural plasticity accompanying synaptic weakening. Proc. Natl. Acad. Sci. I I0, E305-E3 I 2. doi:10.1073/pnas. I214705II0.

Oh, W. C., Lutzu, S., Castillo, P. E., and Kwon, H.-B. (20I6). De novo synaptogenesis induced by GABA in the developing mouse cortex. Science 353, 1037-1040. doi: 10.1 126/science.aaf5206.

Oh, W. C., Parajuli, L. K., and Zito, K. (20I5). Heterosynaptic Structural Plasticity on Local Dendritic Segments of Hippocampal CAI Neurons. Cell Rep. 10, 162-169. doi:10.1016/j. celrep.2014.12.016.

Okamoto, K.-I., Nagai, T., Miyawaki, A., and Hayashi, Y. (2004). Rapid and persistent modulation of actin dynamics regulates postsynaptic reorganization underlying bidirectional plasticity. Nat. Neurosci. 7, II04-III2. doi:I0.1038/nnI3II.

Otmakhov, N., Regmi, S., and Lisman, J. E. (20I5). Fast decay of CaMKII FRET sensor signal in spines after LTP induction is not due to its dephosphorylation. PLoS One 10, I-13. doi:10.137I/journal.pone.0130457.

Parsons, M. P., and Raymond, L.A. (20|4). Extrasynaptic NMDA Receptor Involvement in Central Nervous System Disorders. Neuron 82, 279-293. doi:I0.1016/j.neuron.2014.03.030.

Patterson, M. A., Szatmari, E. M., and Yasuda, R. (20I0). AMPA receptors are exocytosed in stimulated spines and adjacent dendrites in a Ras-ERK-dependent manner during long-term potentiation. Proc. Natl. Acad. Sci. 107, I595II5956. doi:10.1073/pnas.09|3875107.

Patterson, M., and Yasuda, R. (20II). Signalling pathways underlying structural plasticity of dendritic spines. Br.J. Pharmacol. 163, 1626-1638. doi:I0.1 I II/j. I476-538I.20II.01328.x.

Petrini,E.M., Ravasenga,T., Hausrat,T.J., lurilli, G., Olcese, U., Racine, V., et al. (20I4). Synaptic recruitment of gephyrin regulates surface $\mathrm{GABA}_{\mathrm{A}}$ receptor dynamics for the expression of inhibitory LTP. Nat. Commun. 5, I-I9. doi:I0.1038/ncomms492I.

Roberts, T. F., Tschida, K. A., Klein, M. E., and Mooney, R. (20I0). Rapid spine stabilization and synaptic enhancement at the onset of behavioural learning. Nature 463, 948-952. doi:10.1038/ nature08759.

Rusakov, D.A., and Kullmann, D.M. (1998). Extrasynaptic Glutamate Diffusion in the Hippocampus: Ultrastructural Constraints, Uptake, and Receptor Activation. J. Neurosci. 18, 3158-3170. doi:I0.1523/JNEUROSCI.I8-09-03।58.1998.

Saneyoshi, T., Matsuno, H., Suzuki, A., Murakoshi, H., Hedrick, N. G., Agnello, E., et al. (2019). Reciprocal Activation within a Kinase-Effector Complex Underlying Persistence of Structural LTP. Neuron 102, I199-1210. doi:10.1016/j. neuron.2019.04.012.

Scheefhals, N., and MacGillavry, H.D. (20I8). Functional organization of postsynaptic glutamate receptors. Mol. Cell. Neurosci. 91, 82-94. doi:10.1016/j. mcn.2018.05.002.

Sjöström, P. J., Rancz, E. A., Roth, A., and Häusser, M. (2008). Dendritic Excitability and Synaptic Plasticity. Physiol. Rev. 88, 769-840. doi:I0.II52/ physrev.00016.2007.

Soares, C., Lee, K. F. H., and Béïque, J. C. (20I7). Metaplasticity at CAI Synapses by Homeostatic Control of Presynaptic Release Dynamics. Cell Rep. 21, 1293-1303. doi:10.1016/j. celrep.2017.10.025.

Sobczyk, A., Scheuss, V., and Svoboda, K. (2005). NMDA Receptor Subunit-Dependent $\left[\mathrm{Ca}^{2+}\right]$ Signaling in Individual Hippocampal Dendritic Spines. J. Neurosci. 25, 6037-6046. doi:10.1523/ JNEUROSCI. I 22I-05.2005.

Soeller, C., and Cannell, M. B. (1999). Two-Photon Microscopy: Imaging in Scattering Samples and Three- Dimensionally Resolved Flash Photolysis. Microsc. Res. Tech. 47, 182-195. doi:10.1002/ (SICI) I097-0029(I999I। 0I) 47:3< I82::AIDJEMT4>3.0.CO;2-4.

Stein, I. S., Gray, J. A., and Zito, K. (20I5). Nonlonotropic NMDA Receptor Signaling Drives 
Activity-Induced Dendritic Spine Shrinkage. J. Neurosci. 35, 12303-12308. doi:10.1523/ JNEUROSCI.4289-I4.20I5.

Stein, I. S., and Zito, K. (20/8). Dendritic Spine Elimination: Molecular Mechanisms and Implications. Neurosci., 10738584/876964. doi: I0.1 I77/I0738584|8769644.

Steiner, P., Higley, M. J., Xu, W., Czervionke, B. L., Malenka, R. C., and Sabatini, B. L. (2008). Destabilization of the Postsynaptic Density by PSD-95 Serine 73 Phosphorylation Inhibits Spine Growth and Synaptic Plasticity. Neuron 60, 788802. doi:10.1016/j.neuron.2008.10.014.

Svoboda, K., and Yasuda, R. (2006). Principles of Two-Photon Excitation Microscopy and Its Applications to Neuroscience. Neuron 50, 823839. doi:10.1016/j.neuron.2006.05.019.

Tanaka, J.-I., Horiike, Y., Matsuzaki, M., Miyazaki, T., Ellis-Davies, G. C. R., and Kasai, H. (2008). Protein Synthesis and Neurotrophin-Dependent Structural Plasticity of Single Dendritic Spines. Science 319, 1683-1687. doi:10.1126/ science. II 52864

Tang, S., and Yasuda, R. (20I7). Imaging ERK and PKA Activation in Single Dendritic Spines during Structural Plasticity. Neuron 93, 13/5-1324. doi:10.1016/j.neuron.2017.02.032.

Tønnesen, J., Inavalli,V.V. G. K., and Nägerl, U.V. (20I8). Super-Resolution Imaging of the Extracellular Space in Living Brain Tissue. Cell I72, I I08-I I 2 I. doi:I0.1016/j.cell.20I8.02.007.

Tønnesen, J., Katona, G., Rózsa, B., and Nägerl, U. V. (20|4). Spine neck plasticity regulates compartmentalization of synapses. Nat. Neurosci. 17, 678-685. doi: I0.1038/nn.3682.

Tran-Van-Minh, A., Cazé, R. D., Abrahamsson, T., Cathala, L., Gutkin, B. S., and DiGregorio, D. A (20I5). Contribution of sublinear and supralinear dendritic integration to neuronal computations. Front. Cell. Neurosci. 9, I-15. doi:10.3389/ fncel.20I5.00067.

Turrigiano, G. (20/2). Homeostatic Synaptic Plasticity: Local and Global Mechanisms for Stabilizing Neuronal Function. Cold Spring Harb. Perspect. Biol. 4, I-I8. doi: I0. I I0 I/cshperspect.a005736.

Turrigiano, G. G., Leslie, K. R., Desai, N. S., Rutherford, L. C., and Nelson, S. B. (1998). Activitydependent scaling of quantal amplitude in neocortical neurons. Nature 391, 892-896. doi: $10.1038 / 36103$

Ueda,Y., Kwok, S., and Hayashi,Y. (20 I 3).Application of FRET probes in the analysis of neuronal plasticity. Front. Neural Circuits 7, I-19. doi:10.3389/ fncir.2013.00163.

Van Harreveld, A., and Fifkova, E. (1975). Swelling of dendritic spines in the fascia dentata after stimulation of the perforant fibers as a mechanism of post-tetanic potentiation. Exp. Neurol. 49, 736749. doi: I0.10I6/00 I4-4886(75)90055-2.

Villa, K. L., Berry, K. P., Subramanian, J., Cha, J.W., Oh,W. C., Kwon, H.-B., et al. (20I6). Inhibitory Synapses Are Repeatedly Assembled and Removed at Persistent Sites In Vivo. Neuron 89, 756-769. doi:10.1016/j.neuron.2016.01.010.

Weber, J. P., Andrásfalvy, B. K., Polito, M., Magó, Á., Ujfalussy, B. B., and Makara, J. K. (20I6). Locationdependent synaptic plasticity rules by dendritic spine cooperativity. Nat. Commun. 7, I-14. doi: I0.1038/ncomms I I 380 .

Wiegert, J. S., Pulin, M., Gee, C. E., and Oertner, T. G. (20I8). The fate of hippocampal synapses depends on the sequence of plasticity-inducing events. Elife 7, I-I8. doi:I0.7554/eLife.3915I.

Wilson, D. E., Whitney, D. E., Scholl, B., and Fitzpatrick, D. (20/6). Orientation selectivity and the functional clustering of synaptic inputs in primary visual cortex. Nat. Neurosci. 19, I0031009. doi: I0.1038/nn.4323.

Yagishita, S., Hayashi-Takagi, A., Ellis-Davies, G. C. R., Urakubo, H., Ishii, S., and Kasai, H. (20।4). A critical time window for dopamine actions on the structural plasticity of dendritic spines. Science 345, |6 |6-|620. doi: | 0. I | 26/science. I2555 | 4 .

Yasuda, R. (20I2). Studying Signal Transduction in Single Dendritic Spines. Cold Spring Harb. Perspect. Biol. 4, I-I5. doi: I0. I I0 I/cshperspect.a0056 I I.

Yasuda, R. (2017). Biophysics of Biochemical Signaling in Dendritic Spines: Implications in Synaptic Plasticity. Biophys. J., I-8. doi:10.1016/j. bpj.2017.07.029.

Zhai, S., Ark, E. D., Parra-bueno, P., and Yasuda, R. (20I3). Long-Distance Integration of Nuclear ERK signaling Triggered by Activation of a Few Dendritic Spines. Science 292, II07-IIII. doi: I0.1 | 26/science. 1245622

Zhang, Y.-P., Holbro, N., and Oertner, T. G. (2008). Optical induction of plasticity at single 
synapses reveals input-specific accumulation of aCaMKII. Proc. Natl. Acad. Sci. 105, I2039-1 2044. doi:I0.1073/pnas.0802940I05.

Zhou, Q., Homma, K. J., and Poo, M.-M. (2004). Shrinkage of Dendritic Spines Associated with Long-Term Depression of Hippocampal Synapses. Neuron 44, 749-757. doi:10.1016/j. neuron.2004.II.0II.

Zipfel, W. R., Williams, R. M., and Webb, W.W. (2003). Nonlinear magic: multiphoton microscopy in the biosciences. Nat. Biotechnol. 21, 1369-77. doi: $10.1038 /$ nbt899.

Zito, K., Scheuss, V., Knott, G., Hill, T., and Svoboda, K. (2009). Rapid Functional Maturation of Nascent Dendritic Spines. Neuron 6I, 247-258. doi:10.1016/j.neuron.2008.10.054. 


\section{Endocannabinoid signaling mediates local dendritic coordination between excitatory and inhibitory synapses}

Hai Yin Hu*1, Dennis L. H. Kruijssen*1, Cátia P. Frias',2, Balázs Rózsa ${ }^{3,4}$, Casper C. Hoogenraad', Corette J.Wierenga'

\footnotetext{
' Department of Biology, Science for Life, Utrecht University, Utrecht, The Netherlands

${ }^{2}$ Current address: Department of Bionanoscience, Kavli Institute of Nanoscience, Delft University of Technology, Delft, The Netherlands

${ }^{3}$ Laboratory of 3D Functional Network and Dendritic Imaging, Institute of Experimental Medicine, Hungarian Academy of Sciences, Budapest, Hungary

${ }^{4}$ Faculty of Information Technology, Pázmány Péter Catholic University, Budapest, Hungary

* These authors contributed equally
}

An adapted version of this chapter was published in Cell Reports 27 (2019). 


\section{Abstract}

Dendritic inhibitory synapses are most efficient in modulating excitatory inputs localized on the same dendrite, but it is unknown if their location is random or regulated. Here we show that formation of inhibitory synapses can be directed by excitatory synaptic activity on the same dendrite. We stimulated dendritic spines close to a GABAergic axon crossing by pairing two-photon glutamate uncaging with postsynaptic depolarization in CAI pyramidal cells. We found that repeated spine stimulation promoted growth of a GABAergic bouton onto the same dendrite. The dendritic feedback signal required postsynaptic activation of DAGL, which produces the endocannabinoid 2-AG, and was mediated by $\mathrm{CBI}$ receptors. Together, our findings reveal a dendritic signaling mechanism to trigger growth of an inhibitory bouton at dendritic locations with strong excitatory synaptic activity, which may serve to ensure inhibitory control over clustered excitatory inputs.

\section{Graphical abstract}

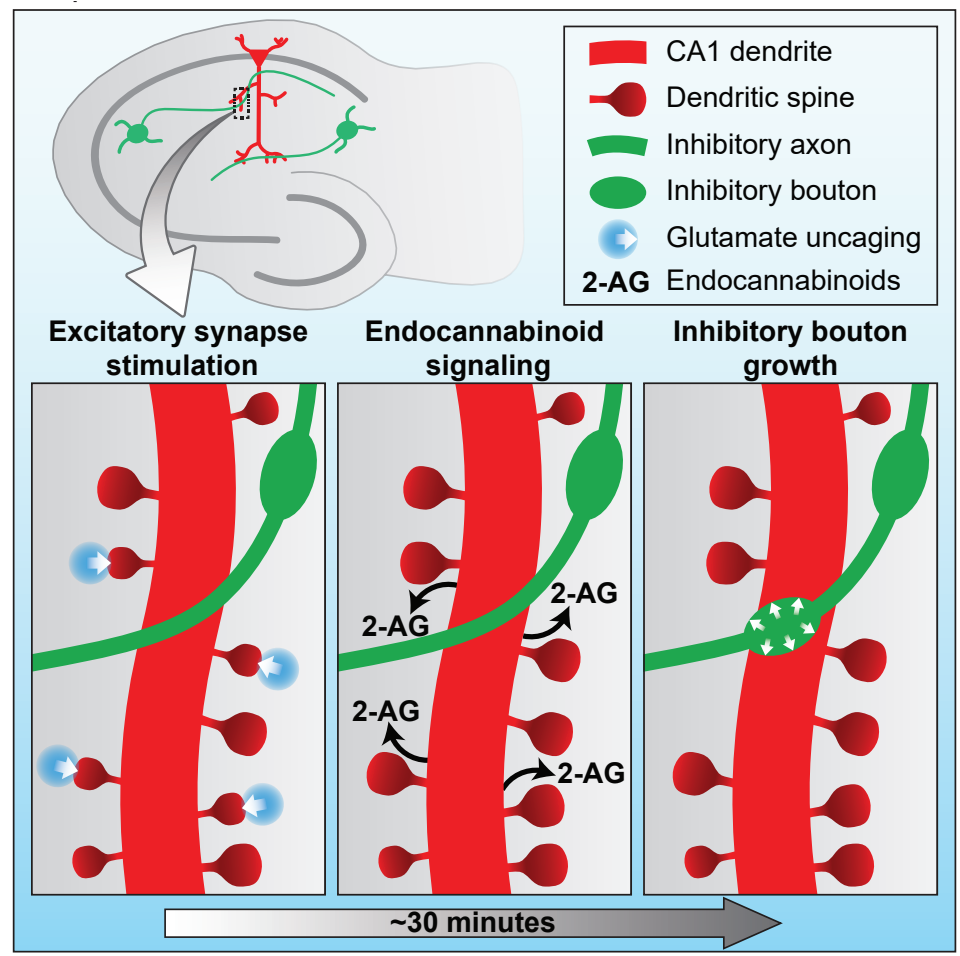




\section{Introduction}

Inhibitory synapses are crucial in shaping neuronal activity in the brain. The majority of inhibitory synapses are made onto postsynaptic dendrites (Megías et al., 200I), where they regulate the integration of incoming synaptic signals. Dendritic inhibitory synapses are an important component of nonlinear dendritic computation (Bloss et al., 2016; Schulz et al., 2018; Wilson et al., 20l8), and thereby essential for mediating complex behavior in vivo (Lovett-Barron et al., 20I4). As inhibitory synapses exert local control over calcium signals and ion channel opening with high temporal and spatial precision (Jadi et al., 2012; LovettBarron et al., 20I2; Müllner et al., 20I5), the precise location of inhibitory synapses within the dendrite is an important factor in determining their functional impact.

Dendritic inhibitory synapses are formed by the emergence of new GABAergic boutons at axonal locations with pre-established contact to the dendrite (Wierenga et al., 2008), but it remains unclear how the dendritic location of new inhibitory synapses is determined. In vivo studies have demonstrated that sensory activity can induce changes in dendritic inhibitory synapses (Froemke, 20I5; Keck et al., 20II), which are often coordinated with excitatory synapses on the same dendrite (Chen et al., 20I2, 20I5). It has been shown that excitatory synaptic activity affects the plasticity of existing dendritic inhibitory synapses (Bourne and Harris, 20I I; Chiu et al., 20I8; Petrini et al., 20I4), but it is not known if the formation of new dendritic inhibitory synapses can be coordinated by the dendrite.

Here we hypothesized that excitatory and inhibitory synapses are coordinated within dendrites to maintain a local balance of synaptic inputs. We used two-photon glutamate uncaging to stimulate individual spines on dendrites of CAI pyramidal neurons and observed that strong local excitation could trigger morphological changes in a GABAergic axon crossing the same dendrite. We found that the likelihood of GABAergic bouton growth was increased via local endocannabinoid signaling from the stimulated dendrite. Such a local retrograde signaling system may coordinate the number of excitatory and inhibitory synapses within a dendritic branch in an activity-dependent manner. 
A

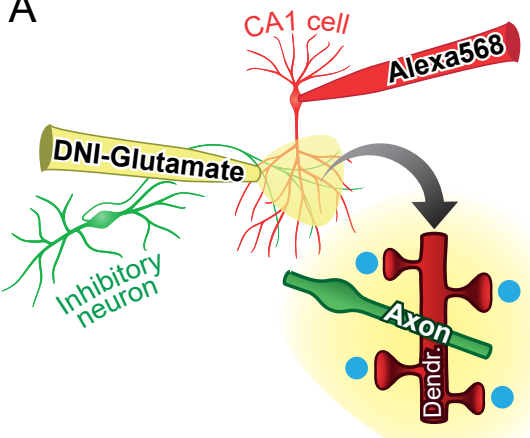

B

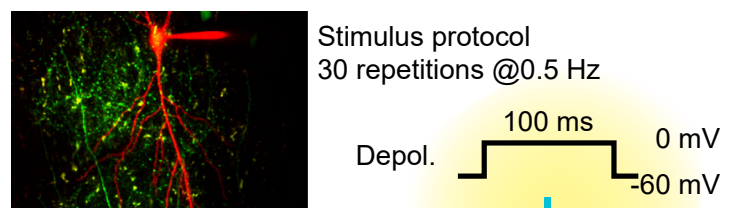

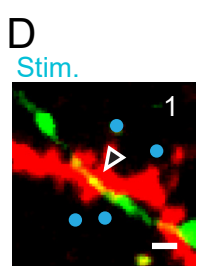

G

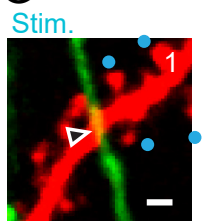

$\mathrm{J}$

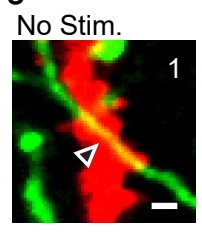

$\mathrm{M}$

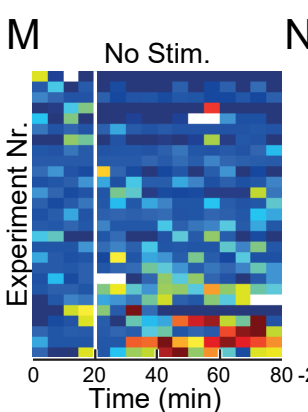

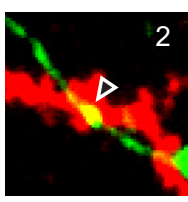

$\mathrm{E}$ (ㅇ) $t=5$ min $-1,-\infty$ 5 min 200 1,111111010011

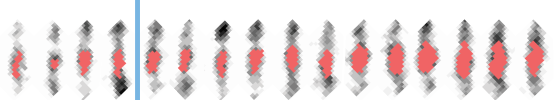
वे

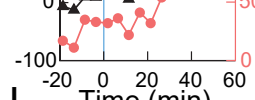<smiles>[CH]</smiles><smiles>C1C[C@H]2C[C@H]1C2</smiles>

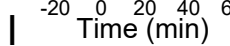
$4 \times 1 \mathrm{~ms}$
+ DNI-Glutamate
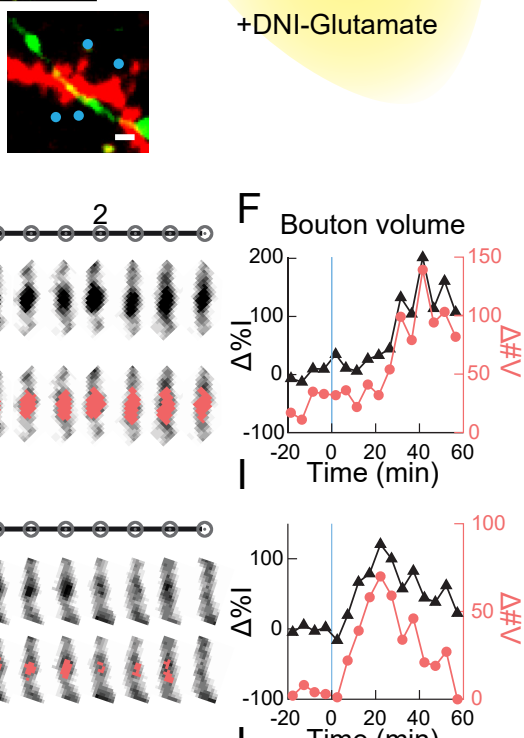
$L^{-20} \operatorname{Time}^{20}(\mathrm{~min})$

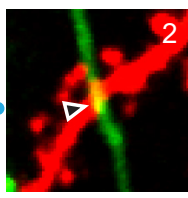
$\Delta t=5$ min ilinllitthith IIIIIIIIt)ill

$\mathrm{K}($ (2)

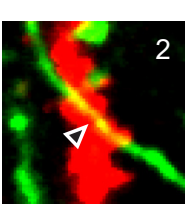

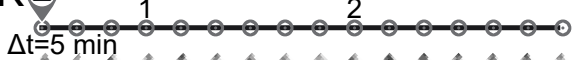
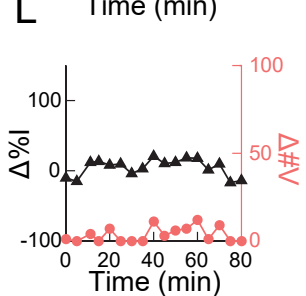

$\mathrm{N}$
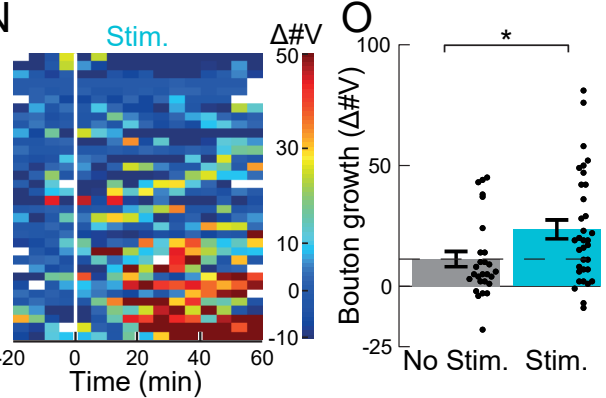

$\mathrm{P}$

$\stackrel{20 \quad 40 \quad 60}{\text { Time }}$ (min)

Figure I. Local dendritic stimulation promotes inhibitory bouton growth.

A: Schematic representation of the experimental setup. A CAI pyramidal cell was filled with Alexa568 via a patch pipette. DNI-glutamate was locally applied and glutamate uncaging was performed at four dendritic spines (blue dots) close to a crossing with a GFP-labeled inhibitory axon.

B: Overview image of an example experiment. In red, a pyramidal cell is visible with a patch pipette attached. The inset shows a dendrite with a crossing inhibitory axon (green). Blue dots indicate uncaging locations. Scale bar in inset is I $\mu \mathrm{m}$. 
C: The spine stimulation protocol consisted of repeated $(30 \mathrm{x} @ 0.5 \mathrm{~Hz})$ glutamate uncaging with postsynaptic depolarization to $0 \mathrm{mV}$. Uncaging was performed at four different spines near-simultaneously ( $\mathrm{ms}$ each; $\Delta \mathrm{t}=0$. I $\mathrm{ms})$.

D-F: Example of inhibitory bouton growth in response to local spine stimulation. D: Images of time points indicated in E.Arrowheads point towards the axon crossing, blue dots indicate uncaging locations. Scale bar is I $\mu \mathrm{m}$. E: Axonal segment at the crossing is displayed for all time points. The voxels above axon threshold are indicated in pink in the lower panel. F: Quantification of bouton volume over time, measured as the increase in number of voxels above axon threshold $(\triangle \# \mathrm{~V}$, pink) and increase in relative intensity $(\Delta \%$ l, black).

G-I: As in D-F, another example of inhibitory bouton growth after local spine stimulation.

J-L: As in D-F, no change in the axon in the absence of stimulation.

$\mathbf{M}, \mathbf{N}$ : Heat maps showing bouton volumes over time of all experiments with $(N ; N=34)$ and without $(M ; N=27)$ spine stimulation. Each row represents a single experiment. The vertical white line indicates the end of the baseline period (first 20 minutes). White squares indicate missing time points. Experiments are sorted by maximal bouton growth.

O: Bouton growth per experiment (averaged over four consecutive time points) for experiments with ('Stim') and without spine stimulation ('No Stim'). Dashed line indicates bouton growth in the absence of spine stimulation. Bars indicate mean \pm SEM; dots indicate independent experiments. *, $p<0.05$, Student's t-test.

P: Bouton growth occurrence over time in experiments in stimulated (Stim) and non-stimulated (No Stim) conditions. Bouton growth in the stimulated condition was different compared to baseline, as tested by Cochran's Q test followed by McNemar's test (time points with $p<0.05$ are indicated by blue line). For non-stimulated condition, bouton growth was not different from baseline $(p=0.10)$.

\section{Results}

To examine local coordination between dendritic excitatory and inhibitory synapses, we asked if inducing strong excitatory synaptic activity could trigger the formation of new inhibitory synapses on the same dendrite. We performed whole-cell patch clamp recordings of CAI pyramidal cells in organotypic hippocampal slices of GAD65-GFP mice (López-Bendito et al. 2004). In these mice, approximately $20 \%$ of the CAI interneurons are GFP-labeled, which target dendrites and mostly express reelin orVIP. Parvalbumin and somatostatin cells are not labeled (Wierenga et al., 20I0). The patch pipette contained the fluorescent red dye Alexa568 to visualize dendrites and spines of the recorded CAI pyramidal cell (Figure IA, B) (Müllner et al., 20 I5; Wierenga et al., 2008). We then searched the labeled dendrites of the recorded neuron for a crossing with a GFP-labeled axon without an inhibitory presynaptic bouton. After the acquisition of four baseline images ( $5 \mathrm{~min}$ intervals), four spines close to the green axon crossing (range I- $12 \mu \mathrm{m}$; mean $\pm \mathrm{SEM}=2.7 \pm 0.2 \mu \mathrm{m}$ ) were individually stimulated using two-photon glutamate uncaging, while the postsynaptic cell was depolarized to $0 \mathrm{mV}$ (Figure IC; see methods for details). After spine stimulation, we continued to monitor the axondendrite crossing for I hour ( 5 min intervals). We often observed a new inhibitory bouton forming at the crossing (Figure ID-I). In some cases, inhibitory boutons were formed de novo (Figure IG-I), while in other cases a small irregularity of the axon was already present during baseline, which grew into a bouton after spine stimulation (Figure ID-F). When we monitored inhibitory axon crossings at dendrites that were not stimulated, we only rarely observed spontaneous inhibitory bouton growth during the imaging period (Figure IJ-L), in agreement with our previous report (Wierenga et al., 2008). These observations suggest that the local activation of excitatory synapses can promote the growth of a nearby inhibitory bouton onto the same dendrite.

\section{Quantification of inhibitory bouton growth and time course}

We quantified the morphological changes in the inhibitory axon to directly compare inhibitory bouton growth in experiments with and without spine stimulation. We determined inhibitory bouton volume as the number of voxels above axon intensity (pink voxels in 
Figure IE, H, K, see methods for details; time course in Figure IF, I, L). We plotted the change in bouton volume for each time point in a heat map, in which each row represents an individual experiment (Figure IM, N), illustrating the substantial variability in size of individual boutons over time (Schuemann et al., 20I3; Wierenga et al., 2008). Inhibitory bouton growth was observed more often and more prolonged in experiments in which nearby spines were stimulated compared to experiments without stimulation. Maximal inhibitory bouton growth (averaged over 4 time points to avoid bias by single time point fluctuations) was significantly increased after spine stimulation compared to unstimulated controls (Figure IO). To summarize the time course of inhibitory bouton growth over all experiments, we plotted the fraction of experiments in which bouton growth was above threshold over time (Figure IP). In control experiments without stimulation, bouton growth occurrence did not deviate significantly from baseline during the entire imaging period. However, inhibitory bouton growth was significantly enhanced compared to baseline $>25$ minutes after spine stimulation. We verified that our conclusions did not depend on our quantification method (Figure SIA-D) or threshold (Figure SIE, F). Boutons formed within the short time frame of our experiments likely do not yet form mature inhibitory synapses (Schuemann et al., 2013;Wierenga et al., 2008). We have reanalyzed our previous data (Frias et al., 2019) and show that boutons can however acquire pre- and postsynaptic inhibitory proteins on this time scale (Figure SIG-I). Together, our data demonstrate that local stimulation of dendritic spines significantly increased the likelihood of growing a new bouton on an inhibitory axon crossing at the same dendrite.

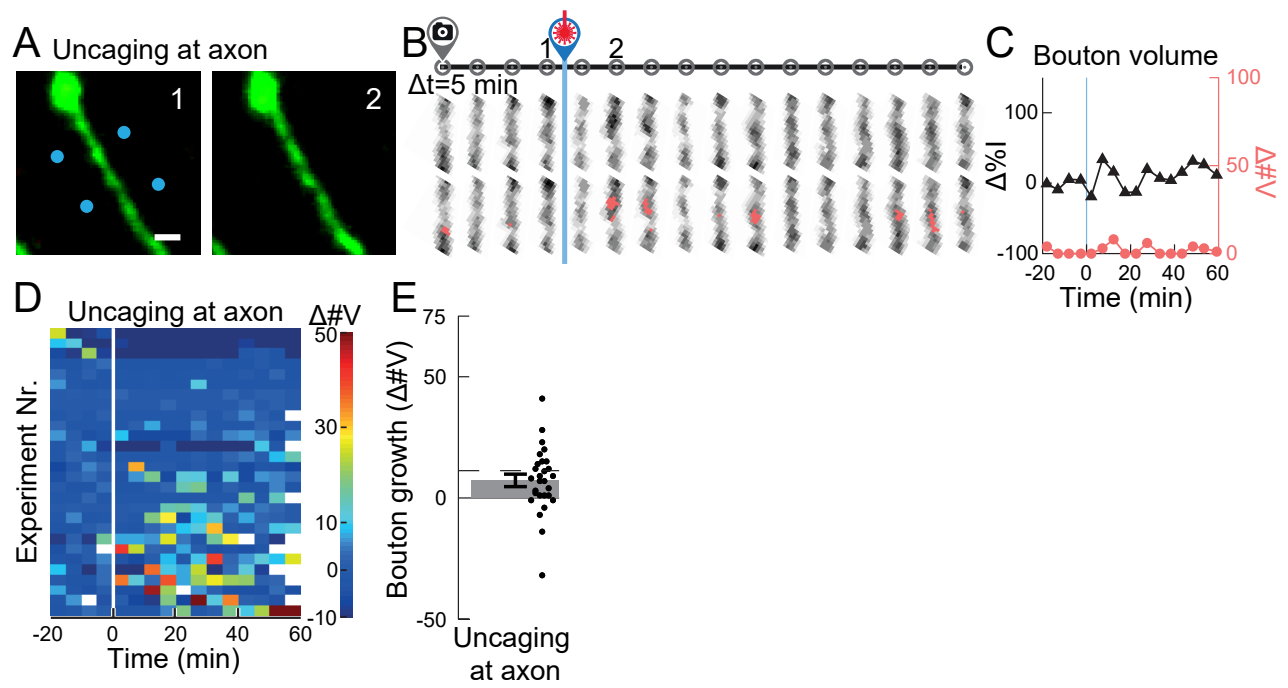

Figure 2. Uncaging glutamate directly at inhibitory axons does not induce bouton growth.

A-C: Example of experiment in which glutamate uncaging was performed near a GFP-labeled axon. A: Images of time points indicated in B. Blue dots indicate uncaging locations. Scale bar is I $\mu \mathrm{m}$. B: Axonal segment is displayed for all time points. The voxels above axon threshold are indicated in pink in the lower panel. C: Quantification of bouton volume over time, measured as the increase in number of voxels above axon threshold $(\Delta \# \mathrm{~V}$, pink) and increase in relative intensity $(\Delta \%$, black).

D: Heat map showing bouton volumes over time of all experiments $(\mathrm{N}=28)$. Each row represents a single experiment. The vertical white line indicates the end of the baseline period (first 20 minutes). White squares indicate missing time points. Experiments are sorted by maximal bouton growth.

E: Bouton growth per experiment (averaged over four consecutive time points) for glutamate uncaging at the axon. Dashed line indicates control bouton growth without spine stimulation (from Figure IO). Bars indicate mean \pm SEM; dots indicate independent experiments. $p=0.33$, Student's t-test, compared to control. 


\section{Inhibitory bouton growth is mediated by the postsynaptic dendrite}

We next asked if the postsynaptic dendrite has an active signaling role in triggering inhibitory bouton growth, or if the uncaged glutamate directly affects the inhibitory axon, for instance via presynaptic glutamate receptors. There was no correlation between inhibitory bouton growth and the distance from the uncaging locations to the axon (Figure S2), suggesting that glutamate diffusion to the axon is not a determining factor. We also directly tested whether inhibitory bouton growth could be induced by glutamate. We selected small segments of GFP-expressing inhibitory axons that were empty of boutons and performed two-photon glutamate uncaging at four locations close to the axon with the same stimulation protocol as used for spine stimulation (Figure 2A). While we observed fluctuations in axon intensity (Figure 2A-D), we never observed bouton growth beyond control levels (Figure 2D, E). These experiments demonstrate that local glutamate exposure to the axon itself is not sufficient to induce bouton growth, indicating that the signal required for inducing inhibitory bouton growth is generated by the stimulated dendrite.

\section{Inhibitory bouton growth is not tightly correlated with spine growth}

Local stimulation of individual spines by glutamate uncaging evoked synaptic currents, which were recorded at the soma (Figure 3A). When the four spines were stimulated together, we often observed a small supralinear summation (average $1.17 \pm 0.06$ ), reflecting the opening of dendritic voltage-gated ion channels or NMDA receptors (Harnett et al., 20I2; Losonczy and Magee, 2006; Weber et al., 2016).We did not find any correlation between this nonlinear component and inhibitory bouton growth (Figure 3B), suggesting that supralinear integration does not predict inhibitory bouton growth.

Our stimulation protocol was designed to induce strong local excitation within the dendrite. Uncaging was performed in normal ACSF (without TTX) and paired with postsynaptic depolarization to allow NMDA receptor activation. In accordance with previous reports using similar stimulation protocols (Govindarajan et al., 20I I; Tanaka et al., 2008), stimulated spines gradually increased in size during the first 10 minutes after the stimulation and then reached a plateau (Figure $3 \mathrm{C}$ ). When averaged over all stimulated spines, maximal spine size increased to $|3| \pm 2 \%$, compared to $\mid I 7 \pm 3 \%$ ( $p<0.00 \mid$, Student's t-test) for spines on non-stimulated dendrites. In general agreement with previous reports (Harvey and Svoboda, 2007; Oh et al., 20I5), $49 \%$ of spines grew after spine stimulation, compared to $25 \%$ of spines growing spontaneously in the absence of stimulation $\left(p<0.005\right.$, Pearson's $\chi^{2}$ test). To our surprise, we found no correlation between inhibitory bouton growth and the average growth of the four stimulated spines (Figure 3D), maximal spine growth (Figure S3A), or the number of growing spines (Figure S3B). This indicates that spine growth after local glutamate stimulation is not directly linked to nearby inhibitory bouton growth and suggests that local spine stimulation activates multiple signaling pathways in parallel.

\section{Inhibitory bouton growth requires NMDA receptor activation}

As we did not find a correlation between spine growth and bouton growth, we wondered whether inhibitory bouton growth may not require spine stimulation at all. Bouton growth may have directly resulted from the brief bouts of postsynaptic depolarization that were given during the spine stimulation protocol. We therefore imaged an axon-dendrite crossing as described before, but now applied the uncaging laser and postsynaptic depolarization in absence of DNI-glutamate ('Mock' stimulation). Repeated depolarizations did not induce 


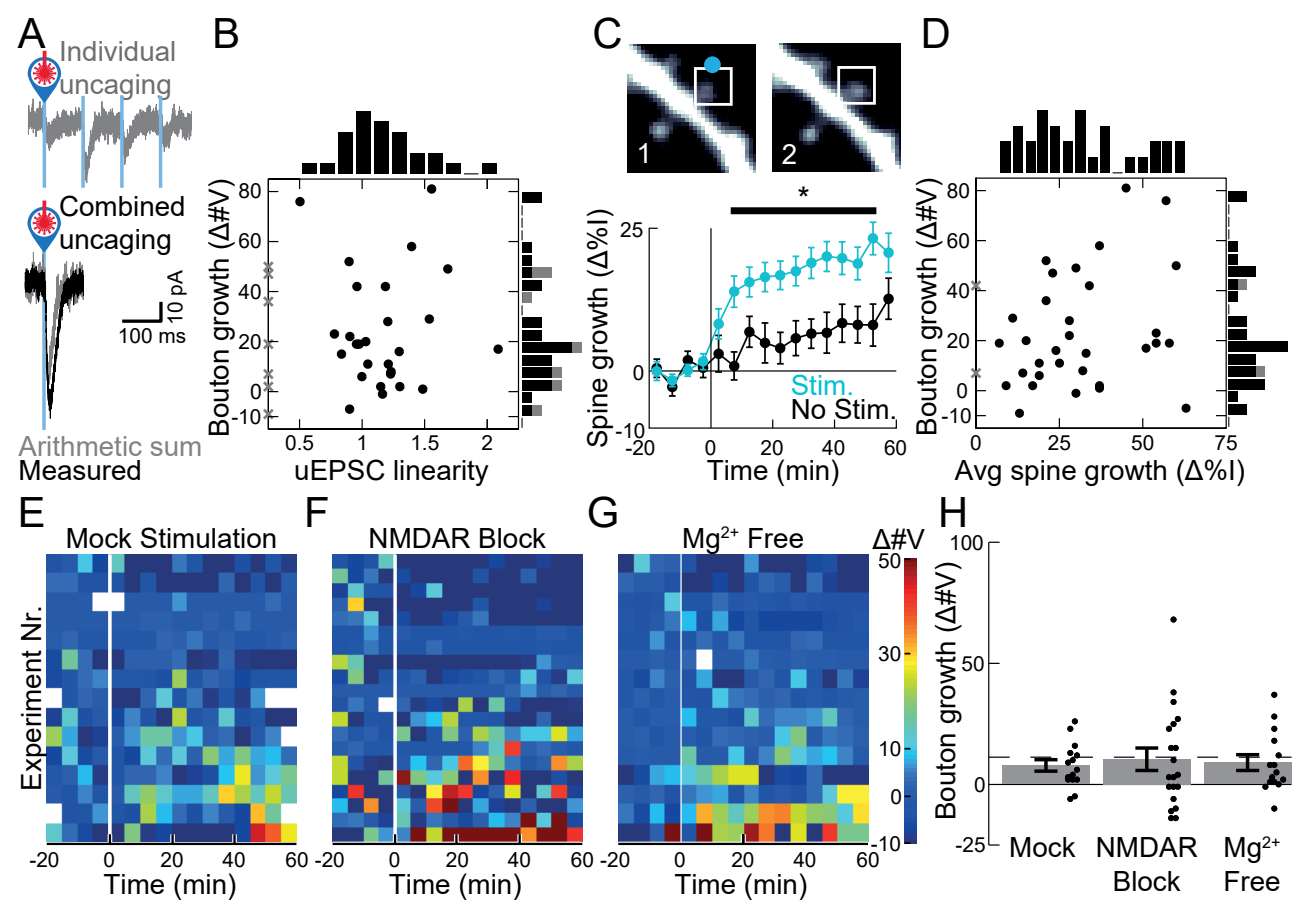

Figure 3. Inhibitory bouton growth requires NMDA receptor activation.

A: Top: postsynaptic currents induced by glutamate uncaging (uEPSC) at four spines stimulated sequentially. Bottom: arithmetic sum of the four spine responses (gray) and the measured uEPSC when stimulated near-simultaneously (black).

B: Scatter plot of uEPSC linearity against bouton growth for all experiments. Gray crosses indicate experiments where uEPSC linearity could not be quantified. Inhibitory bouton growth was not correlated with uEPSC linearity $(p=0.92$, Spearman). Distributions are shown as histograms next to the scatter plot.

C: Top: example of stimulated spine before (left) and after (right) the stimulus. The blue dot indicates the uncaging location. Bottom: spine growth, quantified as the increase in relative intensity $(\Delta \% l)$ over time. Local spine stimulation induced a gradual increase in spine size over time (blue; $n=6 I$ spines; $N=32$ ), which was absent in the non-stimulated condition (black; $n=104$ spines; $N=27)$. *, $p<0.05$ (Student's t-test with Bonferroni's correction). Error bars represent SEM.

D: Scatter plot of average spine growth against bouton growth for all experiments. Inhibitory bouton growth was not correlated with spine growth after local stimulation ( $p=0.22$, Spearman). Gray crosses indicate two experiments in which we could not determine spine volume. Distributions are shown as histograms next to the scatter plot.

E: Heat map showing bouton volumes over time of all experiments in which we performed the uncaging and depolarization stimulus in the absence of $\mathrm{DNl}$-glutamate ('Mock'; $\mathrm{N}=15$ ). Each row represents a single experiment. The vertical white line indicates the end of the baseline period (first 20 minutes). White squares indicate missing time points. Experiments are sorted by maximal bouton growth.

F: As in $E$, for experiments in the presence of $50 \mu M A P V(N=20)$.

G: As in $\mathrm{E}$, for experiments in ACSF without $\mathrm{Mg}^{2+}\left(\mathrm{Mg}^{2+}\right.$ free) and in the presence of $0.5 \mu \mathrm{MTTX}(\mathrm{N}=14)$.

H: Bouton growth (averaged over four consecutive time points) for the experiments shown in E-G. Dashed line indicates control bouton growth without spine stimulation (from Figure IO). Bars indicate mean \pm SEM; dots indicate independent experiments. p-values were $0.46,0.87$, and 0.65 (Student's t-test) for Mock, NMDAR Block and $\mathrm{Mg}^{2+}$ free experiments, compared to control.

spine growth (Figure S3C) and in the absence of glutamate receptor activation on spines, inhibitory bouton growth did not occur beyond control levels (Figure 3E, H and Figure S3D-F). This indicates that repeated postsynaptic depolarization by itself is not sufficient to induce inhibitory bouton growth. To specifically test for involvement of NMDA receptors, we repeated the glutamate uncaging experiments at four spines near an inhibitory axon crossing 
in the presence of $50 \mu \mathrm{M} A P V$ to prevent NMDA receptor activation. APV completely blocked the increase in spine size (Figure S3C) and inhibitory bouton growth (Figure 3F, H and Figure S3G-I), indicating that NMDA receptor activation was required.

To test if NMDA receptor activation was directly mediating inhibitory bouton growth, we repeated the uncaging experiments in $\mathrm{Mg}^{2+}$-free ACSF in the presence of TTX, allowing strong NMDA receptor activation during glutamate uncaging in the absence of postsynaptic depolarization. In accordance with previous reports (Harvey and Svoboda, 2007; Tanaka et al., 2008), spines which were stimulated in low $\mathrm{Mg}^{2+} / \mathrm{TTX}$ showed a rapid, immediate growth within 5 minutes after stimulation. Our spine stimulation protocol (30x I ms pulses @0.5 $\mathrm{Hz}$, without postsynaptic depolarization) induced clear spine growth in a similar number of spines compared to normal ACSF (55\% of stimulated spines were growing), but spine growth was mostly transient (Figure S3C). Interestingly, inhibitory bouton growth was not induced under these conditions (Figure 3G, H and Figure S3J-L). Altogether, these results show that local activation of glutamate receptors is required, but that receptor activation alone is not sufficient to trigger inhibitory bouton growth after local spine stimulation.
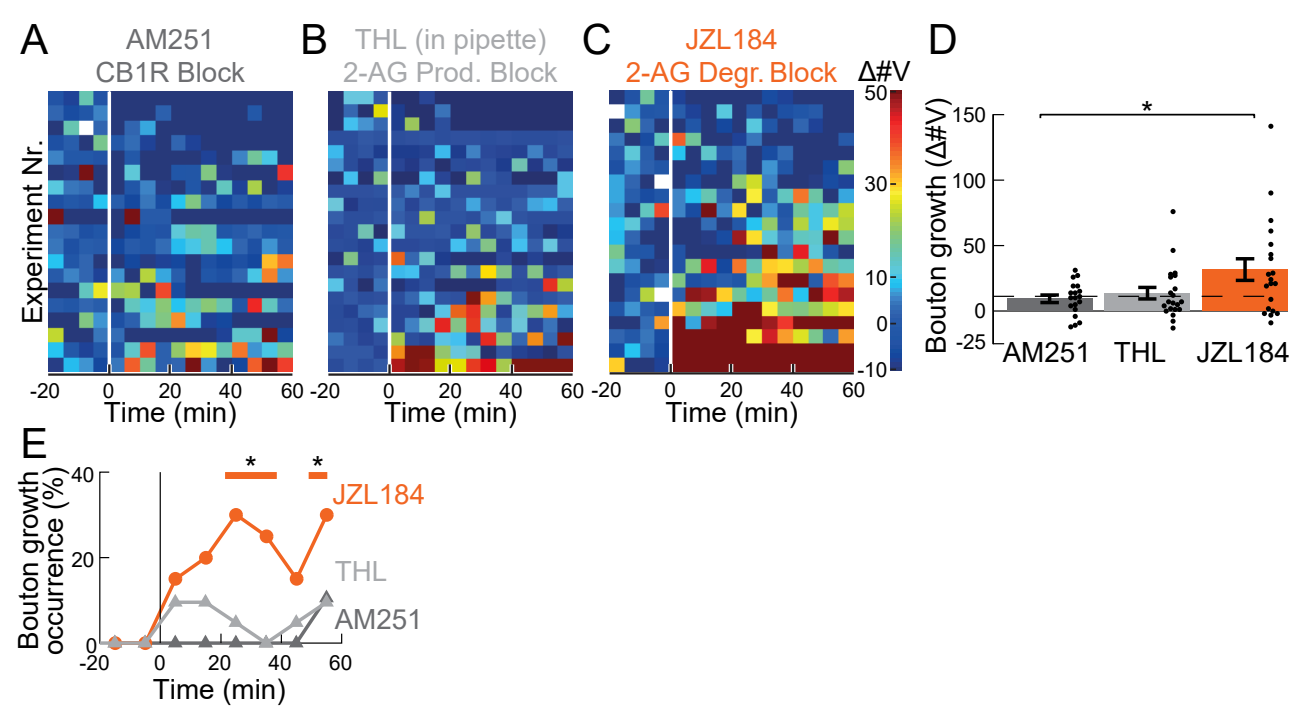

Figure 4: Inhibitory bouton growth is mediated by endocannabinoid signaling.

A: Heat map showing bouton volumes over time of all experiments in which spine stimulation was performed in the presence of $5 \mu \mathrm{M}$ of the $\mathrm{CBI}$ receptor antagonist $A M 25 \mathrm{I}(\mathrm{N}=19)$. Each row represents a single experiment. The vertical white line indicates the end of the baseline period (first 20 minutes). White squares indicate missing time points. Experiments are sorted by maximal bouton growth.

B: As in A, for experiments in which $5 \mu \mathrm{MTHL}$ was included in the patch pipette $(\mathrm{N}=2 \mathrm{I})$. THL is a lipase inhibitor with high affinity for the 2-AG production enzyme DAGL.

C: As in B, for experiments in the presence of $100 \mathrm{nM}$ JZLI84, an antagonist of the 2-AG degradation enzyme MAGL (N=20).

D: Bouton growth per experiment (averaged over four consecutive time points) for the experiments shown in A (dark gray, 'AM25I'), B (light gray, 'THL') and C (orange, 'JZLI84'). Dashed line indicates control bouton growth without spine stimulation (from Figure 10 ). Bars indicate mean $\pm S E M$; dots indicate independent experiments. Asterisk indicates $p=0.02$ for JZLI 84 compared to AM25 I ( $p=0.0$ I compared to control); $p=0.44$ THL vs AM 25 I; $\mathrm{p}=0.06 \mathrm{JZLI} 84 \mathrm{vs}$ THL (Student's $\mathrm{t}$ tests).

E: Bouton growth occurrence over time in experiments with AM25I (dark gray), THL (light gray) and JZLI84 (orange). Orange line indicates time points where bouton growth in JZLI84 was different compared to baseline, as tested by Cochran's $Q$ test $(p<0.0005)$ followed by McNemar's test $(p<0.05$ for individual time points). For AM25 I and THL, $\mathrm{p}=0.125$ and $\mathrm{p}=0.48$, respectively (Cochran's $\mathrm{Q}$ test). 


\section{Inhibitory bouton growth is mediated by retrograde endocannabinoid signaling}

Endocannabinoids, a well-known class of retrograde messengers, are active biolipids that are synthesized and released from the dendrite on demand (Castillo et al., 20 I2; Piomelli, 20 I4), and mediate synaptic changes via presynaptic CBI receptors (Cui et al., 20I6; Monday and Castillo, 2017). In our slices, $49 \pm 6 \%$ of GFP-labeled axons showed clear immunostaining for $\mathrm{CBI}$ receptors (Figure S4A-C). In accordance with in vivo data (Dudok et al., 20I5), CBI receptors uniformly decorated the entire surface of the axons, suggesting that $C B I$ signaling can occur in axonal stretches without boutons. Interestingly, we found that local spine stimulation could no longer trigger inhibitory bouton growth in the presence of AM25I, a $\mathrm{CBI}$ receptor antagonist (Figure 4A, D and Figure S4D-F), demonstrating the involvement of $\mathrm{CBI}$ receptors.

Hippocampal CAI dendrites and spines contain the enzyme DAGL, which produces the endocannabinoid 2-AG in an activity-dependent manner (Hashimotodani et al., 2007; Piomelli, 2014). We performed local spine stimulation experiments with THL, a lipase inhibitor with high specificity for DAGL, included in the patch pipette. Inhibiting postsynaptic DAGL blocked inhibitory bouton growth (Figure 4B, D and Figure S4G-I), suggesting that inhibitory bouton growth requires 2-AG release from the dendrite.

We also tested if we could facilitate inhibitory bouton growth by inhibiting the 2-AG degrading enzyme MAGL with JZLI84 (Cui et al., 2016). In the presence of JZLI84, local spine stimulation induced robust growth of nearby inhibitory boutons (Figure 4C, D and Figure S4J-L). Inhibitory bouton growth was comparable to experiments in the absence of pharmacological manipulation (Figure IO), although inhibitory bouton growth appeared to occur slightly earlier (Figure 4E). This experiment indicates that 2-AG degradation by MAGL does not limit the efficacy of the dendritic feedback signal. These results show that the dendritic feedback signal to induce inhibitory bouton growth after local spine stimulation requires $\mathrm{CBI}$ receptor activation and depends on postsynaptic 2-AG production. Together, this work demonstrates dendrites can trigger the growth of an inhibitory bouton at locations of strong excitatory synaptic activation through a local endocannabinoid-mediated feedback signal. 


\section{Discussion}

Dendritic inhibitory synapses are essential for dendritic computation as they can precisely shape local integration of excitatory synaptic inputs. Our data suggest that the formation of inhibitory synapses in the dendrite does not occur randomly, but that it can be directed by local excitatory synaptic activity in the dendrite. We describe a dendritic feedback signal to promote growth of inhibitory synapses in a dendritic region with strong synaptic activation. The signal from the dendrite to the axon requires local activation of glutamate receptors, including NMDA receptors. Furthermore, we show that inhibitory bouton growth after spine stimulation requires activation of $C B I$ receptors and depends on postsynaptic DAGL, the enzyme that produces the endocannabinoid 2-AG. The local signaling described here could provide inhibitory control at dendritic locations with strong excitatory synaptic activity and coordinate the number of excitatory and inhibitory synapses within dendrites in an activitydependent manner.

The formation of dendritic inhibitory synapses is a highly dynamic process, which takes several hours (up to a day) and bouton growth is generally the first step in this process (Dobie and Craig, 20I I; Villa et al., 2016; Wierenga et al., 2008). In the hours after initial bouton formation, presynaptic vesicles and postsynaptic gephyrin are slowly recruited (Frias et al., 2019), but not all newly formed boutons will stabilize and form functional inhibitory synapses (Schuemann et al., 2013; Wierenga et al., 2008). The molecular events that take place during this maturation process and the signaling pathways regulating it are only partially known (Flores et al., 2015; Frias et al., 2019; Krueger-Burg et al., 2017; Petrini et al., 20I4). The new boutons formed in our experiments will likely require several additional signals before becoming mature inhibitory synapses, which may depend on activity of nearby synapses or postsynaptic activity. Our current findings identify a triggering mechanism for the formation of new inhibitory boutons at active dendrites which requires $C B I$ receptor activation. $C B I$ receptors are mostly known for mediating synaptic weakening (Monday and Castillo, 2017). The mechanism described here could be related to the atypical endocannabinoid signaling that was recently found to mediate synaptic strengthening (Cui et al., 2016; Wang et al., 2017).

Our data suggest that the formation of inhibitory synapses is locally coordinated by the dendrite through postsynaptic endocannabinoid production. We showed that blocking 2-AG production by inhibiting postsynaptic DAGL interferes with inhibitory bouton growth. This suggests that 2-AG is the main endocannabinoid involved, but we do not rule out an additional role for other endocannabinoids (Piomelli, 20I4). During clustered spine stimulation, the postsynaptic production of endocannabinoids is likely triggered by synergistic action of voltage-dependent mechanisms and activation of local glutamate receptors (possibly both synaptic and extrasynaptic), as we found that each of these factors alone was not enough to trigger the dendritic feedback signal (Figure 3). Coincidence of multiple postsynaptic signals may be required to boost DAGL activity (Cui et al., 2016; Jung et al., 2012; Younts et al., 2013). Postsynaptic depolarization may be provided in vivo by action potential firing (Stuart et al., 1997) or plateau potentials (Gambino et al., 2014). Please note that in the low $\mathrm{Mg}^{2+}$ / TTX condition, cell adhesion, neurotransmitter release or presynaptic activity may also have changed. The precise signaling pathways and the optimal conditions to induce the dendritic feedback signal should be addressed in future experiments.

Dendrites receive and integrate synaptic signals from many different presynaptic partners. 
Inhibitory synaptic plasticity can depend on inhibitory neuron subtype (Chiu et al., 2018). Although we did not explicitly address this here, it is possible that regulation of inhibitory bouton growth via $\mathrm{CBI}$ receptors is specific for a subset of inhibitory axons. It needs to be determined if perisomatic inhibitory synapses, which can also express high levels of CBI receptors (Hartzell et al., 2018), can be regulated by a similar mechanism.

Our study suggests dendrites can actively organize incoming axons by sending a retrograde signal to promote the formation of specific inhibitory synapses at locations where clusters of excitatory synaptic inputs are strongly activated. Local clustered activation of inputs likely happens during physiological activation, as synaptic inputs with similar properties or activity patterns are often clustered on the same dendritic branch in vivo (Bloss et al., 2016; lacaruso et al., 2017; Wilson et al., 2016). Clustering of excitatory inputs enhances the computational capacity of postsynaptic neurons (Branco and Häusser, 20 I0; Poirazi and Mel, $200 \mathrm{I})$ and inhibitory synapses at excitatory clusters will provide important local control over computations performed by individual dendritic branches (Bloss et al., 2016; LovettBarron et al., 20I2; Müllner et al., 20I5). The dendritic signaling mechanism described here would enable fine-tuning of dendritic inhibitory synapses in response to changes in activity of synaptic input clusters, allowing adaptation of dendritic inhibition during learning. 


\section{Methods}

\section{Experimental model and subject details}

\section{Mice}

In this study, male and female GAD65-GFP mice (López-Bendito et al., 2004) were used (bred as a heterozygous line in a C57BL/6JRj background). GAD65-GFP mice express GFP in $\sim 20 \%$ of GABAergic interneurons in the CAI region of the hippocampus. The majority of GFP-labeled interneurons target dendrites and express reelin and VIP, while parvalbumin and somatostatin expression is nearly absent (Wierenga et al., 20I0). We typically do not see many GFP-labeled boutons around the somata of CAI pyramidal cells, indicating that basket cells are mostly not labeled in our slices. The sparse GFP expression allows monitoring of morphological changes in individual inhibitory axons (Schuemann et al., 2013;Wierenga et al., 2008). All animal experiments were performed in compliance with the guidelines for the welfare of experimental animals issued by the Federal Government of The Netherlands. All animal experiments were approved by the Animal Ethical Review Committee (DEC) of Utrecht University.

\section{Slice cultures}

Organotypic hippocampal slice cultures were prepared at postnatal day 6-8 with a method slightly modified from Stoppini et al. (Stoppini et al., 199I). Mice were decapitated, followed by removal of the brain. The brain was placed in ice cold Gey's Balanced Salt Solution (GBSS, consisting of $(\mathrm{mM})$ : $137 \mathrm{NaCl}$. $5 \mathrm{KCl}$, I.5 $\mathrm{CaCl}_{2}, \mathrm{I} \mathrm{MgCl}_{2}, 0.3 \mathrm{MgSO}_{4}, 0.2 \mathrm{KH}_{2} \mathrm{PO}_{4}, 0.85$ $\mathrm{Na}_{2} \mathrm{HPO}_{4}$ ) supplemented with $12.5 \mathrm{mM}$ HEPES, $25 \mathrm{mM}$ glucose and I mM kynurenic acid ( $\mathrm{pH}$ set at 7.2, osmolarity set at $320 \mathrm{mOsm}$, sterile filtered). Under sterile conditions, the frontal part of the brain and the cerebellum were dissected along the transverse plane and removed.The two hemispheres were then separated along the midline. For each hemisphere, the midbrain was carefully removed and two incisions were made at the rostral and caudal ends of the hippocampus. The hippocampus was then carefully rolled out by flipping it 180 degrees over its long axis and a parallel incision was made in the cortex to dissect the hippocampus. Both hippocampi were placed in parallel on a PVC disk, excess liquid was removed, and slices were chopped perpendicularly to the long axis of the hippocampus with a thickness of $400 \mu \mathrm{m}$ using a Mcllwain Tissue Chopper. Slices were placed back in GBSS solution and carefully separated from each other. If needed, excess cortical tissue was removed from individual slices. Slices were washed in culturing medium (consisting of 48\% MEM, 25\% HBSS, 25\% horse serum, $30 \mathrm{mM}$ glucose and $12.5 \mathrm{mM} \mathrm{HEPES,} \mathrm{pH} \mathrm{set} \mathrm{at} \mathrm{7.3-}$ 7.4 and osmolarity set at $325 \mathrm{mOsm}$ ) before being placed on Millicell cell culture inserts (Millipore) in 6-well plates containing culturing medium. Slices were stored in an incubator $\left(35{ }^{\circ} \mathrm{C}, 5 \% \mathrm{CO}_{2}\right)$ until use and culturing medium was completely replaced twice a week. Over time, slices attach to the membrane, flatten, and continue to develop in a physiological manner (De Simoni et al., 2003). Slices used in experiments were kept at least 7 days in vitro (DIV; average slice age was I4.4 DIV (with standard deviation=3.9; range 7-2I)). There was no correlation between inhibitory bouton growth and slice age. 


\section{Method details}

\section{Two-photon microscopy and electrophysiology}

Before the start of each experiment, a hippocampal slice was transferred, together with the piece of membrane it was plated on, from the incubator to the microscope recording chamber. During the experiment, the slice was perfused with carbogenated $\left(95 \% \mathrm{O}_{2}, 5 \%\right.$ $\mathrm{CO}_{2}$ ) artificial cerebrospinal fluid (ACSF, consisting of (mM): $126 \mathrm{NaCl}, 3 \mathrm{KCl}, 2.5 \mathrm{CaCl}_{2}, 1.3$ $\mathrm{MgCl}_{2}, 26 \mathrm{NaHCO}_{3}, \mathrm{I} .25 \mathrm{Na}_{2} \mathrm{H}_{2} \mathrm{PO}_{4}, 20$ glucose and I Trolox) at a rate of approximately I $\mathrm{ml} / \mathrm{min}$ at room temperature. Two-photon imaging was performed on a customized twophoton laser scanning microscope (Femto2D, Femtonics Ltd., Budapest, Hungary) using a TiSapphire femtosecond pulsed laser (MaiTai, Spectra-Physics). This laser, tuned at 840 or $\mathbf{8 7 0}$ $\mathrm{nm}$, was used to excite GFP and Alexa568 simultaneously and fluorescence was detected using two GaAsP photomultiplier tubes.

A 4x air objective (Nikon Plan Apochromat) was used to locate the CAI region of the slice culture and to roughly position the pipettes for whole-cell patch clamp (thick-walled borosilicate glass, World Precision Instruments) and for local DNI-glutamate application (thin-walled borosilicate glass, World Precision Instruments) using micromanipulators (LN Junior, Luigs \& Neumann). Under a 60x water immersion objective (Nikon NIR Apochromat; $N A=I .0$ ), the opening of the application pipette was enlarged to approximately $5-10 \mu \mathrm{m}$ by carefully tapping it against the patch pipette, and both pipettes were placed close to the imaging area. Whole-cell patch clamp of a CAI pyramidal cell neuron was performed with the patch pipette (3-7 $\mathrm{M} \Omega$ ) filled with internal solution (consisting of $(\mathrm{mM})$ : I $40 \mathrm{~K}$-gluconate, $4 \mathrm{KCl}, 0.5$ EGTA, I0 HEPES, 4 MgATP, $0.4 \mathrm{NaGTP}, 4 \mathrm{Na}_{2}$ Phosphocreatine, and 30 uMAlexa568 (Thermo Fisher Scientific)). Cells were excluded when the initial resting membrane potential exceeded $-50 \mathrm{mV}$, if the cell was firing spontaneously, or if Rs exceeded $30 \mathrm{M} \Omega$. The pyramidal cell was kept in voltage clamp at $-60 \mathrm{mV}$ throughout the experiment.

Crossings between the Alexa568-labeled dendrite and a GFP-labeled axon were identified using two-photon microscopy by overlap between the red and green channel (Müllner et al., 20I5; Wierenga et al., 2008). After finding an inhibitory axon crossing with no bouton, the application pipette was placed with the opening towards the crossing (at $\sim 40 \mu \mathrm{m}$ distance and $\sim 20 \mu \mathrm{m}$ above the surface of the slice). The time needed for the search procedure and pipette placement was typically 10-15 minutes, and less than 30 minutes in all experiments. In most cases, dendrites were in stratum radiatum, with some experiments performed in stratum lacunosum moleculare. Image stacks of the axon-dendrite crossing were made every 5 minutes at a resolution of 9 pixels $/ \mu \mathrm{m}$ with $0.5 \mu \mathrm{m}$ z-steps $(256 \times 256$ pixels, $28.4 \times 28.4 \mu \mathrm{m})$.

\section{Spine stimulation}

After acquiring four baseline time points, DNI-glutamate-TFA (Femtonics Ltd. (Tønnesen et al., 20 I4), 5 mM dissolved in HEPES-ACSF: (mM) I $35 \mathrm{NaCl}, 3 \mathrm{KCl}, 2.5 \mathrm{CaCl}_{2}$, I.3 $\mathrm{MgCl}_{2}, \mathrm{I} .25$ $\mathrm{Na}_{2} \mathrm{H}_{2} \mathrm{PO}_{4}, 20$ Glucose, and 10 HEPES) was locally applied to the axon-dendrite crossing using a Picospritzer II (General Valve Corporation; 8- $12 \mathrm{mmHg}$ ) through the application pipette.A second Ti-Sapphire femtosecond pulsed laser (MaiTai, Spectra-Physics) tuned at $730 \mathrm{~nm}$ was used for two-photon uncaging. We selected four small spines close (range I- $12 \mu \mathrm{m}$; average $2.7 \pm 0.2 \mu \mathrm{m}$ ) to the axon crossing for stimulation. Usually spines were selected at both sides of the axon crossing. We optimized the excitation wavelength for visualizing changes in GFP-labeled axons, which may have compromised our ability to visualize the smallest 
spines. After locally applying caged glutamate for $\sim$ I minute, the laser scanned across lines of $\sim 0.5 \mu \mathrm{m}$ long and $\sim 0.5 \mu \mathrm{m}$ away from the spines for I ms. Laser intensity for glutamate uncaging was carefully chosen to evoke excitatory postsynaptic currents with physiological amplitude at individual spines (mean amplitude = $10.9 \pm 0.6 \mathrm{pA}$; range $4-18 \mathrm{pA}$ ), measured at the soma. The amplitude of uncaging currents were not affected much by the presence of NMDA receptor antagonist APV (mean amplitude $=9.2 \pm 0.8 \mathrm{pA} ; \mathrm{p}=0.1 \mathrm{I}$, Student's $\mathrm{t}$-test), in accordance with only a small NMDA receptor contribution to the synaptic current.

We also recorded the combined uEPSC when all four spines were stimulated together nearsimultaneously $(0.1 \mathrm{~ms}$ between spines). After recording the combined uEPSC, the spine stimulation protocol was performed.The four spines were stimulated together (I ms pulses with $0.1 \mathrm{~ms}$ interval between spines) while the postsynaptic cell was depolarized to $0 \mathrm{mV}$, and this stimulation was repeated 30 times at $0.5 \mathrm{~Hz}$ (Figure IC). Afterwards, the combined and individual uEPSCs were measured again. Experiments were only included if uEPSCs could be evoked after the stimulation protocol, verifying that glutamate uncaging had been successful. uEPSC linearity was determined by dividing the uEPSC amplitude of the combined response by the arithmetic sum of individual uEPSC amplitudes.

For glutamate uncaging close to GFP-labeled axons, we selected small segments of GFPexpressing inhibitory axons that were empty of boutons and performed two-photon glutamate uncaging at four locations close to the axon with the same stimulation protocol as used for spine stimulation (30x Ims pulses @0.5 Hz at four locations, interval between locations $0.1 \mathrm{~ms}$ ). Glutamate uncaging spots were located close to the axon, at an average distance of $1.2 \pm 0.2 \mu \mathrm{m}$. Even though there will be many unlabeled spines in close proximity to the uncaging spots, the likelihood that this uncaging protocol will activate multiple spines on a single dendrite will be low.

To block NMDAR activation, $50 \mu \mathrm{M}$ DL-APV (APV, Tocris) was added to regular ACSF and was applied to the slice during the entire experiment. To perform two-photon uncaging in the absence of postsynaptic depolarization, regular ACSF was replaced by $\mathrm{Mg}^{2+}$-free ACSF (regular ACSF without $\mathrm{MgCl}_{2}$ ) containing $0.5 \mu \mathrm{M}$ tetrodotoxin (TTX, Abcam). $\mathrm{Mg}^{2+}$-free ACSF was washed in before imaging started. Regular ACSF was washed back in after the uncaging stimulus at $\sim 15$ minutes. For these experiments, DNI-glutamate was dissolved in HEPES-ACSF without $\mathrm{MgCl}_{2}$ and containing $0.5 \mu \mathrm{MTTX}$.

To block CBI receptors, $5 \mu$ MAM25 I (Tocris) was added to the bath and allowed to incubate for at least 40 minutes before the start of the experiment (so at least 60 minutes before spine stimulation). We also added $10 \mu$ M AM25I in the application pipette. To block 2-AG production by the enzyme DAGL in the postsynaptic dendrite, $5 \mu \mathrm{M}$ of Tetrahydrolipstatin (THL, Orlistat, Sigma) was added to the patch clamp intracellular solution. The time between break-in and spine stimulation was typically 30-40 minutes. To block 2-AG degradation, 100 nM JZLI 84 (Tocris) was added to the bath and allowed to incubate for at least 40 minutes before the start of the experiment.

\section{Correlating boutons with synaptic markers}

For the data shown in Figure SII-K, image stacks of multiple GFP-labeled axons were acquired at $910 \mathrm{~nm}$ for 15 time points $(\Delta \mathrm{t}=10$ minutes) at a resolution of 12 pixels $/ \mu \mathrm{m}$ with $0.5 \mu \mathrm{m}$ z-steps (I I $24 \times$ I I 24 pixels, $93.5 \times 93.5 \mu \mathrm{m}$ ), as previously described (Frias et al., 2019).After the experiment, an autofluorescent laser "scar" was made by performing a line 
scan at high intensity to mark the imaged region. The slice was then fixed and processed for immunohistochemistry and confocal imaging.

\section{Immunohistochemistry and confocal microscopy}

Slices were fixed by placing them in a $4 \%$ paraformaldehyde solution for 30 minutes at room temperature. After washing them thoroughly in phosphate buffered saline (PBS), the slices were permeabilized for 15 minutes in $0.5 \%$ Triton-X 100 in PBS for 15 minutes, followed by $I$ hour in a blocking solution consisting of $10 \%$ normal goat serum and $0.2 \%$ Triton-X 100 in PBS. Primary antibody solution was applied at $4{ }^{\circ} \mathrm{C}$ overnight. Slices were washed thoroughly in PBS and placed in secondary antibody solution for four hours at room temperature. Finally, slices were washed thoroughly in PBS and mounted in Vectashield mounting medium (Vector labs). The following primary antibodies were used: mouse $\alpha-C B I R$ (Synaptic Systems 2580 I I; 1:500), rabbit $\alpha$-VGAT (Synaptic Systems 13। 003; I:1000) and mouse $\alpha$-gephyrin (Synaptic Systems I47 0I I; I:I000). Secondary antibodies were Alexa-405 and Alexa-594 conjugated goat antibodies (Invitrogen). Confocal images were taken on a Zeiss LSM-700 confocal laser scanning microscopy system with a Plan-Apochromat 63x I.4 NA oil immersion objective. Image stacks were acquired at I0-13 pixels per $\mu \mathrm{m}$ and with $0.3 \mu \mathrm{m} \mathrm{z}$ steps.

\section{Quantification and statistical analysis}

\section{Quantification of bouton and spine volumes}

Experiments in which we imaged axon-dendrite crossings were only included in the analysis when the local maxima of the red and the green channel were not more than one optical section $(\Delta z=0.5 \mu \mathrm{m})$ apart (Müllner et al., 2015; Wierenga et al., 2008). For simplicity, we refer to all axonal swellings or varicosities as boutons, although we are aware that a small fraction might not contain presynaptic specializations (Wierenga et al., 2008). Analysis and bouton volume quantification was performed on median filtered images using custom written Matlab scripts (Mathworks).A box of $9 \times 9 \times 5$ voxels (i.e. I $1 \times 2.5 \mu \mathrm{m}$ ) was positioned manually at the axon-dendrite crossing at each time point. An axon threshold was set to separate the boutons from the axon, relative to the axon intensity (determined from the summed projection of 5 z-planes, modus along the entire axon) to account for possible intensity fluctuations between time points and differences between experiments (axon threshold was $2.9 \pm 0.1$ for non-stimulated axons; $2.8 \pm 0$. I for stimulated axons; $p=0.19$, Student's $t$ test). In a few experiments, photobleaching correction was applied. The absolute change in bouton volume $(\triangle \# \mathrm{~V})$ was quantified as the number of voxels above bouton threshold minus the average volume of the baseline period. The relative change in bouton intensity $(\Delta \% \mathrm{l})$ was defined as the integrated intensity of all voxels inside the same $9 \times 9 \times 5$ box, divided by the average intensity of the baseline period. As baseline intensity of the axon was sometimes very low, $\Delta \%$ gave very high values for boutons that were growing from dim axons. To avoid strong bias for these events, we used the absolute measure $\triangle \# \mathrm{~V}$ for our comparisons. We verified that the two measures $\Delta \# \mathrm{~V}$ and $\Delta \% \mathrm{l}$ were highly correlated (Pearson's $r=0.82$, $\mathrm{p}<0.000 \mathrm{I}$ ) and that our conclusions do not critically depend on our quantification method (Figure SI). Bouton growth per experiment was defined as the maximum bouton volume after the baseline averaged over four consecutive time points. To show the average time course of bouton growth, we determined bouton growth occurrence as the fraction of experiments in which bouton growth exceeded an empirically chosen threshold ( 35 voxels for $\triangle \# \mathrm{~V}$ and $50 \%$ for $\Delta \% \mathrm{l})$. We verified that our conclusions do not depend on the choice of this threshold (Figure SIE, F). Bouton growth occurrence was plotted in bins of 10 minutes 
to reduce noise and reduce the weight of boutons which last for only one time point.

Spine growth was determined as relative change in intensity $(\Delta \%)$ over a volume of $9 \times 9 \times 5$ voxels and spines were considered to grow when $\Delta \% \mathrm{I}>25 \%$. Some stimulated spines which overlapped with the shaft or with neighboring spines were not analyzed. Maximal and average spine growth was determined as the maximum and mean of the four stimulated spines per experiment. Baseline spine volumes were similar between conditions $(p=0.7 \mathrm{I}$, Student's t-test). Spine and bouton growth were induced in whole-cell recordings even after 20-40 minutes. We found no correlation between maximal spine growth or bouton growth with the time after break-in (Figure SIJ, K).

\section{VGAT and gephyrin analysis}

For Figure SII-K, we reanalyzed data from our previous work (Figure I in Frias et al., 2019). Individual boutons in GFP-labeled axons were scored for presence or absence at all 15 time points of the live imaging using custom-made Matlab software. Using the laser scar and GFP fluorescence as a guide, the same boutons were identified in the fixed tissue and presence of presynaptic vesicular GABA transporter VGAT or postsynaptic scaffold protein gephyrin was determined using confocal microscopy. For the current analysis, we selected boutons which matched the time course of local spine stimulation experiments. Selected boutons were present for at least 2 time points in the last hour of imaging (TPI0 to TPI5; 20-60 minutes in total). Boutons which were already present for more than 3 time points during the first 9 time points were excluded. We determined the fraction of selected boutons that were positive for VGAT or gephyrin.

\section{Quantification of CBIR-positive axon fraction}

In Fiji/lmage, all healthy looking axons in a maximum intensity projection (5-10 images) were manually selected based on the GFP channel. For each axon, colocalization with the $\mathrm{CBI}$ receptor channel was determined. Per image stack, two or three maximum intensity projections were analyzed. The total amount of CBI receptor-positive GFP axons in an image stack was divided by the total amount of quantified GFP-positive axons to determine the $\mathrm{CBI}$ receptor-positive fraction.

\section{Statistical analysis}

Statistical differences between groups were tested with two-sample Student's t test and means are given \pm standard error of the mean (SEM), unless otherwise stated. Bouton growth occurrence per condition was tested for statistical significance against its own baseline period with a Cochran's $Q$ test, followed by a post-hoc McNemar's test. Fractions of growing spines between conditions were tested with Pearson's $\chi^{2}$ test. Possible correlations were tested with Spearman's ranked test and Pearson's correlation coefficient $(r)$ was determined. Statistical analyses were performed using Matlab R2013a, Graphpad Prism 7, and SPSS Statistics 24. 


\section{Acknowledgments}

The authors thank René van Dorland for excellent technical support. This research was supported by the Netherlands Organization for Scientific Research, as part of the VIDI program (CJW,HYH; \#0 I6.I 26.36 I), and as part of the research programme of the Foundation for Fundamental Research on Matter (FOM) (DLHK; \#I5PR3I78-I), by the programme 34 FP7/2007-20I3/ under REA grant agreement 28958I (CPF) and by ERCVisionby3DSTIM, KFII6-I-20I6-0I77, NVKPI6-I-20I6-0043, GINOP2.I.I-I5-20I6-00979 (BR).

\section{Author contributions}

CJW conceived and supervised the study. CJW, HYH and DLHK designed the experiments. $\mathrm{HYH}$ and DLHK performed the experiments and analysis. CPF performed and analyzed the experiments presented in Figure SIG-I. BR developed and provided DNI-glutamate, $\mathrm{CCH}$ provided critical input. CJW, HYH and DLHK wrote the paper with critical input from all authors.

\section{Declaration of interests}

$\mathrm{BR}$ is one of the founders of Femtonics $\mathrm{Kft}$. and is a member of its scientific advisory board. 
Endocannabinoid signaling mediates local dendritic coordination between excitatory and inhibitory synapses 


\section{Supplementary Figures}
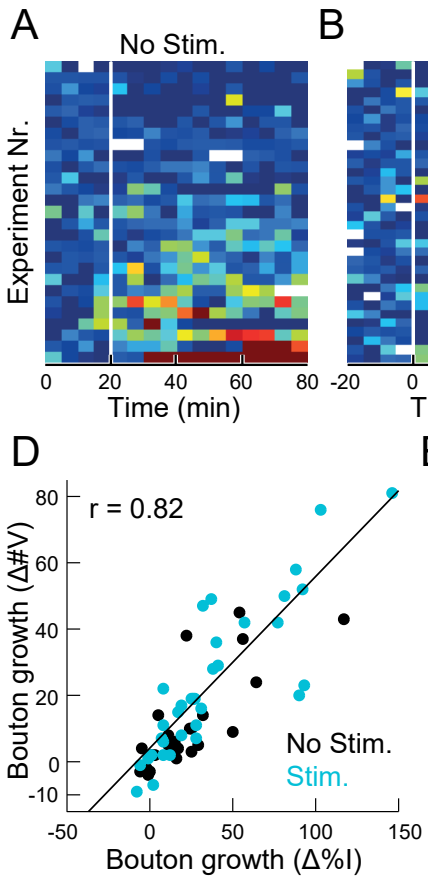

B
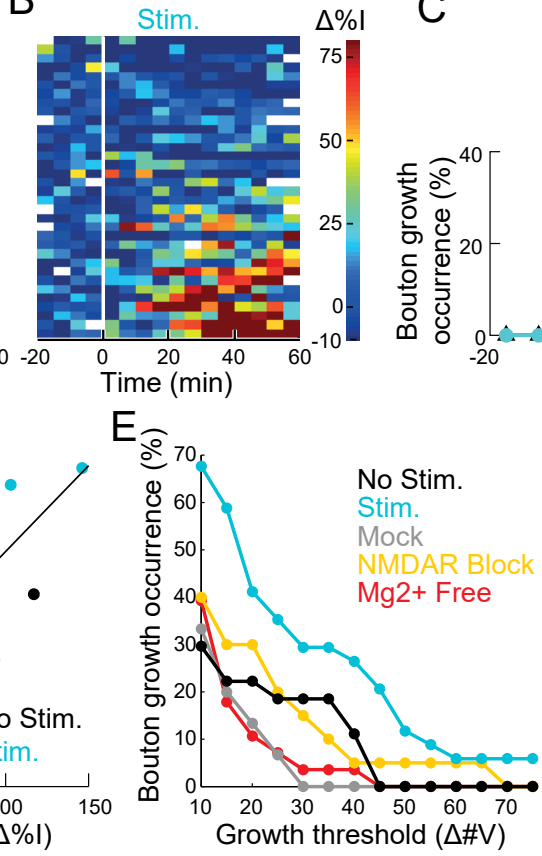

C
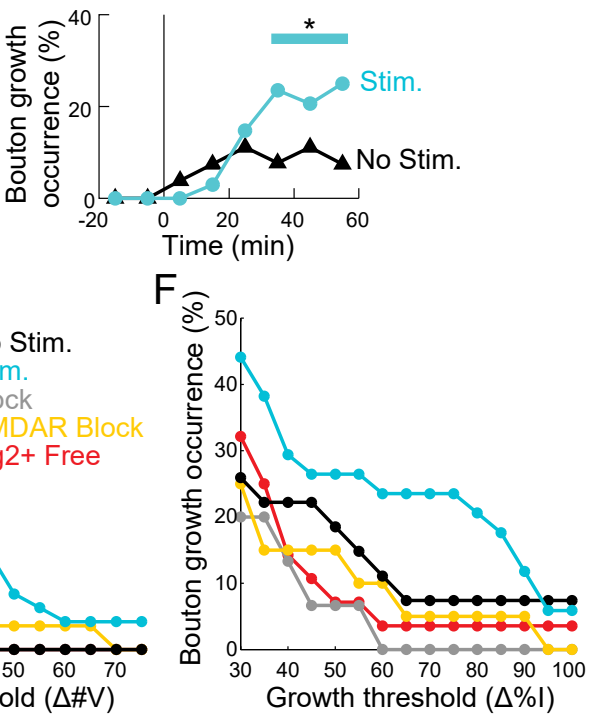

G 2P (pre) 2P (post) Confocal (GFP/NGAT)

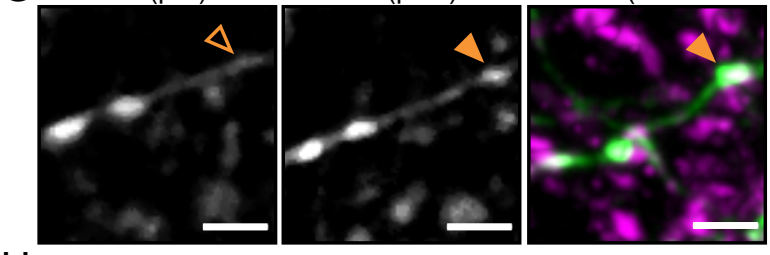

$\mathrm{H}$

$2 \mathrm{P}$ (post) Confocal (GFP/Geph.)
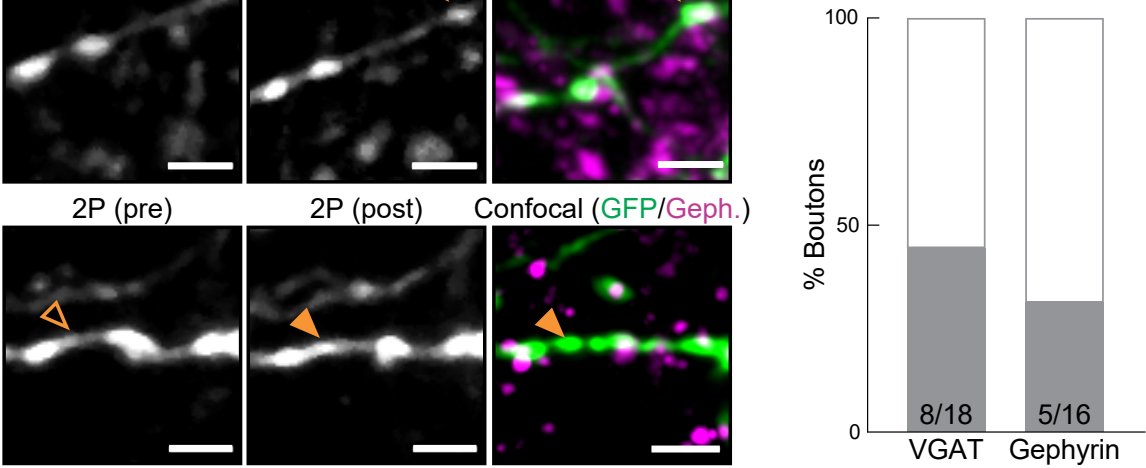

$J$

K

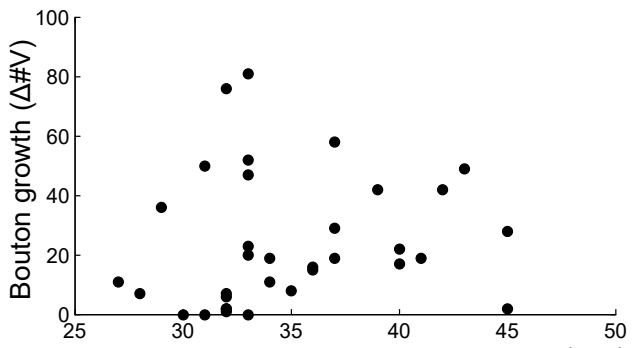

Time between break-in and glu uncaging $(\mathrm{min})$

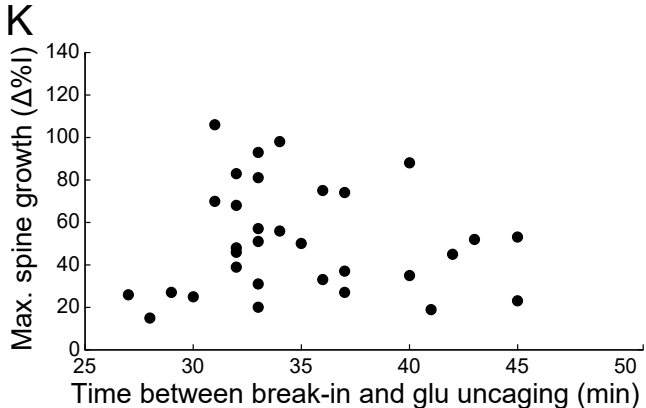


$\triangle$ Figure SI, related to Figure I: Bouton growth is independent of quantification method, threshold and time after break-in.

A,B: As an alternative measure of bouton volume, we determined the relative increase in integrated intensity $(\Delta \% \mathrm{l})$ of the inhibitory axon at the crossing compared to its baseline intensity, a measure which is often used to quantify growth of small structures such as spines (Harward et al., 20I6; Oh et al., 20I5). Heat maps showing bouton intensity $(\Delta \% \mathrm{I})$ over time of all experiments in non-stimulated $(\mathrm{A})$ and stimulated $(\mathrm{B})$ conditions. Each row represents a single experiment. The vertical white line indicates the end of the baseline period (first 20 minutes). White squares indicate missing time points. Experiments are sorted by maximal bouton growth.

C: Bouton growth occurrence over time in experiments in stimulated and non-stimulated conditions. Blue line indicates timepoints where bouton growth was different compared to baseline, as tested by Cochran's $Q$ test followed by post-hoc McNemar's test. *, $p<0.05$.

D: Scatter plot of absolute bouton volume growth $(\Delta \# \mathrm{~V})$ against relative bouton intensity growth $(\Delta \% \mathrm{l})$ from experiments without (black) and with (blue) spine stimulation. The two measures of bouton growth are highly correlated. Pearson's $r=0.82$ ( $p<10^{-18}$, Spearman).

E,F: Bouton growth occurrence plotted against growth threshold ( $\triangle \# \mathrm{~V}$ in $\mathrm{E}$ and $\Delta \% \mathrm{l}$ in F) for No Stim (black), Stim (blue), Mock (gray), NMDAR Block (yellow) and $\mathrm{Mg}^{2+}$ free (red) experiments. These graphs indicate that our conclusions do not depend on the choice of this threshold.

G,H: Left: Two-photon microscopy images showing two examples of a GFP-labeled inhibitory axon before ('pre') and after ('post') inhibitory bouton growth (without stimulation). Right: the same region is shown after fixation and staining against $(G)$ presynaptic vesicular GABA transporter (VGAT, magenta) and $(\mathrm{H})$ postsynaptic scaffold protein gephyrin (magenta). Arrowhead highlights axonal location where bouton growth was observed. Scale bars, $2 \mu \mathrm{m}$. I: Percentage of boutons positive (gray) and negative (white) for VGAT and gephyrin. Only inhibitory boutons that had grown in the last 60 minutes of the live imaging period were selected (see methods for details).

J,K: Bouton growth $(\mathrm{J})$ and maximal spine growth $(\mathrm{K})$ plotted against the time between whole-cell break-in and glutamate uncaging for all 'Stim' experiments (Fig. IM). These graphs suggest that washout is not a major factor in our experiments.

Data in G-I is reanalyzed from Figure I in (Frias et al., 2019).

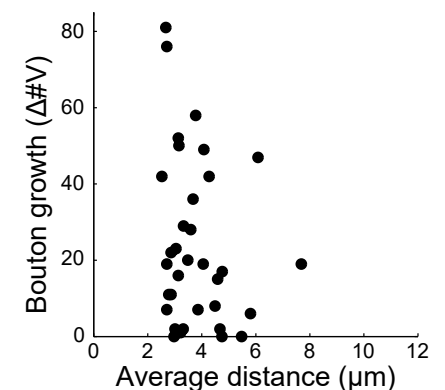

between unc. spots and crossing

Figure S2, related to Figure 2: Bouton growth is not correlated with distance to uncaging spot.

Scatter plot of bouton growth versus the average distance between the axon-dendrite crossing and the four uncaging spots. $\mathrm{p}=0.2 \mathrm{I}$, Spearman. 
A
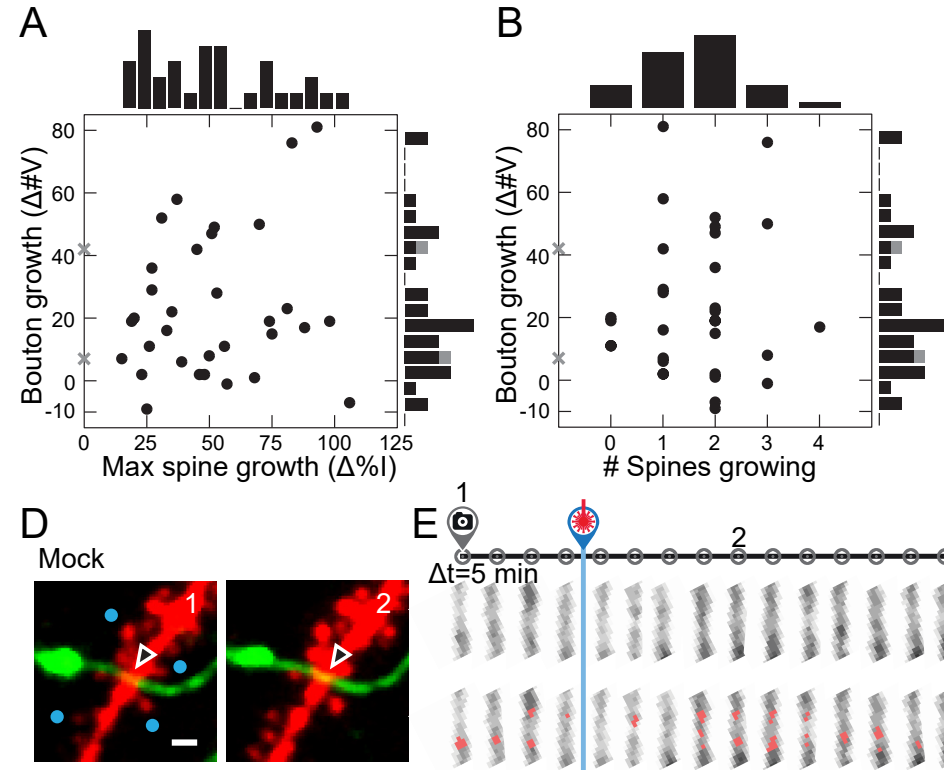

$E \stackrel{1}{0}$

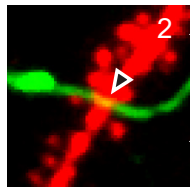

G

NMDAR Block

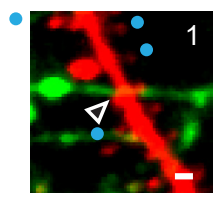

$\mathrm{J}_{\mathrm{Mg}^{2+} \text { Free }}$
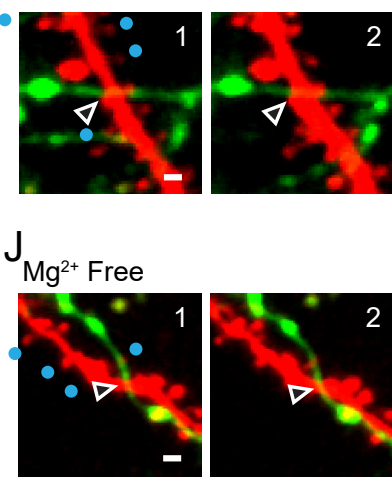

$\mathrm{H}$

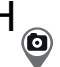

․

$\Delta t=5 \mathrm{~min}$

$\Delta t=5$ min $-\infty \cdots \cdots-\infty-\cdots$ miminmm

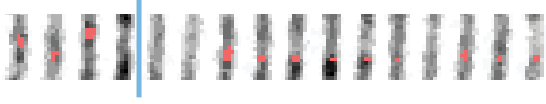

$\mathrm{K}$
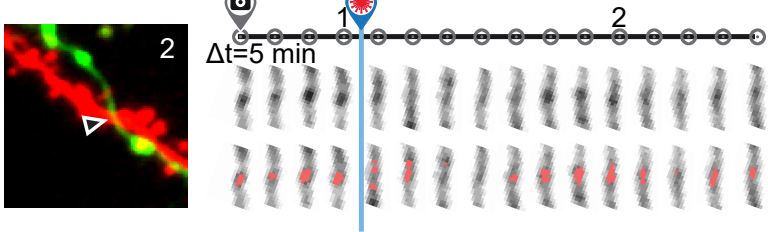

C Mock

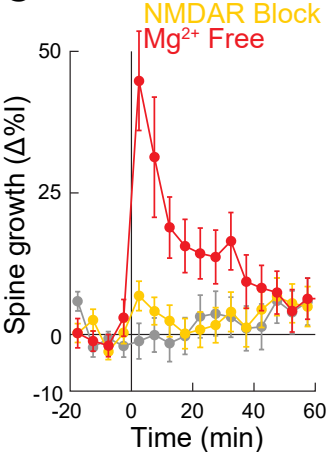

F
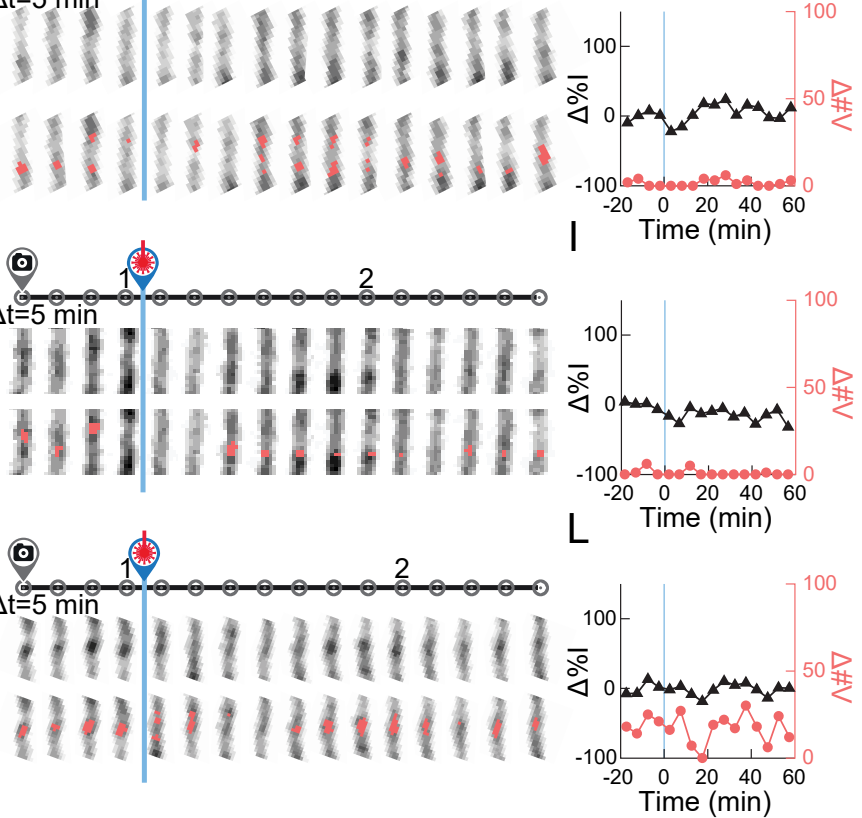

Figure S3, related to Figure 3: Bouton growth and spine growth are not correlated.

A,B: Scatter plot of maximal spine growth (A) or number of growing spines (B) against bouton growth per experiment. No correlations were found $(p=0.54(A)$ and $p=0.61(B)$, Spearman). Gray crosses indicate two experiments in which we could not determine spine volumes. Distributions are shown as histograms next to the scatter plot.

C: Average spine growth over time after spine stimulation in the absence of DNI-glutamate ('Mock', gray), when NMDA receptors were blocked with $50 \mu$ MAPV ('NMDAR block', yellow), and in $\mathrm{Mg}^{2+}$ free ACSF + $0.5 \mu$ MTTX ('Mger free', red).

D-F: Example of an experiment in which spine stimulation and postsynaptic depolarization were applied in absence of DNI-glutamate ('Mock'). D: Images of two time points indicated in E. Arrowheads point towards the axon crossing, blue dots indicate uncaging locations. Scale bar is I $\mu \mathrm{m}$. E: Axonal segment at the crossing is displayed for all time points. The voxels above axon threshold are indicated in pink in the lower panel. F: Quantification of bouton volume over time, measured as the increase in number of voxels above axon threshold $(\Delta \# \mathrm{~V}$, pink) and increase in relative intensity $(\Delta \%$, black).

G-I: As D-F, example of an experiment in which spine stimulation was performed in presence of the NMDAR antagonist $50 \mu \mathrm{MAPV}$.

J-L: As in D-F, example of an experiment in which spine stimulation was performed without depolarization in absence of extracellular $\mathrm{Mg}^{2+}$ and in presence of $0.5 \mu \mathrm{MTTX}$. 
GFP-labeled

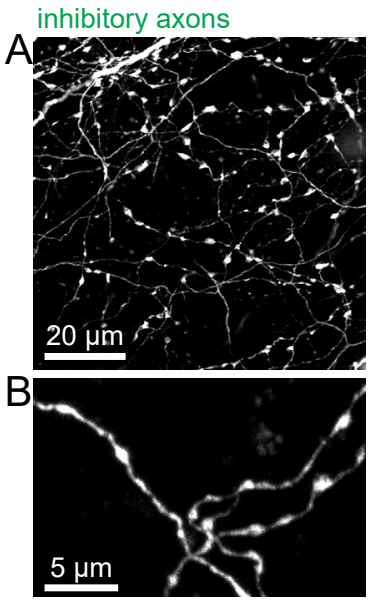

$\mathrm{D}_{\mathrm{AM} 251}$

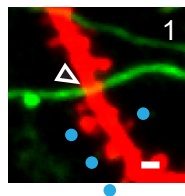

$\mathrm{G}_{\mathrm{TH}}$

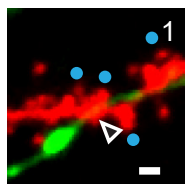

$\mathrm{J}_{\mathrm{JZL} 184}$
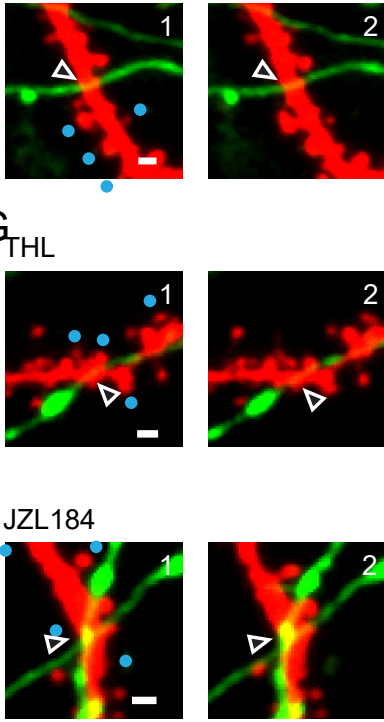

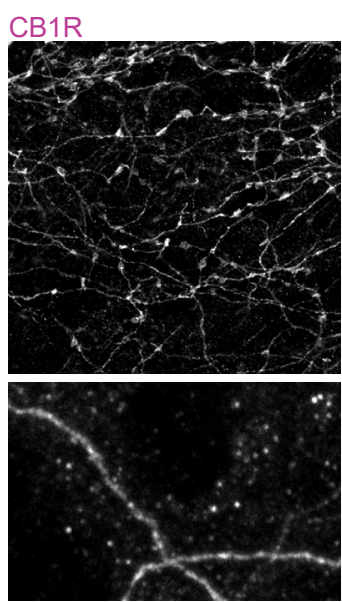

$\mathrm{E}$
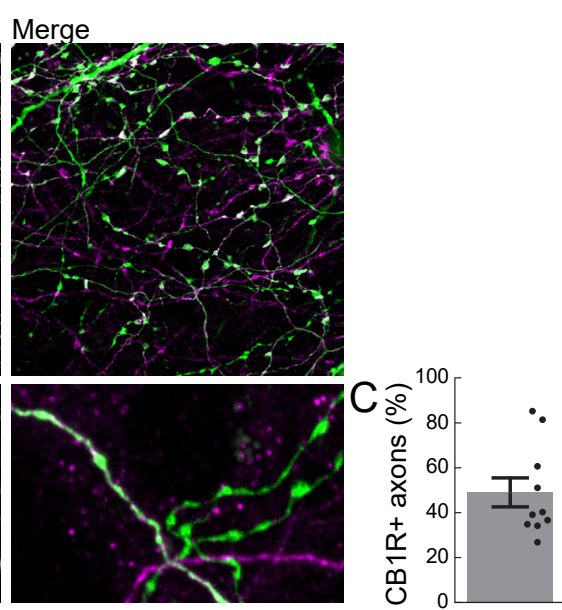

$\mathrm{F}$

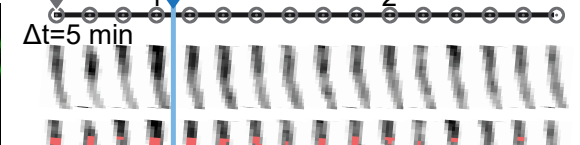
IIIIIIIIIIIIIII
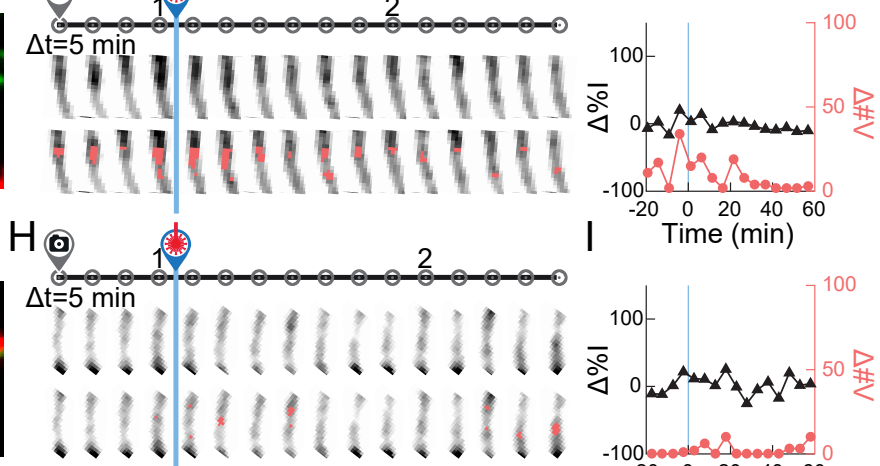

Figure S4, related to Figure 4:CBI receptor expression, example axon-dendrite crossings and local BDNF application. A: Example of immunohistochemistry against the $C B I$ receptor (CBIR). Left panel: GFP-positive axons in GAD65GFP slices. Middle panel: $\mathrm{CBI}$ receptors. Right panel:merged image. In accordance with the reported low expression levels at postsynaptic dendrites and spines and glia cells (Kano et al., 2009), we did not detect substantial CBIR immunostaining in non-axonal structures.

B: As in A, example showing CBIR-positive and CBIR-negative GFP-labeled axons and CBIR-positive unlabeled axon.

C: Quantification of the percentage of GFP-labeled axons positive for CBIR. Bar represents mean \pm SEM $(n=10$ image stacks, $\mathrm{N}=3$ slices). Dots represent individual image stacks.

D-F: Example of an experiment in which spine stimulation was performed in presence of the CBIR antagonist AM25I $(5 \mu$ M). D: Images of two time points indicated in E.Arrowheads point towards the axon crossing, blue dots indicate uncaging locations. Scale bar is I $\mu \mathrm{m}$. E: Axonal segment at the crossing is displayed for all time points. The voxels above axon threshold are indicated in pink in the lower panel. F: Quantification of bouton volume over time, measured as the increase in number of voxels above axon threshold $(\triangle \# \mathrm{Z}$, pink) and increase in relative intensity 


\section{Chapter 3}

$(\Delta \%$ l, black).

G-I: As D-F, example of an experiment in which spine stimulation was performed with $5 \mu \mathrm{MTHL}$ in the patch pipette. THL is a lipase inhibitor with high affinity for the 2-AG synthesis enzyme DAGL.

J-L: As in D-F, example of an experiment in which spine stimulation was performed in presence of $100 \mathrm{nM} \mathrm{JZLI84,}$ an inhibitor of the 2-AG degradation enzyme MAGL. 


\section{References}

Bloss, E.B., Cembrowski, M.S., Karsh, B., Colonell, J., Fetter, R.D., and Spruston, N. (20I6). Structured dendritic inhibition supports branch-selective integration in CAI pyramidal cells. Neuron 89, 1016-1030

Bourne, J.N., and Harris, K.M. (20II). Coordination of size and number of excitatory and inhibitory synapses results in a balanced structural plasticity along mature hippocampal CAI dendrites during LTP. Hippocampus 2I, 354-373.

Branco, T., and Häusser, M. (20I0). The single dendritic branch as a fundamental functional unit in the nervous system. Curr. Opin. Neurobiol. 20, 494502.

Castillo, P.E., Younts, T.J., Chávez, A.E., and Hashimotodani, Y. (20I2). Endocannabinoid signaling and synaptic function. Neuron 76,70 81 .

Chen, J.L., Villa, K.L., Cha, J.W., So, P.T.C., Kubota, Y., and Nedivi, E. (20/2). Clustered dynamics of inhibitory synapses and dendritic spines in the adult neocortex. Neuron 74, 36I-373.

Chen, S.X., Kim, A.N., Peters, A.J., and Komiyama, T. (20I5). Subtype-specific plasticity of inhibitory circuits in motor cortex during motor learning. Nat. Neurosci. I8, I 109-I I I5.

Chiu, C.Q., Martenson, J.S., Yamazaki, M., Natsume, R., Sakimura, K., Tomita, S., Tavalin, S.J., and Higley, M.J. (20I8). Input-specific NMDAR-dependent potentiation of dendritic GABAergic inhibition. Neuron 97, 368-377.e3

Cui,Y., Prokin, I., Xu, H., Delord, B., Genet, S.,Venance, L., and Berry, H. (20I6). Endocannabinoid dynamics gate spike- timing dependent depression and potentiation. Elife $5, \mathrm{I}-32$

Dobie, F.A., and Craig, A.M. (201I). Inhibitory synapse dynamics: coordinated presynaptic and postsynaptic mobility and the major contribution of recycled vesicles to new synapse formation. J. Neurosci. 3 I, I048I-10493.

Dudok, B., Barna, L., Ledri, M., Szabó, S.I., Szabadits, E., Pintér, B., Woodhams, S.G., Henstridge, C.M., Balla, G.Y., Nyilas, R., et al. (20I5). Cell-specific STORM super-resolution imaging reveals nanoscale organization of cannabinoid signaling. Nat. Neurosci. 18, 75-86.
Flores, C.E., Nikonenko, I., Mendez, P., Fritschy, J.-M., Tyagarajan, S.K., and Muller, D. (20I5). Activitydependent inhibitory synapse remodeling through gephyrin phosphorylation. Proc. Natl. Acad. Sci. I/ 2, E65-E72.

Frias, C.P., Liang, J., Bresser, T., Scheefhals, L., Kesteren, M. van, Dorland, R. van, Hu, H.Y., Bodzeta, A., Van Bergen en Henegouwen, P.M.P., Hoogenraad, C.C., et al. (2019). Semaphorin4D induces inhibitory synapse formation by rapid stabilization of presynaptic boutons via MET coactivation. J. Neurosci. in press.

Froemke, R.C. (20I5). Plasticity of cortical excitatoryinhibitory balance. Annu. Rev. Neurosci. 38, 195-219.

Gambino, F., Pagès, S., Kehayas, V., Baptista, D., Tatti, R., Carleton, A., and Holtmaat, A. (20I4). Sensoryevoked LTP driven by dendritic plateau potentials in vivo. Nature $5 / 5,116-119$

Govindarajan, A., Israely, I., Huang, S.-Y., and Tonegawa, S. (20II). The dendritic branch is the preferred integrative unit for protein synthesis-dependent LTP. Neuron 69, I32-146.

Harnett, M.T., Makara, J.K., Spruston, N., Kath, W.L., and Magee, J.C. (20I2). Synaptic amplification by dendritic spines enhances input cooperativity. Nature 49I, 599-602.

Hartzell,A.L., Martyniuk, K.M., Brigidi, G.S., Heinz, D.A., Djaja, N.A., Payne,A., and Bloodgood, B.L. (20I8). NPAS4 recruits CCK basket cell synapses and enhances cannabinoid-sensitive inhibition in the mouse hippocampus. Elife 7, I-24.

Harvey, C.D., and Svoboda, K. (2007). Locally dynamic synaptic learning rules in pyramidal neuron dendrites. Nature 450, I 195-1200.

Harward, S.C., Hedrick, N.G., Hall, C.E., Parra-Bueno, P., Milner, T.A., Pan, E., Laviv, T., Hempstead, B.L., and Yasuda, R. (2016). Autocrine BDNF-TrkB signalling within a single dendritic spine. Nature 538, 99-103.

Hashimotodani, Y., Ohno-shosaku, T., and Kano, M. (2007). Ca2+-assisted receptor-driven endocannabinoid release: mechanisms that associate presynaptic and postsynaptic activities. Curr. Opin. Neurobiol. 17, 360-365.

lacaruso, M.F., Gasler, I.T., and Hofer, S.B. (2017). Synaptic organization of visual space in primary visual cortex. Nature 547, 449-452. 
Jadi, M., Polsky, A., Schiller, J., and Mel, B.W. (20/2). Location-dependent effects of inhibition on local spiking in pyramidal neuron dendrites. PLoS Comput. Biol. 8, el 002550.

Jung, K.-M., Sepers, M., Henstridge, C.M., Lassalle, O., Neuhofer, D., Martin, H., Ginger, M., Frick, A., DiPatrizio, N. V., Mackie, K., et al. (20/2). Uncoupling of the endocannabinoid signalling complex in a mouse model of fragile $X$ syndrome. Nat. Commun. 3, 1080.

Kano, M., Ohno-Shosaku, T., Hashimotodani, Y., and Watanabe, M.U.M. (2009). Endocannabinoidmediated control of synaptic transmission. Physiol. Rev. 89, 309-380.

Keck, T., Scheuss, V., Jacobsen, R.I.,Wierenga, C.J., Eysel, U.T., Bonhoeffer, T., and Hübener, M. (20I I). Loss of sensory input causes rapid structural changes of inhibitory neurons in adult mouse visual cortex. Neuron 7I, 869-882.

Krueger-Burg, D., Papadopoulos, T., and Brose, N. (2017). Organizers of inhibitory synapses come of age. Curr. Opin. Neurobiol. 45, 66-77.

López-Bendito, G., Sturgess, K., Erdélyi, F., Szabó, G. Molnár, Z., and Paulsen, O. (2004). Preferential origin and layer destination of GAD65-GFP cortical interneurons. Cereb. Cortex 14, II22II33.

Losonczy, A., and Magee, J.C. (2006). Integrative properties of radial oblique dendrites in hippocampal CAI pyramidal neurons. Neuron $50,29 \mid-307$

Lovett-Barron, M., Turi, G.F., Kaifosh, P., Lee, P.H., Bolze, F., Sun, X.-H., Nicoud, J.-F., Zemelman, B. V, Sternson, S.M., and Losonczy, A. (20I2). Regulation of neuronal input transformations by tunable dendritic inhibition. Nat. Neurosci. 15, 423-430.

Lovett-Barron, M., Kaifosh, P., Kheirbek, M.A., Danielson, N., Zarembla, J.D., Reardon, T.R., Turi, G.F., Hen, R., Zemelman, B. V, and Losonczy, A. (20I4). Dendritic inhibition in the hippocampus supports fear learning. Science 343, 857-863.

Megías, M., Emri, Z., Freund, T.F., Gulyás, A.I., and Megias, M. (200I). Total number and distribution of inhibitory and excitatory synapses on hippocampal CAI pyramidal cells. Neuroscience 102, 527-540.

Monday, H.R., and Castillo, P.E. (20I7). Closing the gap: long-term presynaptic plasticity in brain function and disease. Curr. Opin. Neurobiol. 45, I06-II2.

Müllner, F.E., Wierenga, C.J., and Bonhoeffer, T. (20I5). Precision of inhibition: dendritic inhibition by individual GABAergic synapses on hippocampal pyramidal cells is confined in space and time. Neuron 87, 576-589.

Oh, W.C., Parajuli, L.K., and Zito, K. (2015). Heterosynaptic Structural Plasticity on Local Dendritic Segments of Hippocampal CAI Neurons. Cell Rep. 10, 162-169.

Petrini, E.M., Ravasenga,T., Hausrat,T..., lurilli, G., Olcese, U., Racine, V., Sibarita, J.-B., Jacob, T.C., Moss, S.J., Benfenati, F., et al. (20/4). Synaptic recruitment of gephyrin regulates surface GABA receptor dynamics for the expression of inhibitory LTP. Nat. Commun. 5, 392I.

Piomelli, D. (20|4). More surprises lying ahead. The endocannabinoids keep us guessing. Neuropharmacology 76, 228-234.

Poirazi, P., and Mel, B.W. (200I). Impact of active dendrites and structural plasticity on the memory capacity of neural tissue. Neuron 29, 779-796.

Schuemann, A., Klawiter, A., Bonhoeffer, T., and Wierenga, C.J. (20I3). Structural plasticity of GABAergic axons is regulated by network activity and $G_{A B A}$ receptor activation. Front. Neural Circuits 7, I-I6.

Schulz, J.M., Knoflach, F., Hernandez, M.C., and Bischofberger, J. (2018). Dendrite-targeting interneurons control synaptic NMDA-receptor activation via nonlinear $\alpha 5-G A B A_{A}$ receptors. Nat. Commun. 9, 3576.

De Simoni, A., Griesinger, C.B., and Edwards, F.A. (2003). Development of rat CAI neurones in acute versus organotypic slices: role of experience in synaptic morphology and activity.J. Physiol. 550, I35-147.

Stoppini, L., Buchs, P.A., and Muller, D. (I99|).A simple method for organotypic cultures of nervous tissue. J. Neurosci. Methods 37, I73-182.

Stuart, G., Spruston, N., Sakmann, B., and Häusser, M. (1997). Action potential initiation and backpropagation in neurons. Trends Neurosci. 20, |25-|3|.

Tanaka, J., Horiike, Y., Matsuzaki, M., Miyazaki, T., EllisDavies, G.C.R., and Kasai, H. (2008). Protein synthesis and neurotrophin-dependent 
structural plasticity of single dendritic spines. Science 319, 1683-1687.

Tønnesen, J., Katona, G., Rózsa, B., and Nägerl, U.V. (20I4). Spine neck plasticity regulates compartmentalization of synapses. Nat. Neurosci. 17, 678-685.

Villa, K.L., Berry, K.P., Subramanian, J., Cha, J.W., Oh, W.C., Kwon, H.-B., Kubota, Y., So, P.T.C., and Nedivi, E. (20I6). Inhibitory synapses are repeatedly assembled and removed at persistent sites in vivo. Neuron 89, 756-769.

Wang, W., Jia, Y., Pham, D.T., Palmer, L.C., Jung, K.-M., Cox, C.D., Rumbaugh, G., Piomelli, D., Gall, C.M., and Lynch, G. (2017). Atypical endocannabinoid signaling initiates a new form of memory-related plasticity at a cortical input to hippocampus. Cereb. Cortex I-I4.

Weber, J.P., Andrásfalvy, B.K., Polito, M., Magó, Á., Ujfalussy, B.B., and Makara, J.K. (20I6). Locationdependent synaptic plasticity rules by dendritic spine cooperativity. Nat. Commun. 7, I I 380.

Wierenga, C.J., Becker, N., and Bonhoeffer, T. (2008). GABAergic synapses are formed without the involvement of dendritic protrusions. Nat. Neurosci. I I, 1044-1052.

Wierenga, C.J., Müllner, F.E., Rinke, I., Keck, T., Stein, V., and Bonhoeffer, T. (20I0). Molecular and electrophysiological characterization of GFPexpressing CAI interneurons in GAD65-GFP mice. PLoS One 5, el59I5.

Wilson, D.E.,Whitney, D.E., Scholl, B., and Fitzpatrick, D. (20I6). Orientation selectivity and the functional clustering of synaptic inputs in primary visual cortex. Nat. Neurosci. 19, 1003-1009.

Wilson, D.E., Scholl, B., and Fitzpatrick, D. (20I8). Differential tuning of excitation and inhibition shapes direction selectivity in ferret visual cortex. Nature 560, 97-I0I.

Younts, T.J., Chevaleyre, V., and Castillo, P.E. (20I3). CAI pyramidal cell theta-burst firing triggers endocannabinoid-mediated long-term depression at both somatic and dendritic inhibitory synapses. J. Neurosci. 33, I 3743-1 3757. 


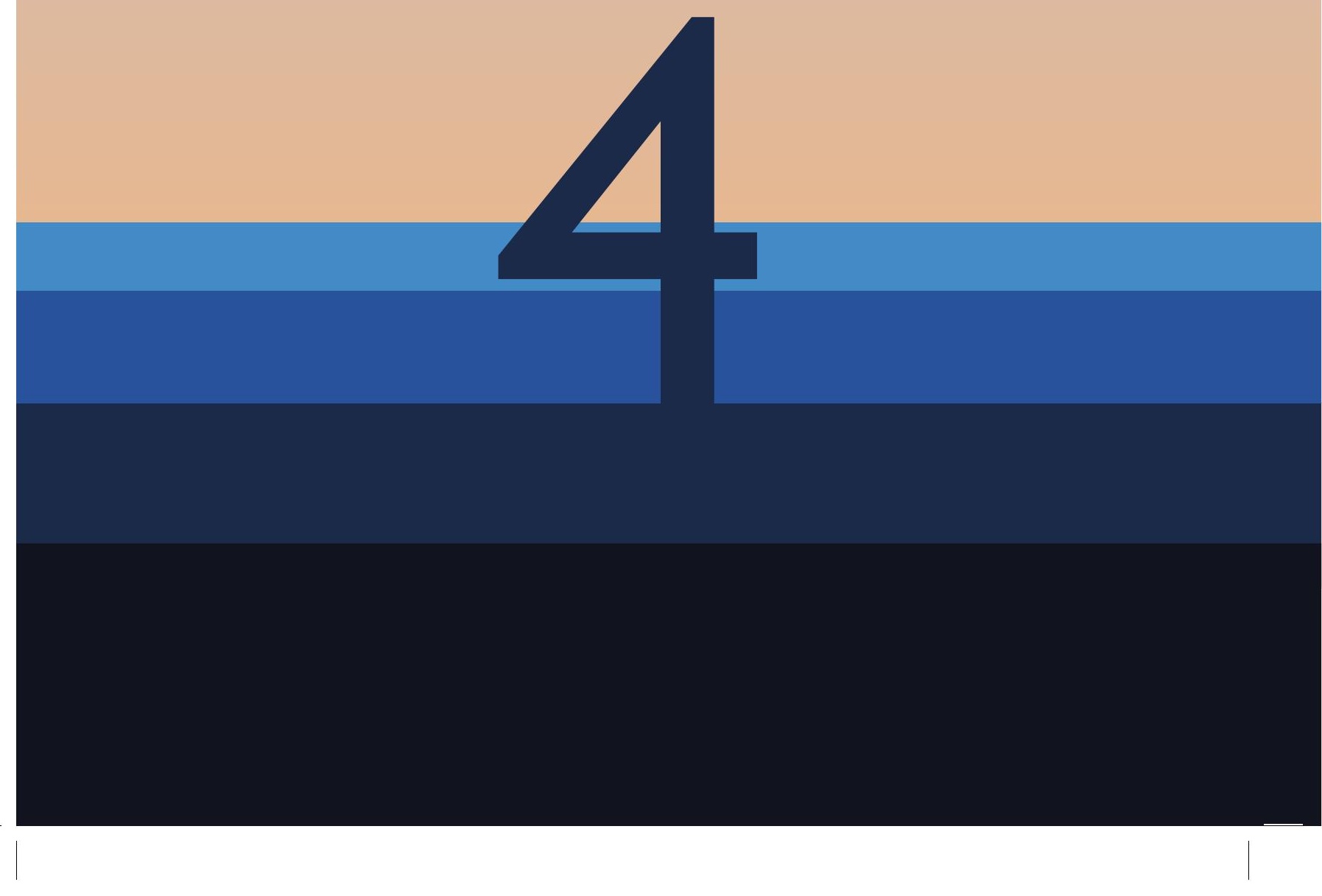




\section{Endocannabinoid signaling in inhibitory synapse dynamics and excitatory synaptic plasticity}

Dennis L. H. Kruijssen, Iris G. L. M. Rosmalen, Jian Liang, Corette J.Wierenga

Department of Biology, Science for Life, Utrecht University, Utrecht, The Netherlands

The data presented in Figure I was published as part of Hu \& Kruijssen et al., Cell Reports 27, (2019). The other figures in this chapter show unpublished preliminary data. 


\section{Abstract}

Endocannabinoids play an important role in mediating heterosynaptic depression of inhibitory synapses, but more recently atypical roles for endocannabinoids have emerged. In chapter 3, we described a mechanism where inhibitory bouton growth on a CAI pyramidal dendrite is triggered by activation of excitatory synapses on the dendrite, and this process is mediated by the endocannabinoid 2-AG. Here, we present preliminary data on the effect of 2-AG on inhibitory bouton dynamics and inhibitory synapse formation. We find that brief exposure of inhibitory axons to 2-AG leads to a bias towards bouton growth and repeated brief treatments lead to an increase of mIPSC frequency 24h later, in agreement with the formation of new inhibitory synapses. Prolonged exposure to 2-AG however leads to a decrease in bouton density and the number of inhibitory synapses, but also seems to affect the expression level of the $\mathrm{CBI}$ receptor. We furthermore show that manipulations of the endocannabinoid system affects structural LTP of dendritic spines upon two-photon glutamate uncaging. Together, these data suggest that endocannabinoid signaling might play important and unexpected roles in inhibitory synapse dynamics and excitatory synaptic plasticity. 


\section{Introduction}

The endocannabinoid (eCB) system is an important system of signaling molecules and receptors in the nervous system. During development, eCB signaling is important for processes such as cell migration, neurite outgrowth, and axon guidance (Gaffuri et al., 20 I2; Harkany et al., 2007). In mature neurons, eCBs act as retrograde messengers, traveling from the postsynaptic to the presynaptic compartment, regulating the short- and long-term plasticity of synapses (Castillo et al., 2012; Kano et al., 2009; Lu and Mackie, 2016). In chapter 3 , we found that upon stimulation of a cluster of excitatory synapses, eCBs mediated the growth of an inhibitory presynaptic bouton on an axon-dendrite crossing nearby. Here, we further explore the unexpected role of eCB signaling in synapse formation and plasticity.

Endocannabinoids are a class of lipid-based signaling molecules. Typically, we consider two main endocannabinoids: 2-arachidonoylglycerol (2-AG) and anandamide (AEA). In the hippocampus, 2-AG is estimated to be present at over 200-fold higher concentrations than AEA (Bisogno et al., 1999; Buczynski and Parsons, 2010; Richardson et al., 2007). 2-AG is synthesized on demand in response to increases in intracellular $\mathrm{Ca}^{2+}$ concentration and metabotropic glutamate receptor activation. These two factors can activate phospholipase $C \beta(P L C \beta)$, which produces diacylglycerol (DAG). This precursor is used for the production of 2-AG by the calcium-sensitive enzyme diacylglycerol lipase (DAGL) (Hashimotodani et al., 2005; Heifets and Castillo, 2009; Jung et al., 2007; Murataeva et al., 20I4; Shonesy et al., 2015; Stella et al., 1997), which is enriched in dendritic spines (Katona et al., 2006; Yoshida et al., 2006). Synthesis of AEA is thought to occur via NAPE-PLD but is less well understood (Piomelli, 20I4).

After synthesis and release, endocannabinoids can activate endocannabinoid receptors. There are two classical eCB receptors: the $C B 1$ receptor and the $C B 2$ receptor, which are both G-protein coupled receptors. While the $C B 2$ receptor is mainly found on cells of the immune system (Cabral et al., 20I5), the CBI receptor can be found throughout the brain (Hu and Mackie, 2015; Kano et al., 2009). The CBI receptor is highly expressed in CCK-type inhibitory neuron axons and boutons (Dudok et al., 2015; Katona et al., 1999; Marsicano and Lutz, 1999; Nyíri et al., 2005; Tsou et al., 1999). CBI receptor expression is however not exclusive to GABAergic neurons, as it is thought that excitatory neurons (Katona et al., 2006; Kawamura et al., 2006; Marsicano and Lutz, 1999) and astrocytes (Navarrete et al., 20I4; Navarrete and Araque, 2008) express the CBI receptor at lower levels.

eCBs are well known for regulating several forms of plasticity at inhibitory synapses (Younts and Castillo, 20I4). On short time scales, eCBs play a role in depolarization-induced suppression of inhibition (DSI). Prolonged depolarization of the postsynaptic cell leads to the calcium-mediated release of endocannabinoids, that activate presynaptic $\mathrm{CBI}$ receptors in inhibitory axons. GABAergic synaptic transmission is suppressed by downregulation of the release probability (likely mediated by inhibition of presynaptic voltage-gated calcium channels) that lasts for tens of seconds (Castillo et al., 20I2; Ohno-Shosaku et al., 200I; Wilson and Nicoll, 200I). On longer time scales, inhibitory synapses can be affected by endocannabinoid-mediated long-term depression (eCB-LTD). Theta burst or tetanic stimulation of excitatory afferents leads to the production of endocannabinoids in the postsynaptic neuron (Chevaleyre and Castillo, 2003, 2004). The CBI receptor is activated for several minutes at the inhibitory presynapse and this leads to reduction of PKA activity and a longer lasting suppression of vesicle release (Castillo et al., 20I2; Chevaleyre et al., 
2007; Chevaleyre and Castillo, 2003). Induction of eCB-LTD likely also requires activation of the inhibitory presynapse (Heifets et al., 2008) and protein synthesis (Younts et al., 20I6). Similar eCB-mediated short- and long-term depression has been observed in excitatory presynapses throughout the brain (Kano et al., 2009; Kreitzer and Regehr, 200I; OhnoShosaku et al., 2002; Sjöström et al., 2003; Yasuda et al., 2008).

While eCBs are typically involved in weakening of synapses, recently atypical forms of eCB signaling have been reported where eCBs mediate long-term potentiation. High frequency stimulation of the lateral perforant path, one of the cortical inputs into the hippocampus, leads to 2-AG-mediated and presynaptically expressed LTP of excitatory synapses onto dentate granule cells. This form of LTP requires actin remodeling in the presynaptic neuron (Wang et al., 2016). Low frequency paired stimulation of the perforant path and the Schaeffer collaterals leads to 2-AG mediated LTP of the Schaeffer collateral-CAI synapse specifically (Xu et al., 2012). At corticostriatal synapses, it has been shown that eCB-mediated spiketiming dependent LTP and LTD can occur (Cui et al., 20I5, 20I6). The polarity of plasticity seems to be dependent on the level and duration of eCB release (Cui et al., 2016). These results demonstrate that $\mathrm{eCBs}$ do not just mediate synaptic depression but are involved in many other processes, suggesting that the eCB system is highly complex and not yet fully understood.

Inhibitory synapses are highly dynamic structures that can appear, disappear and reappear on a wide range of time scales (Chen et al., 2012; Frias et al., 2019; Schuemann et al., 20I3; Villa et al., 2016; Wierenga et al., 2008). These dynamics are part of a trial-and-error process of inhibitory synapse formation and maturation and may play a role in coordinating excitation and inhibition (see chapter I and (Frias and Wierenga, 20I3; Wierenga, 20I7)). Inhibitory bouton formation is thought to be the first step in inhibitory synapse formation, but the mechanisms that underly the formation and maturation of an inhibitory synapse are not yet fully understood. We therefore decided to further explore the role of eCB signaling in inhibitory bouton growth and synapse formation. In this chapter, we present a collection of preliminary data regarding this topic. We show that brief and local application of the endocannabinoid 2-AG can promote bouton growth. We used electrophysiological recordings to show that brief 2-AG treatments lead to an increase in miniature IPSC frequency $24 \mathrm{~h}$ later, in agreement with an increase in the number of functional inhibitory synapses. These data suggest that endocannabinoid signaling can promote inhibitory bouton formation and growth on short time scales, which eventually leads to an increase of functional inhibitory synapses on longer time scales. However, prolonged exposure to 2-AG for 48 hour leads to a reduction of inhibitory synapse number, which might be caused by desensitization and internalization of the $\mathrm{CBI}$ receptor. Additionally, we look into the relation between $\mathrm{eCB}$ signaling and excitatory synaptic plasticity. We present data suggesting that manipulations of the endocannabinoid system affect structural plasticity of individual dendritic spines upon glutamate uncaging. Together our results suggest that eCB signaling is involved in synapse formation and plasticity at both excitatory and inhibitory synapses. 


\section{Results}

In chapter 3, we stimulated four excitatory synapses on a dendrite for one minute using two-photon glutamate uncaging. This causes the dendrite to produce endocannabinoids, that act as retrograde messengers and induce the growth of a inhibitory bouton. Since the classical triggers of endocannabinoid production (an increase in calcium concentration and metabotropic glutamate receptor activation) are likely restricted in space and increased specifically during the glutamate uncaging stimulus, we postulate that endocannabinoid release is mostly limited to the duration of spine stimulation. We therefore asked if brief and local application of endocannabinoids is sufficient to induce the growth of inhibitory boutons.

To locally apply the endocannabinoid 2-AG onto multiple axons, we placed a glass pipette containing $100 \mu \mathrm{M} 2-\mathrm{AG}$ or a control solution in the top layer of an organotypic hippocampal slice of GAD65-GFP transgenic mice, which express GFP in a subset of inhibitory neurons (López-Bendito et al., 2004; Wierenga et al., 2010) (Figure IA, left). By applying pressure to the pipette, we were able to locally release $2-A G$ in a controlled manner. The pipette also contained Alexa568, enabling us to estimate the area exposed to the pipette contents (Figure IA, right).After acquiring two-photon images every 5 minutes for a baseline period of 5 time points, 2-AG or control solution was applied for I minute in $0.5 \mathrm{~Hz}$ pulses (see Methods for details). As eCB level and duration of eCB release can play a crucial role in signaling (Cui et al., 2016), we designed the local application protocol to mimic endocannabinoid release as it would occur upon the glutamate uncaging stimulation that we used in chapter 3. After local application, we continued to acquire images every 5 minutes for up to an hour (Figure IB).

To assess the dynamics of inhibitory presynaptic boutons, we classified boutons based on their dynamic behavior before and after local application (Figure IC, see Methods for details). We then quantified the density of each bouton class for multiple axons (Figure ID). The bouton dynamics observed in these experiments are in general agreement with our previous work (Frias et al., 2019). We do observe a smaller fraction of persistent boutons than before (54\% persistent boutons, while Frias et al. reports $77 \%$ ). This increased level of dynamics might be explained by the shorter image acquisition interval. Additionally, the mechanical stress of puffing could increase dynamics.

Upon 2-AG application, we observe a small trend towards a higher density of new boutons ( $\mathrm{N}$, absent before treatment, sometimes present after treatment). than under control conditions (Figure ID,E, $\mathrm{p}=0.56, \mathrm{MW}$ ). The trend towards more new boutons could imply that exposing axons locally and briefly to 2-AG results in a bias towards more boutons. Surprisingly, while the density of lost (L), stabilizing (S), and destabilizing (D) boutons is the same between control and 2-AG conditions, a significant increase of intermittent boutons (I, sometimes present both before and after treatment) is observed upon 2-AG application (Figure ID,F, $\mathrm{p}=0.04, \mathrm{MW}$ ). This could explained by an overall increase of dynamics in the 2 -AG condition $(54 \pm 3 \%$ persistent boutons in Control versus $45 \pm 4 \%$ in $2-A G$; $p=0.04, M W$; data not shown). This increase in dynamics is mostly picked up by the largest non-persistent category, the intermittent boutons.

While classifying boutons allows quantifying bouton dynamics in terms of absence or presence, it does not take into consideration the size of a bouton. Bouton size is thought to be correlated with the size of the postsynapse and with synaptic strength (Cheetham et 

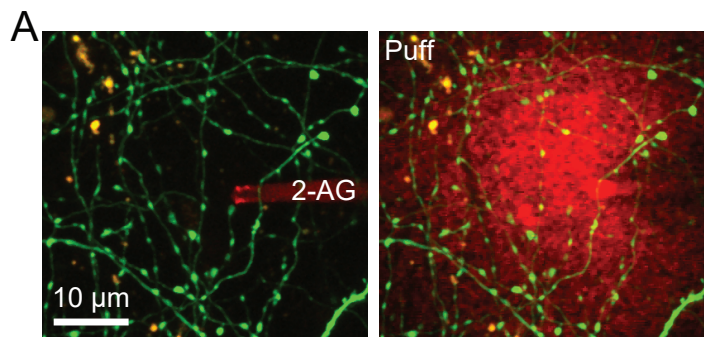

B

- Persistent bouton

- Non-persistent bouton

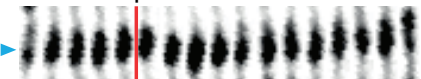

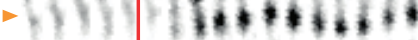

$-6160010,600060$

Time Puff $\Delta \mathrm{t}=5 \mathrm{~min}$
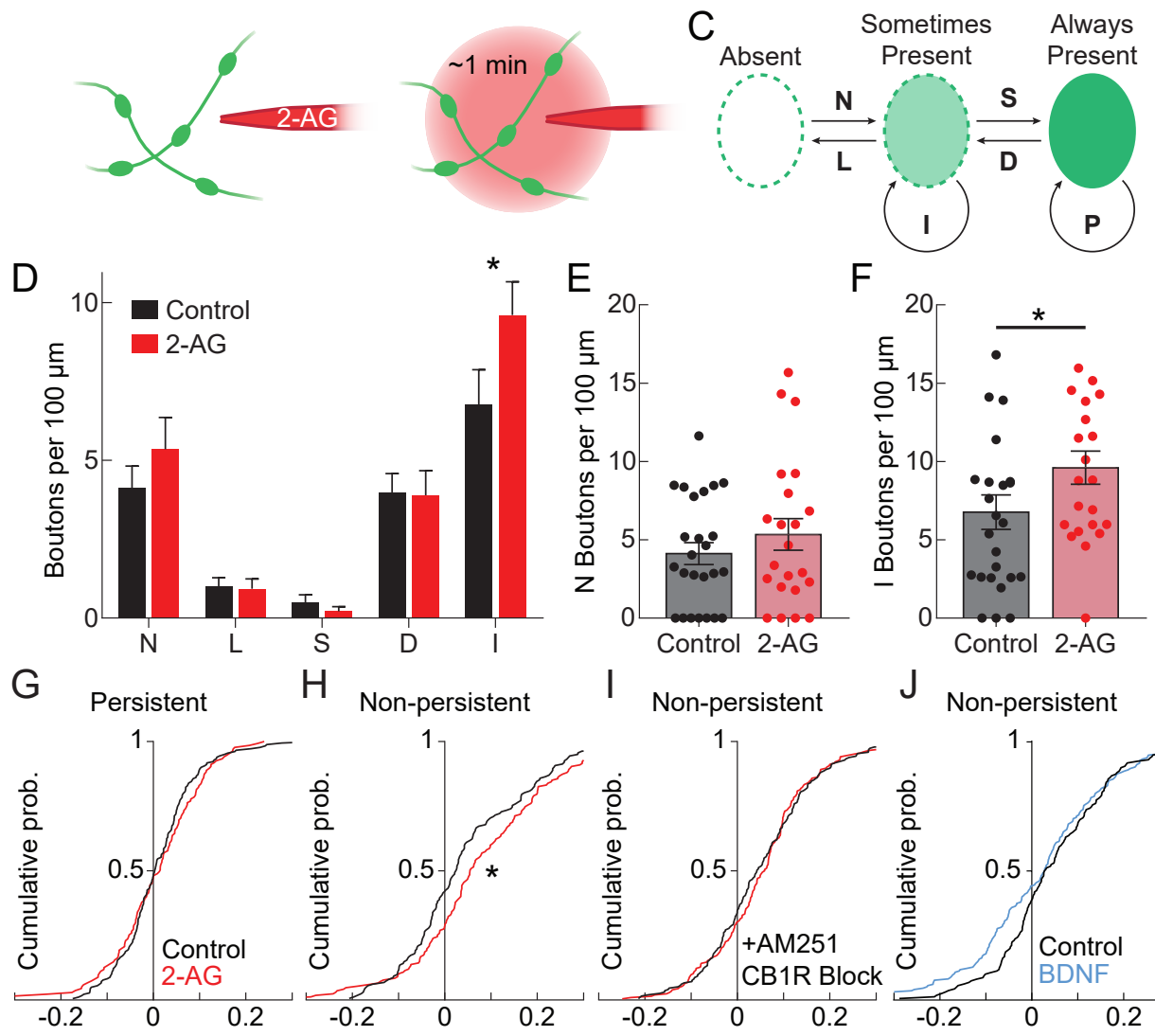

$\mathrm{H}$ Non-persistent

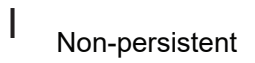

$J$ Non-persistent
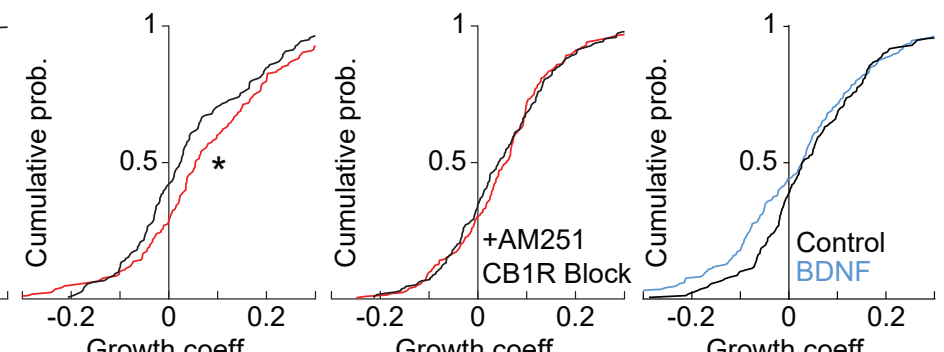

$\mathrm{K}$

$\mathrm{L}$

Growth coeff.

Growth coeff.
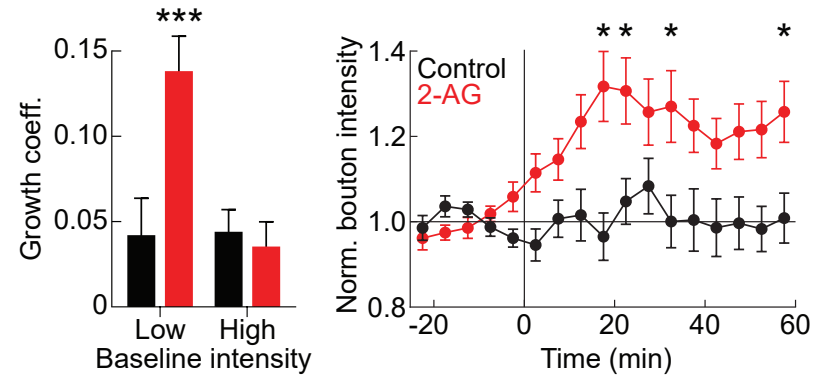
$\leq$ Figure I. local application of 2-AG promotes bouton growth.

A: Example images of a local application experiment before (top left) and during (top right, 'puff') local application. $100 \mu \mathrm{M}$ 2-AG (and Alexa568 for visualization, red) was applied via a pipette close to inhibitory axons (green). Bottom: schematic representation of the experimental setup.

B: An axonal stretch (straightened) is shown over time. Imaged are taken every 5 minutes. After 5 time points, the local application stimulus ("puff") is applied. Blue arrowheads indicate persistent boutons (present in all time points), while the orange arrowhead indicates a non-persistent bouton (present in at least two, but not all, time points).

C: Schematic representation of the categories used for the classification of bouton dynamics. The imaging period is divided in two periods: before treatment and after treatment. Boutons can be completely absent, sometimes present, or always present during a period. The arrows indicate a change in behavior from the first to the second period. New $(\mathrm{N})$ boutons are absent before and sometimes present after treatment. Lost (L) boutons are sometimes present before and absent after treatment. Stabilizing (S) boutons are sometimes present before and always present after treatment. Destabilizing $(D)$ boutons are always present before and sometimes present after treatment. Intermittent (I) boutons are sometimes present before and after treatment. Persistent $(\mathrm{P})$ boutons are always present before and after treatment.

D: Bouton density of the different nonpersistent bouton categories for control (black; $n=25$ axons, $N=9$ ) and 2-AG (red; $n=22$ axons, $N=8$ ) treated axons. Bars represent mean \pm SEM. *, $p<0.05$, Mann Whitney test.

E: Density of new boutons for control and 2-AG treated axons. Bars represent mean \pm SEM. Dots represent individual axons. New boutons density is slightly increased after treatment ( $p=0.56$, Mann-Whitney test).

F: Density of intermittent boutons for control and 2-AG treated axons. Bars represent mean \pm SEM. Dots represent individual axons. Intermittent bouton density is significantly increased after treatment $\left(^{*}, p<0.05\right.$, Mann Whitney test).

G: Cumulative probability distributions of the growth coefficient of persistent boutons that were exposed to control ACSF (black; $n=18$ I boutons; $N=9$ ) or $100 \mu M$ 2-AG (red; $n=135$ boutons; $N=8$ ).

$\mathbf{H}$ : Cumulative probability distributions of the growth coefficient of non-persistent boutons that were exposed to control ACSF (black; $n=162$ boutons; $N=9$ ) or $100 \mu M$ 2-AG (red; $n=167$ boutons; $N=8$ ). *, $p<0.05$ Mann-Whitney test.

I: Cumulative probability distributions of the growth coefficient of non-persistent boutons that were exposed to control ACSF (black; $n=195$ boutons; $N=5$ ) or $100 \mu M$ 2-AG (red; $n=204$ boutons; $N=6$ ) in the presence of $5 \mu M$ of the $\mathrm{CBI}$ receptor antagonist $\mathrm{AM} 25 \mathrm{I}$.

J: Cumulative probability distributions of the growth coefficient of non-persistent boutons that were exposed to control ACSF (black; $n=160$ boutons; $N=6$ ) or $200 \mathrm{ng} / \mathrm{ml} \mathrm{BDNF}$ (blue; $\mathrm{n}=195$ boutons; $\mathrm{N}=6$ ).

K: Growth coefficient of non-persistent boutons with low and high baseline intensity. Bars represent mean \pm SEM. ***, $\mathrm{p}<0.00 \mathrm{I}$, 2-way ANOVA followed by Sidak multiple comparisons test.

L: Normalized bouton intensity over time for non-persistent boutons with low baseline intensity, that were exposed to control ACSF (black, $n=54$ boutons) or 2-AG (red, $n=66$ boutons). *, $p<0.05,2$-way ANOVA followed by Sidak multiple comparisons test. Error bars represent SEM.

al., 20I4; Meyer et al., 20I4; Murthy et al., 200 I; Pierce and Lewin, 1994). As boutons might change in size after local 2-AG application (without changing bouton category), we quantified the integrated fluorescence intensity (as a proxy for bouton size, see Methods for details) at every bouton location at every time point.A bouton location is defined as a location where a bouton appears for at least two time points, but intensity was quantified at these locations in all time points (irrespective of whether the bouton was absent or present). Next, we defined a growth coefficient (see Methods for details), such that a positive growth coefficient reflects bouton growth while a negative growth coefficient reflects bouton shrinkage. We observed that persistent boutons typically have a small (positive or negative) growth coefficient. This reflects that persistent boutons are large and do not fluctuate a lot in size. Local application of 2-AG does not induce any change in the growth coefficients of persistent boutons (Figure IG, $\mathrm{p}=0.77, \mathrm{MW}$ ). Non-persistent boutons show a wider distribution of growth coefficients, reflecting their dynamic nature. Application of 2-AG leads to a remarkable right-shift of the distribution of growth coefficients, suggesting that non-persistent boutons that were exposed to 2-AG are more prone to grow (Figure IH, $\mathrm{p}=0.02, \mathrm{MW}$ ). This effect is completely blocked when axons are exposed to 2-AG in the presence of $\mathrm{CBI}$ receptor-antagonist AM25 I (Figure II, $\mathrm{p}=0.69, \mathrm{MW}$ ). Locally applying BDNF, another retrograde messenger, does 
not lead to more bouton growth, showing that the effect is specific to 2-AG (Figure IJ, $\mathrm{p}=0.09, \mathrm{MW})$.

Because the non-persistent category of boutons contains boutons with a wide range of dynamics, it is not straightforward to interpret what exactly is causing the increase of the growth coefficient. We therefore compared the average growth coefficient of non-persistent boutons with a low or a high average baseline intensity. Low baseline intensity boutons roughly represent the boutons that are absent, very rarely present, or very small during the baseline period, while high baseline intensity boutons are boutons that are often present or large during the baseline period. We observe that low baseline intensity boutons are completely responsible for the increase in growth coefficient, while high baseline intensity boutons do not respond at all (Figure IK, 2-way ANOVA, $\mathrm{p}<0.01$ for treatment, baseline
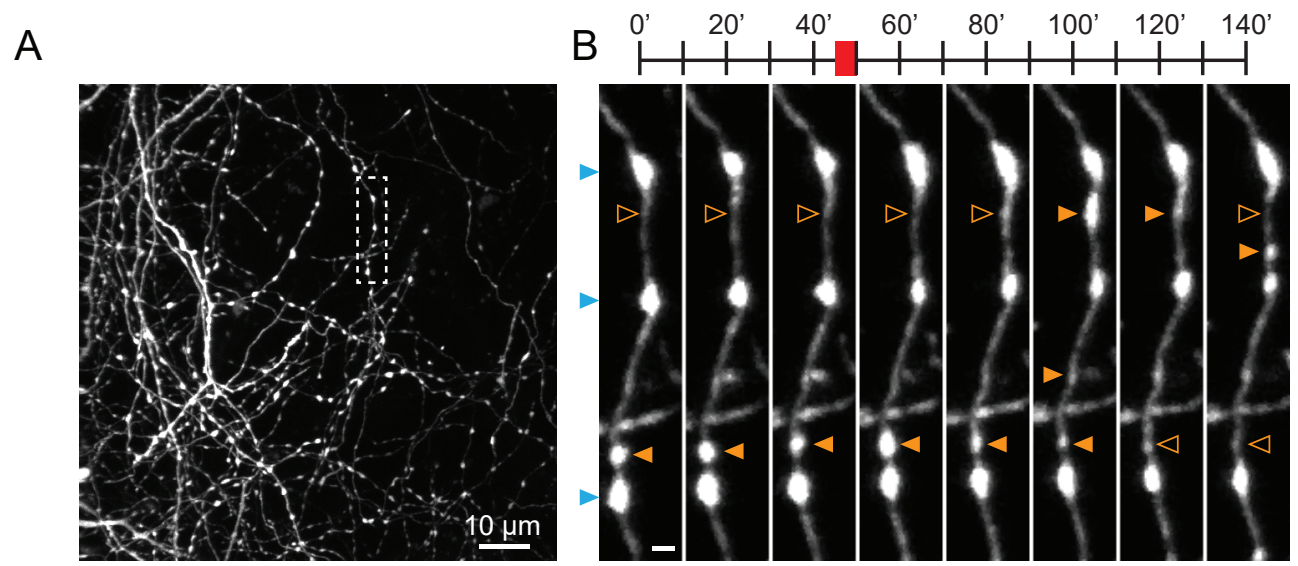

C

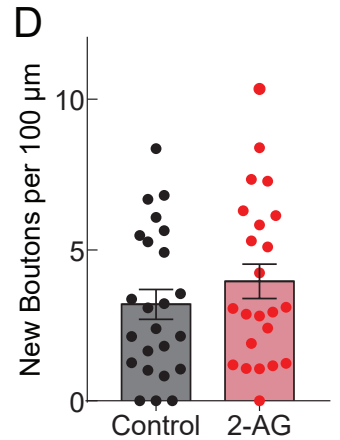

Figure 2. Bath application of 2-AG does not robustly affect bouton dynamics.

A: Example of a typical imaging area used for the analysis of bouton dynamics. Maximum projection, median filtered for illustration purposes. The dashed box indicates the area that is shown enlarged in (B).

B: Enlarged image of the area indicated by the dashed box in (A), over time. Images were taken every 10 minutes, every other image is shown. Between the fifth and sixth timepoint, DMSO or 2-AG treatment is applied for 5 minutes. Blue arrowheads indicate persistent boutons while orange arrowheads indicate an absent (open) or present (filled) non-persistent bouton. Scale bar, I $\mu \mathrm{m}$.

C: Bouton density of the different non-persistent bouton categories for control (black; $\mathrm{n}=24$ axons, $\mathrm{N}=6$ slices) and 2-AG (red; $\mathrm{n}=23$ axons, $\mathrm{N}=6$ slices) treated axons. Bar represents mean \pm SEM. 2-AG bath application does not induce robust changes in bouton dynamics.

D: Density of new boutons for control and 2-AG treated axons. Bar represents mean \pm SEM. Dots represent individual axons. There is a trend towards a higher new bouton density after 2-AG bath application ( $P=0.35$, MannWhitney test). 
intensity, and interaction; followed by Sidak MC, $p<0.00$ I for low baseline intensity, $p=0.89$ for high baseline intensity). When we look at the average intensity of boutons with low baseline intensity over time, we observe that $\sim 20$ minutes after local application, bouton intensity is significantly increased compared to control (Figure IL). This is a similar time scale as was observed for bouton growth after excitatory synapse stimulation, as described in chapter 3 (See Figure IN,P of chapter 3). Together, these results strongly suggest that brief and local application of the endocannabinoid 2-AG is sufficient to promote inhibitory bouton growth, which is in agreement with our results in chapter 3.

Next, we asked if longer and global application of 2-AG would lead to a more robust effect on bouton dynamics. We performed two-photon imaging of large stretches of GFP-labeled inhibitory axons in organotypic slices of GAD65-GFP mice (Figure 2A,B). After acquiring 5 baseline time points (imaging every 10 minutes), we performed bath application of $100 \mu \mathrm{M}$ 2-AG or control solution in ACSF for 5 minutes, after which the treatment was washed out with regular ACSF. We then continued to acquire images for 10 more time points. For 2-4 axons per slice, we quantified bouton dynamics by classifying boutons in the different categories. In general, bouton densities in the different categories correspond well with bouton densities as measured in the local application experiments. Once again, we observe a small trend towards more new boutons after 2-AG application (Figure IC,D, $p=0.35, M W$ ). These data suggest that bath application of 2-AG for 5 minutes has a similar effect as local application for I minute. We therefore conclude that while we managed to reproduce a similar trend on new boutons, longer and more global application of 2-AG does not lead to a more robust increase in the density of new boutons.

\section{Repeated brief CBI receptor activation leads to an increase in mIPSC frequency after $24 \mathrm{~h}$}

Inhibitory boutons are considered to be a morphological correlate of inhibitory synapses: $\sim 80 \%$ of inhibitory boutons colocalizes with inhibitory pre- and postsynaptic markers (Frias et al., 2019; Wierenga et al., 2008). For non-persistent boutons, the level of colocalization with synaptic markers is lower and is dependent on how long the bouton is present (Frias et al., 2019; Schuemann et al., 20I3). Even on the minute-to-hour time scale, a substantial part of the newly formed boutons are likely to be functional synapses (see Figure SIG-I in chapter 3). The gradual increase in colocalization with synaptic markers suggests that newly formed boutons slowly mature to functional synapses. We therefore set out to study if the bias towards bouton growth that is triggered by endocannabinoid signaling on short time scales can lead to the formation of functional inhibitory synapses on longer time scales.

To study the long-term effect of endocannabinoid signaling in inhibitory synapse formation, we treated organotypic hippocampal slices by placing the insert with the slice in culturing medium containing $100 \mu \mathrm{M}$ 2-AG or DMSO vehicle for 20 minutes (Figure 3A). To ensure that the treatment penetrated well into the slice, a drop of culturing medium containing the treatment was placed on top of the slice. The treatment period of 20 minutes was chosen to briefly activate $\mathrm{CBI}$ receptors, while ensuring sufficient penetration into the slice. Afterwards, the insert with the slice was placed in regular culturing medium and a drop of culturing medium was placed on top of the slice to wash out the treatment. This procedure was repeated three times in total ( $2 \mathrm{~h}$ intervals) to increase the likelihood of inducing a robust effect. $24 \mathrm{~h}$ later, we performed patch clamp electrophysiology on CAI pyramidal neurons and measured miniature inhibitory postsynaptic currents (mIPSC). 
A
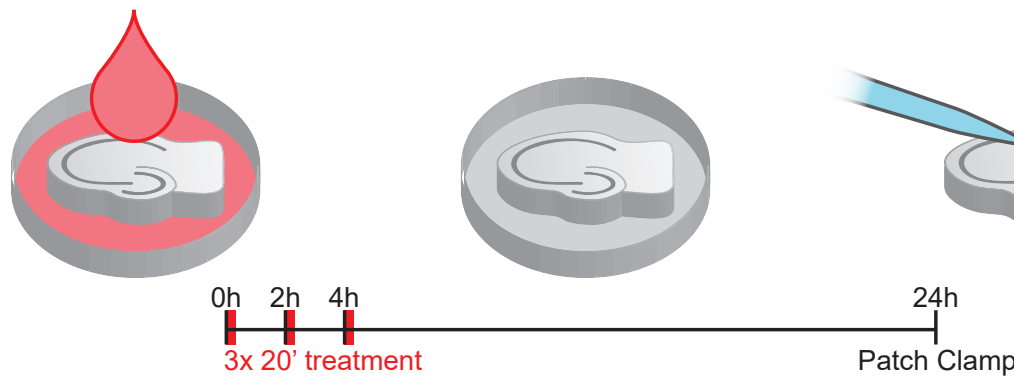

B

DMSO
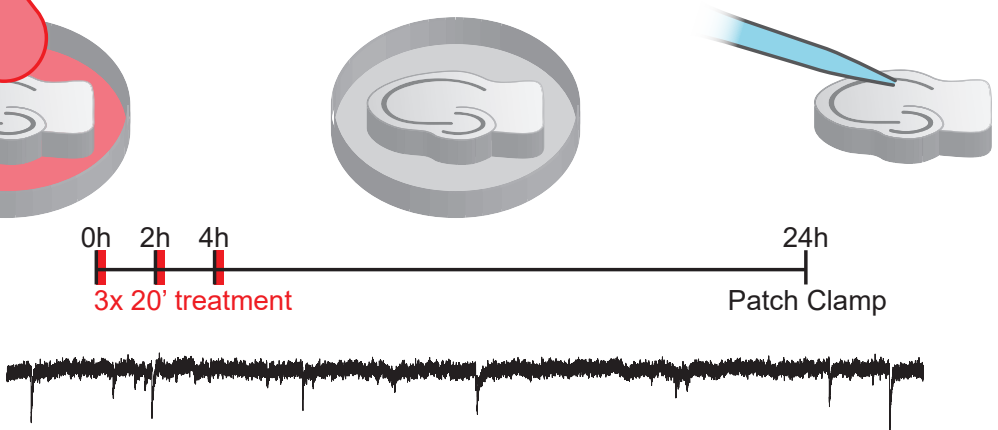

$4 \mathrm{~h}$

Patch Clamp

\section{2-AG}
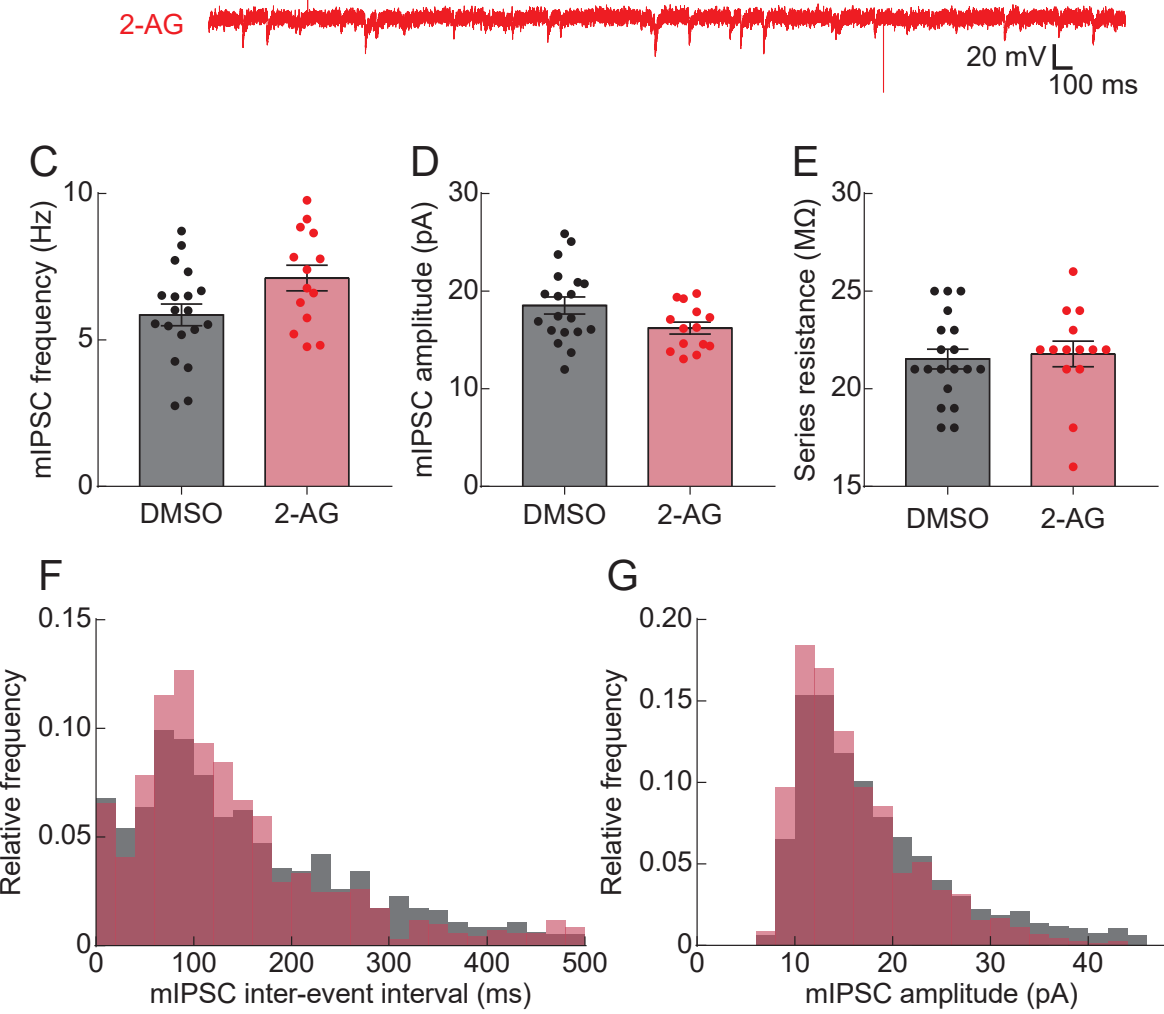

G

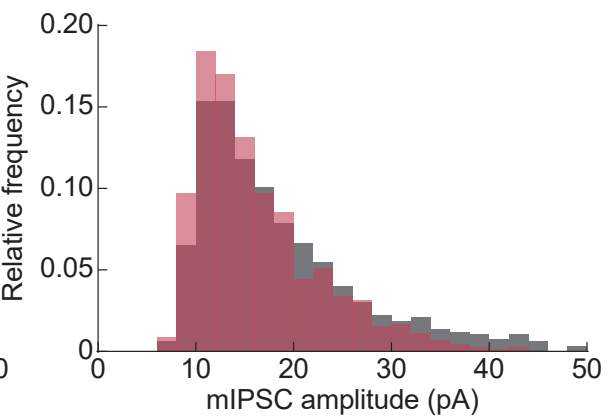

Figure 3. Repeated brief 2-AG treatments lead to an increased mIPSC frequency after 24h.

A: Slices were placed with the culturing insert in medium containing $100 \mu \mathrm{M} 2$-AG or DMSO control and a drop of treatment medium was placed on top of the slice to ensure penetration. After 20 minutes, slices were placed back in fresh culturing medium. This treatment was performed three times in total, spaced at $2 \mathrm{~h}$ intervals. After the last treatment, slices were kept in fresh culturing medium until they were used for patch clamp experiments $24 \mathrm{~h}$ later. B: Example electrophysiological traces showing mIPSCs recorded in slices that were treated with DMSO (top; black) or 2-AG (bottom; red).

C: $\mathrm{mIPSC}$ frequency of CAI pyramidal neurons treated with DMSO (black; $\mathrm{n}=19$ cells, $\mathrm{N}=5$ slices) or 2-AG (red; $n=14$ cells, $N=5$ slices). Bars represent mean \pm SEM. Dots represent individual cells. $p=0.0504$, Mann-Whitney test. D: mIPSC amplitude of CAI pyramidal neurons treated with DMSO (black) or 2-AG (red). Bars represent mean \pm SEM. Dots represent individual cells. $p=0.08$, Mann-Whitney test.

E: Series resistance of CAI pyramidal neurons treated with DMSO (black) or 2-AG (red). Bars represent mean \pm SEM. Dots represent individual cells. $p=0.47$, Mann-Whitney test. 
F: Relative frequency distribution of mIPSC inter-event-interval (IEI) of cells treated with DMSO (gray; $\mathrm{n}=927$ mIPSCs, $\mathrm{N}=19$ cells) or $2-A G$ (pink; $n=686$ mIPSCs, $N=14$ cells).

G: Relative frequency distribution of mIPSC amplitude of cells treated with DMSO (gray; $n=950$ mIPSCs, N=19 cells) or 2 -AG (pink; $n=700$ mIPSCs, $N=14$ cells).

A mIPSC reflects the spontaneous fusion of a synaptic vesicle and the subsequent activation

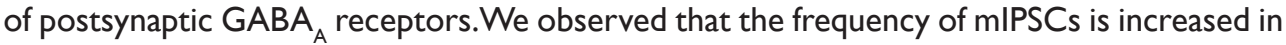
the cells that were treated with 2-AG (Figure 3B,C). This trend towards a higher frequency is nearly significant $(p=0.05, M W)$. When looking at the histogram of the inter-event interval (IEI), we observe that there is a relative increase of short $(50-170 \mathrm{~ms})$ IEls, causing an increase in average frequency (Figure 3F). An increase of mIPSC frequency indicates an increased spontaneous vesicle fusion, and therefore could reflect an increase in the total number of inhibitory synapses or an increase in release probability.

The average mIPSC amplitude shows a trend towards decrease in 2-AG treated cells (Figure 3B,D, $p=0.08, M W$ ). This decrease is not caused by a difference in series resistance (Figure $3 E, p=0.47, M W$ ). A decreased average mIPSC amplitude suggests that on average, inhibitory postsynapses are weaker.The histogram of mIPSC amplitude shows that there is a relative increase of 9-15 pA mIPSCs (Figure 3G). Together, these data show that after 2-AG treatment we observe more mIPSCs, and mIPSCs are typically of lower amplitude. This is in agreement with the 2-AG treatment causing an increase in the number of functional inhibitory synapses. It is likely that after 24 hours, new synapses are still in the process of maturation. The amplitude associated with new synapses is therefore low and reduces the average amplitude. On longer time scales, new synapses will likely become stronger. Together, these results are in agreement with the hypothesis that brief stimulation of the endocannabinoid system promotes bouton growth on shorter time scales, leading to an increase of functional inhibitory synapses on longer time scales.

\section{Prolonged activation of $C B \mid$ receptors leads to a decrease in the number of boutons and synapses}

Next, we tested the effect of prolonged stimulation of the eCB system on inhibitory synapses. Prolonged stimulation could lead to a more robust increase in inhibitory synapses. On the other hand, It has been reported that the $\mathrm{CBI}$ receptor can desensitize and internalize upon prolonged activation by agonists (Coutts et al., 200I; Hsieh et al., 1999; jin et al., 1999; Thibault et al., 2013; Wu et al., 2008). We placed organotypic hippocampal slices of the GAD65-GFP mouse line in culturing medium containing $100 \mu \mathrm{M}$ 2-AG or DMSO vehicle for 48 hours (continuous treatment, Figure 4A). In parallel, we treated slices with $5 \mu M$ of the $\mathrm{CBI}$ receptor antagonist $\mathrm{AM} 25 \mathrm{I}$ to study the effect of basal endocannabinoid release in the slice. We regularly placed a drop of culturing medium on top of the slice to ensure sufficient penetration of the treatment. After $\mathbf{4 8}$ hours, we fixed the slices and performed immunohistochemistry and confocal imaging.

First, we tested whether inhibitory bouton density was affected. We quantified bouton density based on the GFP fluorescence for up to eight axons per image stack and acquired up to five image stacks per slice (Figure 4B). Prolonged manipulation of the endocannabinoid system affects bouton density (Figure 4C,F, p<0.0I, ANOVA; ns, Sidak MC). We observe a trend towards lower bouton density after 2-AG treatment. Preventing CBI receptor activation altogether leads to the opposite effect: treatment with AM25I leads to an increase in bouton density. These data are in contrast with our results obtained with shorter 
A

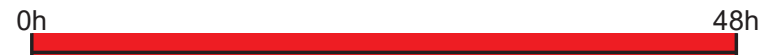

B

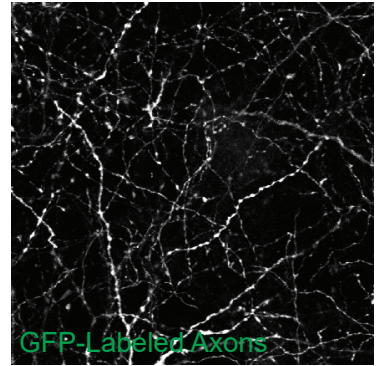

48h treatment

Fixation \& IHC

$\mathrm{C}$
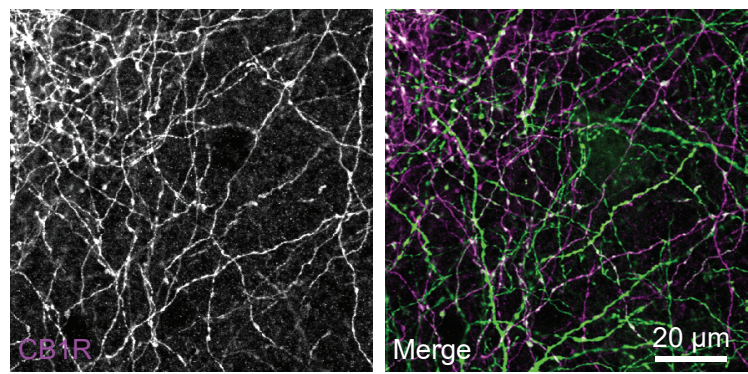

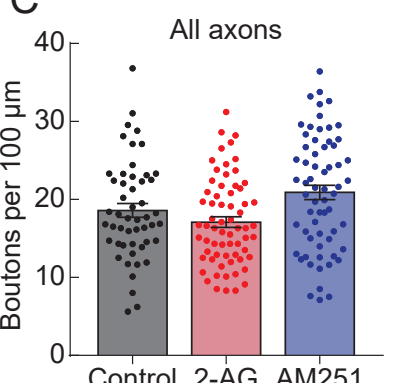

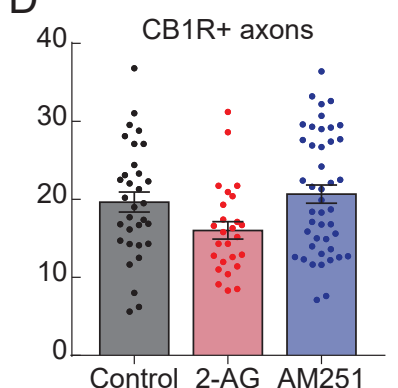

$\mathrm{E}$
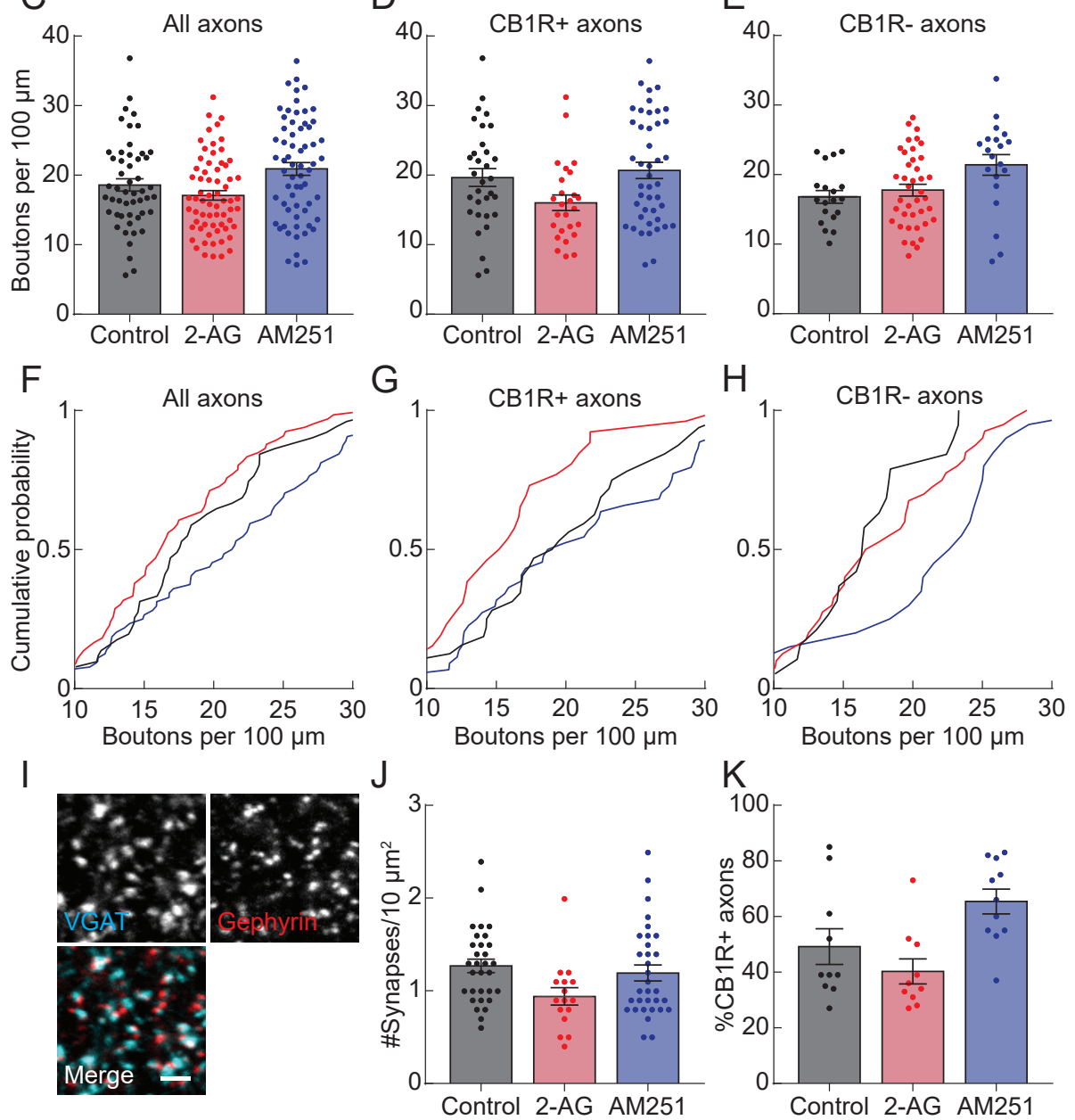

Figure 4. Prolonged stimulation of the eCB system reduces inhibitory synapse density and $C B I$ receptor levels. A: Slices were treated continuously for 48 hours with $100 \mu$ 2-AG, $5 \mu$ M AM25I or DMSO control, before fixation and immunohistochemistry.

B: Example of confocal images (maximum projection) of treated slices. GFP-labeled inhibitory axons (green; left), staining for the $\mathrm{CBI}$ receptor (magenta; middle), and merge (right). 
C-E: Bouton density for all axons $(C)$ in slices that were treated with DMSO control (black; $n=5$ I axons, $N=9$ image areas), 2-AG (red; $n=66$ axons, $N=10$ image areas), or $A M 25$ I (blue; $n=64$ axons, $N=I$ I image areas). Axons were categorized as $C B \mid R+$ axons ((D), n=32, 26 and 44, respectively) and $C B I R$ - axons $((E), n=\mid 9,40$ and 20, respectively). Bars represent mean \pm SEM. Dots represent individual axons. One-way ANOVA is significant for all graphs (all: $p<0.01 ; C B I R+$ and $C B I R-, p<0.05$; Post-hoc Dunnett's multiple comparisons test is only significant for CBIR-:AM25I vs Control, $p<0.05$ )

F-H: Cumulative probability distributions of bouton density for all axons $(F), C B I R+(G)$ and $C B I R-(H)$ axons in slices that were treated with DMSO control (black), 2-AG (red), or AM25I (blue).

I: Example of confocal images (maximum projection) of treated slices. Staining for vesicular GABA transporter (VGAT, blue), gephyrin (red) and merge. Scale bar, $2 \mu \mathrm{m}$.

J: Number of synapses (colocalizations of VGAT and gephyrin puncta) per $10 \mu \mathrm{m}^{2}$ for slices that were treated with DMSO control (black; $n=31, N=9$ image areas), 2-AG (red; $n=16, N=4$ image areas), or AM25I (blue; $n=32, N=9$ image areas). Bars represent mean \pm SEM. Dots represent individual $10 \times 10 \mu \mathrm{m}$ boxes. One-way ANOVA: $\mathrm{p}=0.05 \mathrm{I}$; post-hoc Dunnett's multiple comparisons test: Control vs 2-AG, $p<0.05$.

K: Percentage of $C B I R+$ axons in slices that were treated with DMSO control (black; $n=10$ image areas, $N=3$ slices), 2-AG (red; $n=10$ image areas, $N=3$ slices), or $A M 25 I$ (blue; $n=10$ image areas, $N=3$ slices). Bars represent mean \pm SEM. Dots represent individual image areas. One-way ANOVA: $p<0.0$ I; post-hoc Dunnett's multiple comparisons test: ns).

treatments, suggesting that prolonged treatment induces different signaling pathways than short treatments.

As reported in chapter 3 (Figure S4 of chapter 3), roughly half of the GFP-positive inhibitory neurons in our model system shows clear $\mathrm{CBI}$ receptor expression. We therefore performed immunohistochemistry for the $\mathrm{CBI}$ receptor (Figure 4B). We divided the axons into axons that were positive for the $C B I$ receptor $(C B I R+)$ and axons that did not show clear $C B I$ receptor expression (CBIR-) and compared bouton densities (Figure 4D,E,G,H). $C B I R+$ and $C B I R$ - axons were both affected by the treatments $(C B I R+: p=0.03$, ANOVA; ns, Sidak MC. CBIR-: $p=0.02$, ANOVA; $p=0.02$ (AM25I vs Control), Sidak MC). The decrease in bouton density after 2-AG treatment is due to a specific decrease of boutons in CBIR+ axons, while CBIR- axons are unaffected (Figure 4G). This suggests that 2-AG directly and selectively affects $C B I R+$ axons through axonal $C B I$ receptors. On the other hand, the increase in bouton density after AM25I treatment seems to occur mostly in CBIR-neurons (Figure $4 \mathrm{H}$ ). This surprising observation suggests that preventing $\mathrm{CBI}$ receptor activation has a profound effect on axons that express low levels of the $C B I$ receptor, that are not detectable with immunohistochemistry. Alternatively, CBIR- axons are affected by blocking $\mathrm{CBI}$ receptor activation indirectly - for example, their bouton density could be dependent on signaling via $\mathrm{CBI}$ receptor-expressing astrocytes (Navarrete et al., 20I4; Navarrete and Araque, 2008).

In the same slices, we also performed immunohistochemistry for the presynaptic inhibitory synapse markerVGAT (vesicular GABA transporter) and the postsynaptic inhibitory synapse marker gephyrin (Figure 4I). We selected four ROls of $10 \times 10 \mu \mathrm{m}$ per image stack and quantified the number of colocalizing puncta ofVGAT and gephyrin.As the presence ofVGAT and gephyrin indicates functional pre- and postsynaptic compartments, a colocalization can be considered a putative inhibitory synapse. We therefore quantified the synapse density by counting the number of colocalizations. Upon treatment with 2-AG, there is a trend towards a decrease in synapse density (Figure 4J, $p=0.05$, ANOVA). This decrease seems to be caused by a decrease in the number of gephyrin puncta $(p<0.0$ I, ANOVA; $<<0.0$ I, Sidak MC; data not shown) and a trend towards decrease in the number of VGAT puncta ( $p=0.06$, ANOVA; data not shown). This is in agreement with the effect we observed on bouton density. On the other hand, treatment with AM25I does not seem to affect the number of inhibitory synapses. 
A

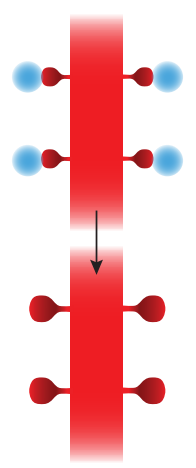

D $\quad$ AM251

CB1R Block

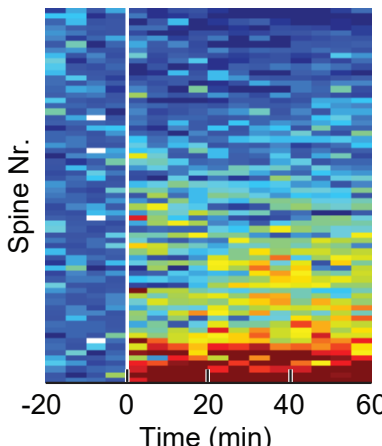

B

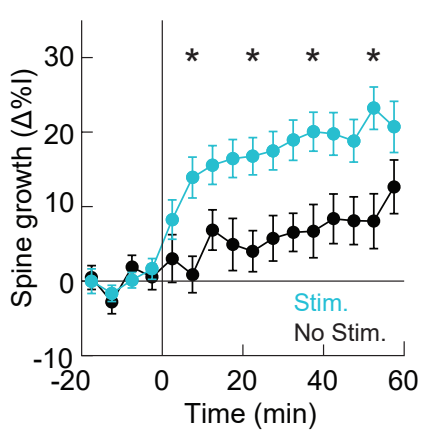

E

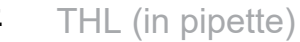

2-AG Production Block

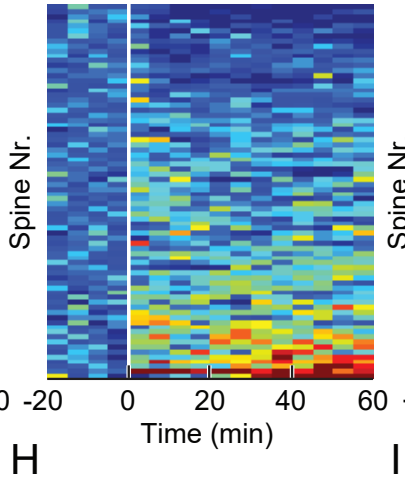

C

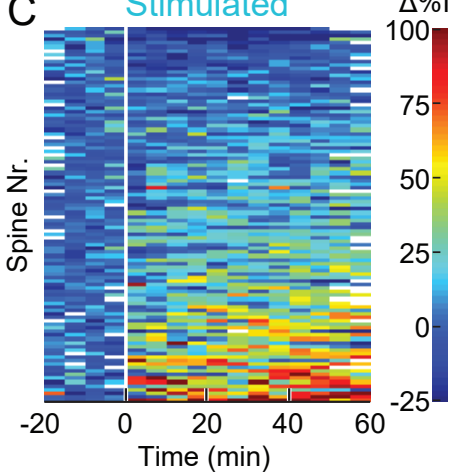

F

2-AG Degradation Block
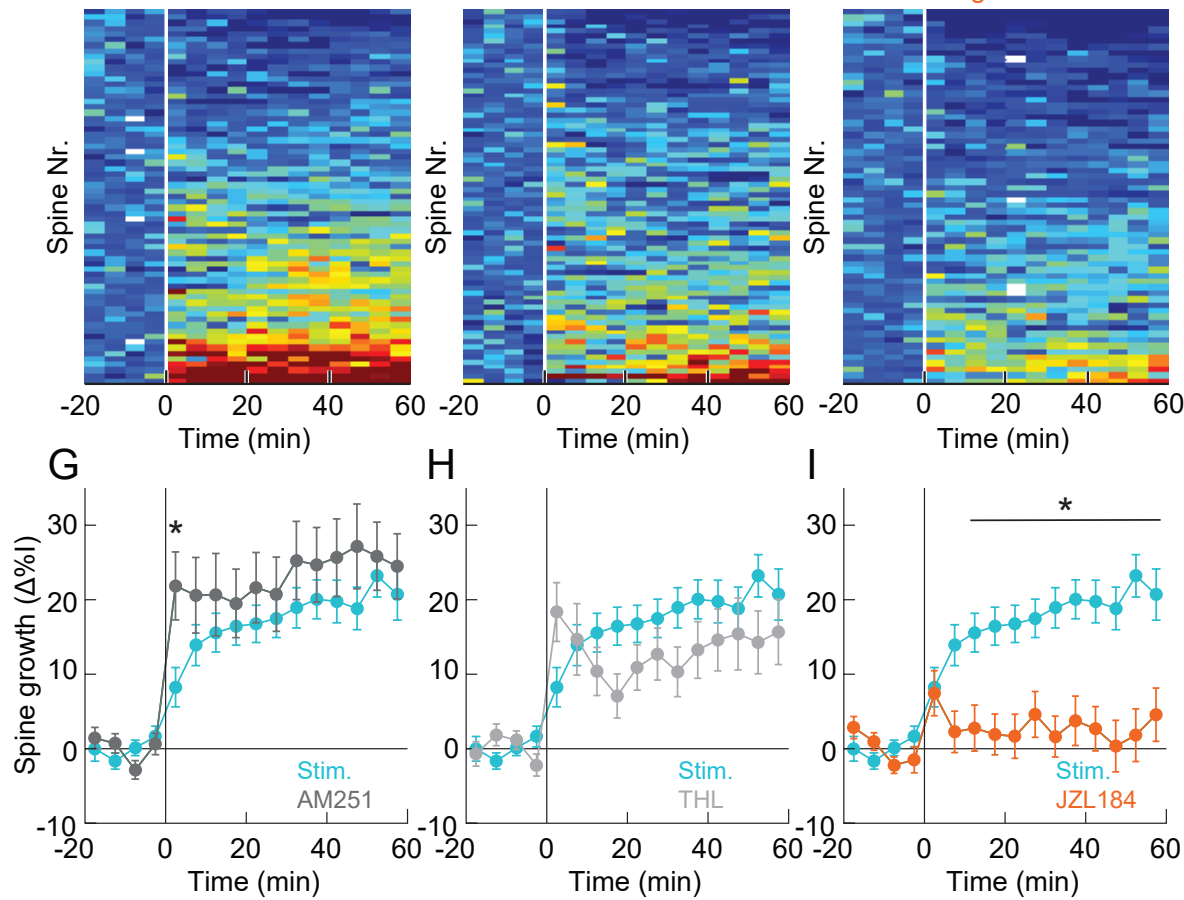

Figure 5. Manipulations of the endocannabinoid system affect structural LTP after glutamate uncaging.

A: A cluster of four small spines was stimulated near-simultaneously 30 times at $0.5 \mathrm{~Hz}$ using two-photon glutamate uncaging. After the stimulus, we continued to monitor the spines and quantify the growth of the spine head.

B: Spine growth, quantified as the increase in relative intensity $(\Delta \%)$ over time (as shown in chapter 3$)$. Local spine stimulation induced a gradual increase in spine size over time ('Stim', blue; $n=104$ spines, $N=32$ ), which was absent in the non-stimulated condition ('No Stim', black; $\mathrm{n}=6 \mathrm{I}$ spines, $\mathrm{N}=19$ ). ${ }^{*}, \mathrm{p}<0.05$, two-way ANOVA with post-hoc Sidak multiple comparisons test on all datasets (B, G, H and I), compared to Stim. Bars represent SEM.

C: Heat map showing spine volumes over time of all experiments where we performed spine stimulation ("Stimulated", $\mathrm{n}=104$ spines, $\mathrm{N}=32$ ). Each row represents a single experiment. The vertical white line indicates the end of the baseline period (first 20 minutes). White squares indicate missing time points. Experiments are sorted by maximal spine growth.

D-F: Heat maps showing spine volumes over time of all experiments where we performed spine stimulation in presence of $5 \mu \mathrm{MAM} 25 \mathrm{I}$ in the bath (D; "CBIR Block", $\mathrm{n}=67$ spines, $N=18$ ), $5 \mu \mathrm{M}$ THL in the patch pipette (E; "2-AG Production Block", $n=76$ spines, $N=21$ ) or 100 mm JZLI84 in the bath ( $F$; "2-AG degradation Block", $n=64$ spines, $\mathrm{N}=18$ ). Each row represents a single experiment. The vertical white line indicates the end of the baseline 
period (first 20 minutes). White squares indicate missing time points. Experiments are sorted by maximal spine growth.

G-I: Spine growth over time for stimulated spines in the presence of AM25I (G; dark gray), THL (H; light gray) or JZLI84 (l; orange). All graphs show spine growth as measured in absence of any drugs (blue, "Stim") as a reference. *,p<0.05, two-way ANOVA with post-hoc Sidak multiple comparisons test on all datasets, compared to Stim. Bars represent SEM.

Together, these data suggest that prolonged activation of the $\mathrm{CBI}$ receptor can decrease the number of boutons and synapses, while preventing $C B I$ receptor activation causes an increase. This is in contrast with the effect we observed with shorter treatments of 2-AG, which lead to more inhibitory boutons and synapses. In our experiments, we observed that drug treatment affects the fraction of $C B I R+$ axons (Figure 4K, $p<0.01$, ANOVA; ns, Sidak MC). Under control conditions the fraction of CBIR+ GFP-labeled axons was $49 \pm 6 \%$, but this fraction was decreased upon 2-AG treatment $(40 \pm 5 \%)$ and strongly increased after AM25I treatment $(65 \pm 4 \%)$. These results suggests that tonic endocannabinoid signaling affects receptor expression levels, causing a change in the percentage of axons that is detectable as CBIR+.

Our data shows that prolonged manipulations of the eCB system affects the level of $\mathrm{CBI}$ receptors, but also has an effect on inhibitory synapse formation that is not yet fully understood. The local coordination of inhibitory bouton formation observed in chapter 3 is likely mediated by an acute and local increase in $\mathrm{ECB}$ production. The results obtained in this experiment might therefore not be very representative of the role of eCB signaling in this local coordination.

\section{The endocannabinoid system can also affect excitatory synaptic plasticity}

While eCBs are most famous for their effects on inhibitory synapses, they can also affect excitatory synapses. Indirectly, the endocannabinoid system is thought to facilitate LTP at excitatory synapses by locally reducing inhibition, a form of metaplasticity (Carlson et al., 2002; Chevaleyre and Castillo, 2004; Xu et al., 20I4). Recent studies have shown that eCBs can also directly mediate presynaptically expressed LTP at corticostriatal synapses and perforant path-dentate granule cell synapses (Cui et al., 2015, 2016; Wang et al., 2016, 20I8). On the other hand, there are reports that observe a reduction of LTP with eCB signaling that might be mediated through postsynaptic $C B I$ receptors (Maroso et al., 2016; Monory et al., 20 I5; Stella et al., 1997) However, at the level of the single synapse, it has not yet been studied how eCB signaling influences long-term potentiation.

In chapter 3, we used two-photon glutamate uncaging on excitatory synapses to trigger the growth of an inhibitory presynaptic bouton. The glutamate uncaging protocol is designed to induce long-term potentiation, and after glutamate uncaging, we observed spine growth (Figure 5A-C) which serves as a morphological proxy for LTP (as described in chapter 2). We repeated our experiments in presence of drugs that interfere with $\mathrm{eCB}$ signaling, to show the involvement of the $\mathrm{eCB}$ system in inhibitory bouton formation (Figure 4 in chapter 3 ). These experiments however also allow us to assess the effect of manipulations of the eCB system on excitatory synaptic plasticity. Here, we reanalyze our previous data and quantify the growth of the spines that were stimulated in these experiments.

First, we performed two-photon glutamate uncaging on four excitatory spines in the presence of $5 \mu \mathrm{M}$ of the $\mathrm{CBI}$ receptor antagonist $\mathrm{AM} 25 \mathrm{I}$. Spine growth was intact when $\mathrm{CBI}$ receptor 
was blocked and was similar to the level of spine growth in absence of pharmacological manipulations, although the average spine growth seemed to accelerate (Figure 5D,G). Next, we stimulated spines while the postsynaptic neuron was perfused with $5 \mu M T H L$, an inhibitor of the 2-AG synthesis enzyme DAGL. Under these conditions, spine growth seems to be largely unaffected (Figure 5E,H). Finally, we stimulated spines in presence of $100 \mathrm{nM}$ JZI84, an inhibitor of the enzyme MAGL which is responsible for the degradation of 2-AG. When 2-AG degradation is inhibited and 2-AG levels are therefore likely increased, spine growth is completely abolished (Figure $5 \mathrm{~F}, \mathrm{I}$ ). It is important to note that in this condition, inhibitory bouton growth is intact (Figure 4C-E of chapter 3).

These results suggest that increasing 2-AG concentration by preventing degradation can abolish long-term plasticity of excitatory synapses. Our results best correspond with data presented by Maroso and colleagues. They show that in superficial CAI pyramidal cells, postsynaptically expressed $\mathrm{CBI}$ receptors can trigger a signaling cascade that upregulates the $\mathrm{HCN}$-mediated $\mathrm{I}_{\mathrm{h}}$-current and thereby strongly reduces LTP (Maroso et al., 20I6). To fully understand our observations regarding spine growth, we need to explore whether postsynaptically expressed $C B I$ receptor plays a role here as well, or whether spine growth is affected through an indirect mechanism. 


\section{Discussion \& Outlook}

In this chapter, we further explored the role of eCB signaling in inhibitory bouton and synapse formation. We showed that brief and local application of the eCB 2-AG to inhibitory axons leads to more bouton growth, similar to the results that were presented in chapter 3. Repeated treatments with 2-AG can lead to an increase of mIPSC frequency 24 hours later, in agreement with an increase in the number of inhibitory synapses. Prolonged manipulations of the eCB system lead to changes in $\mathrm{CBI}$ receptor expression and effects on inhibitory synapse formation that are opposite to what was observed after brief treatments. We also presented data that suggests that manipulations of the $\mathrm{CCB}$ system can affect the structural plasticity of single excitatory synapses. Together, our data show that the eCB system is highly versatile and plays important roles in many plasticity processes.

In chapter 3, we observed an increased likelihood of bouton growth on an axon-dendrite crossing after stimulation of excitatory synapses on the dendrite. Most of these boutons grew on an "empty" axonal stretch, while in a small number of experiments a small varicosity that was already present on the axon grew into a bouton after stimulation. We therefore hypothesized that mimicking the signaling events by locally applying 2-AG would lead to an increase in the number of new boutons. Our experiments demonstrated that both local (I $\mathrm{min}$ ) and global (5 $\mathrm{min}$ ) application of 2-AG leads to a small trend towards more new bouton formation. The relative weakness of this effect might be caused by the large stretches of axons that are exposed to 2-AG. eCB signaling is thought to spread about $10 \mu \mathrm{m}$ (Chevaleyre and Castillo, 2003), while in our experiments $\sim 30 \mu \mathrm{m}$ of the axon (local application) or even the entire axon (global application) is exposed. When signaling is triggered over a wider area, boutons-in-the-making might compete for the same resources (Frias and Wierenga, 2013; Staras, 2007; Staras et al., 2010;Wierenga, 2017), therefore reducing the effect on new bouton formation. With local application, we do observe a robust effect on the maximal bouton growth coefficient, a less strict measurement that is dependent on changes in bouton volume as well as the appearance of new boutons and can therefore pick up more subtle effects. This result demonstrates that axons that are exposed to 2-AG are more prone to form new boutons and/or grow from small varicosities into larger boutons.

We do not yet fully understand what it takes to make an inhibitory bouton, or how this could be regulated by eCB signaling. Typically, $\mathrm{CBI}$ receptors are coupled to a $\mathrm{G} \alpha_{\mathrm{i} / \mathrm{o}}$ subunit and activation of the receptor leads to reduction of CAMP levels and PKA activity (Heifets and Castillo, 2009). It is well possible that the effect of eCB signaling on bouton growth is mediated by the $\mathrm{Ga}_{\mathrm{i} / \mathrm{o}}$ pathway. It would therefore be interesting to study the role of cAMP/PKA signaling in inhibitory bouton growth. It is however important to note that atypical signaling through other Ga subunits can occur. An atypical CBI receptor signaling pathway has been reported in immature neurons, where the $C B I$ receptor is expressed in axonal growth cones. Growth cone avoidance or collapse is regulated through CBI receptors coupled to an atypical $\mathrm{G \alpha}_{12 / 13}$ subunit, and activation of the receptor leads to RhoA and ROCK activation and actin cytoskeleton remodeling in the axon (Berghuis et al., 2007; Njoo et al., 20I5; Roland et al., 2014). A similar pathway might play a role in the growth of presynaptic boutons. Mature axons and boutons contain complex actin structures (Ganguly et al., 2015; Papandréou and Leterrier, 2018), and previous work from our lab has shown that promoting actin depolymerization can enhance bouton stabilization (Frias et al., 2019). This is likely regulated through the reduction of ROCK activity, while $\mathrm{Ga}_{12 / 13}$-coupled $\mathrm{CBI}$ receptor activation leads to an increase of ROCK activity. It is however possible that 
during different stages bouton growth and formation, different actin remodeling strategies are applied. Perhaps early formation and growth of a bouton requires an increase of ROCK activity and actin polymerization, while stabilization of boutons requires a reduction in ROCK activity and actin depolymerization. The activation of RhoA and ROCK, as well as other Rho GTPases, play important roles in the actin remodeling required for spine growth during long-term potentiation (see also chapter 2) (Murakoshi et al., 20I I), which might be analogous to bouton growth. Future experiments will shed a light on the type of G-protein signaling that is involved as well as the role of actin remodeling in inhibitory bouton growth.

The growth of an inhibitory presynaptic bouton is no warranty for the formation of an inhibitory synapse. We therefore performed electrophysiology experiments 24 hours after three brief treatments with 2-AG to study if eCB signaling could trigger the formation of functional synapses on longer time scales. We observed an increase in mIPSC frequency, which is in agreement with an increase in the number of inhibitory synapses. Alternatively this result can be explained by an increase in vesicle release probability. To distinguish between these, paired pulse ratio experiments should be performed. Additionally, immunohistochemistry experiments can be performed $24 \mathrm{~h}$ after treatment to analyze bouton densities and VGAT/ gephyrin colocalizations, potentially confirming an increase in inhibitory synapse number.

Apart from the effects we observed on inhibitory neurons, our data also suggest that excitatory synaptic plasticity is affected by manipulations of the eCB system. Specifically, twophoton glutamate uncaging-induced spine growth was completely abolished in the presence of an inhibitor of the 2-AG degrading enzyme MAGL. These results suggest that (over) activation of the $\mathrm{eCB}$ system can interfere with spine growth. To test if strong $\mathrm{CBI}$ receptor activation indeed prevents spine growth, local application of a $C B I$ receptor agonist should be combined with two-photon glutamate uncaging. Additionally, inhibiting MAGL in presence of a $\mathrm{CBI}$ receptor antagonist should restore spine growth if the effect is indeed mediated by $\mathrm{CBI}$ receptors. It has been shown that theta burst stimulation-induced LTP is abolished in superficial CAI pyramidal neurons in the presence of the synthetic $C B I$ receptor agonist WIN. CBI receptor signaling was localized to the postsynaptic dendrite and required modulation of $\mathrm{HCN}$ channels (Maroso et al., 2016). Knock-out of the CBI receptor in glutamatergic and GABAergic neurons specifically could elucidate the locus of $\mathrm{CBI}$ receptor signaling that is important for spine growth.

In this chapter, we focused on the role the eCB 2-AG. While we do not exclude a role for $A E A$ in the regulation of inhibitory synapse formation, there are two reasons for focusing on 2-AG. (I) As mentioned in the introduction, 2-AG is the predominant eCB in the hippocampus in terms of concentration. (2) In the glutamate uncaging experiments of chapter 3, we observed that intracellular perfusion with THL, a drug that inhibits the 2-AG producing enzyme DAGL, could abolish the enhancement of inhibitory bouton growth. As DAGL cannot synthesize AEA, this result suggests that specifically 2-AG mediates dendritic coordination of excitatory and inhibitory synapses. It however needs to be noted that in most experiments in this chapter we perform exogenous application of 2-AG to activate the $\mathrm{CBI}$ receptor. Repeating experiments with exogenous application of AEA will provide further insights in its role in these processes.

The CBI receptor is highly expressed in a subset of GFP-labeled inhibitory axons in our organotypic hippocampal slices, as demonstrated by immunohistochemistry (Figure 4). It is therefore most likely that our results can be explained by activation of the $\mathrm{CBI}$ receptor in these axons. Experiments with the antagonist AM25I, that displays strong selectivity for 
the $\mathrm{CB}$ I receptor over the $\mathrm{CB} 2$ receptor, prevented bouton growth upon local application (Figure I) and in chapter 3. However, we cannot completely exclude a contribution of the CB2 receptor or other atypical eCB receptors. While the $C B 2$ receptor is mostly present on immune cells, some studies have detected the $C B 2$ receptor and $C B 2$ receptor-mediated effects in the brain (den Boon et al., 20I2; Li and Kim, 20I5; Stempel et al., 20I6).Additionally, it has been shown that eCBs can activate the vanilloid receptor TRPVI, which is expressed throughout the brain, and mediate postsynaptic LTD (Chávez et al., 20l0; Cristino et al., 2006; Tóth et al., 2005). Future studies might elucidate the involvement of these receptors in inhibitory synapse formation. It would also be interesting to further look into the role of astrocytes and neurons with low levels of $\mathrm{CBI}$ receptor expression in inhibitory synapse formation and excitatory synaptic plasticity.

Our experiments with prolonged (48h) manipulations of the eCB system showed that the duration of $C B I R$ experiments can crucially affect the downstream effect. A prolonged exposure to 2-AG leads to a reduction in inhibitory boutons and synapses, while prolonged exposure to AM25I leads to an increase in inhibitory boutons. This is in strong contrast with the effects observed with shorter treatments, where 2-AG exposure lead to more bouton growth and an increase of mIPSC frequency. We furthermore observed that prolonged manipulations can affect the fraction of axons that are detected as positive for the $\mathrm{CBI}$ receptor. These changes in receptor expression could be due to changing rates of internalization in the presence of agonist or antagonist. Like many G-protein coupled receptors, $\mathrm{CBI}$ receptors internalize and desensitize upon activation. This is thought to happen continuously due to tonic $\mathrm{CBIR}$ activation and might be a mechanism of regulating receptor levels. (Coutts et al., 200I; Hsieh et al., 1999; Jin et al., 1999; Thibault et al., 2013; $\mathrm{Wu}$ et al., 2008). Prolonged exposure of the CBI receptor to agonist leads to more internalization, while prolonged exposure to an antagonist reduces the basal level of internalization, and these changes could affect the fraction of axons we can detect as CBIR+ using immunohistochemistry. A reduction of the surface expression level of the receptor for 48 hours potentially interferes with regular eCB-mediated synapse formation. This could explain why we observe a reduction of inhibitory boutons and synapses instead of the increase that was observed in experiments where we used brief treatments.

It has been suggested that the duration of $\mathrm{CBI}$ receptor activation (seconds versus minutes) is a crucial differences between the induction of short-lasting DSI or long-lasting eCB-LTD (Castillo et al., 2012; Chevaleyre and Castillo, 2003). In striatum, it has been shown that the level and duration of eCB release can even determine the difference between LTP and LTD (Cui et al.,20I6).In a more extreme case, we showed here that prolonged (48h) manipulations of the eCB system can lead to effects that are very different from brief treatments. These observations suggest that in the realm of eCB signaling, timing is crucial. It would therefore be of high importance to perform experiments that increase our understanding of the temporal aspects of eCB signaling. To assess the relevance of the different eCB-mediated processes that have been reported, it is also important to study under what circumstances and with what temporal pattern eCBs are released under physiological conditions.

Altogether, our data provide strong indications that endocannabinoid signaling is involved in the growth and formation of inhibitory presynaptic boutons, and on longer time scales, the formation of synapses. Furthermore, our data suggest that excitatory synaptic plasticity might be affected by eCB signaling as well. These results suggest a role for eCBs as local coordinators of excitation and inhibition. Their rapid synthesis upon glutamate receptor activation, local action radius, and large repertoire of effects on excitatory and inhibitory 
pre- and postsynapses makes them an ideal candidate to locally maintain coordination. However, eCB signaling is complex, many atypical signaling pathways have been reported, and concentration, duration and frequency of $\mathrm{eCB}$ release seems to matter significantly. Further research is therefore needed to elucidate the exact role of the eCB system in all these processes. 


\section{Methods}

\section{Experimental model and subject details}

\section{Mice}

In this study, male and female GAD65-GFP mice (López-Bendito et al., 2004) were used (bred as a heterozygous line in a C57BL/6JRj background). All animal experiments were performed in compliance with the guidelines for the welfare of experimental animals issued by the Federal Government of The Netherlands. All animal experiments were approved by the Animal Ethical Review Committee (DEC) of Utrecht University.

\section{Organotypic hippocampal slice cultures}

Organotypic hippocampal slice cultures were prepared at postnatal day 6-8 with a method slightly modified from Stoppini et al. (Stoppini et al., 1991), as described in chapter 3. In brief: after decapitation and hippocampal dissection, slices were chopped with a thickness of 400 $\mu \mathrm{m}$. Sliced were placed on culture inserts in culturing medium (consisting of $48 \% \mathrm{MEM}$, $25 \%$ HBSS, $25 \%$ horse serum, $30 \mathrm{mM}$ glucose and $12.5 \mathrm{mM}$ HEPES, $\mathrm{pH}$ set at 7.3-7.4 and osmolarity set at $325 \mathrm{mOsm}$ ) and stored in an incubator $\left(35^{\circ} \mathrm{C}, 5 \% \mathrm{CO}_{2}\right)$. Slices were used for experiments after at least 7 days in vitro.

\section{Method details}

\section{Two-photon microscopy}

Two-photon microscopy was performed as described in chapter 3. In brief: slices were transferred to the microscope recording chamber and continuously perfused with carbogenated $\left(95 \% \mathrm{O}_{2}, 5 \% \mathrm{CO}_{2}\right.$ ) artificial cerebrospinal fluid fluid (ACSF, consisting of (mM): I $26 \mathrm{NaCl}, 3 \mathrm{KCl}, 2.5 \mathrm{CaCl}_{2}$, I.3 $\mathrm{MgCl}_{2}, 26 \mathrm{NaHCO}_{3}, \mathrm{I} .25 \mathrm{Na}_{2} \mathrm{H}_{2} \mathrm{PO}_{4}, 20$ glucose and I Trolox). All images were taken using a $60 x$ water immersion objective on a customized two-photon microscope using a tunable femtosecond pulsed laser. For local application experiments (Figure I), image stacks ( $468 \times 468$ pixels, $5 \mathrm{I} .5 \times 5 \mathrm{I} .5 \mu \mathrm{m}$ with $0.5 \mu \mathrm{m}$ z-steps) were acquired at a wavelength of $910 \mathrm{~nm}$ every 5 minutes for 5 time points before and up to an hour after local application. During local application, the laser was tuned to $840 \mathrm{~nm}$ to visualize GFP and Alexa568 simultaneously, allowing us to estimate the puff area. For bath application experiments (Figure 2), image stacks ( $1024 \times 1024$ pixels, 93.5x93.5 $\mu \mathrm{m}$ with $0.5 \mu \mathrm{m}$ z-steps) were acquired at a wavelength of $910 \mathrm{~nm}$ every 10 minutes for 5 time points before and 10 time points after bath application. For spine growth experiments (Figure 5), image stacks ( $256 \times 256$ pixels, $28.4 \times 28.4 \mu \mathrm{m}$ with $0.5 \mu \mathrm{m}$ z-steps) were acquired at a wavelength of 840 or $870 \mathrm{~nm}$ to visualize GFP and Alexa568 simultaneously. Images stacks were acquired every 5 minutes for 4 time points before and up to an hour after spine stimulation.

\section{Local application of 2-AG or BDNF}

Slices were placed in a chamber that was continuously perfused with ACSF at room temperature. For the local application of 2-AG, thick-walled application pipettes were filled with HEPES-ACSF (consisting of (mM): I $35 \mathrm{NaCl}, 3 \mathrm{KCl}, 2.5 \mathrm{CaCl}_{2}, 1.3 \mathrm{MgCl}_{2}, 1.25$ $\mathrm{Na}_{2} \mathrm{H}_{2} \mathrm{PO}_{4}, 20$ Glucose, and 10 HEPES) + $50 \mu \mathrm{M}$ Alexa568 (for visualization) and $100 \mu \mathrm{M}$ 2-arachidonoylglycerol (2-AG, Tocris) + $0.1 \%$ DMSO or $0.1 \%$ DMSO control solution. In 
experiments when $\mathrm{CBI}$ receptors were blocked, $5 \mu \mathrm{M}$ AM25I was added to the bath and the local application solution. We targeted axons in stratum radiatum, but we cannot exclude that a small population of GFP-labeled somatically targeting boutons were exposed and analyzed. Before the start of the experiment, the application pipette was placed inside the top layer of the slice, close to an area with multiple GFP-expressing inhibitory axons under visual control (excitation wavelength $840 \mathrm{~nm}$ for simultaneous visualization of Alexa568 and GFP). We adjusted the pressure of application pulses to set the diameter of the application area to $\sim 30 \mu \mathrm{m}$ using a Picospritzer II $(4-10 \mathrm{mmHg})$. 2-AG was applied in 30 bursts at 0.5 $\mathrm{Hz}$ (3 pulses of $50 \mathrm{~ms}$ per burst) to mimic the spine stimulation protocol of chapter 3 . For BDNF application, we used $200 \mathrm{ng} / \mathrm{ml}$ Human recombinant BDNF (Merck) + 0. I\% bovine serum albumin (BSA, Sigma) or 0.1\% BSA control and 25-75 ms pulses at $2 \mathrm{~Hz}$ for 2 minutes.

\section{Bath application of 2-AG}

Slices were placed in a chamber that was continuously perfused withACSF that was maintained at $32{ }^{\circ} \mathrm{C}$ using a temperature controller (Luigs \& Neumann). $100 \mu \mathrm{M}$ 2-arachidonoylglycerol (2-AG,Tocris) + $0.1 \%$ DMSO or $0.1 \%$ DMSO control in ACSF was perfused over the slice for 5 minutes just before the acquisition of the $6^{\text {th }}$ time point. At the start of the $6^{\text {th }}$ time point, perfusion was switched back to regular ACSF.

\section{Spine stimulation}

The data on spine stimulation (Figure 5) that are presented in this chapter are reanalyzed from the experiments presented in Figure 4 of chapter 3 . In brief: cells were patched (internal solution consisting of (mM): I 40 K-gluconate, $4 \mathrm{KCl}, 0.5$ EGTA, 10 HEPES, 4 MgATP, 0.4 NaGTP, $4 \mathrm{Na}_{2}$ Phosphocreatine, and 30 uM Alexa568) to label the dendritic tree. After acquiring four baseline time points, an enlarged patch pipette was used for local application of $5 \mu \mathrm{M}$ DNI-glutamate dissolved in HEPES-ACSF.We stimulated four small spines 30 times at $0.5 \mathrm{~Hz}$ near-simultaneously $(0.1 \mathrm{~ms}$ interval) using I ms pulses of $730 \mathrm{~nm}$ light using a second femtosecond-pulsed laser.We then continued to image for up to an hour.

To block CBI receptors, $5 \mu$ MAM25 I (Tocris) was added to the bath and allowed to incubate for at least 40 minutes before the start of the experiment (so at least 60 minutes before spine stimulation). We also added $10 \mu \mathrm{M}$ AM25I in the application pipette. To block 2-AG production by the enzyme DAGL in the postsynaptic dendrite, $5 \mu \mathrm{M}$ of Tetrahydrolipstatin (THL, Orlistat, Sigma) was added to the patch clamp intracellular solution. The time between break-in and spine stimulation was typically 30-40 minutes. To block 2-AG degradation, 100 nM JZLI 84 (Tocris) was added to the bath and allowed to incubate for at least 40 minutes before the start of the experiment.

\section{Pharmacological treatments}

For electrophysiology experiments (Figure 3), slices were treated for 20 minutes with 100 $\mu \mathrm{M} 2-\mathrm{AG}+0.1 \% \mathrm{DMSO}$ or $0.1 \% \mathrm{DMSO}$ alone dissolved in culturing medium. Slices were placed with the cell culture insert in I $\mathrm{ml}$ of treatment medium and a $\sim 15 \mu \mathrm{l}$ drop was placed on top of the slice to ensure sufficient penetration of the treatment. 20 minutes later, the insert was moved to a new well with fresh culture medium, and again a drop was placed on top of the slice to wash out the drug. This procedure was repeated in total 3 times at 2 hour intervals. After the last treatment, the insert was passed through two wells of fresh culture medium before being placed in the final well with fresh medium, where it stayed overnight in 
absence of any treatment. During the entire treatment, slices were kept in an incubator ( 35 ${ }^{\circ} \mathrm{C}, 5 \% \mathrm{CO}_{2}$ ). 24 hours after the start of the first treatment, electrophysiology experiments were performed.

For immunohistochemistry experiments (Figure 4), slices were treated for 48 hours with

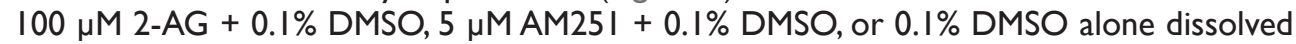
in culturing medium. Slices were placed with the cell culture insert in I $\mathrm{ml}$ of treatment medium and a $\sim 40 \mu \mathrm{l}$ drop was placed on top of the slice to ensure sufficient penetration of the treatment. A fresh drop was placed on the slice at the start and end of every day. After $24 \mathrm{~h}$, the culturing medium in the well was replaced with fresh culturing medium with fresh drugs. During the entire treatment, slices were kept in an incubator $\left(35{ }^{\circ} \mathrm{C}, 5 \% \mathrm{CO}_{2}\right)$ and were shielded from light to prevent light-induced degradation of the drugs. After 48 hours, slices were fixed and prepared for immunohistochemistry.

\section{Recording miniature inhibitory postsynaptic currents}

Slices were placed in a chamber that was continuously perfused with ACSF at $32-34{ }^{\circ} \mathrm{C}$. The $4 x$ air objective was used to locate the CAI region of the slice culture and to roughly position the patch pipette. Under a 60x water immersion objective (Olympus LUMFL N; I.I NA), whole-cell patch clamp of a CAI pyramidal cell neuron was performed. The patch pipette (3-7 $\mathrm{M} \Omega$ ) was filled with an internal solution with high chloride concentration (consisting of (mM): $70 \mathrm{~K}$-gluconate, $70 \mathrm{KCl}, 0.5$ EGTA, I0 HEPES, 4 MgATP, $0.4 \mathrm{NaGTP}, 4$ $\mathrm{Na}_{2}$ Phosphocreatine, and osmolarity set at $295 \mathrm{mOsm}$ ). This internal solution ensures that $G_{A B A}$ receptor activation leads to large depolarizations, allowing us to clearly measure mIPSCs. To prevent excitatory postsynaptic potentials, $50 \mu \mathrm{M}$ DL-AP5 (Tocris) and $20 \mu \mathrm{M}$ DNQX (Tocris) was included in the bath. To prevent spontaneous action potentials, I $\mu \mathrm{M}$ TTX (Abcam) was included in the bath. The pyramidal cell was kept in voltage clamp at $-60 \mathrm{mV}$, while mIPSCs were recorded for 2-5 minutes. Recordings were acquired using a Multiclamp 700B amplifier (Molecular Devices) and sampled at $20 \mathrm{kHz}$ using a Digidata I550a with HumSilencer (Molecular Devices). Cells were excluded when the initial resting membrane potential exceeded $-50 \mathrm{mV}$ or if Rs exceeded $30 \mathrm{M} \Omega$ before the experiment.

\section{Immunohistochemistry and confocal imaging}

Immunohistochemistry and confocal imaging was performed as described in chapter 3. The following primary antibodies were used: mouse(lgG2b) $\alpha-C B I R$ (Synaptic Systems 2580 I I; I:500), rabbit $\alpha$-VGAT (Synaptic Systems I3I 003; I:I000) and mouse(lgGI) $\alpha$-gephyrin (Synaptic Systems I 470 I I; I: I000). Secondary antibodies wereAlexa-405 $\alpha$-rabbit,Alexa-594 $\alpha$-mouse(lgG2b) and Alexa-647 $\alpha$-mouse(lgGI) conjugated goat antibodies (Invitrogen). Confocal image stacks were acquired with a Plan-Apochromat $63 \times 1.4$ NA oil immersion objective at 10 pixels per $\mu \mathrm{m}$ and with $0.3 \mu \mathrm{m}$ z-steps.

\section{Quantification and statistical analysis}

\section{Analysis of bouton dynamics, bouton growth and spine growth}

Analysis of the local application experiments was performed on median filtered images using the same custom written Matlab scripts as used in chapter 3 (Mathworks). All image analysis was done blind to the experimental conditions. One to four axons (average length $=38.5$ $\mu \mathrm{m}$ with $\mathrm{SD}=7.3 \mu \mathrm{m})$ within the field of view $(468 \times 468$ pixels, $51.5 \times 5 \mathrm{I} .5 \mu \mathrm{m})$ were analyzed. 
An axon threshold was set to separate the boutons from the axon, relative to the axon intensity (determined from the summed projection of 5 z-planes, modus along the entire axon) to account for possible intensity fluctuations between time points and differences between experiments. A box of $9 \times 9 \times 5$ voxels (i.e. $I \times I \times 2.5 \mu \mathrm{m}$ ) was positioned manually at each bouton. Bouton presence or absence was determined by the researcher, aided by the axon threshold calculated by the software. At bouton locations (axonal locations where boutons were present for at least 2 time points during the imaging period) where a bouton was absent, the location of the box was interpolated or extrapolated. Bouton presence was used to categorize boutons as persistent (P, present in all time points) or non-persistent (NP, not present in all, but at least in two time points) boutons. The non-persistent boutons were subsequently divided in new ( $\mathrm{N}$, absent before treatment, sometimes present after treatment), lost ( $L$, sometimes present before treatment, absent after treatment), stabilizing ( $\mathrm{S}$, sometimes present before treatment, always present after treatment), destabilizing (D, always present before treatment, sometimes present after treatment), and intermittent (I, sometimes present both before and after treatment). The number of boutons in each category was divided by the axon length to calculate bouton density. We considered all axonal swellings or varicosities as boutons. Boutons that appear only once during the imaging period were excluded, as they might reflect axonal varicosities that are not related to inhibitory synapses.

Next, we calculated a growth coefficient for each bouton location to quantify its growth or shrinkage during the experiment. The integrated intensity in the box around the bouton was calculated at each time point. The growth coefficient is defined as (V2 - VI)/(V2 + VI), in which $\mathrm{VI}$ is the average baseline bouton intensity and $\mathrm{V} 2$ is the maximal bouton intensity, determined over a sliding averaging window of 5 time points wide. A positive value of the growth coefficient corresponds with bouton growth, while a negative value corresponds with bouton shrinkage. As we also know the presence or absence of the boutons as determined by the researcher, a division between persistent and non-persistent boutons can be made. We further divided non-persistent boutons into two categories (low and high baseline intensity) using a baseline intensity of 20000 (arbitrary units) as an empirically determined threshold. This cutoff roughly divides the dataset into axon locations where no bouton is present in baseline ('low') and axon location where there is a bouton present in baseline ('high'). For the low baseline intensity boutons, we calculated the average intensity over time normalized to the baseline period.

For bath application experiments, bouton dynamics was quantified manually by the researcher using custom written Matlab scripts, as described previously (Frias et al., 2019; Schuemann et al., 2013). While different Matlab scripts were used to analyze local and bath application experiments, both methods use the same bouton category definitions and can therefore be compared.

Spine growth was analyzed as described in chapter 3. In brief: the integrated intensity of a box around the spine head was calculated for each time point. We then defined spine growth as the relative change in integrated intensity $(\Delta \% \mathrm{l})$, compared to the average intensity of the baseline period.

\section{Analysis of mIPSCs}

Electrophysiology recordings were analyzed using Clampfit 10.6. Recordings were first filtered using a Gaussian filter with a $-3 \mathrm{~dB}$ cutoff at $2 \mathrm{kHz}$. We selected the first minute of 
each recording for analysis. While some cells showed a substantial series resistance increase above $30 \mathrm{~m} \Omega$ after a 5 minute recording, these cells were included in the analysis and we assumed that for the first minute of the recording the series resistance was likely to be stable. We did not observe significant rundown of frequency or amplitude during the first minute. Next, automatic template detection was performed using a template that was created by averaging $>20$ mIPSCs in Gaussian filtered traces. The template match threshold was set at 2.4 and analysis was performed fully automated, meaning that all matches were accepted as true mIPSCs. We estimated that the false positive and false negative rate using the automated method was around $10 \%$. For each trace, the average amplitude and the frequency of mIPSCs was determined. For histograms, we randomly selected 50 mIPSCs of each cell and used their amplitude and inter-event interval.

\section{Analysis of confocal images}

Confocal images were analyzed using Fiji. To quantify bouton density, we selected up to 8 healthy-looking axons per stack. For each axon we manually counted putative boutons based on the GFP channel. We also looked at gephyrin colocalization in cases where it was unsure if a small swelling of the axon could be considered a bouton. The total number of boutons per axon was divided by the two-dimensional length of the axon to calculate bouton density.

To count colocalizations ofVGAT and gephyrin, the Fiji-plugin PunctaAnalyzer was used.After performing an average projection of five z-steps, up to four I0IxI0I pixel $(10.02 \times 10.02 \mu \mathrm{m})$ ROls with good quality staining were selected. Each ROI was analyzed as an independent measurement. PunctaAnalyzer was used to filter and threshold the images and puncta of sufficient size ( $>20$ pixels for VGAT, $>6$ pixels for gephyrin) were selected. A colocalization was defined as an overlap of a VGAT and a gephyrin punctum of at least one pixel.Any errors were corrected manually.

To quantify the fraction of CBIR+ axons, we performed maximum projections of 3-5 z-steps at up to three z-levels in an image stack. For each projection image, we manually counted every independent axonal stretch and classified them as CBIR+ or CBIR-. These numbers were added for the entire image stack, which was considered an independent measurement. We do not exclude that the same axons were occasionally counted twice at different z-levels, but this is unlikely to affect our results significantly.

\section{Statistics}

All data are presented as mean \pm SEM. Data was tested using a Mann-Whitney test unless otherwise indicated. We used a two-way ANOVA followed by a Sidak multiple comparisons test to compare growth coefficients of boutons with low and high baseline intensity, and to compare low baseline intensity bouton growth over time (Figure I). We used a one-way ANOVA followed by a Dunnett multiple comparisons test to compare bouton densities, synapse densities and the fraction of $\mathrm{CBIR+}$ axons (Figure 4). We used a two-way ANOVA followed by a Sidak multiple comparisons test to compare spine growth across all conditions (compare every condition with the stimulated condition, Figure 5). Statistical analyses were performed using Graphpad Prism 7. 


\section{Acknowledgements}

The authors would like to thank René van Dorland for excellent technical support and Balázs Rósza/Femtonics Ltd. for providing DNI-glutamate.

\section{Author Contributions}

DLHK designed, performed and analyzed all experiments, with the exception of the bath application experiments in Figure 2 which were performed by IGLMR and were analyzed by JL. DLHK designed all figures and wrote the manuscript. CJW was involved in the design of the study and supervised the project. 


\section{References}

Berghuis, P., Rajnicek, A. M., Morozov, Y. M., Ross, R. A., Mulder, J., Urban, G. M., et al. (2007). Hardwiring the Brain: Endocannabinoids Shape Neuronal Connectivity. Science 316, 1212-1216. doi: I0.1 I26/science. I I37406.

Bisogno, T., Berrendero, F., Ambrosino, G., Cebeira, M., Ramos, J. A., Fernandez-Ruiz, J. J., et al. (1999). Brain Regional Distribution of Endocannabinoids: Implications for Their Biosynthesis and Biological Function. Biochem. Biophys. Res. Commun. 256, 377-380. doi:10.1006/bbrc.1999.0254.

Buczynski, M. W., and Parsons, L. H. (20I0). Quantification of brain endocannabinoid levels: Methods, interpretations and pitfalls. Br. J. Pharmacol. I60, 423-442. doi:I0.IIII/j.I476538I.2010.00787.x.

Cabral, G.A., Ferreira, G.A., and Jamerson, M.J. (20I5). "Endocannabinoids and the Immune System in Health and Disease," in Endocannabinoids, ed. R. G. Pertwee (Springer International Publishing Switzerland), I85-2II. doi:DOI 10.1007/978-3319-20825-I_6.

Carlson, G., Wang, Y., and Alger, B. E. (2002). Endocannabinoids facilitate the induction of LTP in the hippocampus. Nat. Neurosci. 5, 723-724. doi:10.1038/nn879.

Castillo, P. E., Younts, T. J., Chávez, A. E., and Hashimotodani, Y. (20I2). Endocannabinoid Signaling and Synaptic Function. Neuron 76, 708I. doi:I0.10I6/j.neuron.2012.09.020.

Chávez, A. E., Chiu, C. Q., and Castillo, P. E. (20I0). TRPVI activation by endogenous anandamide triggers postsynaptic long-term depression in dentate gyrus. Nat. Neurosci. 13, I5II-1519. doi:10.1038/nn.2684.

Cheetham, C. E. J., Barnes, S. J., Albieri, G., Knott, G. W., and Finnerty, G. T. (20/4). Pansynaptic Enlargement at Adult Cortical Connections Strengthened by Experience. Cereb. Cortex 24, 52I-53I. doi: I0.1093/cercor/bhs334.

Chen, J. L., Villa, K. L., Cha, J. W., So, P. T. C., Kubota, Y., and Nedivi, E. (20I2). Clustered Dynamics of Inhibitory Synapses and Dendritic Spines in the Adult Neocortex. Neuron 74, 36I-373. doi:10.1016/j.neuron.2012.02.030.

Chevaleyre,V., and Castillo, P. E. (2003). Heterosynaptic LTD of Hippocampal GABAergic Synapses: A
Novel Role of Endocannabinoids in Regulating Excitability. Neuron 38, 46I-472. doi:10.1016/ s0896-6273(03)00235-6.

Chevaleyre, V., and Castillo, P. E. (2004). Endocannabinoid-Mediated Metaplasticity in the Hippocampus. Neuron 43,87I-88I. doi: 10.1016/j. neuron.2004.08.036.

Chevaleyre, V., Heifets, B. D., Kaeser, P. S., Südhof, T. C., and Castillo, P. E. (2007). EndocannabinoidMediated Long-Term Plasticity Requires cAMP/ PKA Signaling and RIMI $\alpha$. Neuron 54, 80I-8I2. doi:10.1016/j.neuron.2007.05.020.

Coutts, A. A., Anavi-Goffer, S., Ross, R. A., MacEwan, D. J., Mackie, K., Pertwee, R. G., et al. (200I). Agonist-Induced Internalization and Trafficking of Cannabinoid CBI Receptors in Hippocampal Neurons.J. Neurosci. 21, 2425-2433. doi:10.1523/ jneurosci.2I-07-02425.200I.

Cristino, L., de Petrocellis, L., Pryce, G., Baker, D., Guglielmotti, V., and Di Marzo, V. (2006). Immunohistochemical localization of cannabinoid type I and vanilloid transient receptor potential vanilloid type I receptors in the mouse brain. Neuroscience 139, 1405-1415. doi:10.1016/j. neuroscience.2006.02.074.

Cui, Y., Paillé, V., Xu, H., Genet, S., Delord, B., Fino, E., et al. (2015). Endocannabinoids mediate bidirectional striatal spike-timing-dependent plasticity. J. Physiol. 593, 2833-2849. doi: I0.1 I I 3/ JP270324.

Cui, Y., Prokin, I., Xu, H., Delord, B., Genet, S., Venance, L., et al. (2016). Endocannabinoid dynamics gate spike-timing dependent depression and potentiation. Elife 5, I-32. doi:10.7554/ eLife. 13185.

den Boon, F. S., Chameau, P., Schaafsma-Zhao, Q., van Aken,W., Bari, M., Oddi, S., et al. (20I2). Excitability of prefrontal cortical pyramidal neurons is modulated by activation of intracellular type-2 cannabinoid receptors. Proc. Natl. Acad. Sci. 109, 3534-3539. doi:10.1073/pnas. III8167109.

Dudok, B., Barna, L., Ledri, M., Szabó, S. I., Szabadits, E., Pintér, B., et al. (20I5). Cell-specific STORM super-resolution imaging reveals nanoscale organization of cannabinoid signaling. Nat. Neurosci. 18, 75-86. doi:I0.1038/nn.3892.

Frias, C. P., Liang, J., Bresser, T., Scheefhals, L., van Kesteren, M., van Dorland, R., et al. (2019). Semaphorin4D Induces Inhibitory Synapse Formation by Rapid Stabilization of Presynaptic 
Boutons via MET Coactivation. J. Neurosci. 39, 4221-4237. doi:10.1523/jneurosci.0215-19.2019.

Frias, C. P., and Wierenga, C. J. (20|3). Activitydependent adaptations in inhibitory axons. Front. Cell. Neurosci. 7, 219. doi:10.3389/ fncel.2013.00219.

Gaffuri, A. L., Ladarre, D., and Lenkei, Z. (20I2). Type-I Cannabinoid Receptor Signaling in Neuronal Development. Pharmacology 90, 19-39. doi:I0.II59/000339075.

Ganguly, A., Tang, Y., Wang, L., Ladt, K., Loi, J., Dargent, B., et al. (20I5). A dynamic formin-dependent deep F-actin network in axons. J. Cell Biol. 210, 40 I-4 I7. doi: I0.1083/jcb.20I506 I I0.

Harkany, T., Guzmán, M., Galve-Roperh, I., Berghuis, P., Devi, L. A., and Mackie, K. (2007). The emerging functions of endocannabinoid signaling during CNS development. Trends Pharmacol. Sci. 28, 8392. doi:10.1016/j.tips.2006.12.004.

Hashimotodani, Y., Ohno-Shosaku, T., Tsubokawa, H., Ogata, H., Emoto, K., Maejima, T., et al. (2005). Phospholipase $C \beta$ Serves as a Coincidence Detector through Its $\mathrm{Ca}^{2+}$ Dependency for Triggering Retrograde Endocannabinoid Signal. Neuron 45, 257-268. doi:10.1016/j. neuron.2005.01.004.

Heifets, B. D., and Castillo, P.E. (2009). Endocannabinoid Signaling and Long-Term Synaptic Plasticity. Annu. Rev. Physiol. 7I, 283-306. doi:10.1/46/annurev. physiol.010908.163149.

Heifets, B. D., Chevaleyre, V., and Castillo, P. E. (2008). Interneuron activity controls endocannabinoidmediated presynaptic plasticity through calcineurin. Proc. Natl.Acad. Sci. I05, I0250-10255. doi:10.1073/pnas.07|I880105.

Hsieh, C., Brown, S., Derleth, C., and Mackie, K. (1999). Internalization and Recycling of the CBI Cannabinoid Receptor. J. Neurochem. 73, 49350I. doi:I0.1046/j. I47|-4I59.1999.0730493.x.

Hu, S. S.-J., and Mackie, K. (20I5). “Distribution of the Endocannabinoid System in the Central Nervous System," in Endocannabinoids, ed. R. G. Pertwee (Springer International Publishing Switzerland), 59-93. doi:DOI I0.1007/978-3-3I9-20825-I_3.

Jin,W.,Brown,S., Roche,J.P.,Hsieh,C., Celver,J.P., Kovoor, A., et al. (1999). Distinct Domains of the CBI Cannabinoid Receptor Mediate Desensitization and Internalization. J. Neurosci. 19, 3773-3780. doi: 10.1523/jneurosci. 19-10-03773.1999.
Jung, K.-M., Astarita, G., Zhu, C., Wallace, M., Mackie, K., and Piomelli, D. (2007). A Key Role for Diacylglycerol Lipase- $\alpha$ in Metabotropic Glutamate Receptor-Dependent Endocannabinoid Mobilization. Mol. Pharmacol. 72, 6I2-62 I. doi: |0. I | 24/mol. I07.037796.

Kano, M., Ohno-Shosaku, T., Hashimotodani, Y., Uchigashima, M., and Watanabe, M. (2009). Endocannabinoid-mediated control of synaptic transmission. Physiol. Rev. 89, 309-80. doi:I0.II52/ physrev.000I9.2008.

Katona, I., Sperlágh, B., Sik, A., Käfalvi, A., Vizi, E. S., Mackie, K., et al. (1999). Presynaptically Located CBI Cannabinoid Receptors Regulate GABA Release from Axon Terminals of Specific Hippocampal Interneurons.J. Neurosci. 19,45444558. doi: 10. I523/jneurosci. I9- I I-04544. 1999.

Katona, I., Urbán, G. M., Wallace, M., Ledent, C., Jung, K.-M., Piomelli, D., et al. (2006). Molecular Composition of the Endocannabinoid System at Glutamatergic Synapses. J. Neurosci. 26, 56285637. doi:I0.I523/JNEUROSCI.0309-06.2006.

Kawamura,Y.,Fukaya,M.,Maejima,T.,Yoshida,T.,Miura, E., Watanabe, M., et al. (2006). The CBI Cannabinoid Receptor Is the Major Cannabinoid Receptor at Excitatory Presynaptic Sites in the Hippocampus and Cerebellum. J. Neurosci. 26, 299I-300I. doi:I0.1523/JNEUROSCI.4872-05.2006.

Kreitzer, A. C., and Regehr,W. G. (200I). Retrograde Inhibition of Presynaptic Calcium Influx by Endogenous Cannabinoids at Excitatory Synapses onto Purkinje Cells. Neuron 29, 717727. doi:10.1016/S0896-6273(0I)00246-X.

$\mathrm{Li}, \mathrm{Y}$. , and Kim, J. (20I5). Neuronal expression of CB2 cannabinoid receptor mRNAs in the mouse hippocampus. Neuroscience 31I, 253-267. doi:I0.1016/j.neuroscience.2015.10.04I.

López-Bendito, G., Sturgess, K., Erdélyi, F., Szabó, G., Molnár, Z., and Paulsen, O. (2004). Preferential Origin and Layer Destination of GAD65-GFP Cortical Interneurons. Cereb. Cortex 14, II22I I33. doi:I0.1093/cercor/bhh072.

Lu, H.-C., and Mackie, K. (20I6).An introduction to the endogenous cannabinoid system. Biol. Psychiatry 79, 516-525. doi:10.1016/j.biopsych.2015.07.028.

Maroso, M., Szabo, G. G., Kim, H. K., Alexander, A., Bui, A. D., Lee, S.-H., et al. (20I6). Cannabinoid Control of Learning and Memory through HCN Channels. Neuron 89, 1059-1073. doi:10.1016/j. neuron.2016.01.023. 
Marsicano, G., and Lutz, B. (1999). Expression of the cannabinoid receptor $\mathrm{CBI}$ in distinct neuronal subpopulations in the adult mouse forebrain. Eur. J. Neurosci. II, 4213-4225. doi:10.1046/j.14609568.1999.00847.x.

Meyer, D., Bonhoeffer, T., and Scheuss, V. (2014). Balance and Stability of Synaptic Structures during Synaptic Plasticity. Neuron 82, 430-443. doi:I0.10I6/j.neuron.2014.02.03I.

Monory, K., Polack, M., Remus, A., Lutz, B., and Korte, M. (20I5). Cannabinoid CBI Receptor Calibrates Excitatory Synaptic Balance in the Mouse Hippocampus. J. Neurosci. 35, 3842-3850. doi:I0.1523/JNEUROSCI.3I67-|4.20I5.

Murakoshi, H., Wang, H., and Yasuda, R. (20I I). Local, persistent activation of Rho GTPases during plasticity of single dendritic spines. Nature 472 , 100-106. doi:10.1038/nature09823.

Murataeva, N., Straiker, A., and MacKie, K. (2014). Parsing the players: 2-arachidonoylglycerol synthesis and degradation in the CNS. Br. J. Pharmacol. |7|, |379-|39|. doi:I0.IIII/ bph. I24II.

Murthy, V. N., Schikorski, T., Stevens, C. F., and Zhu, Y. (200I). Inactivity produces increases in neurotransmitter release and synapse size. Neuron 32, 673-682. doi:10.1016/S08966273(0I)00500-I.

Navarrete, M., and Araque,A. (2008). Endocannabinoids Mediate Neuron-Astrocyte Communication. Neuron 57, 883-893. doi:10.1016/j. neuron.2008.01.029.

Navarrete, M., Díez, A., and Araque, A. (2014). Astrocytes in endocannabinoid signalling. Philos. Trans. R. Soc. B Biol. Sci. 369. doi:I0.1098/ rstb.20I3.0599.

Njoo, C., Agarwal, N., Lutz, B., and Kuner, R. (20I5). The Cannabinoid Receptor CBI Interacts with the WAVEI Complex and Plays a Role in Actin Dynamics and Structural Plasticity in Neurons. PLoS Biol. 13, I-36. doi: I 0.1371/journal. pbio. 1002286.

Nyíri, G., Cserép, C., Szabadits, E., Mackie, K., and Freund, T. F. (2005). CBI cannabinoid receptors are enriched in the perisynaptic annulus and on preterminal segments of hippocampal GABAergic axons. Neuroscience 136, 81 I-822. doi:10.1016/j.neuroscience.2005.01.026.

Ohno-Shosaku, T., Maejima, T., and Kano, M. (200I).
Endogenous Cannabinoids Mediate Retrograde Signals from Depolarized Postsynaptic Neurons to Presynaptic Terminals. Neuron 29, 729-738. doi:I0.1016/S0896-6273(0I)00247-I.

Ohno-Shosaku,T.,Tsubokawa, H., Mizushima, I.,Yoneda, N., Zimmer, A., and Kano, M. (2002). Presynaptic Cannabinoid Sensitivity Is a Major Determinant of Depolarization-Induced Retrograde Suppression at Hippocampal Synapses. J. Neurosci. 22, 38643872. doi: 10.1523/jneurosci.22-10-03864.2002.

Papandréou, M. J., and Leterrier, C. (2018). The functional architecture of axonal actin. Mol. Cell. Neurosci. 91, 15I-159. doi:10.1016/j. mcn.2018.05.003.

Pierce, J. P., and Lewin, G. R. (1994). An ultrastructural size principle. Neuroscience 58, 44I-446. doi:I0.1016/0306-4522(94)9007I-X.

Piomelli, D. (20|4). More surprises lying ahead. The endocannabinoids keep us guessing. Neuropharmacology 76, 228-234. doi:10.1016/j. neuropharm.2013.07.026.

Richardson, D., Ortori, C.A., Chapman,V., Kendall, D.A., and Barrett, D. A. (2007). Quantitative profiling of endocannabinoids and related compounds in rat brain using liquid chromatography-tandem electrospray ionization mass spectrometry. Anal. Biochem. 360, 216-226. doi:10.1016/j. ab.2006.10.039.

Roland, A. B., Ricobaraza, A., Carrel, D., Jordan, B. M., Rico, F., Simon, A., et al. (20/4). Cannabinoidinduced actomyosin contractility shapes neuronal morphology and growth. Elife 3, I-23. doi:I0.7554/elife.03I59.

Schuemann, A., Klawiter, A., Bonhoeffer, T., and Wierenga, C. J. (20|3). Structural plasticity of GABAergic axons is regulated by network activity and GABAA receptor activation. Front. Neural Circuits 7, II3. doi:10.3389/fncir.2013.001।3.

Shonesy, B. C., Winder, D. D. G., Patel, S., and Colbran, R. R. J. (20I5). The initiation of synaptic 2-AG mobilization requires both an increased supply of diacylglycerol precursor and increased postsynaptic calcium. Neuropharmacology 91, 57-62. doi: I0.1016/j.neuropharm.2014.II.026.

Sjöström, P.J.,Turrigiano, G. G., and Nelson, S. B. (2003). Neocortical LTD via Coincident Activation of Presynaptic NMDA and Cannabinoid Receptors. Neuron 39, 64I-654. doi:10.1016/s08966273(03)00476-8. 
Staras, K. (2007). Share and share alike: trading of presynaptic elements between central synapses. Trends Neurosci. 30, 292-298. doi:10.1016/j. tins.2007.04.005.

Staras, K., Branco, T., Burden, J. J., Pozo, K., Darcy, K., Marra,V., et al. (2010). A Vesicle Superpool Spans Multiple Presynaptic Terminals in Hippocampal Neurons. Neuron 66, 37-44. doi:10.1016/j. neuron.2010.03.020.

Stella, N., Schweitzer, P., and Plomelli, D. (1997). A second endogenous cannabinoid that modulates long-term potentiation. Nature 388, 773-778. doi:10.1038/420I5.

Stempel, A. V., Stumpf, A., Zhang, H.-Y., Özdoğan, T., Pannasch,U.,Theis,A.-K.,etal.(20I6). Cannabinoid Type 2 Receptors Mediate a Cell Type-Specific Plasticity in the Hippocampus. Neuron 90, 795809. doi:10.1016/j.neuron.2016.03.034.

Stoppini, L., Buchs, P.-A., and Muller, D. (1991). A simple method for organotypic cultures of nervous tissue. J. Neurosci. Methods 37, I73-182. doi:10.1016/0165-0270(91)90I28-M.

Thibault, K., Carrel, D., Bonnard, D., Gallatz, K., Simon, A., Biard, M., et al. (20।3). Activation-Dependent Subcellular Distribution Patterns of CBI Cannabinoid Receptors in the Rat Forebrain. Cereb. Cortex 23, 258I-259I. doi:I0.1093/cercor/ bhs240.

Tóth,A., Boczán, J., Kedei, N., Lizanecz, E., Bagi, Z., Papp, Z., et al. (2005). Expression and distribution of vanilloid receptor I (TRPVI) in the adult rat brain. Mol. Brain Res. 135, 162-168. doi:10.1016/j. molbrainres.2004.12.003.

Tsou, K., Mackie, K., Sañudo-Peña, M. C., and Walker J. M. (1999). Cannabinoid CBI receptors are localized primarily on cholecystokinincontaining GABAergic interneurons in the rat hippocampal formation. Neuroscience 93, 969975. doi: I0. I016/S0306-4522(99)00086-X.

Villa, K. L., Berry, K. P., Subramanian, J., Cha, J.W., Oh,W. C., Kwon, H.-B., et al. (20I6). Inhibitory Synapses Are Repeatedly Assembled and Removed at Persistent Sites In Vivo. Neuron 89, 756-769. doi:10.1016/j.neuron.2016.01.010.

Wang, W., Jia, Y., Pham, D. T., Palmer, L. C., Jung, K.-M., Cox, C. D., et al. (2018). Atypical Endocannabinoid Signaling Initiates a New Form of Memory-Related Plasticity at a Cortical Input to Hippocampus. Cereb. Cortex 28, 2253-2266. doi:I0.1093/cercor/bhx| 26
Wang, W., Trieu, B. H., Palmer, L. C., Jia, Y., Pham, D. T., Jung, K.-M., et al. (2016). A Primary Cortical Input to Hippocampus Expresses a PathwaySpecific and Endocannabinoid-Dependent Form of Long-Term Potentiation. eNeuro 3, 160-16. doi:I0.1523/ENEURO.0160-16.2016.

Wierenga, C.J. (20I7). Live imaging of inhibitory axons: Synapse formation as a dynamic trial-and-error process. Brain Res. Bull. 129, 43-49. doi:10.1016/j. brainresbull.2016.09.018.

Wierenga, C. J., Becker, N., and Bonhoeffer, T. (2008). GABAergic synapses are formed without the involvement of dendritic protrusions. Nat. Neurosci. II, 1044-1052. doi:10.1038/nn.2180.

Wierenga, C. J., Müllner, F. E., Rinke, I., Keck, T., Stein, V., and Bonhoeffer, T. (20I0). Molecular and Electrophysiological Characterization of GFPExpressing CAI Interneurons in GAD65-GFP Mice. PLoS One 5, I-II. doi:I0.137I/journal. pone.00159I5.

Wilson, R. I., and Nicoll, R. A. (200I). Endogenous cannabinoids mediate retrograde signalling at hippocampal synapses. Nature 410, 588-592. doi:10.1038/35069076.

Wu, D.-F., Yang, L.-Q., Goschke, A., Stumm, R., Brandenburg, L.-O., Liang, Y.-J., et al. (2008). Role of receptor internalization in the agonist-induced desensitization of cannabinoid type I receptors.J. Neurochem. 104, I I32-I |43. doi: I0.1 I I I/j. I47I4I59.2007.05063.x.

Xu, J.-Y., Zhang, J., and Chen, C. (20/2). Longlasting potentiation of hippocampal synaptic transmission by direct cortical input is mediated via endocannabinoids. J. Physiol. 590, 2305-23I5. doi:I0.1 I I3/jphysiol.20 I I.2235 I I.

Xu, J., Antion, M. D., Nomura, T., Kraniotis, S., Zhu, Y., and Contractor, A. (20/4). Hippocampal Metaplasticity Is Required for the Formation of Temporal Associative Memories. J. Neurosci. 34, 16762-16773. doi:10.1523/ jneurosci.2869-|3.20|4.

Yasuda, H., Huang,Y., and Tsumoto,T. (2008). Regulation of excitability and plasticity by endocannabinoids and PKA in developing hippocampus. Proc. Natl. Acad. Sci. 105, 3106-31।I. doi:10.1073/ pnas.0708349I05.

Yoshida, T., Fukaya, M., Uchigashima, M., Miura, E., Kamiya, H., Kano, M., et al. (2006). Localization of Diacylglycerol Lipase- $\alpha$ around Postsynaptic Spine Suggests Close Proximity between 
Production Site of an Endocannabinoid, 2-Arachidonoylglycerol, and Presynaptic Cannabinoid CBI Receptor.J. Neurosci. 26,4740475I. doi:I0.I523/JNEUROSCI.0054-06.2006.

Younts, T. J., and Castillo, P. E. (20/4). Endogenous cannabinoid signaling at inhibitory interneurons. Curr. Opin. Neurobiol. 26, 42-50. doi:10.1016/j. conb.20I3.I2.006.

Younts, T. J., Monday, H. R., Dudok, B., Klein, M. E., Jordan, B. A., Katona, I., et al. (2016). Presynaptic Protein Synthesis Is Required for Long-Term Plasticity of GABA Release. Neuron 92, 479-492. doi:I0.1016/j.neuron.2016.09.040. 


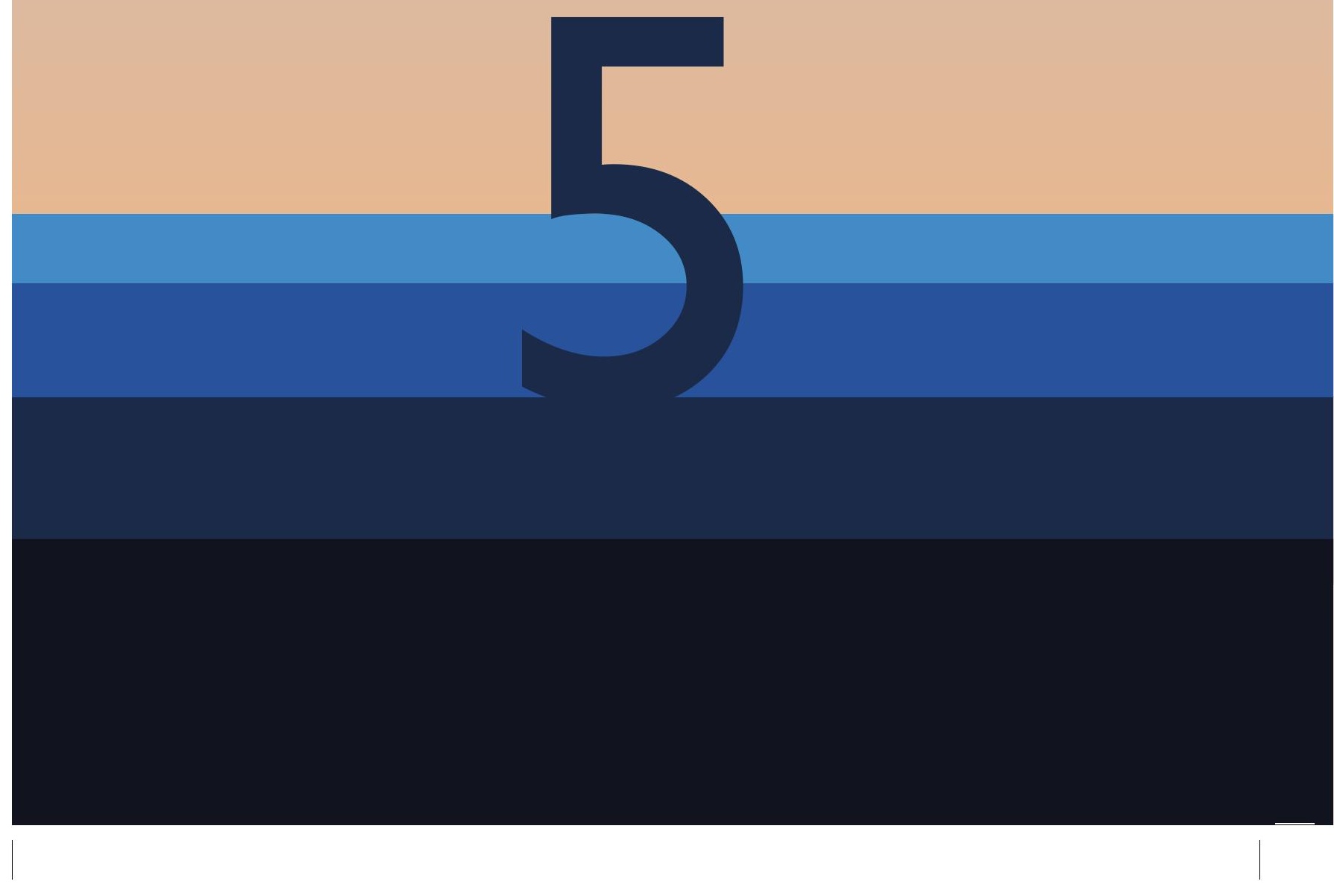




\title{
General discussion
}

\author{
Dennis L. H. Kruijssen
}


To understand the ever-changing brain, we need to understand how connections in the brain are formed and by what rules they change. With this thesis, I aimed to unravel a few puzzle pieces in the giant puzzle of synapse formation, synaptic plasticity and excitatory/inhibitory coordination. In chapter 2, I summarized the literature on excitatory long-term potentiation on the level of the single synapse. I argued that single synapse LTP is dependent on context, as experimental conditions as well as interactions with the dendritic vicinity can have a strong effect on the expression of LTP. In chapter 3, we showed that local perturbations of the coordination of excitation and inhibition can rapidly be adjusted. After stimulating and potentiating a small cluster of excitatory synapses, an inhibitory presynaptic bouton grew nearby and this process was mediated by endocannabinoid signaling. As endocannabinoids were mainly known for mediating the depression of inhibitory synapses, I followed up this surprising effect on bouton growth in chapter 4. I presented preliminary data suggesting that brief exposure to endocannabinoids can promote bouton growth on short time scales and inhibitory synapse formation on longer time scales. Additionally, manipulations of the endocannabinoid system seem to affect LTP of single excitatory synapses. Together, these results paint a picture of the many complex ways the neural network can be regulated on a local level.

\section{What does it take to form an inhibitory synapse?}

It is not yet fully understood what it takes to form an inhibitory synapse. We know that inhibitory synapses are highly dynamic structures and typically form in a pre-before-post manner (Dobie and Craig, 20I I; Frias et al., 2019; Wierenga et al., 2008). To study the first steps of inhibitory synapse formation, it is therefore useful to look at inhibitory boutons. What triggers the growth of a presynaptic bouton? In chapter 3, we presented an endocannabinoidmediated mechanism that can trigger inhibitory bouton growth as a response to a local change in excitation. But what triggers endocannabinoid release? And how is subsequent bouton growth regulated?

\section{Endocannabinoid-mediated inhibitory synapse formation}

In chapter 3 we showed that inhibitory bouton growth after excitatory synapse stimulation is mediated by the endocannabinoid 2-AG, as bouton growth did not occur when synthesis of 2-AG by DAGL is prevented. DAGL activity is thus required, but we did not yet elucidate how DAGL activity is triggered by glutamate uncaging at excitatory synapses. DAGL activity is typically dependent on the local calcium concentration as well as the availability of the precursor DAG (Hashimotodani et al., 2005; Heifets and Castillo, 2009; Jung et al., 2007; Murataeva et al., 2014; Shonesy et al., 20I5; Stella et al., 1997). We showed that inhibitory bouton growth does not occur when the uncaging stimulus is applied in the presence of an NMDA receptor antagonist. This suggests that calcium inflow through NMDA receptors provides the increase in calcium required to activate DAGL (Figure I). However, recently nonionotropic signaling through NMDA receptors has been observed (Dore et al., 20 I6; Nabavi et al., 20I3; Stein et al., 20I5). As the pharmacological inhibitor used in our experiments prevents NMDA receptor activation altogether, we cannot exclude a metabotropic role for the NMDA receptor. Performing experiments with pharmacological blockers of the NMDA receptor ion pore should allow us to distinguish between the role of ionotropic and metabotropic NMDA receptor signaling.

The production of DAG can be stimulated by activation of PLC through group I metabotropic 

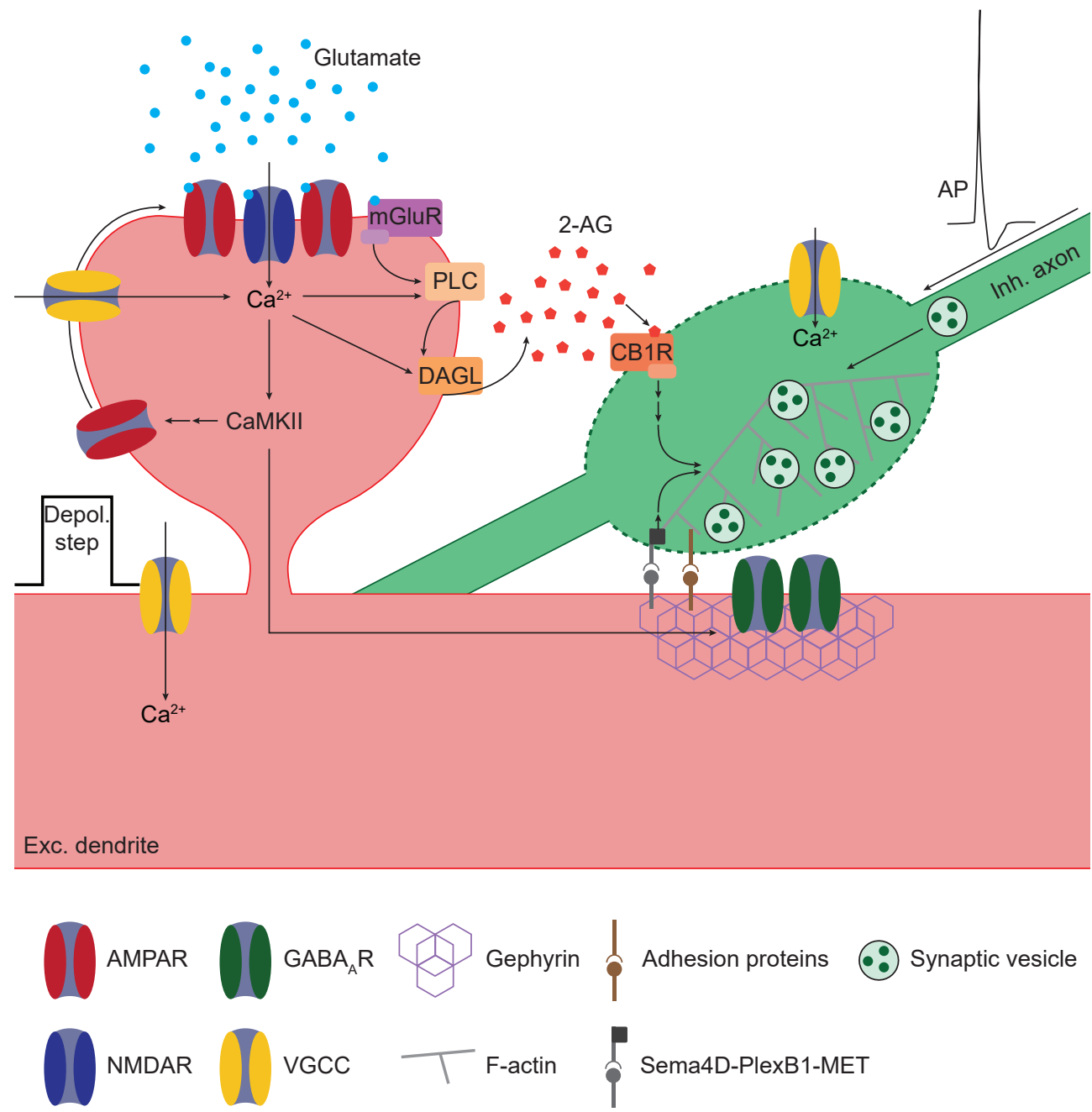

Figure 1: Speculative model of the signaling pathways involved in endocannabinoid-mediated inhibitory bouton growth and synapse formation

Glutamate uncaging is paired with a postsynaptic depolarizing step to activate NMDA receptors in the dendritic spine.This leads to an influx of calcium. The depolarizing step likely activates voltage-gated calcium channels (VGCCs) in the dendrite and spine as well. Glutamate uncaging also activates AMPA receptors and group I metabotropic glutamate receptors (mGluRs). mGluRs activation and the increase in intracellular calcium lead to the activation of phospholipase C (PLC), which produces diacylglycerol (DAG). DAG and calcium activate diacylglycerol lipase (DAGL), which produces the endocannabinoid 2-AG. The CBI receptor in the inhibitory axon is activated by 2-AG and this leads to a signaling cascade resulting in the remodeling of actin, the trapping of synaptic vesicles and bouton growth. Action potentials and the activation of VGCCs in the inhibitory axon might be required for this process. The calcium influx in the dendritic spine also activates $\mathrm{Ca}^{2+} /$ calmodulin kinase II (CaMKII). CaMKII activation, together with other signaling pathways, triggers a signaling pathway that leads to an increase in surface AMPA receptors at the excitatory synapse (long-term potentiation). It has been shown before that CaMKII activation can affect gephyrin clustering and $G A B A_{A} R$ recruitment, providing a potential mechanism for inhibitory postsynapse formation. The postsynapse and presynapse are coordinated by several adhesion proteins. Our lab has previously shown that signaling through Sema4D-PlexinBI-MET causes actin remodeling in the bouton, leading to bouton stabilization. 
glutamate receptors (Hashimotodani et al., 2005; Heifets and Castillo, 2009; Jung et al., 2007; Murataeva et al., 2014; Shonesy et al., 2015; Stella et al., 1997). These receptors are typically situated perisynaptically (Scheefhals and MacGillavry, 2018) and are therefore likely to be activated by our glutamate uncaging stimulus (Figure I). To confirm the involvement of mGluRs, we could repeat our uncaging experiments in presence of an mGluR antagonist, which is expected to prevent inhibitory bouton growth. During physiological synapse activation, spillover of glutamate activates perisynaptic mGluRs and activation is likely much stronger at higher frequencies and prolonged activity (Reiner and Levitz, 20I8; Viaene et al., 20I3). Involvement of mGluR signaling would therefore suggest that endocannabinoidmediated bouton growth is robustly triggered when a local cluster of synapses is particularly strongly activated. This would make sense in the context of E/l coordination: strong local excitatory activation indicates a local imbalance, justifying the formation of the inhibitory synapse.

The endocannabinoids that are released from the dendrite activate $C B I$ receptors, which are presumably on the inhibitory axon. In chapter 3 and chapter 4 , we showed that preventing CBIR activation prevents bouton growth. Many inhibitory axons strongly express the CBI receptor, but we have yet to formally show that it is these $\mathrm{CBI}$ receptors (and for example not the receptors on astrocytes (Navarrete et al., 20I4; Navarrete and Araque, 2008)) that need to be activated (Figure I). CBI receptors are G-protein coupled receptors and can activate many downstream pathways. While the CBI receptor is typically coupled to $\mathrm{G}_{\mathrm{i} / \mathrm{o}}$, coupling to other G-proteins has also been observed (Berghuis et al., 2007; Heifets and Castillo, 2009; Roland et al., 20I4). Major questions that remain are through which G-protein bouton formation is regulated and what downstream pathways are involved.

The bouton is rich in actin (Ganguly et al., 20I5; Papandréou and Leterrier, 2018) and we therefore believe that changes in the actin balance could be an essential step in bouton formation. Previous work from our lab has shown that the adhesion molecule Sema4D signals through PlexinBI and its co-receptor MET to change actin dynamics in the presynapse (Frias et al., 2019). Although this process occurs during bouton stabilization, it is likely that changes in actin dynamics are also required for the earlier steps of bouton formation that are discussed in this thesis. Vesicle recruitment is an early step in synapse formation, and actin meshworks might play a role in the capturing and positioning of presynaptic vesicles, that are known to be exchanged between presynapses (Cingolani and Goda, 2008; Dobie and Craig, 20I I; Frias and Wierenga, 20I3) (Figure I).

A curious observation we did in chapter 3 is that when LTP was induced using a magnesiumfree protocol, this did not lead to inhibitory bouton growth. As discussed in chapter 2, magnesium-free protocols and paired protocols are not per se interchangeable: they might activate different downstream signaling pathways, which could explain our observation. However, an interesting alternative cause for this difference is the presence of TTX in the magnesium-free condition. This drug is added to prevent aberrant plasticity in absence of magnesium (as is typically done for magnesium-free glutamate uncaging, see chapter 2 and (Harward et al., 20l6; Matsuzaki et al., 2004; Tanaka et al., 2008)) and blocks all action potential activity in the slice. The absence of bouton growth in presence of TTX might therefore indicate a requirement for presynaptic action potential activity. Inhibitory boutons are sensitive to changes in activity. In vivo, retinal lesion leads to a rapid loss of inhibitory boutons in the visual cortex (Keck et al., 20 I I). Our lab has previously shown that prolonged inhibition of activity in slices by TTX reduces bouton dynamics (Schuemann et al., 2013) and acute treatment with TTX can interfere with Sema4D-induced bouton stabilization 
(Frias et al., 2019). It needs to be noted that it is not possible to distinguish between preand postsynaptic activity in these experiments. Furthermore, endocannabinoid-mediated presynaptic LTD is thought to require not only $\mathrm{CBI}$ receptor activation but also electrical activity in the presynapse (Heifets et al., 2008). It will be interesting to further look into the role of presynaptic activity in inhibitory bouton formation, especially when mediated by endocannabinoid signaling from the dendrite (Figure I). In the latter situation, presynaptic activation combined with simultaneous dendrite-mediated endocannabinoid signaling would provide specificity: a bouton will only form on an axon that is electrically active in the same time frame as the stimulated excitatory synapses, increasing the likelihood that the new bouton will be able to exert the proper inhibitory control on the dendritic segment.

To address the activity dependence of bouton growth, several experiments can be performed. First, the uncaging experiment from chapter 3 and the local endocannabinoid application experiment from chapter 4 could be repeated in the presence of TTX. This would allow us to measure if action potentials are required for these mechanisms anywhere in the slice. To assess the contribution of activity in the presynaptic neuron specifically, we could perform these experiments after expressing excitability modulators (such as DREADDs or constitutively active potassium channels (Roth, 2016; Wiegert et al., 2017)) in inhibitory neurons. Alternatively, these experiments could be performed using optogenetic tools (Channelrhodopsin and Archaerhodopsin variants, (Fenno et al., 20II)) to acutely induce or suppress presynaptic activity. These last experiments would also allow us to study during what time window presynaptic activity is required.

\section{Additional signals needed for inhibitory synapse maturation?}

Inhibitory synapse formation is a multi-step process that likely requires more than a single signal to be completed. Endocannabinoid signaling upon excitatory synapse stimulation promotes bouton growth, but this will not always be sufficient to induce formation of a functional inhibitory synapse. This is reflected in the highly dynamic behavior of bouton growth: many newly formed boutons continued to behave dynamically and some had even disappeared again by the end of the imaging period (see chapter 3 ). The continuous challenge of understanding inhibitory synapse formation is to understand what other signals are required and how these signals cooperate. Previous work from our lab has shown that Sema4D can stabilize dynamic boutons through PlexinBI-MET signaling and actin remodeling (Frias et al., 2019). It would be interesting to see if the boutons that grow upon excitatory synapse stimulation are more likely to persist after Sema4D application.

To form a functional synapse, a postsynapse needs to be formed opposite to the bouton. This likely involves many additional signals that regulate the recruitment of scaffolding proteins and GABA receptors, as well as adhesion proteins that interact across the synaptic cleft. To study these processes, visualizing these putative inhibitory synapse regulators, such as neuroligin-2 and gephyrin, with live fluorescent labels is needed. By studying the dynamics of these markers, we could dissect in what order and on what time scales they arrive and how they contribute to forming the synapse. These experiments will be essential for understanding the fine molecular details of inhibitory synapse formation. 


\section{How are excitation and inhibition coordinated locally?}

\section{Endocannabinoids as crucial local coordinators of excitation and inhibition?}

Endocannabinoids are well known for mediating short- and long-term depression of inhibitory synapses (see chapter 4 and (Castillo, 20I2)). However, the eCB system turns out to be much more complex. Endocannabinoids can be involved in depolarization-induced suppression of excitation (Kreitzer and Regehr, 200I; Lu and Mackie, 20I6) and it has recently been shown that endocannabinoids can also mediate excitatory LTP (Cui et al., 2015, 2016; Wang et al., 20I6; Xu et al., 20I2). In this thesis we have presented a novel role for endocannabinoids in inhibitory synapse formation (chapter 3 and chapter 4) and preliminary data suggests that they can also affect the expression of single synapse LTP (chapter 4). Altogether, these different observations paint a complicated picture of bidirectional endocannabinoid-mediated effects on excitatory as well as inhibitory synapses. Depending on the mechanisms that are activated in a certain brain region, cell type, or even individual synapses, endocannabinoid signaling could locally disturb or restore and maintain E/I coordination. Further research is needed to understand what mechanisms are triggered under which circumstances, and how important eCB signaling is for local E/l coordination.

The timing of endocannabinoid signaling will likely play an important role in deciding what mechanisms are triggered. Short- and long-term depression of inhibitory synapses is regulated by distinct mechanisms, and which mechanism is activated depends on the duration of CBI receptor activation (Castillo, 20I2; Chevaleyre and Castillo, 2003). A study in the striatum has shown that the pattern and duration of endocannabinoid release determines whether excitatory LTD or LTP is triggered (Cui et al., 2016). On a longer time scale, we showed in chapter 4 that prolonged or brief stimulation of the eCB system lead to opposite effects. It would be interesting to acquire precise spatial and temporal control of CBIR activation, perhaps through a caged endocannabinoid or a optogenetically activatable GPCR (Heinbockel et al., 2005; Laguerre et al., 2019; Spangler and Bruchas, 2017), and thereby dissect the dynamic aspects of eCB signaling.

\section{Other mechanisms to locally coordinate excitation and inhibition?}

The mechanisms described in this thesis could play an important role in regulating inhibitory synapse formation and E/I coordination. Endocannabinoids are central to these mechanisms, but what about inhibitory neurons that do not seem to express endocannabinoid receptors? Currently, we cannot distinguish between $\mathrm{CBI}$ receptor positive and negative inhibitory axons in our slices during live imaging experiments. We therefore do not know whether excitatory synaptic stimulation also triggers bouton growth in axons that do not express the CBI receptor. This issue could be addressed by generating GFP-CBI receptor knockins in inhibitory axons, or by using transgenic mice that have fluorescently labelled CBI receptor-positive inhibitory neurons. This way, we can determine if the likelihood of inducing bouton growth is higher when monitoring $\mathrm{CBI}$ receptor-positive axons exclusively. It is however important to realize that the situation may not be so black-and-white. While our immunohistochemistry experiments show that roughly half of the GFP-labeled inhibitory axons in our organotypic slices also stain positive for the CBI receptor, it is possible that 
other inhibitory axons express the receptor at levels that are undetectable. Furthermore, astrocytes can express the $C B I$ receptor as well, and could therefore mediate indirect effect of endocannabinoids on neurons that do not express the receptor (Navarrete et al., 2014; Navarrete and Araque, 2008).

It is however likely that axons that express the $\mathrm{CBI}$ receptor at low levels, or not at all, use other mechanisms to coordinate inhibitory bouton growth. Several other mechanisms have the potential to locally link excitatory and inhibitory plasticity. For example, a recent study by Chiu and colleagues showed that activation of NMDA receptors potentiates synapses of a specific subtype of dendritically targeted inhibitory neurons, leaving other neuronal subtypes unaffected. Unlike the mechanism we reported in chapter 3, this inhibitory LTP was coordinated completely postsynaptically through GABA receptors (Chiu et al., 2018). Several other studies have suggested similar interactions between NMDA receptor activation and gephyrin clusters or GABA receptors (Marsden et al., 2007, 20I0; Petrini et al., 2014). While this type of mechanism has the potential to occur locally, this has not formally been shown, as many studies use global NMDA receptor activation to initiate this form of inhibitory plasticity. Additionally, these studies observe a strengthening of existing inhibitory synapses, while our results in chapter 3 are in agreement with the formation of new inhibitory synapses. Future experiments could show if and how mechanisms that affect the inhibitory presynapse and mechanisms that affect the inhibitory postsynapse are triggered in parallel to coordinate synapse formation and plasticity from both sides.

\section{Is inhibitory synapse formation and plasticity always coordinated by excitation?}

One of the main conclusions of this thesis is that inhibitory synapse formation can locally be coordinated by excitatory synaptic activity. Similarly, several forms of inhibitory synaptic plasticity are heterosynaptic: they are dependent on the activity of excitatory synapses nearby (Castillo et al., 20I I). This leads to wonder if inhibitory synaptic plasticity can also be regulated without the involvement of excitation. Many plasticity mechanisms require an increase in calcium in the postsynapse, which is a direct consequence of excitatory synapse activation, but not inhibitory synapse activation. Recently, a study showed that pairing inhibitory synapse activation with postsynaptic action potentials can lead to calcium influx and plasticity without the involvement of excitatory synapses. The hyperpolarization caused by inhibitory synapse activation leads to de-inactivation of voltage-gated calcium channels (VGCCs) in the postsynaptic neuron, and these VGCCs are subsequently activated by a backpropagating action potential. Interestingly, this leads to LTD at perisomatic inhibitory synapses, but to LTP at dendritic inhibitory synapses (Udakis et al., 2019). Similar homosynaptic inhibitory plasticity mechanisms that indirectly trigger calcium influx might be elucidated in the near future. Alternatively, calcium might not be necessary to trigger homosynaptic inhibitory plasticity. Perhaps the activation of metabotropic GABA ${ }_{B}$ receptors could trigger signaling pathways that homosynaptically modulate the inhibitory synapse. Twophoton GABA uncaging experiments (see below) could be used to explore the concept of homosynaptic inhibitory plasticity at the single synapse level.

The mechanisms discussed in this thesis could potentially be triggered by other signals than excitatory synapse activation. As discussed above, endocannabinoid synthesis is dependent on the availability of DAG, which is synthesized upon GPCR-mediated activation of PLC. This means that not only group I mGluRs, but many other $\mathrm{G}_{\mathrm{q}}$-coupled GPCRs can trigger 
or modulate endocannabinoid synthesis and thereby inhibitory synaptic plasticity. For example, neuromodulatory neurotransmitters such as dopamine and acetylcholine activate $\mathrm{G} \alpha$-coupled GPCRs and could therefore mobilize endocannabinoids (Giuffrida et al., 1999; Heifets and Castillo, 2009; Kim et al., 2002). This way, formation and plasticity of inhibitory synapses could occur without the involvement of excitatory synapses.

\section{A tight coordination of excitation and inhibition}

We showed that stimulating and strengthening excitatory synapses can cause a local increase in inhibition. When we think of these results in terms of E/l coordination, it automatically raises the question if opposite mechanisms are in place as well. For example, when excitation is reduced locally, could this lead to the weakening or removal of a inhibitory synapse nearby? This question can be answered with a similar experiment as described in chapter 3. Persistent low-frequency uncaging at excitatory synapses can lead to shrinkage or even removal of the dendritic spine (Oh et al., 20I3; Stein et al., 20I5). We could employ this uncaging method close to an axon-dendrite crossing with a bouton and study the response of the inhibitory bouton on this local reduction in excitation.

Another interesting question is if changes in inhibitory synapse strength or activity also affects excitatory synapses nearby. As currently there is no local method to exclusively induce inhibitory synaptic plasticity, we would need other approaches to answer this question. For example, optogenetic tools (Fenno et al., 20l I) could be used to increase or decrease the activity of inhibitory presynapses. Excitation might respond by weakening or removing synapses, which could be quantified by looking at spine morphology or uncaging EPSC amplitudes. These experiments will contribute to a better understanding of how tightly excitation and inhibition are coordinated locally.

\section{E/I coordination and disease}

E/l coordination is thought to be essential for proper dendritic integration and general neuronal functioning and disturbances of this coordination are thought to play a role in many neurological disorders. Perhaps the most obvious disorder is epilepsy, where aberrant neuronal activity is thought to be caused by hyperexcitability due to a mismatch in excitation and inhibition (Fritschy, 2008; Shao et al., 2019; Staley, 2015). Interestingly, autism spectrum disorders show a remarkable comorbidity with epilepsy (Bozzi et al., 2018). E/l imbalance due to an increase of excitation or a decrease of inhibition has been studied as a potential mechanism underlying autism (Lee et al., 20I7; Nelson and Valakh, 20I5), although a recent study suggested that an increase in $E / l$ ratio in autism is not causative but compensates for a change in cortical spiking activity (Antoine et al., 2019). A common hypothesis in schizophrenia research is that the loss of NMDA receptors on interneurons leads to a reduction in inhibition, disrupting the coordination with excitation (Kehrer et al., 2008; Tatti et al., 2017). Finally, epileptic seizures occur in some Alzheimer's disease (AD) patients and mouse models, suggesting a role for $E / l$ coordination in early stages of $A D$ as well (Busche et al., 20 I2; Palop and Mucke, 2009; Vossel et al., 2017).

For many of these disorders it is not yet known how excitation and inhibition are (mis) regulated on a dendritic and synaptic level and how this contributes to the development of the disorder. This could be explored using the experiments and findings of this thesis, in combination with mouse models of these disorders. Interestingly, endocannabinoid signaling and the use and abuse of cannabis (which contains THC, a cannabinoid) has been implicated 
in schizophrenia, epilepsy and AD (Fernández-Ruiz et al., 20I5; Katona, 20I5; Rubino et al., $20 I 5)$, suggesting that the mechanisms discussed in this thesis can be very significant for understanding these disorders.

\section{What is the impact of a single inhibitory synapse?}

Throughout this thesis, we studied subtle changes of inhibitory connectivity. Especially in chapter 3 we induce the growth of a single inhibitory bouton, a putative inhibitory synapsein-the-making. What is the physiological relevance of adding a single inhibitory synapse? While the difference might seem minimal, a single inhibitory synapse can have a very significant impact on dendritic integration. As discussed in chapter I, a single inhibitory synapse can locally affect the induction of dendritic nonlinearities such as dendritic spikes (Boivin and Nedivi, 2018; Doron et al., 2017; Lovett-Barron et al., 20I2; Müller et al., 2012). The new inhibitory synapse that could be triggered during our experiments can therefore locally interfere with supralinear summation of the excitatory synaptic inputs close by. As this inhibitory synapse is formed close to a strong and active excitatory synaptic cluster, there is a high likelihood that nonlinearities will occur in this dendritic segment. Making a new inhibitory synapse at this location could therefore be essential in preventing this synaptic cluster from having a continuous dominant effect on the somatic membrane potential and action potential generation.

The new inhibitory synapse can also have a profound effect on plasticity. An inhibitory synapse can locally prevent backpropagating action potentials and NMDA receptor activation (Doron et al., 2017; Lovett-Barron et al., 2012; Müller et al., 20I2; Müllner et al., 20I5). As NMDA receptor activation is essential for excitatory synaptic plasticity, the newly formed inhibitory synapse could locally prevent strengthening of excitatory synapses. In our experiment, we potentiate excitatory synapses to trigger local inhibitory synapse formation. A new inhibitory synapse could prevent (further) potentiation of excitatory synapses nearby, thereby ensuring that the dendritic segment does not become too strong and dominates signal integration in the dendritic tree.

Occasionally, we observed inhibitory synapses that were being formed onto a dendritic spine. Such an inhibitory synapse would have a very different effect than a shaft synapse. Since the dendritic spine is electrically highly compartmentalized, this inhibitory synapse will specifically inhibit the excitatory input of this spine (Chiu et al., 20I3). Inhibitory synapses on dendritic spines occur more often in the distal dendritic tuft (Bloss et al., 2016; Villa et al., 2016), suggesting that inhibitory synapse formation on shafts or spines is not entirely random but is somehow coordinated. Future experiments could address what the mechanisms are behind this coordination.

\section{Future perspectives: technical advancements}

\section{Understanding signaling pathways}

As discussed in chapter 2, excitatory synaptic plasticity has been studied extensively on the single synapse level.To assess the physiological relevance of the molecular pathways discussed in chapter 2, it will be important to understand the differences between LTP induction protocols and how they compare to the in vivo situation. To this end, calcium influx and activation of downstream signaling proteins should be studied while systematically varying 
protocol parameters such as pulse duration, stimulus frequency, number of repetitions, and NMDA receptor activation methods (paired vs. magnesium-free protocols). As several fluorescent probes are available that report calcium concentration or activation of specific signaling proteins (Grienberger and Konnerth, 20I2; Ueda et al., 20I3; Yasuda, 20I2), these experiments are well feasible.

Measuring the spatiotemporal pattern of calcium concentration and signal protein activation during different forms of excitatory synapse activation and plasticity could also provide us with further insights on how excitatory synaptic activity interacts with other processes.
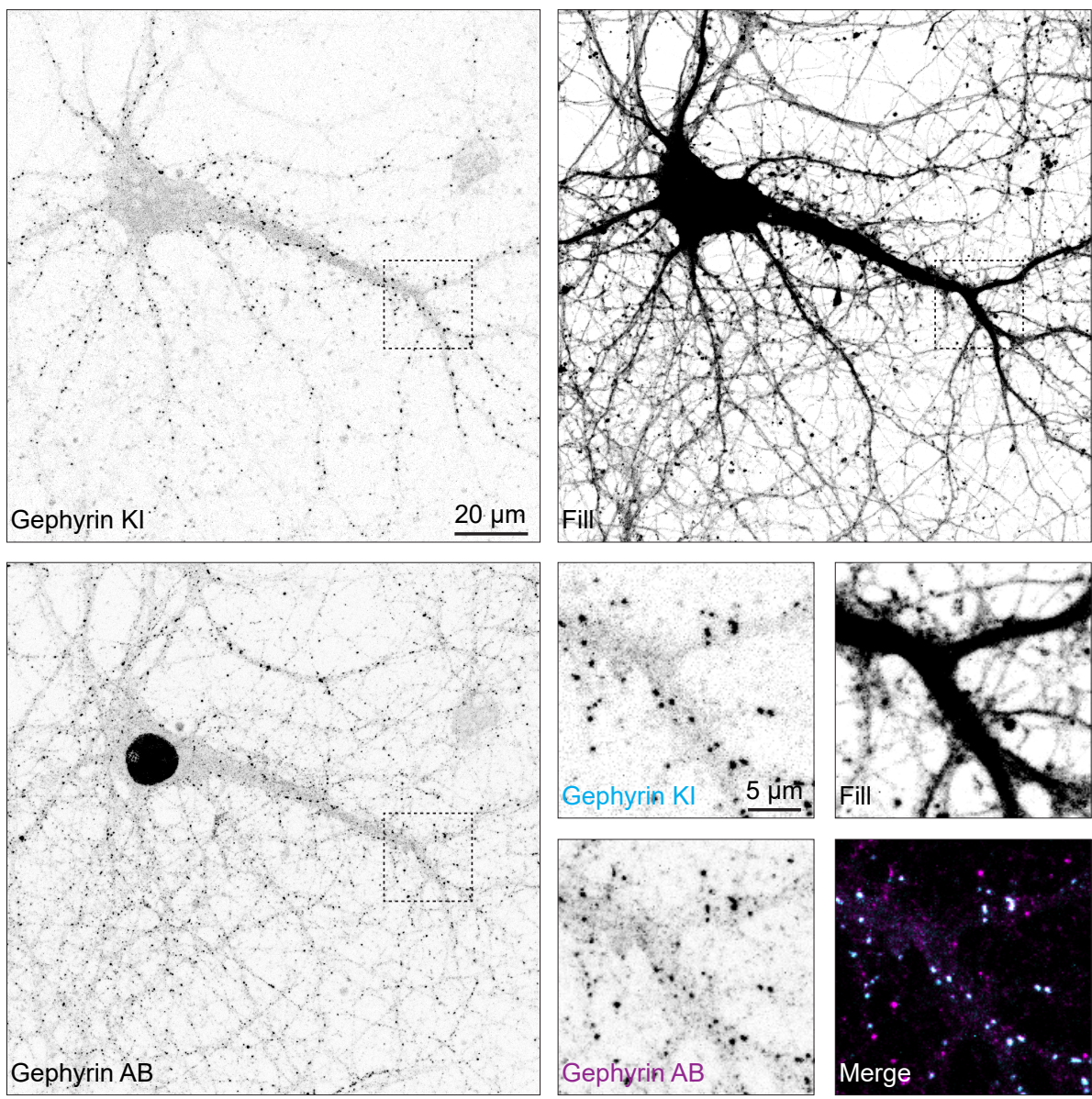

Figure 2: Knock-in of fluorescently labeled gephyrin in a dissociated hippocampal neuron

To visualize the inhibitory postsynaptic scaffolding protein gephyrin at endogenous expression levels, we are using a CRISPR-based approach. The neuron expressing the construct is labeled with dsRed-Express (top right, "fill") while endogenous gephyrin is tagged with eGFP (top left, "gephyrin Kl”). We performed immunohistochemistry with a gephyrin antibody (bottom left, "gephyrin AB") to confirm that the eGFP-labeled puncta are indeed gephyrin. We show an enlargement of the dendritic segment indicated by the dashed box (bottom right). The individual channels, as well as a merge of gephyrin $\mathrm{KI}$ (cyan) and gephyrin AB (magenta) are displayed. The merge shows that all gephyrin $\mathrm{KI}$ puncta are detected by the gephyrin $\mathrm{AB}$, confirming that all puncta represent actual gephyrin clusters. Lentiviral transduction of dissociated hippocampal neurons of rats with two constructs containing the necessary components for CRISPR-based knock-in was performed at DIV4 and neurons were fixed at DIVI4. Courtesy of René van Dorland. 
Regarding the mechanisms discussed in this thesis, reporters of the activation of CaMKII, PLC, and DAGL in the spines and dendrite would be very useful in understanding the mechanisms, timing and spread of endocannabinoid production. On the presynaptic side, a reporter of $\mathrm{CBI}$ receptor activation would help us to assess how local endocannabinoid signaling is and on what time scales the receptor is activated. Reporters of calcium concentration, filamentous actin, kinase activity and the activity of actin-modulating proteins (such as small GTPases) have been used extensively to study excitatory synaptic plasticity in the dendrite (see chapter 2), but would be helpful to study the changes in the presynaptic axon that lead to bouton formation as well.

Besides being able to observe the activation of signaling proteins, it would be useful to also be able to manipulate them. While this is often done with pharmacological compounds, this hardly provides any spatial and temporal specificity. As the local aspects of E/I coordination seem to be so important, it would be good to look for technical advancements that will allow us to manipulate signaling pathways locally. Optogenetic tools are promising for this purpose, as the genetic expression in combination with the activation by light provides both cellular and temporal specificity. Optogenetic tools are entering the subcellular regime now as well, as their efficiency is being improved and they can be targeted to specific subcellular compartments. Furthermore, there is an expanding set of tools available, such as photoactivatable ion channels, GPCRs and other signaling proteins (Rost et al., 2017). An interesting example is the use of a photoactivatable Rac that is targeted specifically to recently potentiated spines and that can make these spines shrink and depotentiate upon photoactivation (Hayashi-Takagi et al., 20I5). Using these tools in the context of inhibitory synapse formation and plasticity will likely provide interesting new insights.

\section{Assessing the functionality of inhibitory synapses}

A continuous challenge in studying inhibitory synapses is to assess their functionality. Do newly formed boutons represent functional synapses? Does bouton size correlate with synapse strength, and does a growing bouton reflect potentiation? For inhibitory boutons in general, work from our lab shows that $\sim 80-90 \%$ of inhibitory boutons colocalize with pre- and postsynaptic inhibitory synapse markers, suggesting functionality (Frias et al., 2019; Wierenga et al., 2008). For newly formed boutons, this fraction is lower but substantial, suggesting that new boutons have the potential to mature and acquire functionality (see chapter 3). Furthermore, we observed that 24 hours after repeated brief endocannabinoid treatments an increase in mIPSC frequency occurred, which is in agreement with an increase in inhibitory synapse number (chapter 4). These experiments however do not enable us to measure functionality at the level of single synapses.

To tackle this issue, we are currently exploring several approaches.As there is no morphological correlate of the inhibitory postsynapse, it would be very useful to visualize the postsynapse during live imaging using fluorescently labeled gephyrin. Several labs have visualized inhibitory postsynapses using overexpression constructs (Flores et al., 20I5; Pennacchietti et al., 2017; Villa et al.,2016).To prevent overexpression artifacts (which typically manifest as exceptionally large gephyrin puncta), we are currently following a CRISPR-cas9 based knock-in approach combined with viral transduction (Willems et al., 2019). We have managed to get good gephyrin labeling in dissociated hippocampal cultures (Figure 2) and are currently optimizing expression in hippocampal slice cultures. Fluorescently labelled gephyrin as a marker of an inhibitory postsynapse will be a valuable addition to our toolkit. It will allow us to assess changes in size and dynamics of the postsynapse, in similar ways as we have done in this 
A

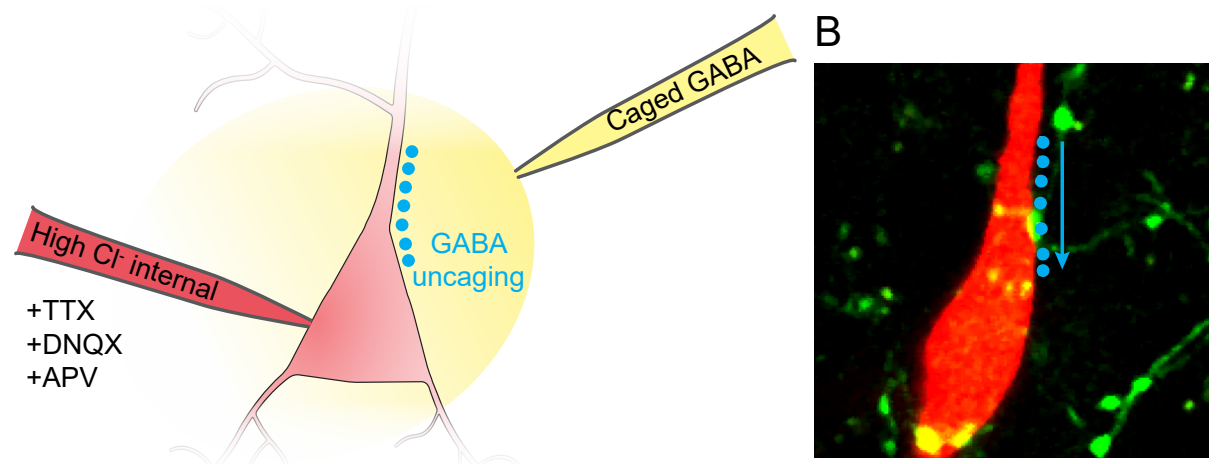

C

$20 \mathrm{pA}$

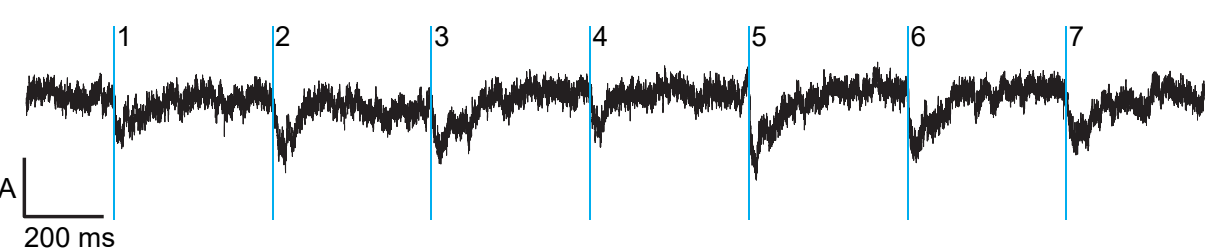

D

$\mathrm{pA} L \frac{\mathrm{s}}{1 \mathrm{~s}}$

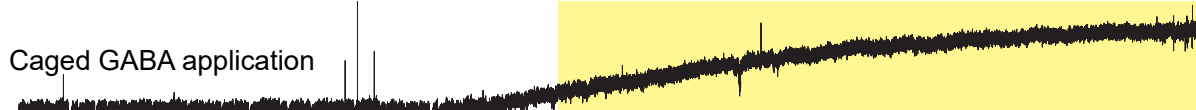

$E$

Before puff

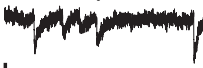

$40 \mathrm{pA}$

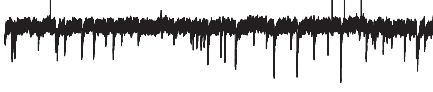

\section{$200 \mathrm{~ms}$}

Figure 3:Two-photon GABA uncaging on the soma and the primary apical dendrite

A: Patch clamp electrophysiology is performed on a CAI pyramidal neuron using an internal solution with high (70 $\mathrm{mM}$ ) chloride concentration (red) and in presence of TTX, DNQX and APV. This blocks spontaneous activity and isolates and enhances inhibitory currents. Caged GABA (yellow; courtesy of Femtonics Ltd.) is locally applied using a puff pipette. $4 \mathrm{~ms}$ uncaging pulses are performed sequentially at seven locations (blue) along the primary apical dendrite and soma.

B: Two-photon image (single plane, median filtered) of the soma and apical dendrite (red). Some inhibitory axons and boutons (green) can be seen on and around the soma. The blue dots indicate the uncaging spots and the arrow indicates the direction of the uncaging sequence.

C: Uncaging-induced inhibitory postsynaptic currents at seven locations. The blue lines indicate the moment of GABA uncaging. The numbers correspond with the order of uncaging as indicated in B.

D: Applying caged GABA (yellow, without uncaging it) leads to a strong increase of the holding current. This is in agreement with an antagonistic effect of the caged compound on GABA receptors: it suggests that caged GABA application interferes with tonic GABAergic signaling, which is excitatory in the presence of a high chloride internal solution.

E: Miniature inhibitory postsynaptic currents (mIPSCs) before (left) and during (right) local application of caged GABA. The presence of spontaneous vesicle fusion and subsequent $\mathrm{GABA}_{A}$ receptor activation leads to frequent mIPSCs in absence of caged GABA. Applying caged GABA completely abolishes mIPSCs, in agreement with an antagonistic effect of the caged compound on GABA receptors. 
thesis to assess presynaptic bouton dynamics. This can be complementary to what we know about the dynamics of gephyrin puncta in vivo (Chen et al., 20I2;Villa et al., 20I6), as we will be able to assess the dynamics on the time scale of minutes instead of days. By monitoring presynapses and postsynapses at the same time, we will be able to study how their dynamics are correlated. To get a better understanding of the molecular sequence of events that are involved in inhibitory synapse formation, fluorescently labeling other proteins of interest (such as neuroligin-2) will be important. These experiments will enable us to elucidate the formation and maturation of individual inhibitory synapses in the highest detail.

To directly assess the functionality of an inhibitory postsynapse, two-photon GABA uncaging has been used (Kantevari et al., 20I0; Kwon et al., 20I8; Villa et al., 20I6). To apply this technique to our experiments, we have started to set up this technique in our lab. As the caged GABA compound used in the aforementioned studies is not commercially available, we tested a different compound. We showed that uncaging-evoked inhibitory postsynaptic currents (uIPSCs) can be evoked on the soma and the proximal apical dendrite of CAI pyramidal neurons (Figure 3A-C). ulPSC amplitude depended on uncaging location,

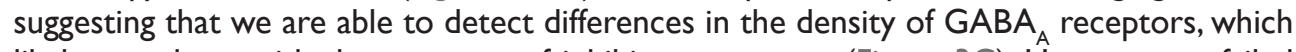
likely correlates with the presence of inhibitory synapses (Figure $3 C$ ). However, we failed to induce uIPSCs when uncaging on small dendritic branches. This seems to be caused by an antagonistic effect of the caged compound on $\mathrm{GABA}_{\mathrm{A}}$ receptors, as application of the compound without uncaging causes a significant hyperpolarization (likely a result of blocking tonic GABAergic activity while using a high internal chloride concentration) and abolishes all miniature inhibitory postsynaptic currents (Figure 3D,E).

With an improved caged GABA compound, we can directly assess postsynaptic currents and thereby measure the presence and strength of an inhibitory postsynapse over time. When combined with our uncaging-experiment of chapter 3, this would allow us to monitor if the formation of a presynaptic inhibitory bouton is followed by an increase of uIPSC amplitude on the postsynaptic dendrite. Ideally, we will be able to simultaneously monitor fluorescently labeled gephyrin puncta and assess the (changes in) synaptic strength of multiple inhibitory synapses close by. Being able to assess the functionality of individual inhibitory synapses will significantly increase our understanding of inhibitory synapse formation, plasticity, and the coordination of excitation and inhibition within a dendritic segment. Additionally, combined two-photon glutamate and GABA uncaging can be used for studying how excitatory and inhibitory synaptic currents interact, making it possible to dissect the effect of individual inhibitory synapses during dendritic integration in high detail.

\section{Concluding remarks}

The brain has always fascinated me. I find it remarkable that we are able to develop from a helpless infant into a walking and talking human, that we are capable of 'syncing' our brain with that of others and understanding their emotions, and that we have the ability to recite the lyrics of a song that we have not heard in a decade. Yet, our brains have a surprisingly hard time to comprehend how the brain might work. The brain is a complicated black box that can convert multidimensional input into a remarkably accurate response. With this thesis I have tried to have a peek inside that black box and catch a glimpse of its inner workings. By studying excitatory and inhibitory synapse formation and plasticity, and how these processes interact to coordinate excitation and inhibition, we have zoomed in on a couple of puzzle pieces of the big brain puzzle. The results presented in this thesis may contribute to an 
improved understanding of the fundamental processes that regulate and maintain neuronal function, and may therefore contribute to our understanding of neurological diseases that are a result of E/I imbalance. I am convinced that focusing on understanding these processes on the level of dendrites and synapses, especially from the perspective of the inhibitory synapse, will continue to improve our understanding of the brain. 


\section{References}

Antoine, M.W., Langberg, T., Schnepel, P., and Feldman, D. E. (20/9). Increased Excitation-Inhibition Ratio Stabilizes Synapse and Circuit Excitability in Four Autism Mouse Models. Neuron 10I, 648-66I. doi:10.1016/j.neuron.2018.12.026.

Berghuis, P., Rajnicek, A. M., Morozov, Y. M., Ross, R. A., Mulder, J., Urban, G. M., et al. (2007). Hardwiring the Brain: Endocannabinoids Shape Neuronal Connectivity. Science 316, 1212-1216. doi:I0.I I26/science. I I 37406.

Bloss, E. B., Cembrowski, M. S., Karsh, B., Colonell, J., Fetter, R. D., and Spruston, N. (20I6). Structured Dendritic Inhibition Supports Branch-Selective Integration in CAI Pyramidal Cells. Neuron 89, I016-1030. doi:I0.1016/j.neuron.2016.01.029.

Boivin, J. R., and Nedivi, E. (20/8). Functional implications of inhibitory synapse placement on signal processing in pyramidal neuron dendrites. Curr. Opin. Neurobiol. 5I, 16-22. doi:10.1016/j. conb.2018.01.013.

Bozzi, Y., Provenzano, G., and Casarosa, S. (20/8). Neurobiological bases of autism-epilepsy comorbidity: a focus on excitation/inhibition imbalance. Eur. J. Neurosci. 47, 534-548. doi:I0.1 I I I/ejn. I3595.

Busche, M. A., Chen, X., Henning, H. A., Reichwald, J., Staufenbiel, M., Sakmann, B., et al. (20I2). Critical role of soluble amyloid- $\beta$ for early hippocampal hyperactivity in a mouse model of Alzheimer's disease. Proc. Natl. Acad. Sci. 109, 8740-8745. doi:10.1073/pnas. I20617|109.

Castillo, P. E. (20I2). Presynaptic LTP and LTD of Excitatory and Inhibitory Synapses. Cold Spring Harb. Perspect. Biol. 4, I-23. doi:10.1 I0I/ cshperspect.a005728.

Castillo, P. E., Chiu, C. Q., and Carroll, R. C. (20II). Long-term plasticity at inhibitory synapses. Curr. Opin. Neurobiol. 21, 328-338. doi:10.1016/j. conb.2011.01.006.

Chen, J. L., Villa, K. L., Cha, J.W., So, P. T. C., Kubota, Y., and Nedivi, E. (20I2). Clustered Dynamics of Inhibitory Synapses and Dendritic Spines in the Adult Neocortex. Neuron 74, 36I-373. doi:I0.1016/j.neuron.20I2.02.030.

Chevaleyre,V., and Castillo, P.E. (2003). Heterosynaptic LTD of Hippocampal GABAergic Synapses: A Novel Role of Endocannabinoids in Regulating
Excitability. Neuron 38, 46I-472. doi:10.1016/ s0896-6273(03)00235-6.

Chiu, C. Q., Lur, G., Morse, T. M., Carnevale, N. T., Ellis-Davies, G. C. R., and Higley, M. J. (20/3). Compartmentalization of GABAergic Inhibition by Dendritic Spines. Science 340, 759-762. doi: I0.I I 26/science. 1234274.

Chiu, C. Q., Martenson, J. S., Yamazaki, M., Natsume, R., Sakimura, K., Tomita, S., et al. (20I8). InputSpecific NMDAR-Dependent Potentiation of Dendritic GABAergic Inhibition. Neuron 97, 368377. doi:10.1016/j.neuron.2017.12.032.

Cingolani, L.A., and Goda,Y.(2008).Actin in action:The interplay between the actin cytoskeleton and synaptic efficacy. Nat. Rev. Neurosci. 9, 344-356. doi:10.1038/nrn2373.

Cui, Y., Paillé, V., Xu, H., Genet, S., Delord, B., Fino, E., et al. (20I5). Endocannabinoids mediate bidirectional striatal spike-timing-dependent plasticity. J. Physiol. 593, 2833-2849. doi: I0.1 I I3/ JP270324.

Cui, Y., Prokin, I., Xu, H., Delord, B., Genet, S., Venance, L., et al. (20I6). Endocannabinoid dynamics gate spike-timing dependent depression and potentiation. Elife 5, I-32. doi:10.7554/ eLife. $13 \mid 85$.

Dobie, F. A., and Craig, A. M. (20II). Inhibitory Synapse Dynamics: Coordinated Presynaptic and Postsynaptic Mobility and the Major Contribution of Recycled Vesicles to New Synapse Formation. J. Neurosci. 3I, 1048I-10493. doi:10.1523/jneurosci.6023-10.2011.

Dore, K., Aow, J., and Malinow, R. (2016). The Emergence of NMDA Receptor Metabotropic Function: Insights from Imaging. Front. Synaptic Neurosci. 8, I-9. doi:I 0.3389/fnsyn.2016.00020.

Doron, M., Chindemi, G., Muller, E., Markram, H., and Segev, I. (2017). Timed Synaptic Inhibition Shapes NMDA Spikes, Influencing Local Dendritic Processing and Global I/O Properties of Cortical Neurons. Cell Rep. 21, 1550-156I. doi:10.1016/j. celrep.2017.10.035.

Fenno, L., Yizhar, O., and Deisseroth, K. (20II). The Development and Application of Optogenetics. Annu. Rev. Neurosci. 34, 389-4I2. doi:I0.1/46/ annurev-neuro-061010-1138I7.

Fernández-Ruiz, J., Romero, J., and Ramos, J.A. (20I5). "Endocannabinoids and Neurodegenerative Disorders: Parkinson's Disease, Huntington's 
Chorea, Alzheimer's Disease, and Others," in Endocannabinoids, ed. R. G. Pertwee (Springer International Publishing Switzerland), 233-259. doi:I0.1007/978-3-3|9-20825-I_8.

Flores, C. E., Nikonenko, I., Mendez, P., Fritschy, J.-M., Tyagarajan, S. K., and Muller, D. (20I5). Activitydependent inhibitory synapse remodeling through gephyrin phosphorylation.Proc. Natl.Acad. Sci. I I2, E65-72. doi: I 0. I073/pnas. I4 I I I 70 I I 2.

Frias, C. P., Liang, J., Bresser, T., Scheefhals, L., van Kesteren, M., van Dorland, R., et al. (2019). Semaphorin4D Induces Inhibitory Synapse Formation by Rapid Stabilization of Presynaptic Boutons via MET Coactivation. J. Neurosci. 39, 422 I-4237. doi:I0.1523/jneurosci.0215-19.2019.

Frias, C. P., and Wierenga, C. J. (20I3). Activitydependent adaptations in inhibitory axons. Front. Cell. Neurosci. 7, I-16. doi:10.3389/ fncel.2013.00219.

Fritschy, J.-M. (2008). Epilepsy, E/I balance and GABA receptor plasticity. Front. Mol. Neurosci. I, I-6. doi: 10.3389/neuro.02.005.2008

Ganguly, A., Tang, Y., Wang, L., Ladt, K., Loi, J., Dargent, B., et al. (20I5). A dynamic formin-dependent deep F-actin network in axons. J. Cell Biol. 210, 40 I-4 I7. doi: I0. I083/jcb.20I506 I I0.

Giuffrida, A., Parsons, L. H., Kerr, T. M., Rodríguez de Fonseca, F., Navarro, M., and Piomelli, D. (1999). Dopamine activation of endogenous cannabinoid signaling in dorsal striatum. Nat. Neurosci. 2, 358363. doi: $10.1038 / 7268$

Grienberger, C., and Konnerth, A. (20I2). Imaging Calcium in Neurons. Neuron 73, 862-885. doi:10.1016/j.neuron.2012.02.01I.

Harward, S. C., Hedrick, N. G., Hall, C. E., Parrabueno, P., Milner, T. A., Pan, E., et al. (20I6). Autocrine BDNF-TrkB signalling within a single dendritic spine. Nature 538,99-103. doi:10.1038/ naturel 9766

Hashimotodani, Y., Ohno-Shosaku, T., Tsubokawa, H., Ogata, H., Emoto, K., Maejima, T., et al. (2005) Phospholipase $C \beta$ Serves as a Coincidence Detector through Its $\mathrm{Ca}^{2+}$ Dependency for Triggering Retrograde Endocannabinoid Signal. Neuron 45, 257-268. doi:10.1016/j. neuron.2005.01.004

Hayashi-Takagi, A., Yagishita, S., Nakamura, M., Shirai, F., Wu, Y. I., Loshbaugh, A. L., et al. (20I5). Labelling and optical erasure of synaptic memory traces in the motor cortex. Nature 525, 333-338. doi: I0.1038/nature 15257.

Heifets, B. D., and Castillo, P.E. (2009). Endocannabinoid signalling and long-term synaptic plasticity. Annu Rev Pyshiol, 283-306. doi:I0.1 I46/annurev. physiol.010908.163149.Endocannabinoid.

Heifets, B. D., Chevaleyre, V., and Castillo, P. E. (2008). Interneuron activity controls endocannabinoidmediated presynaptic plasticity through calcineurin. Proc. Natl.Acad. Sci. I05, I0250-10255. doi:I0.1073/pnas.07|I880I05.

Heinbockel, T., Brager, D. H., Reich, C. G., Zhao, J., Muralidharan, S., Alger, B. E., et al. (2005). Endocannabinoid Signaling Dynamics Probed with Optical Tools. J. Neurosci. 25, 9449-9459. doi:I0.1523/JNEUROSCI.2078-05.2005.

Jung, K.-M., Astarita, G., Zhu, C., Wallace, M., Mackie, K., and Piomelli, D. (2007). A Key Role for Diacylglycerol Lipase- $\alpha$ in Metabotropic Glutamate Receptor-Dependent Endocannabinoid Mobilization. Mol. Pharmacol. 72, 6|2-62 I. doi: | 0. I | 24/mol. 107.037796

Kantevari, S., Matsuzaki, M., Kanemoto, Y., Kasai, H., and Ellis-Davies, G. C. R. (20I0). Two-color, twophoton uncaging of glutamate and GABA. Nat. Methods 7, I23-125. doi:I0.1038/nmeth.|4|3.

Katona, I. (20I5). "Cannabis and Endocannabinoid Signaling in Epilepsy," in Endocannabinoids, ed. R. G. Pertwee (Springer International Publishing Switzerland), 285-316. doi:I0.1007/978-3-31920825-I_I0.

Keck, T., Scheuss, V., Jacobsen, R. I., Wierenga, C. J., Eysel, U. T., Bonhoeffer, T., et al. (20I I). Loss of Sensory Input Causes Rapid Structural Changes of Inhibitory Neurons in Adult Mouse Visual Cortex. Neuron 7I, 869-882. doi:10.1016/j. neuron.2011.06.034

Kehrer, C., Maziashvili, N., Dugladze, T., and Gloveli, T. (2008). Altered excitatory-inhibitory balance in the NMDA-hypofunction model of schizophrenia. Front. Mol. Neurosci. I, I-7. doi: $10.3389 /$ neuro.02/006.2008

Kim, J., Isokawa, M., Ledent, C., and Alger, B. E. (2002). Activation of Muscarinic Acetylcholine Receptors Enhances the Release of Endogenous Cannabinoids in the Hippocampus. J. Neurosci. 22, 10182-10191. doi:10.1523/ JNEUROSCI.22-23-10182.2002.

Kreitzer, A. C., and Regehr,W. G. (200I). Retrograde 
Inhibition of Presynaptic Calcium Influx by Endogenous Cannabinoids at Excitatory Synapses onto Purkinje Cells. Neuron 29, 717727. doi:I0.1016/S0896-6273(0I)00246-X.

Kwon, T., Merchán-Pérez, A., Rial Verde, E. M., Rodríguez, J.-R., DeFelipe, J., and Yuste, R. (20I8). Ultrastructural, Molecular and Functional Mapping of GABAergic Synapses on Dendritic Spines and Shafts of Neocortical Pyramidal Neurons. Cereb. Cortex, I-II. doi:I0.1093/ cercor/bhyl 43.

Laguerre,A., Hauke, S., Qiu, J., Kelly, M.J., and Schultz, C. (2019). Photo-Release of 2-Arachidonoylglycerol in Live Cells. J. Am. Chem. Soc. doi:10.1021/ jacs.9b05978.

Lee, E., Lee, J., and Kim, E. (20I7). Excitation/ Inhibition Imbalance in Animal Models of Autism Spectrum Disorders. Biol. Psychiatry 8I, 838-847. doi:I0.1016/j.biopsych.2016.05.01 I.

Lovett-Barron, M., Turi, G. F., Kaifosh, P., Lee, P. H., Bolze, F., Sun, X.-H., et al. (20I2). Regulation of neuronal input transformations by tunable dendritic inhibition. Nat. Neurosci. 15, 423-430. doi: I0.1038/nn.3024.

Lu, H.-C., and Mackie, K. (20I6).An Introduction to the Endogenous Cannabinoid System. Biol. Psychiatry 79, 516-525. doi:10.1016/j.biopsych.2015.07.028.

Marsden, K. C., Beattie, J. B., Friedenthal, J., and Carroll, R. C. (2007). NMDA Receptor Activation Potentiates Inhibitory Transmission through GABA Receptor-Associated ProteinDependent Exocytosis of $\mathrm{GABA}_{\mathrm{A}}$ Receptors. J. Neurosci. 27, I4326-14337. doi:10.1523/ JNEUROSCI.4433-07.2007.

Marsden, K. C., Shemesh, A., Bayer, K. U., and Carroll, R. C. (20I0). Selective translocation of $\mathrm{Ca}^{2+} /$ calmodulin protein kinase Ila (CaMKIl $\alpha)$ to inhibitory synapses. Proc. Natl. Acad. Sci. I07, 20559-20564. doi:I0.1073/pnas. I0I0346I 07.

Matsuzaki, M., Honkura, N., Ellis-Davies, G. C. R., and Kasai, H. (2004). Structural basis of long-term potentiation in single dendritic spines. Nature 429, 76I-766. doi:10.1038/nature02617.

Müller, C., Beck, H., Coulter, D., and Remy, S. (20I2). Inhibitory Control of Linear and Supralinear Dendritic Excitation in CAI Pyramidal Neurons. Neuron 75, 85I-864. doi:10.1016/j. neuron.2012.06.025.

Müllner, F. E.,Wierenga, C. J., and Bonhoeffer,T. (20I5).
Precision of Inhibition: Dendritic Inhibition by Individual GABAergic Synapses on Hippocampal Pyramidal Cells Is Confined in Space and Time. Neuron 87, 576-589. doi:10.1016/j. neuron.2015.07.003.

Murataeva, N., Straiker, A., and MacKie, K. (20l4). Parsing the players: 2-arachidonoylglycerol synthesis and degradation in the CNS. Br. J. Pharmacol. I7I, |379-139|. doi:I0.1III/ bph.124II,

Nabavi, S., Kessels, H. W., Alfonso, S., Aow, J., Fox, R., and Malinow, R. (20I3). Metabotropic NMDA receptor function is required for NMDA receptor-dependent long-term depression. Proc. Natl. Acad. Sci. I I0, 4027-4032. doi:10.1073/ pnas. I 219454110.

Navarrete, M., and Araque,A. (2008). Endocannabinoids Mediate Neuron-Astrocyte Communication. Neuron 57, 883-893. doi:10.1016/j. neuron.2008.01.029.

Navarrete, M., Díez, A., and Araque, A. (20l4). Astrocytes in endocannabinoid signalling. Philos. Trans. R. Soc. B 369, I-7. doi:10.1098/ rstb.2013.0599.

Nelson, S. B., and Valakh, V. (20I5). Excitatory/ Inhibitory Balance and Circuit Homeostasis in Autism Spectrum Disorders. Neuron 87, 684698. doi:10.1016/j.neuron.2015.07.033.

Oh, W. C., Hill, T. C., and Zito, K. (20I3). Synapsespecific and size-dependent mechanisms of spine structural plasticity accompanying synaptic weakening. Proc. Natl. Acad. Sci. IIO, E305-E3 I2. doi:I0.1073/pnas. I2I4705 I I0.

Palop, J. J., and Mucke, L. (2009). Epilepsy and Cognitive Impairments in Alzheimer Disease. Arch. Neurol. 66, 435-440. doi:10.1001/archneurol.2009.15.

Papandréou, M. J., and Leterrier, C. (2018). The functional architecture of axonal actin. Mol. Cell. Neurosci. 91, 15I-159. doi:10.1016/j. mcn.2018.05.003.

Pennacchietti, F., Vascon, S., Nieus, T., Rosillo, C., Das, S., Tyagarajan,S.K., et al.(2017). Nanoscale Molecular Reorganization of the Inhibitory Postsynaptic Density is a Determinant of GABAergic Synaptic Potentiation. J. Neurosci. 37, I747-I756. doi:I0.1523/JNEUROSCI.05|4-I6.20I6.

Petrini,E.M., Ravasenga,T.,Hausrat,T.J., lurilli, G., Olcese, U., Racine, V., et al. (20I4). Synaptic recruitment of gephyrin regulates surface $\mathrm{GABA}_{\mathrm{A}}$ receptor 
dynamics for the expression of inhibitory LTP. Nat. Commun. 5, I-19. doi:I0.1038/ncomms492I.

Reiner,A., and Levitz, J. (20I8). Glutamatergic Signaling in the Central Nervous System: lonotropic and Metabotropic Receptors in Concert. Neuron 98, 1080-1098. doi:10.1016/j.neuron.2018.05.018.

Roland, A. B., Ricobaraza, A., Carrel, D., Jordan, B. M., Rico, F., Simon, A., et al. (20/4). Cannabinoidinduced actomyosin contractility shapes neuronal morphology and growth. Elife 3, I-23. doi:I0.7554/elife.03I59.

Rost, B. R., Schneider-Warme, F., Schmitz, D., and Hegemann, P. (2017). Optogenetic Tools for Subcellular Applications in Neuroscience. Neuron 96, 572-603. doi:10.1016/j.neuron.2017.09.047.

Roth, B. L. (2016). DREADDs for Neuroscientists. Neuron 89, 683-694. doi:10.1016/j. neuron.2016.01.040.

Rubino, T., Zamberletti, E., and Parolaro, D. (20I5). "Endocannabinoids and Mental Disorders," in Endocannabinoids, ed. R. G. Pertwee (Springer International Publishing Switzerland), 26I-283. doi:I0.1007/978-3-319-20825-I_9.

Scheefhals, N., and MacGillavry, H.D. (20I8). Functional organization of postsynaptic glutamate receptors. Mol. Cell. Neurosci. 91, 82-94. doi:10.1016/j. mcn.20I8.05.002.

Schuemann, A., Klawiter, A., Bonhoeffer, T., and Wierenga, C. J. (20I3). Structural plasticity of GABAergic axons is regulated by network activity and $\mathrm{GABA}_{\mathrm{A}}$ receptor activation. Front. Neural Circuits 7, I I3. doi:10.3389/fncir.2013.00II3.

Shao, L.-R., Habela, C.W., and Stafstrom, C. E. (2019). Pediatric Epilepsy Mechanisms: Expanding the Paradigm of Excitation/Inhibition Imbalance. Children 6, 23. doi: 10.3390/children6020023.

Shonesy, B. C., Winder, D. D. G., Patel, S., and Colbran, R. R. J. (20I5). The initiation of synaptic 2-AG mobilization requires both an increased supply of diacylglycerol precursor and increased postsynaptic calcium. Neuropharmacology 91, 57-62. doi:10.1016/j.neuropharm.2014.11.026.

Spangler, S. M., and Bruchas, M. R. (20I7). Optogenetic approaches for dissecting neuromodulation and GPCR signaling in neural circuits. Curr. Opin. Pharmacol. 32, 56-70. doi:10.1016/j. coph.2016.11.001.

Staley, K. (2015). Molecular mechanisms of epilepsy.
Nat. Neurosci. 18, 367-372. doi:I0.1038/nn.3947.

Stein, I. S., Gray, J. A., and Zito, K. (2015). Nonlonotropic NMDA Receptor Signaling Drives Activity-Induced Dendritic Spine Shrinkage. J. Neurosci. 35, 12303-12308. doi:10.1523/ JNEUROSCI.4289-I4.20I5.

Stella, N., Schweitzer, P., and Plomelli, D. (1997). A second endogenous cannabinoid that modulates long-term potentiation. Nature 388, 773-778. doi: I0.1038/420I5.

Tanaka, J.-I., Horiike, Y., Matsuzaki, M., Miyazaki, T., Ellis-Davies, G. C. R., and Kasai, H. (2008). Protein Synthesis and Neurotrophin-Dependent Structural Plasticity of Single Dendritic Spines. Science 319, 1683-1687. doi:10.1126/ science. II 52864.

Tatti, R., Haley, M.S.,Swanson, O.K.,Tselha,T., and Maffei, A. (2017). Neurophysiology and Regulation of the Balance Between Excitation and Inhibition in Neocortical Circuits. Biol. Psychiatry 8I, 82 I-83I. doi:10.1016/j.biopsych.2016.09.017.

Udakis, M., Pedrosa, V., Chamberlain, S. E. L., Clopath, C., and Mellor, J. R. (20I9). Interneuron-specific plasticity at parvalbumin and somatostatin inhibitory synapses onto CAI pyramidal neurons shapes hippocampal output. bioRxiv. doi: I0.1 I0I/774562.

Ueda,Y., Kwok, S., and Hayashi,Y. (20I3).Application of FRET probes in the analysis of neuronal plasticity. Front. Neural Circuits 7, I-19. doi:10.3389/ fncir.2013.00I63.

Viaene, A. N., Petrof, I., and Sherman, S. M. (20I3). Activation requirements for metabotropic glutamate receptors. Neurosci. Lett. 54I, 67-72. doi:10.1016/j.neulet.2013.02.004.

Villa, K. L., Berry, K. P., Subramanian, J., Cha, J.W., Oh,W. C., Kwon, H.-B., et al. (20I6). Inhibitory Synapses Are Repeatedly Assembled and Removed at Persistent Sites In Vivo. Neuron 89, 756-769. doi:10.1016/j.neuron.2016.01.010.

Vossel, K. A., Tartaglia, M. C., Nygaard, H. B., Zeman, A. Z., and Miller, B. L. (2017). Epileptic activity in Alzheimer's disease: causes and clinical relevance. Lancet Neurol. 16, 31 I-322. doi:I0.1016/SI4744422(I 7)30044-3.

Wang, W., Trieu, B. H., Palmer, L. C., Jia, Y., Pham, D. T., Jung, K.-M., et al. (2016).A Primary Cortical Input to Hippocampus Expresses a Pathway-Specific and Endocannabinoid-Dependent Form of Long- 
Term Potentiation. eNeuro 3, I-I7. doi:10.1523/ ENEURO.0160-16.2016.

Wiegert, J. S., Mahn, M., Prigge, M., Printz, Y., and Yizhar, O. (20I7). Silencing Neurons: Tools, Applications, and Experimental Constraints. Neuron 95, 504529. doi:10.1016/j.neuron.2017.06.050.

Wierenga, C. J., Becker, N., and Bonhoeffer, T. (2008). GABAergic synapses are formed without the involvement of dendritic protrusions. Nat. Neurosci. II, 1044-I052. doi:I0.1038/nn.2180.

Willems, J., de Jong, A. P. H., Scheefhals, N., and MacGillavry, H. D. (2019). ORANGE: A CRISPR/ Cas9-based genome editing toolbox for epitope tagging of endogenous proteins in neurons. bioRxiv. doi:10.1 I01/700187.

Xu, J.-Y., Zhang, J., and Chen, C. (20I2). Longlasting potentiation of hippocampal synaptic transmission by direct cortical input is mediated via endocannabinoids. J. Physiol. 590, 2305-23I5. doi: I0.1 I I3/jphysiol.20 I I.2235 II.

Yasuda, R. (20/2). Studying Signal Transduction in Single Dendritic Spines. Cold Spring Harb. Perspect. Biol. 4, I-I5. doi: I0.I I0I/cshperspect.a0056II. 


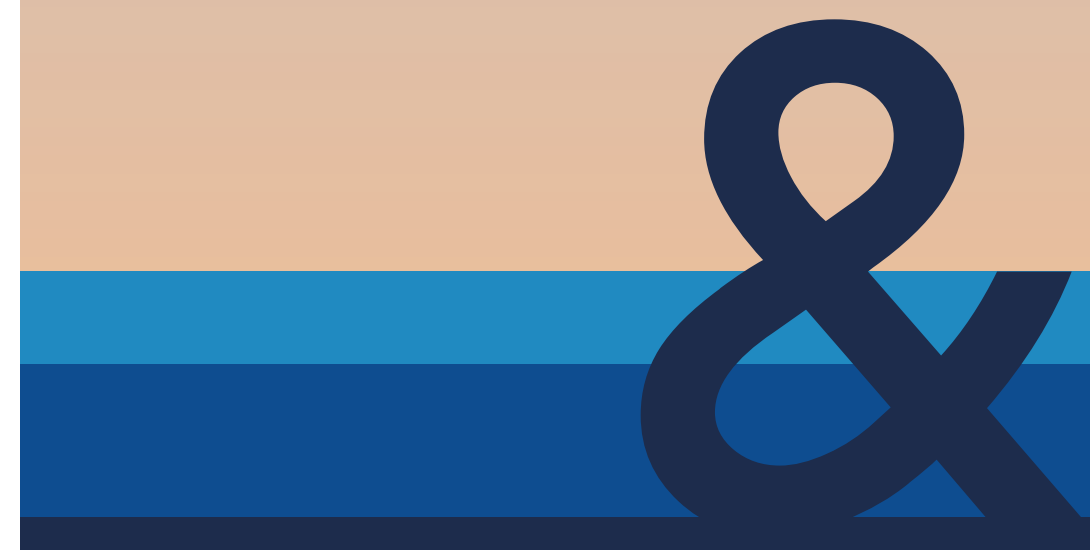




\section{Addendum}

Curriculum Vitae

List of publications

Lekensamenvatting

Lay summary

Acknowledgments 


\section{Curriculum Vitae}

Dennis Leonardus Hendrik Kruijssen was born on Saturday the I $4^{\text {th }}$ of July 1990 in Amsterdam, the Netherlands. He went to high school at the Calandlyceum in Amsterdam, where he received his Gymnasium diploma in 2008. Because of his broad interest in mathematics, physics, chemistry and biology, he chose to study Medische Natuurwetenschappen at the Vrije Universiteit. Here, he learnt to apply fundamental exact sciences to solving biological and medical problems. After a short internship in the lab of Erwin Peterman and Gijs Wuite where he worked with optical tweezers, he received his Bachelor's degree cum laude. He continued his studies at the Vrije Universiteit with a Master's in Medical Natural Sciences, with a focus on biophysics. Dennis was a member of the educational committee of Medical Natural Sciences, responsible for assessing and updating the curriculum. In his first Master's internship he returned to the lab of Erwin Peterman to perform single molecule imaging experiments on kinesin motor proteins. To broaden his horizon, he then left for London to perform an internship in the field of neuroscience in the lab of Matthew Grubb at King's College, where he studied plasticity of the axon initial segment. He returned to the Netherlands by the end of 2013, having completed his Master's degree cum laude. During his search for a suitable $\mathrm{PhD}$ position, he worked as a tutor teaching high school students in the exact sciences and discovered his passion for escape rooms working as a Game Master at Escape World Amsterdam. In 20 15, Dennis found a PhD position in a lab that aligned with his interests in neuroscience and started as a PhD candidate in the lab of Corette Wierenga. The results of his research are presented in this thesis. 


\section{List of publications}

Single synapse LTP: a matter of context?

Dennis L. H. Kruijssen and Corette J.Wierenga. Front. Cell. Neurosci. I3:496 (2019).

Endocannabinoid signaling mediates local dendritic coordination between excitatory and inhibitory synapses.

Hai Yin Hu*, Dennis L. H. Kruijssen*, Cátia P. Frias, Balázs Rózsa, Casper C. Hoogenraad and Corette J.Wierenga. Cell Reports 27, 666-675 (2019).

Rapid modulation of axon initial segment length influences repetitive spike firing. Mark D. Evans*,Adna S. Dumitrescu*, Dennis L. H. Kruijssen, Samuel E. Taylor and Matthew S. Grubb. Cell Reports 13, I233-I 245 (2015).

Single-molecule spectroscopy of motor proteins.

Bram Prevo, Seyda Açar, Dennis L. H. Kruijssen and Erwin J. G. Peterman. Encyclopedia of Analytical Chemistry (20I4).

*These authors contributed equally 


\section{Lekensamenvatting}

Ik heb het brein altijd al fascinerend gevonden. Het is bijna niet voor te stellen hoe het mogelijk is dat we ons ontwikkelen van een afhankelijke baby tot een functionerend mens; dat we ons brein kunnen "synchroniseren" met het brein van anderen en daardoor hun emoties kunnen begrijpen; en dat we in staat zijn om mee te zingen met een lied dat we al tien jaar niet gehoord hebben. Maar wat misschien wel de meest bijzondere eigenschap van ons brein is, is dat het zeer flexibel is: het past zich continu aan aan de signalen die het verwerkt. Dit stelt ons in staat om kennis te vergaren, te leren en te begrijpen. Toch vindt ons brein het verrassend lastig om te begrijpen hoe het brein werkt!

Een benadering om de mysteriën van het brein te ontrafelen, is door de bouwstenen van het brein te bestuderen. Het brein is opgebouwd uit hersencellen, ofwel neuronen. Het brein van een mens bestaat uit ongeveer 100 miljard neuronen. Dat is een I met II nullen; elk mens heeft 15 keer zo veel neuronen als het aantal mensen dat op dit moment op aarde rondloopt! Deze neuronen zijn nogal aparte cellen: ze hebben extreem lange uitlopers die ze gebruiken om contact te maken met heel veel andere neuronen. Neuronen maken gebruik van kleine elektrische stroompjes om met elkaar te communiceren. De elektriciteit loopt

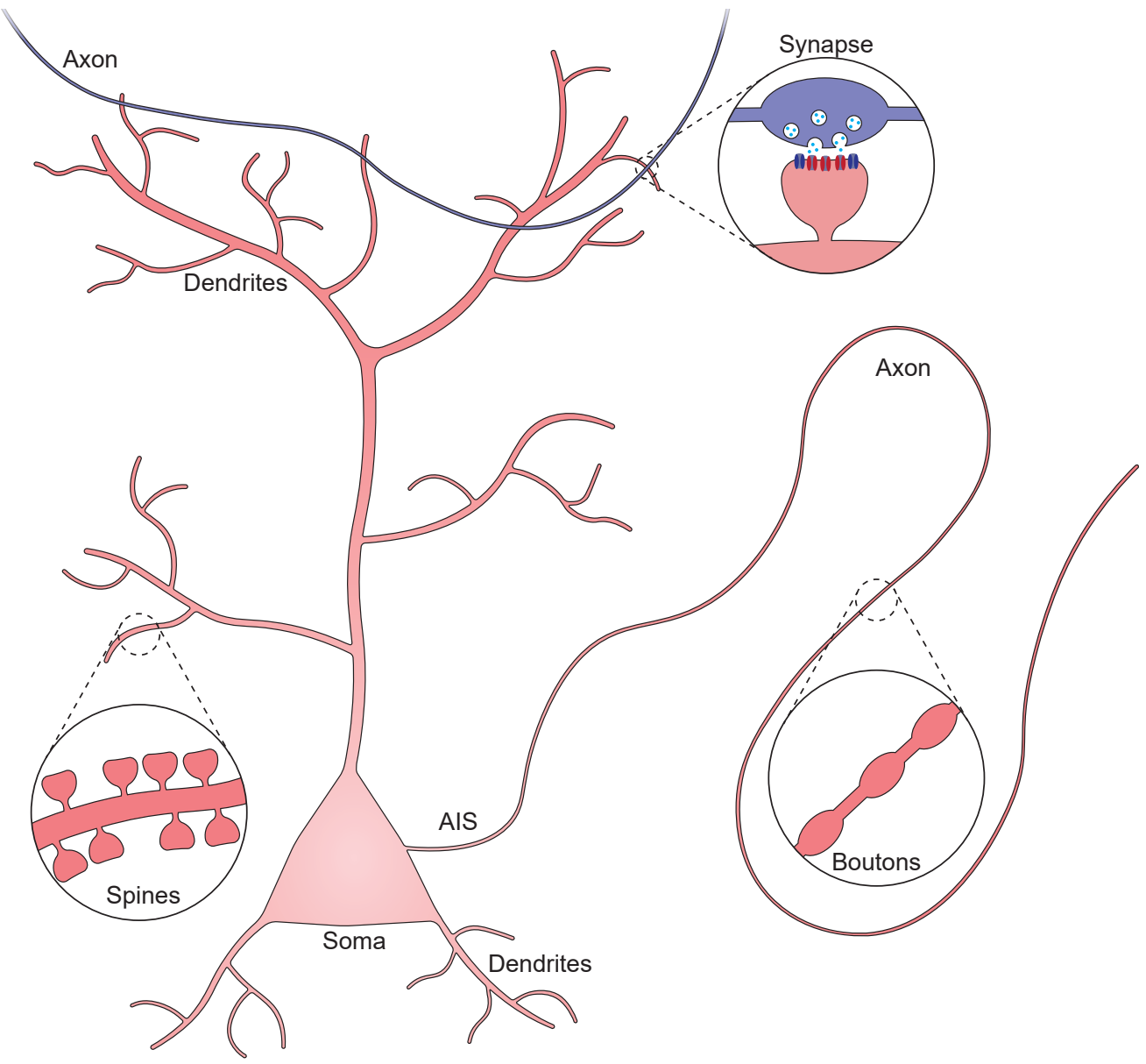


over deze uitlopers, die dus in feite als een soort elektrische kabels functioneren. Elk neuron heeft twee soorten uitlopers. Ten eerste is er het axon, welke wordt gebruikt om elektrische signalen naar de volgende neuronen te sturen. Ten tweede zijn er dendrieten. Deze zijn zeer vertakt en dienen ervoor om alle elektrische signalen op te vangen en samen te brengen. Het axon van het ene neuron maakt dus contact met een dendriet van het andere neuron. Een contactpunt tussen axon en dendriet heet een synaps. Een gemiddeld neuron maakt synapsen met wel duizenden andere neuronen!

Als we inzoomen op een synaps, zien we dat het axon daar wat verdikt is. Deze verdikking noemen we een bouton. Wanneer een elektrisch signaal het bouton bereikt, komen er signaalstoffen genaamd neurotransmitters vrij. Deze neurotransmitters zorgen ervoor dat in de dendriet van het andere neuron een nieuw signaal ontstaat. Neurotransmitters zijn er in twee "smaken." Ten eerste zijn er excitatoire ofwel stimulerende neurotransmitters. Deze zorgen voor een positief signaal in de dendriet, waardoor het waarschijnlijker wordt dat dit neuron ook weer signalen door gaat sturen. Ten tweede zijn er inhibitoire ofwel remmende neurotransmitters. Deze zorgen voor een negatief signaal in de dendriet, waardoor het juist minder waarschijnlijk wordt dat dit neuron signalen door gaat sturen. Een synaps gebruikt altijd maar één soort neurotransmitter. We spreken daarom ook wel van excitatoire en inhibitoire synapsen.

Een gemiddeld neuron heeft dus te maken met een kakafonie aan signalen: er zijn duizenden signalen die binnenkomen en verwerkt moeten worden, en vervolgens moet het neuron bepalen of het zelf een signaal naar de volgende neuronen gaat sturen. Om ervoor te zorgen dat het neuron goed blijft functioneren, is het dus belangrijk om dit proces van signaalverwerking in goede banen te leiden. Een belangrijk aspect hierbij is een goede afstemming tussen excitatoire en inhibitoire synapsen. Omdat inhibitoire synapsen een tegenovergesteld effect hebben van excitatoire synapsen, zijn ze goed in staat om excitatoire signalen te matigen of zelfs helemaal op te heffen. Zo zou je de inhibitoire synapsen kunnen voorstellen als de "verkeersregelaars" die het drukke "verkeer" (de excitatoire synapsen) in goede banen leiden. Maar dit luistert heel nauw: er moeten genoeg inhibitoire en excitatoire synapsen zijn, met de juiste sterkte en op de juiste plek in de dendriet.

De afstemming tussen excitatie en inhibitie kan op verschillende manieren gereguleerd worden. Ten eerste kan de vorming van synapsen gereguleerd worden. Synapsen worden continu gevormd en verwijderd. Dit gebeurt het vaakst in een ontwikkelend brein, maar ook in een volgroeid brein veranderen de verbindingen tussen neuronen nog steeds. Ten tweede kan de sterkte van synapsen gereguleerd worden. Synapsen kunnen sterker of zwakker worden: dezelfde synaps veroorzaakt nu een groter/kleiner signaal in de dendriet. Dit concept noemen we plasticiteit. Synapsen veranderen regelmatig van sterkte en men denkt dat dit ten grondslag ligt aan onze mogelijkheid om te leren. Als de vorming en plasticiteit van synapsen ongecontroleerd zou gebeuren, is het mogelijk dat de afstemming tussen excitatie en inhibitie verstoord wordt. Er ontstaan dan bijvoorbeeld clusters van zeer sterke excitatoire synapsen in de dendrieten, die alle signaalverwerking zullen domineren. Om dit te voorkomen zijn er waarschijnlijk allerlei mechanismen die deze processen lokaal kunnen reguleren, en dit proberen we in dit proefschrift beter te begrijpen.

Om deze lokale processen te begrijpen, is het belangrijk om eerst in kaart te brengen hoe een enkele excitatoire synaps sterker kan worden. De literatuur over dit onderwerp heb ik samengevat in hoofdstuk 2. Sinds enkele jaren is het in sommige laboratoria mogelijk om individuele synapsen te stimuleren. Deze techniek heet "two-photon glutamate uncaging": 
door middel van een laser zijn we in staat om heel lokaal, op het niveau van een enkele synaps, excitatoire neurotransmitters vrij te maken. Door middel van deze techniek beginnen we nu te begrijpen dat bepaalde stimulatiepatronen een synaps sterker kunnen maken, en krijgen we ook een steeds beter beeld van hoe dit gereguleerd wordt. Het sterker worden van een enkele synaps is zeer afhankelijk van de context: technische aspecten van het experiment, maar ook de activiteit van naburige synapsen, kunnen plasticiteit beïnvloeden. Onderzoek naar deze processen zal ons begrip vergroten van hoe naburige synapsen met elkaar samen of juist tegen elkaar kunnen werken tijdens plasticiteit.

De volgende vragen die gesteld moeten worden: hoe lokaal zijn excitatie en inhibitie op elkaar afgestemd? Gebeurt dit op het niveau van individuele synapsen? En welke mechanismen zijn hierbij betrokken? Dit hebben we onderzocht in hoofdstuk 3, waarbij we gebruik maakten van plakjes van de hersenen van muizen. Door middel van two-photon glutamate uncaging stimuleerden we een klein cluster van vier excitatoire synapsen op dezelfde dendriet. Hierdoor maakten we deze synapsen sterker, met als doel de afstemming tussen excitatie en inhibitie te verstoren. Door middel van een speciale microscopische techniek, "two-photon imaging", observeerden we dat een naburig axon een inhibitoire bouton vormde, die contact makte met de verstoorde dendriet. De vorming van een inhibitoire bouton is een van de eerste stappen in de vorming van een inhibitoire synaps. Wat hier dus waarschijnlijk gebeurt, is dat de verstoring van de afstemming in de dendriet lokaal hersteld wordt door te beginnen met het maken van een nieuwe inhibitoire synaps. We hebben vervolgens laten zien dat dit proces geregeld wordt door een groep stoffen genaamd endocannabinoiden. Deze stoffen - vergelijkbaar met de actieve component in cannabis - worden lokaal geproduceerd door de dendriet tijdens de verstoring en dit is voor het axon de trigger om een nieuw bouton te vormen.

De ontdekking dat dit lokale proces door endocannabinoiden werd gereguleerd was onverwacht omdat deze stoffen normaal gesproken vooral betrokken zijn bij het zwakker maken van bestaande inhibitoire synapsen. Wij vonden daarentegen aanwijzingen dat ze ook betrokken kunnen zijn bij de eerste stappen in de vorming van een nieuwe inhibitoire synaps. In hoofdstuk 4 werkte ik deze observatie verder uit. Ik liet zien dat wanneer ik zelf axonen lokaal blootstelde aan endocannabinoiden, dit er inderdaad voor zorgde dat deze axonen meer geneigd waren om boutons te vormen of boutons te laten groeien. Door middel van elektrofysiologie, een techniek waarmee ik alle elektrische signalen van een enkel neuron kan meten, toonde ik aan dat 24 uur na een korte behandeling met endocannabinoiden er meer inhibitoire signalen door een neuron lopen. Dit kan wijzen op een toename van het aantal inhibitoire synapsen. Wanneer ik de hersenplakjes echter te lang blootstelde aan endocannabinoiden leidde dit tot het tegenovergestelde effect.Ten slotte zijn er aanwijzingen dat endocannabinoiden ook de plasticiteit van excitatoire synapsen kunnen beïnvloeden. Samen tonen deze experimenten aan dat endocannabinoiden veel complexere effecten hebben dan bekend was. $\mathrm{Er}$ is dus nog veel onderzoek nodig om deze processen beter te begrijpen.

Samengevat gaat dit proefschrift over twee belangrijke vragen: hoe worden synaptische verbindingen in het brein gevormd? En welke regels volgen ze als ze veranderen? Ik ben ervan overtuigd dat het een essentiële stap is voor de neurowetenschappen om deze regels te ontrafelen. Als we deze regels beter begrijpen, zullen we beter begrijpen hoe de fundamentele bouwstenen van het brein hun werk doen. Hopelijk leidt dit er ook toe dat we beter begrijpen wat er misgaat in een ziek brein. Er zijn aanwijzingen dat de afstemming tussen excitatie en inhibitie verstoord is in neurologische aandoeningen zoals epilepsie, 
schizofrenie en autisme. Met dit proefschrift heb ik ingezoomd op een paar puzzelstukjes van de grote puzzel die het brein is. Ik hoop dat het werk in dit proefschrift een bijdrage levert aan het begrip van de fundamentele processen in het brein en daarmee ook het begrip van neurologische aandoeningen. Ik ben er van overtuigd dat wanneer toekomstig onderzoek zich blijft richten op het bestuderen van deze processen op het niveau van dendrieten en synapsen, met name vanuit het perspectief van de (vaak onderschatte) inhibitoire synaps, we het brein steeds beter zullen begrijpen. 


\section{Lay summary}

I have always been fascinated by the brain. It is hard to imagine how it is possible that we can develop from a dependent infant to a fully functional human; that we are able to "synchronize" our brains with the brains of others and can understand their emotions; and that we are able to sing along with a song that we have not heard in a decade. But perhaps the most amazing property of our brain is that it is highly flexible: it is continuously adapting to the signals it is processing. This allows us to gain knowledge, to learn and to understand. Despite this, our brain has a surprisingly hard time to understand how the brain works!

One approach to unraveling the mysteries of the brain is by studying its building blocks. The brain is built up out of brain cells, or neurons. The human brain consists roughly of 100 billion neurons. That is a I with II zeros; every human has 15 times more neurons than the amount of people walking the surface of the earth at this very moment! These neurons are a little quirky: the cells have extremely long processes, which they use to contact many other neurons. Neurons use small electrical currents to communicate with each other.This electricity runs over these processes, which basically function as electrical cables. Every neuron has two types of processes. Firstly it has an axon, which is used to send electrical signals to the next neurons. Secondly it has dendrites. These are highly branched and serve to collect all electrical signals and bring them together.The axon of one neuron thus contacts the dendrite of another neuron. A contact point between axon and dendrite is called a synapse. An average neuron makes synapses with thousands of other neurons!

When we zoom in on a synapse we see that there is a thickening of the axon called a bouton. When an electrical signal reaches the bouton, signaling molecules called neurotransmitters are released. These neurotransmitters trigger a new signal in the dendrite of the next neuron. Neurotransmitters come in two "flavors." Firstly, there are excitatory or stimulating neurotransmitters. These molecules trigger a positive signal in the dendrite, making it more likely that this neuron will also send out a signal. Secondly, there are inhibitory neurotransmitters. These molecules trigger a negative signal in the dendrite, making it less likely that this neuron will also send out a signal. A synapse always uses only one kind of neurotransmitter.We therefore also speak of excitatory and inhibitory synapses.

An average neuron is dealing with a cacophony of signals: there are thousands of incoming signals that need to be processed, and the neuron then needs to decide if it consequently will send a signal to the next neurons. To make sure the neuron functions properly, it is important to regulate the signal processing. An important aspect is a proper coordination of excitatory and inhibitory synapses. Because inhibitory and excitatory synapses have opposite effects, inhibitory signals are able to reduce or even cancel excitatory signals. You could imagine inhibitory synapses as the "traffic controllers" regulating the busy "traffic" (represented by the excitatory synapses). This coordination has to be tight: a neuron needs the right number and strength of excitatory and inhibitory synapses, but they also need to be in the right part of the dendrite.

The coordination of excitation and inhibition can be regulated in several ways. Firstly, synapse formation can be regulated. Synapses are continuously being formed and removed. This happens at a high frequency in the developing brain but also in a mature brain the connections between neurons are changing. Secondly, the strength of synapses can be regulated. Synapses can become stronger or weaker: the same synapse is now triggering 
a larger/smaller signal in the dendrite. This concept is called plasticity. Synapses regularly change their strengths and it is thought that this is the neuronal basis of our ability to learn. If synapse formation and plasticity would be uncoordinated, the coordination between excitation and inhibition might be disturbed. For example, clusters of very strong excitatory synapses could arise, dominating all signal processing in dendrites. To prevent this, it is likely that mechanisms are in place to locally regulate this, and it is these mechanisms that we try to understand in this thesis.

To understand these local mechanisms it is important to understand how a single excitatory synapse can be strengthened. I have summarized the literature about this topic in chapter 2. Recently it has become possible in some labs to stimulate individual synapses. This technique is called "two-photon glutamate uncaging:" using a laser, we are able to release neurotransmitters highly locally, on the level of a single synapse. Using this technique, we are now starting to understand that certain stimulation patterns can strengthen a synapse.We are also improving our understanding of how this strengthening is regulated. The strengthening of a synapse is highly dependent on context: technical aspects of the experiment, but also the activity of neighboring synapses, can affect plasticity. Studying these mechanisms will improve our understanding of how neighboring synapses can cooperate or hinder one another during plasticity.

The next questions that need to be asked: how local is the coordination between excitation and inhibition. Is it coordinated on the level of individual synapses. And what mechanisms are involved? In chapter 3, we use slices of mice brains to answer these questions. Using two-photon glutamate uncaging we stimulated a small cluster of four excitatory synapses on the same dendrite. By doing this, we strengthened these synapses and likely disturbed the local coordination between excitation and inhibition. Using a specialized microscopy technique, "two-photon imaging", we observed that a crossing axon made an inhibitory bouton contacting the disturbed dendrite. Forming an inhibitory bouton is the first step in creating a new inhibitory synapse. It is likely that disturbance of the coordination of excitation and inhibition triggers the formation of a new inhibitory synapse to restore the coordination. Next, we showed that this mechanism is regulated by a class of molecules called endocannabinoids. These molecules - which are similar to the active component of cannabis - are locally produced by the dendrite during the disturbance and this triggers the axon to start bouton formation.

The finding that this local mechanism is regulated by endocannabinoids was unexpected because this class of molecules are mostly known for being involved in weakening existing inhibitory synapses. We however found indications that endocannabinoids can also be involved in the first steps of inhibitory synapse formation. In chapter 4 I expanded on this observation. I showed that when I exposed axons to endocannabinoids locally, this indeed triggered a bias towards bouton formation and growth in these axons. Using electrophysiology, a technique that allows me to measure all electrical signals of a single neuron, I showed that 24 hours after a brief treatment with endocannabinoids more inhibitory signals were running through a neuron. This could indicate an increase in the number of inhibitory synapses. However, when I exposed the brain slices to endocannabinoids for too long, this lead to the opposite effect. Finally, there are indications that endocannabinoids also affect the plasticity of excitatory synapses. Together, these experiments show that endocannabinoids can have far more complex effects than was previously known. More research is thus needed to better understand these processes. 
This thesis is about two important questions: how are synaptic connections in the brain formed? And what rules do they follow when they are changed? I am convinced that unravelling these questions is an essential step for neuroscience. If we improve our understanding of these rules, we will better understand the workings of the fundamental building blocks of the brain. Hopefully this will also lead to an improved understanding of what goes wrong in the diseased brain. There are indications that the coordination of excitation and inhibition is disturbed in neurological disorders such as epilepsy, schizophrenia and autism. In this thesis, I zoomed in on a few puzzle pieces of the big brain puzzle. I hope the work in this thesis contributes to the understanding of fundamental processes in the brain and thereby also the understanding of neurological disorders. I am convinced that when future research will continue to focus on studying these mechanisms on the level of dendrites and synapses, especially considering the (often ignored) inhibitory synapse, we will continue to improve our understanding of the brain. 
Addendum 


\section{Acknowledgements}

Van promoveren leer je twee dingen. Ten eerste leer je hoe wetenschap in elkaar zit. Wetenschap gaat over meer dan alleen het vergaren van kennis. Het gaat over hard werken om je deadlines te halen. Het gaat over het leren kennen van interessante mensen uit alle windstreken. Het gaat over het verdedigen van je ideeën. Het gaat over het presenteren en verkopen van je verhaal.Wetenschap is traag, frustrerend en eenzaam. Maar tegelijkertijd ook elegant, fascinerend en een teamprestatie. Het tweede dat je leert van promoveren is hoe je zelf in elkaar zit. Je komt jezelf tegen tijdens een $\mathrm{PhD}$ en daardoor ken ik mezelf nu beter dan ooit. Zo heb ik het talent om alles in een nét iets te negatief licht te bekijken. Toch kijk ik met een ontzettend goed gevoel terug op mijn leven als promovendus de afgelopen 4+ jaar. Dat komt door alles wat ik over de wetenschap en over mezelf heb geleerd, maar met name door alle lieve, inspirerende en rare mensen die ik heb leren kennen, die me gesteund en er doorheen gesleept hebben. Daarom wil ik hier de ruimte nemen om iedereen te bedanken.

Allereerst wil ik mijn copromotor bedanken. Corette, wij zijn in een aantal opzichten tegenpolen. Zo ben jij al gauw optimistisch en enthousiast, terwijl ik vaak sceptisch en gereserveerd ben. Ook al gaf ik het niet graag toe, je positiviteit werkt misschien toch wel een beetje aanstekelijk. Bedankt dat je die rare, koppige jongen jaren geleden een kans hebt gegeven en bedankt voor alle keren dat je me gepusht hebt om mijn grenzen te verleggen. Daardoor heb ik de afgelopen jaren ontzettend veel geleerd. Ook dank ik mijn promotor. Casper, wanneer wij elkaar spraken was je altijd belangstellend en voelde ik me gesteund. Ook zal ik onze avonturen in Bordeaux niet snel vergeten! Ik wil ook NWO-I bedanken voor het financieren van mijn $\mathrm{PhD}$ en voor alle goede cursussen die ik dankzij jullie heb kunnen volgen. Dank aan de commissie voor het beoordelen van mijn proefschrift en verdediging: Huib Mansvelder, Helmut Kessels, Jeroen Pasterkamp, Louk Vanderschuren, Lukas Kapitein en Chantal Kemner.

Alle Pl's van onze afdeling, bedankt. Anna, ik bewonder je toewijding en je talent om de zwakke punten van ieder verhaal te vinden. Hierdoor hou je ons allemaal scherp en maak je ieder project een stukje beter. Lukas, ik herinner me nog goed de eerste keer dat we elkaar ontmoetten, toen ik - zonder dat ik dat van tevoren wist - per ongeluk ineens bij je zat te solliciteren. Wat een fiasco was dat! lk hoop dat ik de tweede keer dat we elkaar ontmoetten en in de jaren daarna een betere indruk heb gemaakt. Ik bewonder je stortvloed aan ideeën! Harold, wat heb jij ongelofelijk snel een prachtig lab uit de grond gestampt! Ik vind het ontzettend knap hoe snel je het PI-zijn onder de knie hebt gekregen en hoe goed je het nu doet. Paul, Sabrina, Ginny, Sander and Mike, thank you for your input in the weekly meetings and I wish you and your labs the best.

Lieve Phebe, moeder-overste van CellBio. Ik weet dat je het niet wil horen, maar het is waar: zonder jou dondert de hele tent in elkaar. Bedankt voor alle keren dat je me uit de brand hebt geholpen en voor alle keren dat je me aan het lachen hebt gemaakt. Wat leuk ook om nu je huisje te hebben gezien en wat kan je heerlijk koken! Ik hoop tot snel! Bart, als Phebe de moeder van de CellBio-familie is dan ben jij de lollige oom. Wat hebben we veel gelachen! Ik denk dat ik jouw specifieke droge grappen altijd "Bart-grappen" zal blijven noemen. Esther, als ik jou tegenkwam in de gang was er altijd tijd voor een gezellig praatje. Ook in mindere tijden was je altijd positief en sterk en dat bewonder ik ontzettend. llya and Eugene, thanks for everything you do to keep the department running and for every time you helped me out when I needed lens-cleaning-rockets or laser power meters. Laurens, Ron en Jan-Andries, 
bedankt en succes.

Corette heeft de neiging om mensen aan te nemen die "out-of-the-ordinary" zijn. Dat is dan ook de reden dat ik me altijd prima thuis heb gevoeld in het Wierengalab. Ik ga onze gekke wetenschappelijke familie daarom ook zeker missen. Hai Yin, wat hebben wij al die jaren fijn en intensief samengewerkt. In het begin was het een beetje onwennig, maar ik denk dat we elkaar vrij snel goed aanvoelden. Ik ben ontzettend trots op onze publicatie samen, dat hebben we toch maar mooi even geflikt! Ik waardeer je kritische houding, je aparte gevoel voor humor en je oog voor detail. Bedankt voor elke keer dat je mijn gemopper hebt aangehoord, me hebt geholpen als ik de setup weer eens had gesloopt, en elke keer dat je me geduldig aanhoorde als ik vond dat je analyseprogramma niet goed naar me luisterde. Ik zal ons gepiel aan de setups en lichtpaden, gehannes met de Hongaren en onze avontuurlijke conferentiebezoeken (kijk uit voor de tyfussteentjes!) niet snel vergeten. Geef Kaiba een dikke knuffel en ik hoop je nog vaak te zien!

Lotte, wat hebben we toch altijd heerlijk samen staan te mopperen, vloeken en tieren. Ik hou er van. Ik heb je binnen zien komen als student, hoewel je toen al behoorlijk zelfstandig was. Gelukkig maar, want ik had destijds zelf nog amper een idee wat ik aan het doen was (al hoop ik dat ik je wel nog iets heb kunnen leren). Ik weet zeker dat jij het in je hebt om een heel succesvolle wetenschapper te worden, als je er zelf maar in gelooft. En vergeet af en toe niet om alles proberen los te laten! Bedankt voor alle mooie momenten en tot snel. Carlijn, één peer één boom. Met jou kan je altijd lachen en volgens mij ben je niet chagrijnig te krijgen. Dat is echt een talent (en ik kan het weten)! Limburgse gezelligheid gecombineerd met een goed (maar soms chaotisch) stel hersens en veel doorzettingsvermogen. Daar kom je ver mee, succes! Elske, de rust zelve. In ieder geval aan de buitenkant! Wat fijn dat ik je als kantoorgenootje beter heb leren kennen. Ik heb ongelofelijk veel respect voor jouw volhardendheid. Het is jammer dat je niet hebt kunnen "shinen" in het Wierengalab, ik weet zeker dat dat onder andere omstandigheden wel gebeurd was! Ik wens je veel succes bij het RIVM, ze hebben een goede aan jou! Jian, I admire the courage it takes to travel halfway across the globe to a country with a totally different culture than where you grew up. Good luck with finishing your $\mathrm{PhD}$ ! Marvin and Ate, I wish you all the best with completing your projects, I'm sure you'll do great!

Vrienden van het MacGillavrylab. Wat hebben jullie toch een gezellig clubje mensen bij elkaar! Lieve Nicky, bedankt dat je me hebt meegesleurd naar San Diego! Dat was echt een mooi avontuur. Ik zal onze lol tijdens de SfN, in de pianobar, en op het strand niet snel vergeten. Bedankt voor alle keren dat ik aan je bureau heb mogen kletsen en klagen. Je bent een steengoede wetenschapper, vergeet dat niet! Jelmer, wijze uit het oosten. Volgens mij vergeet jij af en toe hoe goed je bezig bent! Dat CRISPR-project is super indrukwekkend en ik verwacht dat er nog meer spectaculaire proeven zullen volgen. Lisa, ik zal jou altijd herinneren met die grote koptelefoon op! Bedankt voor alle gezelligheid aan de lunchtafel! Manon, lachebekje, bedankt voor je gezelligheid en positiviteit. Anna, I love your humor, strong opinion and I admire your dedication. I wish you all the best! Arthur, dank voor de mooie verhalen en grappen aan de lunchtafel! Yolanda, it was good to meet you in Bordeaux and it is great that you chose to join Harold's lab! I think you fit in perfectly and will do great, best of luck!

Op de mensen in het Hoogenraadlab kan je altijd rekenen voor een feestje of een borrel. Feline, wat ben jij een wetenschappelijk talent! Ik weet zeker dat je met jouw analytisch vermogen, zelfstandigheid en optimisme het heel ver gaat schoppen in de wetenschap. 
Bedankt voor alle steun wanneer het met mij of mijn project even wat minder ging en voor alle gezellige praatjes op de gang. Ik waardeer het hoe oprecht geïnteresseerd je altijd bent. Bedankt ook voor alle lol die we hebben gehad bij het organiseren van de (beste) labouting! Sybren, de eeuwige antagonist. Wat hebben we toch altijd gelachen als we weer een verbaal duel hadden aan de lunchtafel! Ik ga dat echt missen. Ik weet zeker dat er ver onder die stugge buitenkant een hart van goud zit. Je bent bijna klaar, hang in there, je kan het! Robin, wat kan ik toch om jou lachen, of het nou om je chaos, je relatie met pindakaas, of je wiebelarmen gaat. Bedankt dat ik je daar al die jaren mee heb mogen plagen! Ik weet zeker dat je je project succesvol af gaat ronden, zet hem op! Robbelien, ik vind het ontzettend lief dat jij nooit te beroerd om iets te regelen of organiseren. Bedankt voor alle lol! Oh, en het verbaast me nog steeds dat we met z'n drieën in dat miniatuurautootje van je pasten! Martin, ik bewonder je onvermoeibare enthousiasme voor wetenschap en wens je veel succes in de toekomst. Eitan, you're one of the quirkiest guys I know. Thank you for being my odd but fun office mate! Lena, wat fijn dat je switch naar onderwijs gelukt is! Veel succes! Yujie, Xingxiu, Liu and Jessica, best of luck with finishing your $\mathrm{PhD}$ !

Het Kapiteinlab bestaat altijd uit de meest uiteenlopende types mensen. Wilco, wandelende encyclopedie. Wat fijn dat ik je beter heb leren kennen als kantoorgenoot. Ik had je gebrom, epistels over Amerikaanse politiek en je betogen over wetenschap niet willen missen! Ook ben ik zeer dankbaar dat je het woord "dakhaas" aan mijn vocabulaire hebt toegevoegd. Ik weet zeker dat je het in je hebt om een goede PI te worden! Klara, ik bewonder je nieuwsgierigheid. Ik vind het zo mooi hoe er onder je onschuldige buitenkant best een pittig persoon schuilt die niet bang is om een scherpe opmerking te maken! Marijn, ik vond het altijd ontzettend leuk om te zien hoe jij met je natuurkundige blik naar CellBio keek. ik waardeer het dat je nooit schroomt om een vraag te stellen! Alle succes met het afronden van je project. Chiung-Yi, Daphne and Mithila, thanks for the "gezelligheid." To the "new" people in the Kapiteinlab, good luck to you all!

Ik heb veel bewondering voor de harde werkers in het Akhmanovalab. Peter Jan, jij bent zo'n ongelofelijk aardige gast. Bedankt voor de gezelligheid en ik hoop dat de toekomst veel goeds brengt. Chao, you are the next one to defend after me. You can do it, best of luck! Babet and Boris, thanks for the good times at the lunch table! All other members of the Akhmanovalab, best of luck! Friends from Paul's lab: Sofia, thank you for your always uplifting mood! I loved our Game of Thrones-discussions. Katarina, thank you for being such a kind and caring person. I admire your strength and perseverance, I wish you all the best. To all the other members of the CellBio department, all the best!

Lieve buren van DevBio; Suzanne, Juliane and João, we had so much fun when we were office mates! Suzanne, wat fijn dat je je passie voor wetenschap hervonden hebt, veel succes! Juliane, wat hebben we een lol gehad met het organiseren van de lab outing.Veel geluk in de toekomst! João, I wish you all the best with your growing family. Vincent, bedankt voor de altijd geïnteresseerde en gezellige praatjes als we elkaar op de gang tegenkwamen. Sanne, fijn je beter te hebben leren kennen tijdens het organiseren van de lab outing. To all other members of DevBio, good luck in the future!

Marjolein, vanaf dag I tot het eind hebben wij ons gekke kantoor in Oost gedeeld. Wat hebben we daar toch altijd veel plezier gehad! Jij bent een van de meest positieve mensen die ik ken.Je bent een steengoede docent en onderzoeker! Bedankt dat ik je al die jaren altijd mocht plagen. Veel succes en tot snel! Reinier, harde werker, bedankt voor je vriendschap! Bedankt voor alle lol en alle foute grappen waar we zo hard om kunnen lachen. Succes met 
het verdedigen van je proefschrift binnenkort en we gaan elkaar nog veel zien!

Ik heb er altijd veel plezier in gehad om studenten te helpen en begeleiden. Matthijs, ik bewonder je enorm om je veerkracht - daar kom je ver mee! Na een stroeve start ging je stage daarna eigenlijk van een leien dakje en hebben we het ontzettend leuk gehad samen. Ik wens je alle succes in de toekomst. Iris, wat was jij een fijne student. Hard werken en nooit klagen (een combinatie die ik nog niet beheers!). Bedankt dat ik je altijd mocht plagen met je Volendamse afkomst. Christine, you are such a kind person! Thank you for your sincere interest and I'm sure you'll end up in a place that fits you perfectly. Aan de andere studenten van het Wierengalab, Tom, de Lisa's en Tessel, fijn jullie mee te hebben gemaakt en veel succes met jullie carrières!

Het vervelende aan de wetenschap is dat bijna iedereen een tijdelijk contract heeft en dat je dus van veel leuke mensen afscheid moet nemen. Dear Ines, you are such a sweetheart. Thank you for all the fun, kind words and good company. We had so much fun as paranymphs for Cátia, we nailed that shit! It was so good to see how you have settled in Düsseldorf with Roland and Magali, I'm sure you will be very happy there. See you soon! Lieve Anne, tijdens de eerste paar dagen van mijn $\mathrm{PhD}$, toen ik me nog verdwaasd afvroeg waar ik in godsnaam terecht was gekomen, was jij de eerste persoon die de moeite nam om een gezellig praatje met me aan te knopen. Daardoor voelde ik me meteen een beetje meer welkom en daar ben ik je eeuwig dankbaar voor. Ook bedankt voor alle lol en voor de keren dat je me even goed duidelijk maakte dat ik te veel aan het mopperen was. Ik bewonder je ongelofelijk positieve en nuchtere blik op wetenschap en ik weet zeker dat je het daar heel ver mee gaat schoppen. Roderick, ik vind het heel dapper hoe je uiteindelijk gewoon in Nederland bent gebleven en voor je postdoc compleet van onderwerp bent gewisseld.Veel succes! Marta and Daniel, thank you for all the fun moments at Eurovision evenings and other gatherings, and of course during filming for Cátia's movie. I wish you a lot of happiness with Tiago! Amélie, thank you for your good company and optimistic attitude, and I will never forget your "broodje aardappel"-plan B! Dieudonnée, jij bent echt een topper! Wat fijn dat je je plekje hebt gevonden in Maastricht! Margriet en Chris, bedankt voor alle lol bij het organiseren van de lab outing! Riccardo, Gabi, Olga, thank you for all the fun times and good chats at the lunch table. To all the other people that have left us, best of luck!

Dan wil ik graag nog wat woorden wijden aan alle mensen die me tijdens mijn opleiding hebben gesteund, geholpen en geadviseerd. Beste Iddo, bedankt voor het begeleiden van mijn Bachelorstage, het was de eerste keer dat ik in aanraking kwam met echt onderzoek en ik heb er veel van geleerd. Succes met je eigen onderzoeksgroep! Seyda, thank you for your supervision during my Master's project. Even though the project did not go as smoothly as intended, I learned a lot.Adrian, thank you for your supervision during my "extra" internship, you taught me a lot about microscopy and optogenetics! Erwin Peterman, ik zal jouw inspirerende colleges over microscopie niet snel vergeten, dat is een van de doorslaggevende factoren geweest dat ik uiteindelijk onderzoek met ingewikkelde microscopen wilde gaan doen. Bedankt voor de vele lessen tijdens mijn stages en je steun daarna, onder andere aan je aanbevelingsbrieven heb ik veel te danken. Marloes Groot, het project Zenuwwerk heeft mijn interesse in neurowetenschappen aangewakkerd, dus daar wil ik je voor bedanken. Ook het advies dat ik daarna heb mogen ontvangen, was heel fijn. Matthew Grubb, thank you for your incredible hospitality during my London internship. I had so much fun in London and I learnt a lot! I did my first practical work with neurons in your lab. This has set me on my current path and I am very grateful for that. Huib Mansvelder, bedankt voor de kans om, nadat ik was afgestudeerd, nog stage te mogen komen lopen. Het was niet het beste punt 
in mijn leven en de stage liep daardoor niet helemaal af zoals ik had gehoopt, maar ik heb in jouw lab wel elektrofysiologie geleerd! Bedankt daarvoor, want zonder die ervaring had ik nooit aan dit PhD-project kunnen beginnen. Ten slotte bedank ik alle lieve mensen bij MNW en bij MENS. Zonder alle uren in de MENS-kamer had mijn studietijd een stuk minder gezellig geweest.

Dan wordt het tijd om mijn paranimfen te bedanken. Lieve René, wat ben je toch een goede gast. Op dag I van mijn avontuur als promovendus was het ook jouw eerste dag als technicus in ons lab. Het leek me dan ook niet meer dan gepast om ook samen af te sluiten. Bedankt dat je het me vier jaar lang hebt toegestaan om je af en toe in de maling te nemen, te plagen en te kakken te zetten. En bedankt dat je ook niet schroomde om me regelmatig terug te pakken. Zonder die gein had ik de afgelopen jaren echt niet gekund.Wat fijn dat je altijd zo positief bent (dat compenseert mijn gemopper wat) en dat we altijd met elkaar konden lachen. Je hebt ook altijd hard gewerkt om ons leven als promovendi makkelijker te maken. Ik heb dat nooit vanzelfsprekend gevonden. Ik wens je een goede toekomst, veel plezier op je aanstaande bruiloft met Manon, en ik hoop dat we elkaar nog veel gaan spreken!

Dear Cátia, I consider it my greatest achievement that I managed to eventually convince you that I'm quite a nice guy. Who would have thought that after a rough start, we would turn out to be such good friends? I greatly admire (and envy) your talent as a scientist, your work ethic and your level of organization. I appreciate how you always support me, are willing to listen to me complaining, and put me in my place when needed. It is nice to know that you always have my back. Thank you for being my teacher, chocolate and cookie supplier, and life line during my PhD. Thank you for the amazing trip to Portugal, the teas at Rabarber, the dinners, the phone calls, the Mario Kart sessions, etc. I am sure I will continue to see you and Reinier a lot, as we have done over the past years, and l'm looking forward to that already.

Dan mijn lieve vrienden van buiten de UU. Zonder jullie waren mijn afgelopen jaren als wetenschapper heel saai en eenzaam geweest. Bedankt dat jullie me altijd met beide benen op de grond houden, bedankt voor de harde humor waar ik zo van hou en bedankt voor alle gezelligheid. Lieve Eetclub, wat fijn dat we na al die jaren nog steeds contact met elkaar hebben.Anke, wat fijn dat ik altijd bij je terecht kan! lk heb toch regelmatig bij je aan moeten kloppen als ik een psycholoog of job-coach nodig had. Dan ben je altijd bereid om mijn gezeik aan te horen, advies te geven en waar nodig een kritische noot te plaatsen. Ik wens je veel geluk in je nieuwe Limburgse huis met Robbert en hoop dat we elkaar ondanks de grotere afstand heel veel blijven zien! Margriet, wat was ik vereerd dat ik getuige mocht zijn op je huwelijk! Wat een mooie dag was dat. Ik waardeer je vriendschap, je kalmte en je frisse blik enorm. Ik wens je alle geluk met Eelco en we blijven elkaar spreken! Bram, ik waardeer je aparte gevoel voor humor en je gebrek aan terughoudendheid. Ik hoop dat we elkaar weer vaker gaan zien! Evelyn, welkom in Utrecht en veel succes met alle nieuwe stappen in je leven! Britt, wat hou ik toch van jouw nuchterheid. Veel succes met het afronden van je $\mathrm{PhD}$ en natuurlijk in je nieuwe huis samen met Rens! Mirelle, door de afstand zien we elkaar niet veel, maar uit het oog betekent niet uit het hart. Wat was het leuk om jouw verdediging in Bordeaux mee te maken! Ik wens jou en Raph alle geluk in de toekomst!

Lieve Esther, er gaan weinig dagen voorbij dat we elkaar niet even appen.Wat ontzettend fijn dat we al zo lang bevriend zijn. Je bent een ontzettend talentvolle classica en ik ben er zeker van dat je het ver gaat schoppen! Veel succes met het afronden van je PhD en wat er daarna gaat komen. Lieve Jerrold, wat fijn dat ik altijd op jou kan bouwen. Dag of nacht, ik kan je altijd bellen. Ik bewonder hoe dapper je (meermaals) je hele leven en carrière hebt omgegooid om 
je dromen na te jagen. Bedankt voor alle gezelligheid en humor, de mini-roadtrips en dat je me af en toe toch nog wat probeert bij te brengen over muziek.

Lieve Dian, we go way back en onze vriendschap is niet stuk te krijgen. We hebben al zo veel meegemaakt samen en daardoor weten we precies wat we aan elkaar hebben. Een van de hoogtepunten was toch wel je in-house surprise bruiloft waar ik een rol in mocht spelen. Bedankt voor alle lol op reisjes, in escape rooms, met bordspelletjes, of als we weer eens helemaal stuk gaan om iets sufs. Wat fijn ook om je zo gelukkig te zien met Marco. Marco, bedankt voor je vriendschap, voor het feit dat we je altijd in de maling mogen nemen en voor alle mooie ritjes in de Moesmobiel. Lieve kleine Hugo, je had je geen betere ouders kunnen treffen! Wat heb ik zin om jou te zien opgroeien. Caitlin, wat fijn dat je altijd zo heerlijk naïef bent en geen blad voor de mond neemt. Raak dat nooit kwijt! Kevin, bedankt voor alle mooie Center Parcs trips. Aan alle andere leden van Project B, bedankt voor alle mooie tijden en dat er nog veel mogen komen!

Lieve familie, ook al begrepen jullie niet altijd waar ik nou helemaal mee bezig was, jullie steunen me altijd. Hans en Jacqueline, jullie zijn zeker als familie voor me. Bedankt voor alle goede tijden op vakanties en verjaardagen en ik wens jullie het allerbeste. Ron, bedankt dat je mijn moeder gelukkig maakt en zo goed voor haar zorgt. Je bent een goed mens! Mike en Brian, we zien elkaar niet vaak maar als we elkaar zien, is het altijd weer als vanouds.Van vroeger herinner ik me de mooie tijden op de Nintendo64, op de tuin en op de tennisbaan. Mike en Priscilla, alle geluk samen met jullie nieuwe gezinnetje. Cisca, ik denk ontzettend vaak aan je. Ik mis je harde lach en je ongelofelijke gevoel voor humor. Wat hebben we nog een lol gehad, zelfs tot het allerlaatst! Lieve Oma, jij bent een van de sterkste mensen die ik ken. Ondanks de tegenslagen van de laatste jaren - dat we Opa nu moeten missen en dat het fysiek wat minder lekker gaat - ga je altijd stug door! Met je loopjes naar de supermarkt, met je veel te ingewikkelde puzzels en met het lezen van Engelse boeken. Wat ben ik trots op jou!

Lieve Rico, ook op jou ben ik ontzettend trots. Dat kleine broertje van me heeft het goed voor elkaar! Ik vind het ontzettend mooi om te zien hoe toegewijd jij met je passie bezig bent. We verschillen veel van elkaar, maar je blijft altijd mijn kleine broertje en ik zal altijd voor je klaarstaan. Lieve Pappa, jij hebt altijd keihard gewerkt om ons te geven wat we nodig hadden. Ik ben je ontzettend dankbaar voor het feit dat ik weet dat je me altijd steunt. Welke rare keuzes ik ook maakte, ik kon altijd op jou rekenen. Bijvoorbeeld toen je mee ging naar Londen om een huis te zoeken, of toen ik weer even bij je kwam wonen aan het begin van mijn PhD. Ik hoop dat je weet dat ik ontzettend veel van je hou. Lieve Mamma. Zonder jou was ik nooit zo ver gekomen. Je hebt me altijd gestimuleerd, gesteund en ervoor gezorgd dat ik het beste uit mezelf kan halen. Ik kan altijd met jou lachen en ik kan je altijd om advies vragen, of het nu gaat om hoe ik in godsnaam een knoop aanzet of om serieuzere zaken. Dit proefschrift draag ik op aan jou. Ik hou van jou!

Dat was het dan.

Joe! 
